\title{
POLY(N-ISOPROPYLACRYLAMIDE) \\ AT THE AIR/WATER INTERFACE
}

\author{
By
}

JU ZHANG, B. Eng., M. Eng.

\author{
A Thesis \\ Submitted to the School of Graduate Studies \\ In Partial Fulfillment of the Requirements \\ For the Degree of \\ Doctor of Philosophy
}

McMAster University

으 Copyright by Ju Zhang, September 1998 
National Library of Canada

Acquisitions and Bibliographic Services

395 Wellington Street Otawa ON K1A ONA Canada
Bibliothèque nationale du Canada

Acquisitions et senvices bibliographiques

395, no Wellington

Oumwe ON KIA ONA

Cansada
The author has granted a nonexclusive licence allowing the National Library of Canada to reproduce, loan, distribute or sell copies of this thesis in microform, paper or electronic formats.

The author retains ownership of the copyright in this thesis. Neither the thesis nor substantial extracts from it may be printed or otherwise reproduced without the author's permission.
L'auteur a accordé une licence non exclusive permettant à la Bibliothèque nationale du Canada de reproduire, prêter, distribuer ou vendre des copies de cette thèse sous la forme de microfiche/film, de reproduction sur papier ou sur format électronique.

L'auteur conserve la propriété du droit d'auteur qui protège cette thèse. $\mathrm{Ni}$ la thèse ni des extraits substantiels de celle-ci ne doivent être imprimés ou autrement reproduits sans son autorisation. 


\section{POLY(N-ISOPROPYLACRYLAMIDE)}

\section{AT THE AIR/WATER INTERFACE}


DOCTOR OF PHILOSOPHY

(Chemical Engineering)
McMaster University

Hamilton, Ontario

TITLE: $\quad$ Poly $(N$-isopropylacrylamide $)$ at the Air/Water Interface

AUTHOR: Ju Zhang

M. Eng. (Chengdu University of Science and Technology, P. R. China)

B. Eng. (Chengdu University of Science and Technology, P. R. China),

SUPERVISOR: Professor Robert Pelton

NUMBER OF PAGES: 319 


\section{ABSTRACT}

Linear polymers, copolymers, microgels and macrogels based on $\mathrm{N}$ isopropylacrylamide were prepared and the properties of these materials at the air/water interface were investigated. Dynamic surface tensions were measured by pendent drop experiments in which either the drop volumes were constant or were oscillated. The ability of colloidal microgels to lower surface tension is unusual and, for the first time, Environmental Scanning Electron Microscopy provided images showing an ordered array of $500 \mathrm{~nm}$ diameter microgels adsorbed at the air/water interface.

The surface tension of poly( $\mathrm{N}$-isopropylacrylamide) homopolymers and microgels was about $43 \mathrm{~mJ} / \mathrm{m}^{2}$ at $25^{\circ} \mathrm{C}$, and surface tension decreased slightly with increasing temperature between $25-40^{\circ} \mathrm{C}$. The temperature insensitivity of surface tension is remarkable because in solution the linear polymer phase separated and the microgel were dehydrated when the temperature was raised from 25 to near $40^{\circ} \mathrm{C}$. The introduction of hydrophilic acrylamide moieties increased surface tension and the results were fit to a one-parameter model analogous to the Margules model for the free energy of mixing of solutions.

Kinetic models were developed and fitted to the dynamic surface tension data. A key challenge was relating surface tension to the amount of adsorbed 
material at the interface. Results are shown for calculations based on both published data and statistical mechanical models of adsorbed polymer. The inability of mass transport models to predict the kinetics suggested that rearrangement of gels or linear polymer at the interface contributed to the kinetics.

Macroscopic water swollen gels like the linear polymers and the microgels displayed a low surface energy due to the accumulation of isopropyl groups at the interface. However, unlike the microgels and linear polymers, the surface characteristics reflected in contact angle measurements was sensitive to temperature in the critical range 35 to $40^{\circ} \mathrm{C}$. 


\section{PREFACE}

This thesis is composed of research work carried out by me and supervised by Dr. R. Pelton during the period of January 1994 to September 1998. The main task of this thesis is to investigate the behaviors of PNIPAM polymers at the air/water interface.

Chapters 2, 3 and 4 are submitted manuscripts with Dr. R. Pelton as the co-author, who is also my supervisor. Chapter 2 have been accepted by Colloids and Surfaces, Chapter 3 submitted to Langmuir and Chapter 4 accepted by Journal of Polymer Science Part A: Polymer Chemistry. A manuscript based on Chapter 5 is in preparation. Chapter 6 has been published in Langmuir (Zhang, Ju and Pelton, R., Langmuir, 12, 2611 (1996).) with Dr. R. Pelton and Dr. Y. Deng as the co-authors. Dr. Y. Deng's contribution to Chapter 6 was to train me to prepare the gels. In addition to his countless suggestions, critical and editorial comments, Dr. Pelton also contributed to the papers in calculations and modeling, which includes all the self-consistent field calculations and the most of the langmuir kinetic model calculations in Chapters 3 and 5, and to propose the oneconstant Margules model in modeling the copolymer surface tension in Chapter 4. I designed and carried out all the experiments, made all the $N$ isopropylacrylamide polymers and processed all the first-hand experimental data. 
I did most of the calculations and all the curve fitting, proposed and developed the modified EC models in modeling the surface tension of the homopolymers in Chapter 3. I also performed the final implementations of most of the models, except the self-consistent field calculation and the Langmuir kinetic model calculation in Chapter 3.

To make this thesis a continuous body of work, slight modifications are done to the published and submitted chapters with their main contents kept intact. Written permission for reprint of the published content of Chapter 6 was obtained from the Copyright Office of American Chemical Society. Information not included in the published or submitted papers has been attached to the end of each chapter as appendices. 


\section{ACKNOWLEDGEMENT}

My special thanks must go to my supervisor, Dr. Robert Pelton, who had given me the precious opportunity to start my endeavor toward my Ph. D. degree. His expertise in colloid and surface science has provided me with irreplaceable guidance through out my work. His twelve years of experience investigating poly( $N$-isopropylacrylamide) polymers has been my continuous source of ideas. I thank him for his time and patience, his support and encouragement, which have been accompanying me through my hard time in personal life and my every step of academic success.

I would like to thank Dr. Philip E. Wood, Dr. Donald R. Woods and Dr. John L. Brash for their support and help. They are my professional role models in the respect of their integrity and teaching practices. Working with Dr. Wood and Dr. Woods as a teaching assistant has been my best TA experience for my whole Ph. D. student life. I would like to thank Dr. Cameron M. Crowe for his help with my mathematical problems. I also would like to thank many other professors in our Department of Chemical Engineering for their support and help during my stay at McMaster.

I want to extend my appreciation to three professors in the Department of Chemistry: Dr. Michael A. Brook for his constructive comments in my supervisor 
committee meetings, Dr. Harald D. H. Stöver and Dr. Françoise W. Winnik for allowing me using their equipment.

Professor A. Wilhelm Neuman and Dr. Yu Hing Daniel Kwok in the Department of Mechanical Engineering at University of Toronto have given me a great help in my dynamic surface tension measurement, through which I obtained the majority of data for this thesis. I thank them for their kindness and friendship. I must also thank Professor Masami Kawaguchi in the Department of Chemistry for Materials at Mie University in Japan. His valuable data provide the only experimental link between surface tension and PNIPAM concentration at the air/water interface, which offers me a chance to compare the adsorption models presented in Chapter 3 with experimental results. My appreciation must also be extended to Professor Terry Cosgrove at Bristol University and Professor Gerard Fleer at Wageningen Agricultural University for providing us Goliad Version 2.1.0.0 for the self-consistent field calculation, even though I never met them.

Dr. L. Kris Kostanski, a research scientist in our department, has been helping me with equipment and problem solving in experiments for all the years. I thank him for his friendship, patience and time.

I would like to extend my appreciation to Ms. Marcia West at the Life Sciences Electron Microscopy Facility. Thanks to her hardworking, we took the in situ ESEM picture of the microgels (Figure 5.2), which is the first picture of microgels actually adsorbed at the air/water interface ever seen and reported. I viii 
would also like to thank Mr. Yew Meng Heng at the Electron Microscopy Facility of Faculty of Health Sciences for preparing the TEM pictures for my microgels.

My Ph. D. work was mostly funded by the Canadian Natural Science and Engineering Research Council (NSERC). I enjoyed three terms of Ontario Graduate Scholarship (OGS). I was also selected to receive a Clifton W. Sherman Graduate Scholarship and a Shell Canada Graduate Fellowship from the School of Graduate Studies, and an I.C.I Scholarship from I.C.I. Canada, In. I sincerely thank all the parties that generously supported me financially and spiritually. Their support makes my dream come true.

My special appreciation goes to our departmental administrative coordinator, Ms. Sara Gallo-O'Toole. Her professional management has kept our activities in order. I thank her for her friendship, understanding and help to a foreign student. My memory about Chemical Engincering is not complete without our departmental secretary, Ms. Barbara Owen. Her cheerful spirit, assistance and friendship have accompanied me through the years.

During my stay at Chemical Engincering, I received countless help from our departmental mechanical technician, Mr. Paul Gatt. His forever smile encouraged me go to him for rescue one time after another. Ms. Justyna Derkach as a departmental safety supervisor has watched my every action handling the stinky monomer, $N$-isoproplyacrylamide. I admire her responsible altitude toward 
her duty and thank her for all the help she has given to me. I am also grateful to Mr. Doug Keller, our lab manager, for his patience and help.

During the four years I spent in Pelton's group, people have come and gone. I met several good scientists. One of them is my former colleague Professor Yulin Deng. He impressed me with his expertise covering polymer chemistry and physics, analytical chemistry and equipment, colloid and surface chemistry. I benefited tremendously from his precise and instructive answers to those research problems I asked him about. Dr. Ian J. McLennan is another real scientist, whose insight in nuclear magnetic resonance spectra and dynamic light scattering, whose helping hand and unique humor will be remembered forever. I also learned a great deal from Dr. Zhuo Yang in chatting about static light scattering.

My fellow graduate students have made my life in Chemical Engineering enjoyable. I thank them all for their friendship, companion and help.

My ineffable appreciation goes to my husband, Dr. HongJie Yang. He is my best friend and math tutor. I thank him for his patience and support. And finally, this thesis is dedicated to my mother and father for their endless love and support. 


\section{TABLE OF CONTENTS}

ABSTRACT

PREFACE

ACKNOWLEDGEMENT vii

TABLE OR CONTENTS Xi

LIST OF FIGURES $\quad$ xvii

LIST OF TABLES $\quad$ xxv

LIST OF SYMBOLS xxvii

LIST OF PUBLICATIONS xxxii

Chapter 1 Introduction 1

$1.1 \quad N$-isopropylacrylamide polymers 1

1.2 Concepts of surface, interface and surface tension 4

1.3 Background work on water-soluble polymers at the air/water interface $\quad 6$

1.4 Background work on contact angle on water-swollen macrogels

8

1.5 The motivation, the theme and the objectives of the thesis

1.6 The surface tension measurement 13

$\begin{array}{ll}\text { 1.7 The scope of the thesis } & 19\end{array}$

1.8 Outline of the thesis 21

$\mathbf{x i}$ 
Chapter 2 The Dynamic Behavior of Poly( $N$-isopropylacrylamide) at the Air/Water Interface

2.1 Introduction

2.2 Experimental

2.2.1 Synthesis and purification

2.2.2 Characterization

2.2.3 Dynamic light scattering

2.2.4 Surface tension measurement

$2.3 \quad$ Results

2.3.1 Dynamic light scattering

2.3.2 Static drop volume experiment

2.3.3 Oscillating drop volume experiment

2.4 Discussion

2.5 Conclusion

2.6 Acknowledgement

2.7 References

Appendix 2.1 Calculation of $R_{z}$ and $C_{0}$

Appendix 2.2 Pendent Drops and Corresponding Surface Tensions, Area and Volume 
Appendix 2.4 An Example of Fitting the Dynamic Surface Tension Data to HuaRosen Equation

Appendix 2.5 Empirical Relationship between Surface Tension and Surface Concentration

Appendix 2.6 Calculation of the Bulk Depletion

Appendix 2.7 Calculation of the Number of PNIPAM Chains per Phaseseparated Particle

Chapter 3 The Application of Polymer Adsorption Models to Dynamic Surface Tension

Abstract

96

3.1 Introduction

97

3.2 Theoretical aspects

101

3.2.1 The Eijk \& Cohen Stuart (EC) model

101

3.2.2 Relating surface concentration to surface tension $(\gamma-$ to- $\Gamma) \quad 103$

3.2.3 Modeling oscillating pendent drop (OPD) Experiments 106

3.3 Results

110

3.3.1 Modeling static pendent drop (SPD) surface tension kinetics

3.3.2 Modeling oscillating pendent drop (OPD) surface tension kinetics

3.5 Discussion

3.6 Conclusions

3.7 Acknowledgement 
$\begin{array}{llr}3.8 & \text { References } & 129\end{array}$

Appendix 3.1 Modeling the Dynamic Surface Tension 131

Appendix 3.2 Fitting the SCF Calculation Data 156

Appendix 3.3 Calculation of the Slopes of the Dynamic Surface Tension Curves of $40 \mathrm{ppm}$

158

Chapter 4 The Surface Tension of Aqueous Poly(N-isopropylacrylamideco-acrylamide) 160

$\begin{array}{ll}\text { Abstract } & 160\end{array}$

$\begin{array}{llr}4.1 & \text { Introduction } & 162\end{array}$

$\begin{array}{lll}4.2 & \text { Experimental } & 164\end{array}$

4.2.1 Preparation of the polymers 164

4.2.2 Characterizations of the copolymers 165

$\begin{array}{ll}\text { 4.2.3 Cloud points } & 166\end{array}$

4.2.4 Heats of phase separation $(\Delta \mathrm{H}) \quad 167$

4.2.5 Dynamic surface tension measurement 167

$\begin{array}{lll}4.3 & \text { Results } & 169\end{array}$

$\begin{array}{ll}\text { 4.3.1 Cloud point temperature (CPT) } & 169\end{array}$

$\begin{array}{ll}\text { 4.3.2 Heats of phase separation } & 170\end{array}$

$\begin{array}{lll}\text { 4.3.3 Dynamic surface tension } & 171\end{array}$

$\begin{array}{lll}4.4 & \text { Discussion } & 175\end{array}$

$\begin{array}{lll}4.5 & \text { Conclusion } & 181\end{array}$

$\begin{array}{lll}4.6 & \text { Acknowledgement } & 182\end{array}$ 
Appendix 4.1 Examples of ${ }^{1} H$ NMR Spectrum of Copolymers 185

Appendix 4.2 Examples of DSC traces 188

Appendix 4.3 Calculation of the Mean Sequence Lengths 192

Chapter 5 The Surface Tension Lowering Abilities of Poly(N. isopropylacrylamide) Microgels 193

Abstract 193

$\begin{array}{lll}5.1 & \text { Introduction } & 195\end{array}$

$\begin{array}{lll}5.2 & \text { Experimental } & 199\end{array}$

5.2.1 Preparation of the microgels 199

5.2.2 Microgel characterizations 202

5.2.3 Surface tension measurement 206

$\begin{array}{lll}5.3 & \text { Results } & 209\end{array}$

5.3.1 Microgel characterizations 209

5.3.2 Dynamic surface tension measurement 214

$\begin{array}{lll}5.4 & \text { Discussion } & 221\end{array}$

$\begin{array}{lll}5.5 & \text { Conclusions } & 234\end{array}$

5.6 Acknowledgement 235

$\begin{array}{lll}5.7 & \text { References } & 236\end{array}$

Appendix 5.1 Extrapolations of Eff. Diam. $\left(D_{E}\right)$ to Zero Angle and Zero Concentration 241

Appendix 5.2 The Dissymmetry Method - $R_{z} 248$

Appendix 5.3 Langmuir Kinetics for Microgels 258 
Appendix 5.4 Approximation of the Meso-equilibrium Surface Concentration of $N$-isopropyl Groups

Chapter 6 The Temperature Dependent Contact Angles of Water on Poly(N-isopropylacrylamide) Gels 266

$\begin{array}{ll}\text { Abstract } & 266\end{array}$

$\begin{array}{lll}6.1 & \text { Introduction } & 267\end{array}$

$\begin{array}{lll}6.2 & \text { Experimental } & 270\end{array}$

$\begin{array}{lll}6.3 & \text { Acknowledgement } & 272\end{array}$

$\begin{array}{lll}6.4 & \text { References } & 273\end{array}$

$\begin{array}{lll}\text { Chapter } 7 \text { Conclusion } & 274\end{array}$

7.1 Conclusion on NIPAM polymers at the air/water interface 274

$\begin{array}{lll}7.2 & \text { Contribution } & 286\end{array}$ 


\section{LIST OF FIGURES}

Figure 1.1 The chemical structures of NIPAM monomer, homopolymer, poly( $N$-isopropylacrylamide-co-acrylamide) copolymer and PNIPAM crosslinked by $N, N^{\prime}$-methylenebisacrylamide.

Figure 1. 1 Scheme of surface tension measurement using ADSA-P.

Figure 2.1 The hydrodynamic diameters of phase-separated PNIPAM colloidal aggregates at $40{ }^{\circ} \mathrm{C}$ as a function of the solution concentration before phase separation. (The error bars indicate the relative standard deviations (it is between 1.02 to $2.45 \%$ for L-6B and 1.69 to $6.66 \%$ for $(-4)$.

Figure 2. 2 The concentration dependence of the dynamic surface tension of PNIPAM (L-6B) aqueous solutions at $25^{\circ} \mathrm{C}$.

Figure 2.3 The concentration dependence of the dynamic surface tension of PNIPAM (L-6B) aqueous solutions at $40^{\circ} \mathrm{C}$.

Figure 2.4 The concentration dependence of the dynamic surface tension of PNIPAM (L-4) aqueous solutions at $25^{\circ} \mathrm{C}$.

Figure 2.5 The concentration dependence of the dynamic surface tension of PNIPAM (L-4) aqueous solutions at $40^{\circ} \mathrm{C}$.

Figure 2.6 $t^{*}$ as a function of polymer concentration, temperature and molecular weight. 
Figure 2.7 The temperature dependence of the dynamic surface tension of PNIPAM (L-6B) 10 ppm aqueous solution.

Figure 2.8 The temperature dependence of the dynamic surface tension of PNIPAM (L-4) 10 ppm water solution.

44

Figure 2. $9 t^{*}$ as a function of temperature for $10 \mathrm{ppm}$ PNIPAM L-6B and L-4.

Figure 2. 10 Meso-equilibrium surface tensions as functions of temperature.

46

Figure 2. 11 The dynamic surface tension of $10 \mathrm{ppm} \mathrm{L-6B}$ aqueous solution as a function of time at different oscillating frequency periods at 25 ${ }^{\circ} \mathrm{C}$.

Figure 2. 12 The amplitude of steady-state surface tension fluctuations as a function of the expansion factor $\alpha$ which is the maximum drop area divided by the minimum area.

Figure 2. 13 The dynamic surface tension of $50 \mathrm{ppm} \mathrm{L-6B}$ aqueous solution as a function of time with the same oscillating periods $(P=33.55 \mathrm{~s})$ but at different temperatures.

Figure 2. 14 Surface tension of aqueous PNIPAM as a function of surface concentration interpolated from Kawaguchi's data [18]. The parameters in the Scheutjens-Fleer calculations are: interaction parameters (kT/site) water/air 6.3, polymer/air 2.435, polymer/water 0.45 ; lattice depth 30 ; chain length 100; and area per site $0.3 \mathrm{~nm}^{2}$.

xviii 
Figure 3.1 Suface tension of aqueous PNIPAM as a function of surface concentration interpolated from Kawaguchi's data [27] and from self-consistent field theory. The parameters in the SCF are: interaction parameters (kT/site) water/air 6.3, polymer/air 2.435 , polymer/water 0.45; lattice depth 30; chain length 100; and area per site $0.3 \mathrm{~nm}^{2}$.

105

Figure 3.2 Surface tension versus time for poly( $N$-isopropylacrylamide). The numbers besides the curves indicate the polymer concentration in $\mathrm{mg} / \mathrm{L}$ (ppm). The solid lines were computed using the Langmuir kinetics model together with $\Gamma$-to- $\gamma$ transformation from SCF.

Figure 3.3 Surface tension versus time curves computed with the EC model in conjunction with the $\Gamma$-to- $\gamma$ from self-consistent field calculations.

112

Figure 3.4 An example of the experimental control of the pendant drop area. The circles are experimental values of the drop surface area for $\mathrm{L}$ 6B $10 \mathrm{ppm}$ solution in a oscillating period of $24.2 \mathrm{~s}$, the solid line is the curve of the corresponding mathematical description $\left(R^{2}=0.9825\right)$ :

$$
A(t)=0.3204+0.0978\left(\cos \left(\frac{2 \pi t}{24.2}-0.0918\right)\right)
$$

Figure 3.5 The $C_{b}$ dependence of $\beta(t), \Gamma(t)$ and $y(t)$ for a moving interface as predicted by the model. $k=6.0 \times 10^{-7} \mathrm{~m} / \mathrm{s}, \quad a_{0}=1.27 \mathrm{~m}^{2} / \mathrm{mg}$. $A_{0}=0.3226 \mathrm{~cm}^{2}, A_{1}=0.0818 \mathrm{~cm}^{2}, f=0.03 \mathrm{~Hz}$ (or $P=33.6 \mathrm{~s}$ ), $\phi=0.2276$ radian. 
Figure 3. 6 The lower plot shows experimental results from OPD and SPD experiments. The upper curve shows $\Delta \gamma=\gamma_{\text {madel }}-\gamma_{\text {cop }}$. Parameters were $k=6 \times 10^{-7} \mathrm{~m} / \mathrm{s} ; C_{b}=10 \mathrm{ppm} ; a_{0}=1.3482 \mathrm{~m}^{2} / \mathrm{mg} ; f=0.04 \mathrm{~Hz}$ (or $P=24.2 \mathrm{~s}) ; A_{0}=0.3204 \mathrm{~cm}^{2} ; A_{1}=0.0978 \mathrm{~cm}^{2}$ and $\phi=0.0664$ radian.

Figure 3.7 Comparison of experimental results with the model predictions. $k=6 \times 10^{-7} \mathrm{~m} / \mathrm{s} ; C_{b}=10 \mathrm{ppm} ; a_{0}=1.25 \mathrm{~m}^{2} / \mathrm{mg} ; f=0.03 \mathrm{~Hz}$ (or $P=33.6 \mathrm{~s}$ ); $A_{0}=0.3226 \mathrm{~cm}^{2} ; A_{1}=0.0818 \mathrm{~cm}^{2}$ and $\phi=0.2276$ radian.

Figure 3.8 Comparison of experimental results with the model predictions. $k=6 \times 10^{-7} \mathrm{~m} / \mathrm{s} ; C_{b}=10 \mathrm{ppm} ; a_{0}=1.24 \mathrm{~m}^{2} / \mathrm{mg} ; f=0.02 \mathrm{~Hz}$ (or $P=60.1 \mathrm{~s}$ ); $A_{0}=0.3139 \mathrm{~cm}^{2} ; A_{1}=0.0507 \mathrm{~cm}^{2}$ and $\phi=0.1076$ radian.

Figure 3.9 Comparison of experimental results with the model predictions. $k=6 \times 10^{-7} \mathrm{~m} / \mathrm{s} ; C_{b}=50 \mathrm{ppm} ; a_{0}=1.00 \mathrm{~m}^{2} / \mathrm{mg} ; f=0.03 \mathrm{~Hz}$ (or $P=33.6 \mathrm{~s}$ ); $A_{0}=0.3226 \mathrm{~cm}^{2} ; A_{1}=0.0818 \mathrm{~cm}^{2}$ and $\phi=0.2276$ radian.

Figure 3. 10 Comparison of experimental results with the model predictions. $k=6 \times 10^{-7} \mathrm{~m} / \mathrm{s} ; C_{b}=50 \mathrm{ppm} ; a_{0}=1.00 \mathrm{~m}^{2} / \mathrm{mg} ; f=0.03 \mathrm{~Hz}$ (or $P=33.6 \mathrm{~s}$ ); $A_{0}=0.3226 \mathrm{~cm}^{2} ; A_{1}=0.0818 \mathrm{~cm}^{2}$ and $\phi=0.2276$ radian.

Figure A3.1.1 The concentration dependence of the fraction of the free interfacial area as a function of time predicted by this model.

Figure A3.1.2 The bulk concentration dependence of $\Gamma(t)$. 
Figure A3.1.3 An example of the dynamic surface tension predicted by this model.

Figure A3.1.4 An example of the fraction of the free interfacial area as function of time predicted by this model.

152

Figure A3.1.5 An example of the interfacial concentrations as a function of time.

Figure A3.1.6 An example of the model prediction of the dynamic surface tension of time.

Figure 4. 1 The cloud point temperature as functions of the mole percent of NIPAM in the copolymers. The published data are shown for comparison.

Figure 4.2 The heat of phase separation as a function of copolymer NIPAM fraction $\left(f_{\text {NIPAM }}\right.$ mol\%).

Figure 4.3 The dynamic surface tension of $1 \mathrm{wt} \%$ copolymer solutions at 25 ${ }^{\circ} \mathrm{C}$.

Figure 4. 4 The dynamic surface tension of $100 \mathrm{ppm}$ copolymer solutions at 25,40 and $45^{\circ} \mathrm{C}$.

Figure 4.5 The experimental meso-equilibrium surface tension $(\square)$ of the $1 w t \%$ aqueous copolymer solutions at $25^{\circ} \mathrm{C}$ as a function of copolymer NIPAM fraction (mol\%), the equilibrium surface tension (solid line) from Margules model (Equation 4.4) and the 
ideal equilibrium surface tension (dotted line) as defined by Equation 4.1.

Figure 4.6 Excess surface tension as a function of the mole fraction of NIPAM in the copolymer

Figure 5. 1 TEM images of the microgels. The bar in each micrograph indicate 1 micrometer in length.

210-211

Figure 5. 2 The image of M-3 microgels adsorbed at the air/water interface probed in situ by environmental scanning electron microscopy (ESEM). The temperature was controlled at $1{ }^{\circ} \mathrm{C}$ and the pressure at 5.9 Torr, which gave $100 \%$ relative humidity in the chamber.

Figure 5.3 The experimental dynamic surface tensions of the four microgel samples at bulk concentration level of $0.05 w t \%$.

Figure 5.4 The experimental dynamic surface tensions of the four microgel samples at $25{ }^{\circ} \mathrm{C}$ at bulk concentration level of $1 \mathrm{wt} \%$ and corresponding model prediction (solid lines) with $k_{l}=1.63 \times 10^{-4} \mathrm{~s}^{-1}$.

Figure 5.5 The experimental dynamic surface tensions of the four microgel samples at $40^{\circ} \mathrm{C}$ at bulk concentration level of $1 \mathrm{wt} \%$.

Figure 5.6 $t^{*}$ as a function of crosslinker (BA) concentration (wt\% of NIPAM). 
Figure 5.7 A proposed picture showing how NIPAM polymers lower the surface tension of water before the phase separation for linear polymers or volume phase transition of the microgels.

Figure 6.1 The advancing contact angle of water on cross-linked polyNIPAM gel swollen with water. The initial measurement was made at 25 ${ }^{\circ} \mathrm{C}$ and the arrows indicate the direction of temperature change.

Figure 7.1 A proposed picture showing how NIPAM polymers lower the surface tension of water before the phase separation of linear polymers or volume phase transition of the microgels.

Figure 7.2 A proposed picture showing how NIPAM polymers lower the surface tension of water after the phase separation of linear polymers or volume phase transition of the microgels.

Figure 7.3 Hydrophobic $N$-isopropyl groups in an aqueous phase have associated water molecules whereas it is proposed that surface $N$ isopropyl groups do not.

Figure 7.4 A symbolic picture describing the proposed re-arranging of an adsorbed linear NIPAM polymer chain at the oscillating air/water interface in an oscillating pendent drop experiment.

Figure 7.5 An illustration of the preferential partition of the polymerized NIPAM and AM units.

Figure 7.6 An illustration of the decrease of polymer chain mobility with the xxiii 
increase of crosslinking degree.

281

Figure 7.7 An illustration of the contact angle behavior before and after the volume phase transition of crosslinked NIPAM macrogels.

284

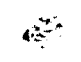

xxiv 


\section{LIST OF TABLES}

Table 1.1 Characterization methods of surface tension of liquids

14

Table 2.1 Density of PNIPAM (L-6B) aqueoussolutions as a function of temperature.

Table 2.2 Concentration dependence of the parameters describing the surface tension behavior at $25^{\circ} \mathrm{C}$ and $40^{\circ} \mathrm{C}$.

40

Table 2.3 Temperature dependence of the parameters describing the surface tension behavior of $10 \mathrm{ppm}$ aqueous solution.

Table 2.4 Parameters of the dynamic surface tension of L-6B aqueous solutions in the Oscillation Drop Volume experiment.

Table 3. 1 Solutions to $\beta(t)$ (see Equation 11 by van Eijk and Cohen [25]).

Table 4. 1 The feed ratios, copolymer NIPAM fractions (mol\%), molecular weights and distributions of the copolymers prepared in this work.

Table 4. 2 The mean sequence lengths for the copolymers.

Table 5. 1 The crosslinker $N, N^{\prime}$-methylenebisacrylamide (BA) concentration, the methods used to add the crosslinker in the microgel synthesis and the stock suspension concentration. The feed NIPAM concentration was $0.07 \mathrm{M}$ and the ammonium persulfate $1.73 \times 10^{3}$ M. The polymerization temperature was $70^{\circ} \mathrm{C}$. 
Table 5.2 Density of the aqueous microgel suspensions used in the surface tension measurement.

208

Table 5.3 The values of the theoretical hydrodynamic radius $\left(R_{h}\right)$, the radius of gyration $\left(R_{g}\right)$ and $R_{g} / R_{h}$ for the microgels in an aqueous medium at $25^{\circ} \mathrm{C}$, the intensity weighted hydrodynamic radius at $90^{\circ}$ for

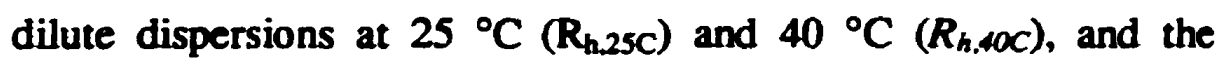
swelling ratio $\phi_{\text {swelling }}$.

214

Table 5.4 Hua-Rosen parameters $[62,63]$ describing the dynamic surface tensions of the four microgel samples and the meso-equilibrium IP group concentration at the air/water interface. 


\section{LIST OF SYMBOLS}

$\bar{M}_{w}$ the weight average molecular weight of polymers

$\bar{M}_{n} \quad$ the number average molecular weight of polymers

$\frac{\bar{M}_{w}}{\bar{M}_{n}}$ the molecular weight distribution of polymers

$R_{g} \quad$ the radius of gyration of polymers

Co the overlapping concentration of macromolecules in solution

$d$ the hydrodynamic diameters of phase-separated poly $(N$ isopropylacrylamide) (PNIPAM) particles

$R_{h} \quad$ the theoretical hydrodynamic radius of a microgel

$D_{E} \quad$ the Eff. Diam. of a microgel reported in the cumulant analysis

$R_{h, 40 C}$ the intensity-weighted hydrodynamic radius of a microgel for the incident direction of $90^{\circ}$ at $40^{\circ} \mathrm{C}$

$R_{h .2 s C}$ the intensity-weighted hydrodynamic radius of a microgel for the incident direction of $90^{\circ}$ at $25^{\circ} \mathrm{C}$ 
$\gamma_{0}$ the surface tension of pure water

$\gamma_{m} \quad$ the steady-state surface tension or the meso-equilibrium surface tension

$t^{*} \quad$ the time at which the surface tension is half way between $\gamma_{0}$ and $\gamma_{m}$

$n$ the empirical constant in Hua-Rosen Equation or the refractive index of solvent

$R^{2} \quad$ the coefficient of determination

C bulk concentration

$A(t)$ surface area of pendent drop

$A_{0} \quad$ the mean area for a sinusoidally oscillating pendent drop

$A_{1} \quad$ the amplitude for a sinusoidally oscillating drop

$a_{0}$ the initial occupied area of an adsorbed polymer chain at an interface

$a(t, u)$ the occupied area per $\mathrm{mg}$ of adsorbed polymer chains at time $t$, which were adsorbed at time $u$

$C_{b} \quad$ the bulk concentration of a polymer solution

$f$ the oscillation frequency of the drop area in the OPD experiments, $\mathrm{Hz}$;

$J$ the flux of polymers to an interface

$k \quad$ the adsorption rate constant for the EC model

$k_{1} \quad$ the adsorption rate constant in the Langmuir kinetics model 
$k_{2} \quad$ the desorption rate constant in the Langmuir kinetics model

$n_{0} \quad$ the mass of polymer per unit volume divided by the maximum mass of adsorbed polymer per unit volume, dimensionless;

$P \quad$ the period of a sinusoidal oscillation, $P=1 / f$

$t$ time

$u \quad$ any time between 0 to $t \mathrm{~s}$, i.e., the elapsed time, which is the period of time an adsorbed polymer chain has been at the interface;

$w$ the total amount of polymer chains adsorbed at the air/water interface at time $t$

$\alpha \quad$ the maximum area adsorbed polymer can expand in the EC model

$\beta \quad$ the fraction of the free interfacial area available to an incoming polymer chain, dimensionless;

$\theta \quad$ the fraction of covered interface in Langmuir kinetics model;

$x(t)$ the dynamic surface tension of a solution

$\Gamma \quad$ the interfacial concentration of adsorbed polymer chains at an interface at time $t$

$\Gamma_{\max }$ the maximum interfacial concentration of polymer

$\tau_{s} \quad$ the characteristic reconfiguration time for an adsorbed polymer chain in EC model

$r_{1}$ the monomer reactivity ratio for $N$-isopropylacrylamide (NIPAM) 
$r_{2}$ the monomer reactivity ratio for acrylamide (AM)

$\Delta H \quad$ the heat of phase separation

$\gamma_{A M}$ the meso-equilibrium surface tension of $1 \mathrm{wt} \%$ polyacrylamide aqueous solution

$\gamma_{N I P A M}$ the meso-equilibrium surface tension of 1 wt\% poly $(N-$ isopropylacrylamide aqueous solution

$f_{\text {NIPAM }}$ is the mole fraction of NIPAM moieties in a poly(NIPAM-co-AM) copolymer

$\gamma_{E X}$ the excess surface tension

$A$ the constant in the one-constant Margules model

MSL the mean sequence lengths of NIPAM sequences or AM sequences

$Z \quad$ the dissymmetry ratio

$\lambda_{0}$ the wavelength in vacuum

$I_{\theta}$ the total intensity of the light scattered by particles or microgels

D the core diameter of a microgel

$\phi_{\text {swelling }}$ the swelling ratio of a microgel 
$m_{\mathrm{gel}}$ the mass of a microgel

$C_{P} \quad$ the polymer concentration in a microgel

$N_{c}$ the average number of NIPAM repeating units between chemically bonded cross-links

$M_{\text {NIPAM }}$ the molecular weight of NIPAM repeating unit

$C_{B A}$ the concentration of the cross-linker

$M_{B A}$ the molecular weight of the cross-linker

$C_{0}$ the initial microgel mass concentration in a suspension

$V$ the volume of the pendent drop

$A$ the drop surface area

$\varepsilon \quad$ the V/A ratio of a pendent drop

$\Gamma_{\text {IP }}$ the equivalent surface concentration of $\boldsymbol{N}$-isopropyl groups 


\section{LIST OF PUBLICATIONS}

1. Zhang, Ju and Pelton, R., "The dynamic behavior of poly $(N$ isopropylacrylamide) at the air/water Interface", accepted by Colloids and Surfaces.

2. Zhang, Ju and Pelton, R., "The application of polymer adsorption models to dynamic surface tension", submitted to Lngmuir.

3. Zhang, Ju and Pelton, R., "The surface tension of aqueous poly(Nisopropylacrylamide-co-acrylamide)", accepted by J. Polym. Sci. Part A: Polymer Chemistry.

4. Chan, K.; Pelton, R. and Zhang, Ju, "On the formation of colloidally dispersed Phase-separated Poly( $N$-isopropylacrylamide)", submitted to Langmuir.

5. Wang, G.; Pelton, R. and Zhang, Ju, "Sodium dodecyl sulfate binding to poly ( $N$-isopropylacrylamide) microgel latex studied by isothermal titration calorimetry", accepted by Colloids and Surfaces.

6. Zhang, Ju and Pelton, R., "Poly $(N$-isopropylacrylamide $)$ at the air/water interface" Langmuir 12, 2611 (1996).

7. Zhang, Ju; Pelton, R. and Deng, Y., "Temperature dependent contact angles of water on poly( $N$-isopropylacrylamide) gels", Langmuir 11, 2301 (1995). 


\section{Chapter 1 Introduction}

\section{$1.1 \quad N$-Isopropylacrylamide Polymers}

N-Isopropylacrylamide (NIPAM) polymers (Figurel.1) are temperature sensitive polymers, which can be prepared in the form of homopolymers, copolymers and crosslinked gels. Linear NIPAM homopolymers are water soluble and their aqueous solutions have a lower critical solution temperature (LCST) between $30 \sim 35^{\circ} \mathrm{C}$ [1]. Compared with the LCST of the homopolymer, copolymers of $\mathrm{N}$-isopropylacrylamide and its comonomers have a lower LCST if the comonomer is more hydrophobic than NIPAM [2], or a greater LCST if the comonomer is more hydrophilic than NIPAM, such as acrylamide (AM) [3] and acrylic acid [4]. Crosslinked NIPAM gels display a corresponding volume phase transition temperature. For NIPAM microgels crosslinked by $N, N^{\prime}$. methylenebisacrylamide, their volume phase transition occurs between 30 and 34 ${ }^{\circ} \mathrm{C}$ when the crosslinker composition is varied from 0 to $15 w t \%$ of NIPAM [5]. The introduction sections in the subsequent thesis chapters present comprehensive reviews on the temperature sensitive properties of NIPAM homopolymer (Chapter 2), copolymers (Chapter 4) and crosslinked gels (Chapters 5 and 6). Figure 1.1 shows the chemical structures of the polymers studied by this thesis. 


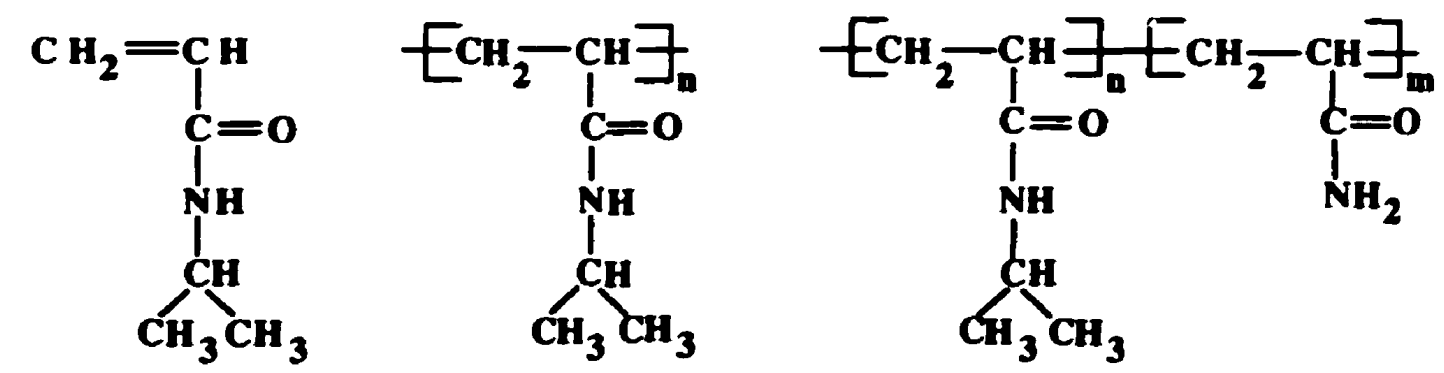

NIPAM Monomer NIPAM Homopolymer NIPAM Moiety AM Moiety

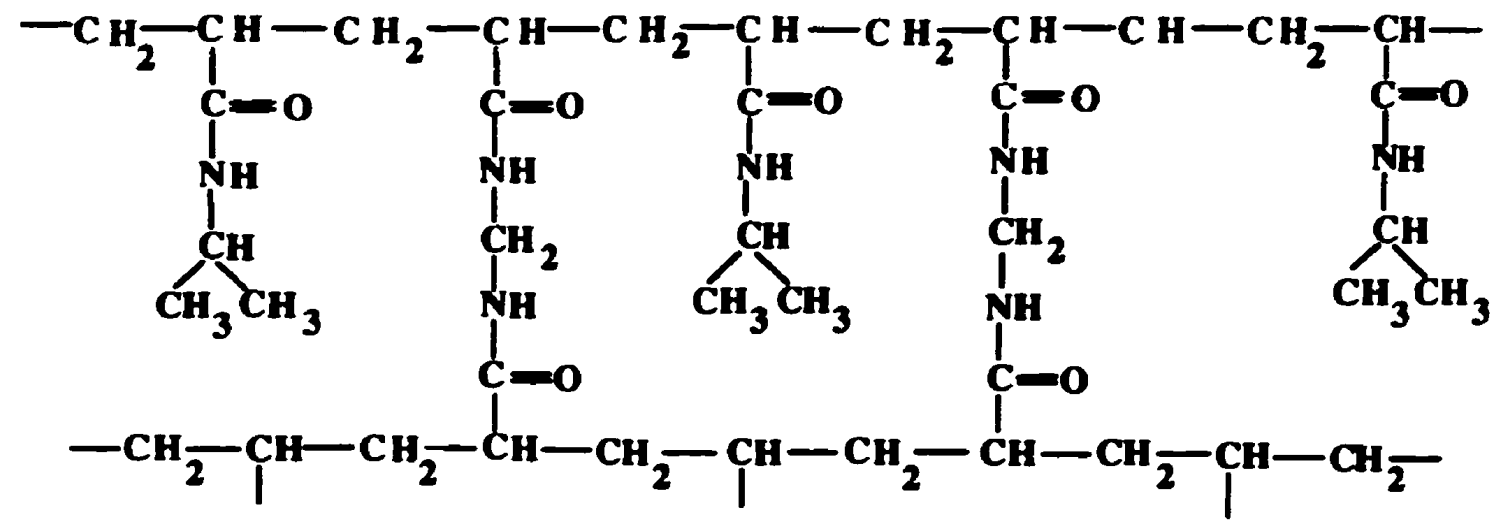

PNIPAM Crosslinked by $\mathbf{N}, N^{\prime}$-methylenebisacrylamide

Figure 1. 1 The chemical structures of NIPAM monomer, homopolymer, $\operatorname{poly}(N$ isopropylacrylamide-co-acrylamide) copolymer and NIPAM crosslinked by $N, N$ methylenebisacrylamide.

The temperature sensitivity of aqueous NIPAM polymers has evoked research into many potential applications including drug delivery systems $[6,7,8$, 9, 10, 11], protein-ligand recognition applications [12], and immunoassays [13], enhanced/tertiary oil recovery $[14,15,16,17]$, optical "switching" devices [18, 19], drag reduction [20, 21], membrane technology [22] and chromatography [23], industry separation processes [24, 25]. For many of these potential applications, the interfacial properties of NIPAM polymers are important and 
have not been studied in detail if at all. This thesis partially rectifies this situation by presenting the results of a detailed investigation of the properties of NIPAM-based materials at the air/water interface. 


\subsection{Concepts of Surface, Interface and Surface Tension}

Surface chemistry involves a broad range of disciplines having interfacial phenomena as a common thread. The terminology of the field covers a wide range of systems varying in their degree of ideality (e.g., in flatness or in uniformity). Terms such as surface, interface and surface tension are frequently used in this thesis. According to Ferguson and Whitesides [26], the term surface refers to the face of a solid (or of a liquid) directly exposed to the environment, without reference to any contacting phase; the term interface refers to the contact between a surface and a second phase (solid, liquid or gas). A relating term interphase, refers to a surface or interface and some portion of the underlying phase. For situations when the liquids adjacent to the solid can swell the surface like a polymer surface, general terms such as interfacial region are used to emphasize the complex, environmentally determined nature of the interface.

A range of experimental techniques can be used to characterize an interface, each probing a different distance scale in the interfacial region. Ferguson and Whitesides [26] define different "interphase" associated with the outer depth of the solid sensed by the various analytical tools used to probe the functionalized surface. For example, the " $\theta$ interphase" refers to the outer $5-10$ $\AA$ of a solid, the portion relevant to wetting and affecting contact angle measurement. The "XPS interphase" or "ESCA interphase" covers about the outer $50 \AA$, the portion probed by X-ray photoelectron spectroscopy. 
Surface tension is best described by Hunter [27]: "The term surface tension refers to the observation that the surface of a liquid behaves as though it were covered with a thin 'skin' which resists (to some extent) attempts to puncture it." According to Hunter, surface tension arises from the difference in energies between molecules at the surface and the molecules in the bulk phase of a material. From another point of view, surface free energy implies that work must be done to create any new surface, i.e. to bring molecules from the interior phase to the surface region. For a pure material, the specific surface free energy (energy per unit surface area) is numerically equal to surface tension (force per unit length). 


\subsection{Background Work on Water-soluble Polymers at the}

\section{Air/Water Interface}

The adsorption of water-soluble polymers at the air/water interface are involved in many natural phenomena and engineering processes, such as foaming, flocculation, emulsion and flotation. The adsorption of a water-soluble polymer at the air-water interface has been described by a number of authors $[28,29,30$, $31,32,33,34]$ and there is general agreement at the simplest level about the probable sequence of events. For dilute solutions, individual polymer chains are believed to diffuse to the interface, attach and re-configure to give an equilibrium conformation. Details of the surface configuration have been probed by modeling, neutron reflection, ellipsometry and surface tension $[35,36]$. There is a body of evidence, both theoretical and experimental, suggesting that the apparent equilibrium configuration depends upon the bulk concentration. Layers formed from relatively concentrated solutions are more extended because the available surface area is taken up before chains have a chance to spread out. Indeed, a recently published model specifically accounts for this effect $[37,38]$.

Both natural and synthetic water-soluble polymers can adsorb at the air/water interface. Proteins such as $\beta$-casein $[32,33]$ and bovine serum albumin $[39,32,33,40]$ were reported to form films at the air/water interface and lower the surface tension of water. High molecular weight hydroxypropyl cellulose, a chemically modified natural polymer which has a LCST around $40^{\circ} \mathrm{C}$, adsorbs at 
the air/water interface, giving a steady-state surface tension of about $42 \mathrm{~mJ} / \mathrm{m}^{2}$ [41]. The adsorption of poly(ethylene oxide) at the air/water interface is one of the most explored cases of water-soluble synthetic polymers. Poly(ethylene oxide) formed stable monolayer film at the air/water interface and lowered the surface tension of water $[42,43,44,45]$. Surface tensions of dilute aqueous NIPAM homopolymer solutions were measured by several groups $[46,47,48,49$, 50] and it was verified that polymerized NIPAM units were surface-active at least in the form of a homopolymer. It was also found that NIPAM homopolymers spread from its aqueous solution formed a stable layer at the air/water interface [49]. However, none of the published work from other laboratories deals with the interfacial properties of PNIPAM above the LCST, nor do they deal with the interfacial properties of copolymers or microgels. A more detailed review of the surface tension of aqueous NIPAM homopolymers is found in the introduction section of Chapter 2. 


\subsection{Background Work on Contact Angle on Water-swollen Macrogels}

The dynamic contact angle observed upon movement of the liquid front is called the advancing contact angle, $\theta_{a}$, and the angle of the receding liquid front is the receding contact angle, $\theta_{\mathrm{r}}$. Contact angle hysteresis is expressed as $\theta_{\mathrm{a}}-\theta_{\mathrm{r}}$. Both $\theta_{\mathrm{a}}$ and $\theta_{\mathrm{r}}$ are measured by direct observation of the droplet on the surface.

A water-swollen gel is called a hydrogel. In the case of a water-swollen macrogel, the surface is heterogeneous and rough, the chain segments of the crosslinked macromolecules have high mobility and have capability to rearrange their orientation at an interface according to the change of the surrounding phase, such as water or air. The high mobility and ability to re-arrange for the polymer chains between crosslinks explain the significant contact angle hysteresis existing on a hydrogel surface. This behavior of a gel surface has been verified by various reports $[51,52,53,54,55,56]$. The contact angle of a hanging air bubble beneath the hydrogel surface immersed in water is very low, indicating that the surface of the gel at the hydrogel-water interface is hydrophilic. At the same time, the contact angle of a sessile drop of water on the hydrogel-air interface is relatively high, indicating the surface of the gel at the hydrogel-air interface is relatively hydrophobic. On the basis of these observations, it is suggested that hydrophilic groups of the polymer at the hydrogel-air interface are preferentially drawn into the bulk of the hydrogel $[51,53]$. Considering free rotation around $\mathrm{C}-\mathrm{C}$ bonds 
and the long-range mobility of macromolecules, this explanation is reasonable. The main driving force for the rotation of the chain segment at the surface is the strong interaction between water and hydrophilic groups of the backbone macromolecules. Because of the driving force and the high mobility of polymer segments in a hydrogel, the rotation of the molecules or the burying of the hydrophilic groups in the hydrated bulk of the gel appears to take place faster than the time it takes to measure the contact angle [53]. When the polymer segments of a hydrogel are subjected to the asymmetric molecular force field at the geVair phase boundary, it is energetically more favorable for the segments to orient in such a way as to expose the hydrophobic side groups or the nonpolar parts of the polymer backbone toward the gaseous phase and to bury the polar sites in the aqueous phase within the gel.

There are quite a few papers on contact angle measurements on the surfaces of hydrogels other than PNIPAM $[51,52,54,55,56]$. Most of the reports are on contact angle investigations on crosslinked poly(2-hydroxyethyl methacrylate) hydrogel (PHEMA) and its derivatives, because of its significant application as hydrophilic soft contact kenses. According to the work of Hoffman et al., advancing contact angles on the HEMAVMA grafted polyethylene films in air show that as polymeric surfaces increase in hydrophilicity (with increasing fraction of HEMA), the contact angle becomes smaller for liquids such as glycerol, thiodiglycol, methylene iodide and so on. For any one surface composition, higher surface tension liquids will form larger contact angles. Their 
results of the comparison of polar/dispersion ratios of the same surfaces in air and under water supports the hypothesis that hydrophilic groups on polymeric surfaces undergo significant conformational changes to minimize surface free energies, when the environment surrounding the surface changes.

There exists contact angle measurement induced deformability of a hydrogel surface, which can lead to relatively large errors in the contact angle, particularly for low-modulus, high-water-content gels [52]. 


\subsection{The Motivation, the Theme and the Objectives of the Thesis}

Pelton has a long-term interest in the properties of NIPAM-based materials - indeed, he invented NIPAM microgels which are now the subject of intense investigation by many groups. The motivation for this work was the recognition that the interfacial properties of NIPAM-based materials were not sufficiently characterized. This is a particularly attractive system to study because NIPAM polymers range from linear polymers to macrogels and because the polymer/water interaction is so temperature sensitive.

"Poly $(\mathrm{N}$-isopropylacrylamide) at the air/water interface" is the theme of this thesis. It is polymerized NIPAM units that are the source of surface-active properties of NIPAM polymers. The objectives of the work described in this thesis are mainly to study the following ten important issues.

1. What is the quantitative relationship between surface tension and adsorption?

2. What are the factors influencing surface tension lowering and therefore the rate of adsorption?

3. How do adsorbed linear NIPAM homopolymers at air/water interface react to an interfacial area oscillation?

4. Can surface tension kinetics be modeled? 
5. How does NIPAM content in a poly(NIPAM-co-AM) copolymer affect the surface tension behavior?

6. Rigid particles (such as Talc) do not lower surface tension of water whereas soluble polymers are surface active. Do crosslinked microgels behave as particles or as polymers at the air/water interface?

7. Does the contact angle of water on a water-swollen crosslinked NIPAM macrogel equal to zero? How does the contact angle change in respond to temperature change?

8. Are the temperature sensitive solution properties of PNIPAM reflected in the properties of adsorbed polymer at the air/water interface?

9. What is the structure of the adsorbed NIPAM polymers?

10. How do the interfacial properties of NIPAM-based homopolymers, copolymers, crosslinked microgels and macrogels, compare? 


\subsection{The Surface Tension Measurement}

Surface tension measurements were chosen to probe the adsorption of NIPAM polymers at the air/water interface. Surface tension is a thermodynamic parameter requiring no models to convert raw data into surface energy. Furthermore, a big advantage of the surface tension approach is that the measurement using modern techniques can be sensitive and very rapid, and thus is suitable for kinetics study. Finally, a novel form of surface tension measurements involving oscillating pendent drops was employed to generate unique information about the dynamic behavior of the adsorption/rearrangement process. Ongoing studies using neutron reflectivity experiments have confirmed some aspects of this work. Four (Chapters 2, 3,4 and 5) out of five of the paper chapters (Chapters 2, 3, 4, 5, and 6) of this thesis employ surface tension measurement as the main experimental approach.

There are several methods that can be used to measure the surface tension of a solution [57] and are listed in Table 1. 1. Among them, the capillary rise, Wilhelmy slide and the pendent or sessile drop or bubble methods traditionally involve quiescent surfaces. Those methods are very good when used to study the slow aging effect of a liquid-air interface. The flow method and the capillary wave method are useful to study aging or relaxation effects on a very small time scale. For the maximum bubble pressure method, drop weight method and ring method, an exact edge surface is too difficult to define at the critical point, 
therefore, they are not applicable to study aging or relaxation effects on a very small time scale [57].

Toble 1. 1 Characterization methods of surface tension of liquids

Capillary rise method

Maximum bubble pressure method

Drop weight method

Ring method

Wilhelmy slide method

Methods based on the shape of static drops or bubbles

i) hanging drop method

ii) sessile drop method

iii) hanging bubble method

iv) sessile bubble method

Flow method

Capillary wave method

The technique used in this thesis is mainly the axisymmetric drop shape analysis (ADSA-P), which is the modem version of the pendent drop method. ADSA-P technique was developed in Dr. Neumann's laboratory at University of Toronto. ADSA-P is a technique that can be used to determine surface tension from the shape of the axisymmetric menisci of a pendent drop. The strategy employed is to fit the shape of a drop to the theoretical drop profile $\left(R_{1}\right.$ and $\left.R_{2}\right)$ according to the following classic Laplace equation, using surface tension as one of the adjustable parameters: 


$$
\gamma\left(\frac{1}{R_{1}}+\frac{1}{R_{2}}\right)=\Delta P \quad \text { Equation } 1.1
$$

where, $\gamma$ is the surface/interfacial tension, $R_{\mathbf{1}}$ and $\mathbf{R}_{\mathbf{2}}$ are the two principal radii of curvature and $\Delta \mathrm{P}$ is the pressure across the interface. When the orily external force is gravity, $\Delta \mathrm{P}$ is a linear function of the elevation, which is a known parameter in experiment. The best fit gives the correct or operative surface tension. The main advantages of ADSA-P over the old pendent drop method are (1) automated imaging of pendent drops and computerized analysis, and (2) surface tension measurement on both quiescent and moving pendent drops. ADSA-P allows fast and automatic surface tension measurement in a time interval less than one second. The error limits of these measurements are on the order of $0.1 \mathrm{~mJ} / \mathrm{m}^{2}$ or less. Details of the methodology can be found elsewhere [58, 59 , 60]. Figure 1. 2 shows the scheme of the surface tension measurement using ADSA-P. 


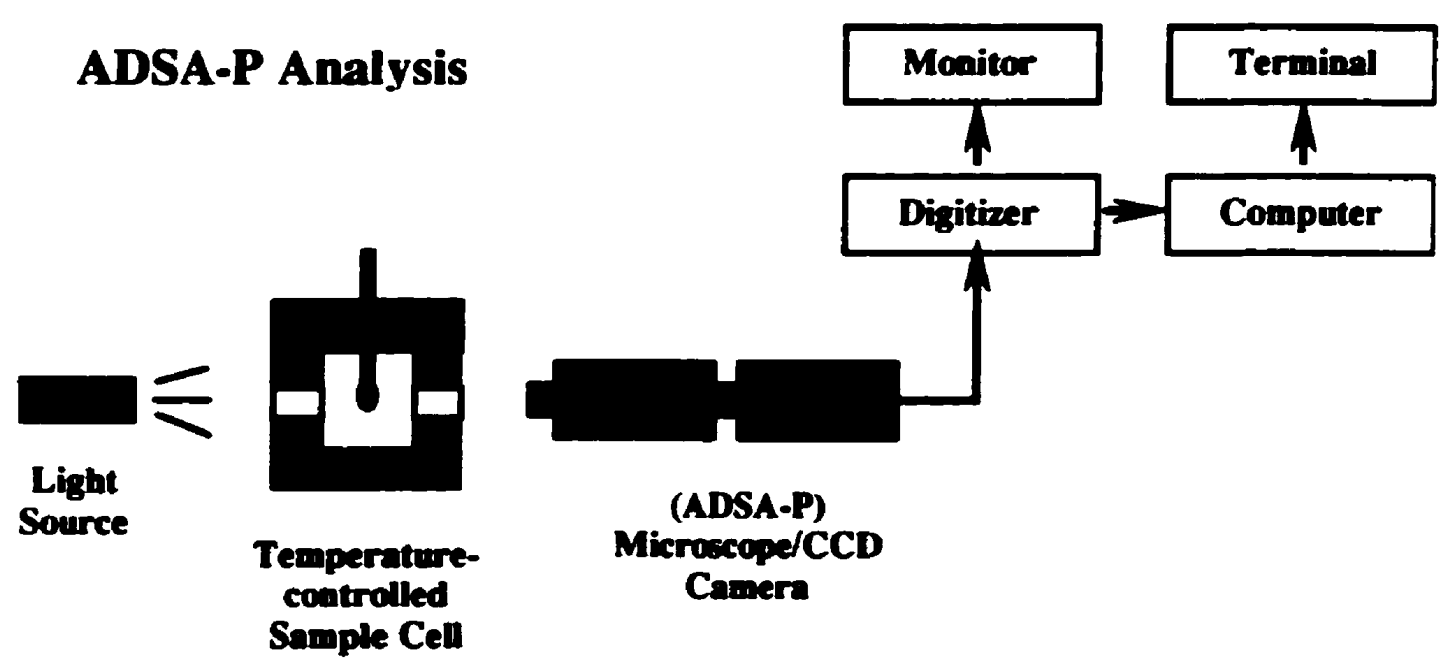

Figure 1.2 Scheme of surface tension measurement using ADSA-P.

There are other approaches that are reported to be used to study polymer adsorption at the air/water interface, which includes surface pressure measurement and optical measurements for structure properties of adsorbed polymer films.

Surface tension and surface pressure are easily convertible through the relationship: surface pressure of a solution is equal to surface tension of the solvent minus surface tension of the solution. Surface pressure measurement can be done through the Wilhelmy plate method or the Langmuir float method [61]. The Wilhelmy plate method employs a rectangular plate dipping into the air/water interface. The plate is suspended from a device measuring the downward force on the plate. Changes in the force can be directly converted to surface pressures. 
The precision of this method heavily depends on how close the contact angle of the measured liquid on the plate approaches zero, since the ideal situation is that the plate should be completely wetted by the liquid. The main problem of this method is to keep a zero contact angle. In the Langmuir float method, a horizontal rigid strip floats on the water surface at right angles to the direction of compression and is connected to the sides of a trough by flexible threads or ribbons. A torsion wire connected to the floating strip is used to detect changes in surface pressure with an increase in the coverage of water surface by a spreading polymer film. This method is flawed by the tendency of leakage of the spreading film from the floating threads or ribbons.

The optical measurement techniques include ellipsometry [34], neutron reflectivity [34] and second harmonic generation $[62,63]$. Ellipsometry measures the ellipticity of polarized light caused by reflection at an interface, which is frequently used for adsorption at liquid-solid and gas-solid interface. Ellipsometry measurement provides the refractive index and the average thickness of the adsorbed layer, and therefore, it can be used to estimate the adsorbed amount of polymer as a function of bulk phase concentration or temperature. Kawaguchi and coworkers [49] have used ellipsometry to estimate the maximum coverage of adsorbed linear NIPAM homopolymers at the air/water interface. Their data has been used to generate an empirical relationship between surface tension and surface concentration (please see Appendix 2.5 for more detail). This 
is crucial information that has been used in Chapters 2, 3 and 5 to model surface tension kinetics.

Neutron reflectivity as another important optical measurement can be used to estimate the segment density distribution of polymers at the air/water interface as a function of depth and average layer thickness. Second harmonic generation can be used to study the average molecular orientation of polymers on a substrate. 


\subsection{The Scope of the Thesis}

The work presented in this thesis focuses on investigating the surface tension behaviors of NIPAM polymers at the air/water interface. Linear NIPAM homopolymer of different molecular weights, a series of linear poly(NIPAM-coAM) copolymers of NIPAM/AM ratios between from 100:0 to $9: 91$ and crosslinked NIPAM microgels of varying crosslinking degrees were first synthesized and characterized. The dynamic surface tension behaviors of their aqueous solutions or suspensions were measured as functions of bulk concentration, temperature, molecular weight and surface area oscillation frequency, or crosslinking degree in the case of microgels. The surface tension results were analyzed at temperatures below and above the lower critical solution temperature or the volume phase transition temperature. This thesis presents the first case where the surface tension lowering abilities of both linear (homopolymer and copolymers) and crosslinked PNIPAM (microgels) were analyzed and compared. In addition, the temperature dependent contact angle of water on water-swollen crosslinked NIPAM macrogels is also the first of its kind. The temperature dependent contact angle behavior provides wetting information about the water-swollen NIPAM surface where the polymerized NIPAM backbone was fixed by chemical crosslinks. Further, attempts were made to modify and apply the generally accepted models to explain the observed surface tension behavior. 
Chapters 2, 3, describe the behavior of linear PNIPAM at the air/water interface. Chapter 4 summarizes the copolymer interfacial properties whereas Chapters 5 and 6 describe microgels and macrogels respectively. The overall conclusions are summarized in Chapter 7 together with a brief comparison of the behaviors of the different forms of PNIPAM at the air/water interface. 


\subsection{Outline of the Thesis}

In Chapter 2, the dynamic surface tension of aqueous solutions of poly $(N$ isopropylacrylamide) homopolymers are reported as functions of total polymer concentration, molecular weight, temperature and interfacial area oscillation frequency, using ADSA-P technology. The kinetic curves of the dynamic surface tension were fitted to empirical equations. To irterpret these results an empirical relationship at $25^{\circ} \mathrm{C}$ between the surface concentration and the surface tension of NIPAM homopolymers was employed. This relationship was based on data in literature or the results of a self consistent field calculation.

In Chapter 3, van Eijk and Cohen Stuar's spreading disk model was modified and applied to predict the dynamic surface tension of aqueous NIPAM homopolymer solutions, in comparison with the application of the Langmuir kinetics model. Oscillating drop experiments in which the surface area of pendent drops was varied sinusoidally were modeled. The predictions of the models on the surface tension of NIPAM homopolymer solutions were compared with the experimental data.

In Chapter 4, a series of poly( $\mathrm{N}$-isopropylacrylamide-co-acrylamide) copolymers with NIPAM to acrylamide (AM) ratios varying from $100: 0$ to $9: 91$ were synthesized through free radical polymerization and their properties were characterized by NMR, cloud points and calorimetry. Dynamic surface tensions of aqueous copolymer solutions were measured using ADSA-P technology. The 
surface tension values of copolymer solutions were predicted from the surface tensions of the homopolymers applied to a one-parameter model analogous to the Margules model for the excess free energy of mixing.

In Chapter 5, NIPAM microgels of different cross-linking degrees were synthesized. TEM and Environmental scanning electron microscopy were employed to see the shapes of the microgels and the microgels actually adsorbed at the air/water interface. The surface tension lowering abilities of the NIPAM microgels before and after the volume phase transition was verified by ADSA-P technology. For the first time, Environmental Scanning Electron Microscopy provided in situ images showing an ordered array of $500 \mathrm{~nm}$ diameter microgels adsorbed at the air/water interface.

In Chapter 6, contact angle of water on cross-linked NIPAM macrogels as a function of temperature were measured. At room temperature, the surface of water-swollen NIPAM macrogels was hydrophobic. Sessile drops of water did not spread, giving the contact angles close to $40^{\circ}$ from 25 to $35^{\circ} \mathrm{C}$ and about $90^{\circ}$ from 35 to $50^{\circ} \mathrm{C}$.

Chapter 7 concludes the findings in this thesis and presents a grand view of how NIPAM polymers adsorbed at the air/water interface and lower the surface tension of water. 


\subsection{References}

1 Schild, H. G., Prog. Polym. Sci., 17, 163 (1992).

2 Yoshida, R.; Sakai, K.; Okano, T; and Sakurai, Y., J. Biomater. Sci. Polymer Edn., 6, 585 (1994).

3 Chiklis, C. K. and Grasshoff, J. M., J. Polym. Sci.: Part A-2, 8, 1617 (1970).

4 Chen, G. and Hoffman, A. S., Macromol. Rapid Commun. 16, 175 (1995).

5 McPhee, W.; Tam, K. C. and Pelton, R. H., J. Colloid Interface Sci., 156, 24 (1993).

6 Yoshida, R.; Uchida, K.; Kaneko, Y.; Sakai, K.; Kikuchi A.; Sakurai Y. and Okano, T., Nature, 374, 240 (1995).

7 Yoshida, R.; Sakai, K; Okano T. and Sakurai, Y., J. Biomater. Sci. Polymer Edn., 6585 (1994).

8 Bae, Y. H.; Okano T. and Kim, S. W., Pharmaceutical Research, 8, 531 (1991).

9 Okano, T.; Bae, Y. H.; Jacobs, H. and Kim, S. W., J. of Controlled Release, 11, 255 (1990).

10 Dong, L. -C. and Hoffman, A. S., J. of Controlled Release, 15, 141 (1991).

11 Dong, L. -C. and Hoffman, A. S., J. of Controlled Release, 14, 21 (1990).

12 Stayton, P. S.; Shimoboji, T.; Long, C.; Chilkoti, A.; Chen, G.; Harris, J. M. and. Hoffman, A. S., Nature, 378, 472 (1995).

13 Monji, N. and Hoffman, A. S., Applied Biochemistry and Biotechnology, 13, 107 (1987).

14 Siano, D. B.; Bock. J.; Myer, P. and Valint, P. L., ACS. PMSE Prepr., 57, 609 (1987).

15 Bock, J.; Siano,D. B.; Valint, P. L. and Pace, S. J., ACS. PMSE Prepr. 57, 487 (1987). 
16 Nagarajan, R., Polym. Prepr. (Am. Chem. Soc., Div. Polym. Chem.), 22 (2), 33 (1987).

17 Khune,G. D.; Donaruma, L. G.; Hatch, M. J.; Kilmer, N. H.; Shepitka, J. S. and Martin, F. D., Polym. Prepr. (Am. Chem. Soc., Div. Polym. Chem.), 22 (2), 76 (1981).

18 Kungwatchakun, D. and Irie, M.; Makromolek. Chem., Rapid Commun., 9, 243 (1988).

19 Weissman, J. M.; Sunkara, H. B.; Tse, A. S. and Asher, S. A., Science, 274, 959 (1996).

20 Mumick, P. S. and McCormick, C. L., Polymer Engineering and Science, 34, 1419 (1994).

21 Mumick, P. S.; Hester, R. D. and McCormick, C. L., Polymer Engineering and Science, 34, 1429 (1994).

22 Nonaka, T.; Hashimoto, K. and Kurihara, S., J. Applied Polym. Sci., 66, 209 (1997).

23 Ivanov, A. E.; Zhigis, L. S.; Kurganova, E. V.; and Zubov, V. P., J. Chromatography A, 776, 75 (1997).

24 Schild, H. G., Prog. Polym. Sci., 17, 163 (1992).

25 Wang, K. L.; Burban, J. H.; Cussler, E. L.; in Responsive Gels: Volume Transitions II (Ed. K. Dusek), Berlin: Springer, 1993, p.68.

26 Ferguson, G. S. and Whitesides, G. M., Modern Approaches to Wettability,Theory and Applications (edited by Malcolm E.Schrader and Georg I.Loeb), Plenum Press, New York and London ,1994, p.144.

27 Hunter, R., Introduction to Modern Colloid Science, Oxford Science Publications, New York, 1994, pp.132-133.

28 Milner, S. R., Philos. Mag., 13, 96 (1907).

29 Langmuir, I. and Schaefer, V. J., J. Am. Chem. Soc., 59, 2400 (1937).

30 Cumper, C. W. N. and Alexander, A. E., Rev. Pure Appl. Chem., 1, 121 (1951). 
31 MacRitchie, F. and Alexander, A. E., J. Colloid Sci., 18, 453 (1963).

32 Tripp, B. C.; Magda, J. J. and Andrade, J. D., J. Colloid Interface Sci. 173, 16 (1995).

33 Graham, A. D. E. and Phillips, M. C. J. Colloid Interface Sci., 70, 403 (1979).

34 Kawaguchi, M., Prog. Polym. Sci., 28, 341 (1993).

35 Fleer, G. J.; Cohen Stuart, M. A.; Scheutjens, J. M. H. M.; Cosgrove, T. and Vincent, B., Polymers at the Interfaces, Chapman \& Hall, first edition, 1993.

36 Cohen Stuart, M. A. and Fleer, G. J., Annu. Rev. Mater. Sci., 26, 463 (1996).

37 Cosgrove, T.; Prestidge, C. A.; King, S. M. and Vincent, B., Langmuir, 8, 2206 (1992).

38 Cosgrove, T.; Prestidge, C. A. and Vincent, B., J. Chem. Soc. Faraday Trans., 86, 1377 (1990).

39 Miller, R.; Policova, Z.; Sedev, R. and Neumann, A. W., Colloids Surfaces A: Physicochem. Eng. Aspects, 76, 179 (1993).

40 Paulsson, M., and Dejmek, P., J. Colloid Interface Sci. 150, 394 (1992).

41 Chang, S. A. and Gray D. G., J. Colloid Interface Sci. 67, 255 (1978).

42 Shuler, R. L. and Zisman, W. A., J. Phys. Chem., 74, 1523 (1970).

43 Kawaguchi, M.; Komatsu, S.; Matsuzumi, M. and Takahashi, A., J. Colloid Interface Sci. 102, 356 (1984).

44 Kuzmenka, D. J. and Granick, S., Polym. Commun., 29, 64 (1988).

45 Kuzmenka, D. J. and Granick, S., Macromolecules, 21, 779 (1988).

46 Schild, H. G. and Tirrell, D. A., Langmuir, 7665 (1991).

47 Fujishige, S.; Koiwai, K.; Kubota, K. and Ando, I., Kenkyu Hokoku-seni Kobunshi Zairyo Konkyusho, 167, 47 (1991).

48 Zhang, Ju and Pelton, R., Langmuir, 12, 2611 (1996). 
49 Kawaguchi, M.; Saito, W. and Kato, T., Macromolecules, 27, 5882 (1994).

50 Kawaguchi, M.; Hirose, $Y$ and Kato, T., Langmuir, 12, 3523 (1996).

51 Holly, F. J. and Refojo, M. F., J. Biomed. Mater. Res. 9, 315(1975).

52 Andrade, J. D.; King, R. N.; Gregonis, D. E. and Coleman, D. L., J. Polym. Sci.: Polym. Symp. 66, 313(1979).

53 Yasuda, H. and Sharma, Ashok K., J. Polym Sci.: Polym. Phys. Ed. 19, 1285 (1981).

54 Lee, S. H. and Ruckenstein, E., J. Colloid Interface Sci. 120, 529 (1987).

55 Ko, Y. C.; Ratner, B. D. and Hoffman, A. S., J.Colloid Interface Sci. 82(1), 25 (1981).

56 King, R. N.; Andrade, J. D.; Ma, S. M.; Gregonis, D. E. and Brostrom, L. R., J. Colloid Interface Sci. 103(1), 62 (1985).

57 Adamson, A. W., Physical Chemistry of Surfaces, third edition, John Wiley \& Sons, Inc., 1976.

58 Rotenberg, L.; Boruvka, Y.; Neumann, A. W., J. Colloid Interface Sci., 93, 169 (1983).

59 Kwok, D. Y.; Vollhardt, D.; Miller, R.; Li., D. and Neumann, A. W., Colloids and Surfaces A: Physicochemical and Engineering Aspects, 88, 51 (1994).

60 Kwok, D., Masters Thesis, University of Toronto, 1994.

61 MacRitchie, F., Chemistry at Interfaces, Academic Press, San Diego (1990).

62 Shen, Y. R., Nature, 37, 519 (1989).

63 Corn, R. M., Anal. Chem., 63, 285A (1991). 


\title{
Chapter 2
}

\section{The Dynamic Behavior of Poly(N-isopropylacrylamide) at the Air/Water Interface}

\begin{abstract}
The dynamic surface tension of poly( $N$-isopropylacrylamide) (PNIPAM) homopolymers has been studied as functions of total polymer concentration, molecular weight, temperature and interfacial area oscillation frequency. PNIPAM lowers the surface tension of water both below and above the LCST. In the latter case, colloidal polymer particles in the bulk phase diffuse to the interface to unwrap and spread to lower surface tension. The time scale for surface tension lowering decreases with increasing polymer concentration and decreasing molecular weight in the range from 13,100 to 547,000 . The kinetics of surface tension lowering were not very sensitive to temperature for the lower molecular weight $\left(1.31 \times 10^{4}\right)$ PNIPAM in contrast to the behavior of the higher $\left(5.47 \times 10^{5}\right)$ MW polymer. The kinetic curves were fitted to the Hua-Rosen equation yielding a minimum meso-equilibrium (steady-state) surface tension of about $43 \mathrm{~mJ} / \mathrm{m}^{2}$ at 25 ${ }^{\circ} \mathrm{C}$. The meso-equilibrium surface tension showed a slight $\left(-0.3 \mathrm{~mJ} / \mathrm{m}^{2}{ }^{\circ} \mathrm{C}\right)$ decrease with increasing temperature with the most dramatic changes occurring
\end{abstract}


around the LCST.

Surface tension was measured as a function of time under conditions where the pendent drop surface area varied sinusoidally. At long time, oscillations of the pendent drop surface area induce small surface tension oscillations and the amplitude of a surface tension oscillation at long time is a function of the extent of surface dilation, which reflects the ability of loops to become trains when the surface expands.

Key Words: Poly( $N$-isopropylacrylamide), dymamic surface tension, surface dilation, air/water interface. 


\subsection{Introduction}

Poly(N-isopropylacrylamide) (PNIPAM) is a water soluble polymer with a lower critical solution temperature (LCST) between 30 and $35^{\circ} \mathrm{C}$, depending upon the detailed microstructure of the macromolecule [1]. Below this temperature, PNIPAM exists as soluble coils in aqueous media. Upon heating through the LCST, PNIPAM chains undergo a coil-to-globule transition and collapse to form phase-separated globules $[2,3,4]$ which aggregate to form stable colloidal suspensions or macroscopic precipitates [5]. The temperature sensitivity of aqueous PNIPAM has spurred research into many potential applications including drug delivery systems $[6,7,8,9,10,11]$, protein-ligand recognition applications [12], and immunoassays [13]. This paper addresses the properties of PNIPAM at the air/water interface, which are interesting from a scientific perspective and may influence potential applications.

Surface tensions of dilute aqueous PNIPAM solutions have been measured by several groups $[14,15,16,17]$. Fujishige et al [15] used the Whilhelmy method for $0.25-1 \mathrm{w} / \mathrm{v} \%$ PNIPAM which gave surface tension values of 47.8 $\mathrm{mJ} / \mathrm{m}^{2}$ at $31^{\circ} \mathrm{C}$ compared with $41.9 \mathrm{~mJ} / \mathrm{m}^{2}$ at an unspecified temperature above the LCST.

Kawaguchi and coworkers [18] spread PNIPAM on a Langmuir trough and measured surface concentration and adsorbed layer thickness by ellipsometry. 
In addition, they measured the surface pressure. They reported their results as functions of the "spread amount", which was the quantity of polymer per unit surface area added to the trough. The spread amount was higher than the surface concentration measured by ellipsometry presumably since some of the PNIPAM transferred from the interface to the bulk solution. Surprisingly, this group did not relate the surface tension results to the measured surface concentrations; this analysis is reported herein (See Equation 2.4 in discussion).

Kawaguchi et al.'s work indicated that the amount of polymer at the air/water interface approximately doubled when the temperature increased from 16 to $31.3^{\circ} \mathrm{C}$ [18]. As PNIPAM approached the LCST, the coil diameter decreased, which may account for the higher surface concentrations at $31.3^{\circ} \mathrm{C}$. The amount of PNIPAM at the air water interface was similar to that measured on the silica/water interface [19].

Recently, the same group [17] published a paper reporting effects of temperature and concentration on surface tension of aqueous PNIPAM. Their measurements covered the concentration range $0.0030 \mathrm{~g} /(3.0 \mathrm{ppm})$ to $0.010 \mathrm{~g} / \mathrm{L}$ (10 ppm) at $16^{\circ} \mathrm{C}, 31.3{ }^{\circ} \mathrm{C}$ and $34.8{ }^{\circ} \mathrm{C}$ respectively. They reported mesoequilibrium (i.e., steady-state) surface tension values of $45.6 \mathrm{~mJ} / \mathrm{m}^{2}$ at $16^{\circ} \mathrm{C}, 40.7$ $\mathrm{mJ} / \mathrm{m}^{2}$ at $31.3^{\circ} \mathrm{C}$ and $40.5 \mathrm{~mJ} / \mathrm{m}^{2}$ at $34.8{ }^{\circ} \mathrm{C}$. Furthermore, they concluded that the meso-equilibrium surface tension was independent of bulk concentration at a 
fixed temperature whereas the time required to reach meso-equilibrium decreased with increase of polymer concentration.

In earlier work, we showed that upon heating a pendent drop saturated with adsorbed PNIPAM through the LCST, there was no significant discontinuity of surface tension change corresponding to the bulk phase separation [16]. In this work we use the Neumann's automated pendent drop apparatus $[20,21,22]$ to record dynamic surface tensions as functions of time, temperature, PNIPAM concentration and molecular weight. Additionally, we report the effects of dilation and compression on the dynamic surface tension. 


\subsection{Experimental}

\subsection{Synthesis and Purification}

Two polymer samples were prepared. $40 \mathrm{~g} N$-isopropylacrylamide (Kodak Co.), double recrystallized from toluene/hexane, was dissolved in $400 \mathrm{ml}$ Milli $\mathrm{Q}$ water at room temperature under $\mathbf{N}_{2}$. The polymerization was initiated with ammonium persulfate ( $0.04 \mathrm{~g}$ for $\mathrm{L}-6 \mathrm{~B}$ and $4.0 \mathrm{~g}$ for $\mathrm{L}-4)$ and sodium metabisulfite ( $0.04 \mathrm{~g}$ for $\mathrm{L}-6 \mathrm{~B}$ and $4.0 \mathrm{~g}$ for $\mathrm{L}-4$ ). After 25 hours, the solution was heated to about $40{ }^{\circ} \mathrm{C}$ to precipitate the polymer. The polymer was dissolved and precipitated from water twice, then the polymer solution was dialyzed $(12,000$ $14,000 \mathrm{M} . \mathrm{W}$. cutoff) until the conductivity of the dialysate was less than $1 \mu \mathrm{s} / \mathrm{cm}$. Finally, the water was evaporated and the two polymer samples were dried to constant weight at $60^{\circ} \mathrm{C}$ under vacuum

\subsubsection{Characterization}

The weight average molecular weight $\left(\bar{M}_{w}\right)$ and the molecular weight distribution $\left(\frac{\bar{M}_{w}}{\bar{M}_{n}}\right)$ of the polymer were characterized by gel permeation chromatography (GPC) using PLgel $500 \AA$ column, DMA as solvent and a flow rate of $1 \mathrm{~cm}^{3} / \min$ at $20^{\circ} \mathrm{C}$. The weight average molecular weight $\left(\bar{M}_{w}\right)$ was $5.47 \times 10^{5}$ for L-6B and $1.31 \times 10^{4}$ for L-4, respectively and the corresponding 
polydispersity indices were 1.76 for L-6B and 3.63 for L-4.

Kubota et al. [3] reported the radius of gyration for two PNIPAM samples and their data are fitted at $20{ }^{\circ} \mathrm{C}$ to get the following expression (see Appendix 2.1):

$$
R_{g}=1.1 \times 10^{-5} \bar{M}_{w}^{0.59}
$$

\section{Equation 2.1}

where $R_{g}(\mathrm{~nm})$ is the radius of gyration. The $R_{g}$ values for L-4 and L-6B are respectively $11.5 \mathrm{~nm}$ and $26.8 \mathrm{~nm}$ The concentration at which the chains overlap (Co) was estimated by assuming that the chains occupy a spherical volume with radius $R_{g}$ [3]. The $C_{o}$ values are $34.2 \mathrm{ppm}$ for $\mathrm{L}-4$ and $11.3 \mathrm{ppm}$ for $\mathrm{L}-6 \mathrm{~B}$ (see Appendix 2.1).

\subsubsection{Dynamic Light Scattering}

The aggregate sizes of phase-separated PNIPAM were measured as a function of concentration by dynamic light scattering. A Brookhaven Model BI9000AT correlator and a Lexel $(25 \mathrm{~mW})$ argon laser operating with an incident wavelength of $514 \mathrm{~nm}$ were employed with detection at a scattering angle of $90^{\circ}$. The data were processed by the cumulant analysis.

\subsubsection{Surface Tension Measurement}

Dynamic surface tensions were measured by pendent drop experiments 
using axisymmetric drop shape analysis (ADSA-P) at the University of Toronto. ADSA-P is a technique that can be used to determine surface tensions from the shape of the axisymmetric menisci of a pendent drop. The strategy employed is to fit the shape of a drop to the theoretical drop profile according to the Laplace equation, using surface tension as one of the adjustable parameters. The best fit gives the correct or operative surface tension. The error limits of these measurements are on the order of $0.1 \mathrm{~mJ} / \mathrm{m}^{2}$ or less (see Appendix 2.2 for examples of drop profiles and corresponding surface tensions). The ADSA analysis required accurate density measurements, which were made as functions of temperature and polymer concentration with an AP PAAR DMA45 MC 1296 densitometer. Some of the density results are summarized in Table 2. 1. Pendent drops were formed on the tip of a Teflon capillary with an outside diameter of 0.1 in. and inside diameter of 0.076 in. (Chromatographic Specialties Inc.). Fluid flow to the capillary was controlled with a Hamilton Gastight syringe (Chromatographic Specialties Inc.) driven by a Model 18515 stepper motor (Oritel Corp.). For some experiments the volume of the pendent drop was altemately increased and decreased linearly with time. The pendent drop was enclosed in a sealed quartz cuvette (Hellma $330984,10 \times 10 \times 30 \mathrm{~mm}^{3}$ ), which was mounted in a Model 100-07 environmental chamber (Rame Hart Inc.). The temperature control was $\pm 0.1{ }^{\circ} \mathrm{C}$. Further details of the apparatus and methodology can be found elsewhere $[20,21$. 22]. The water used to prepare the solutions had a surface tension of $72.20 \mathrm{~mJ} / \mathrm{m}^{2}$ 
at $25^{\circ} \mathrm{C}$ in good agreement with $72.14 \mathrm{~mJ} / \mathrm{m}^{2}$ given by Jasper [23].

Table 2. 1 Density of PNIPAM (L-6B) aqueoussolutions as a function of temperature.

\begin{tabular}{|c|ccc|}
\hline $\left.\mathrm{T}^{\circ} \mathrm{C}\right)$ & $10 \mathrm{ppm}$ & $1 \%$ & $10 \%$ \\
\hline 25 & 0.997 & 0.9984 & 1.0105 \\
30 & 0.9957 & 0.9969 & 1.0084 \\
33 & 0.9949 & 0.9962 & 1.0078 \\
34 & 0.9944 & 0.9957 & 1.0072 \\
35 & 0.994 & 0.9951 & 1.0051 \\
37 & 0.9933 & 0.9942 & 1.0037 \\
40 & 0.9924 & 0.9933 & 1.0026 \\
\hline
\end{tabular}




\subsection{Results}

\subsubsection{Dynamic light scattering}

Above the LCST, PNIPAM is present in water as colloidally dispersed particles. Figure 2. 1 shows the hydrodynamic diameters of phase-separated PNIPAM as functions of the bulk concentration at $40{ }^{\circ} \mathrm{C}$. L-4 produced slightly larger particles than did L-6B. For both polymers the average particle size increased with polymer concentration.

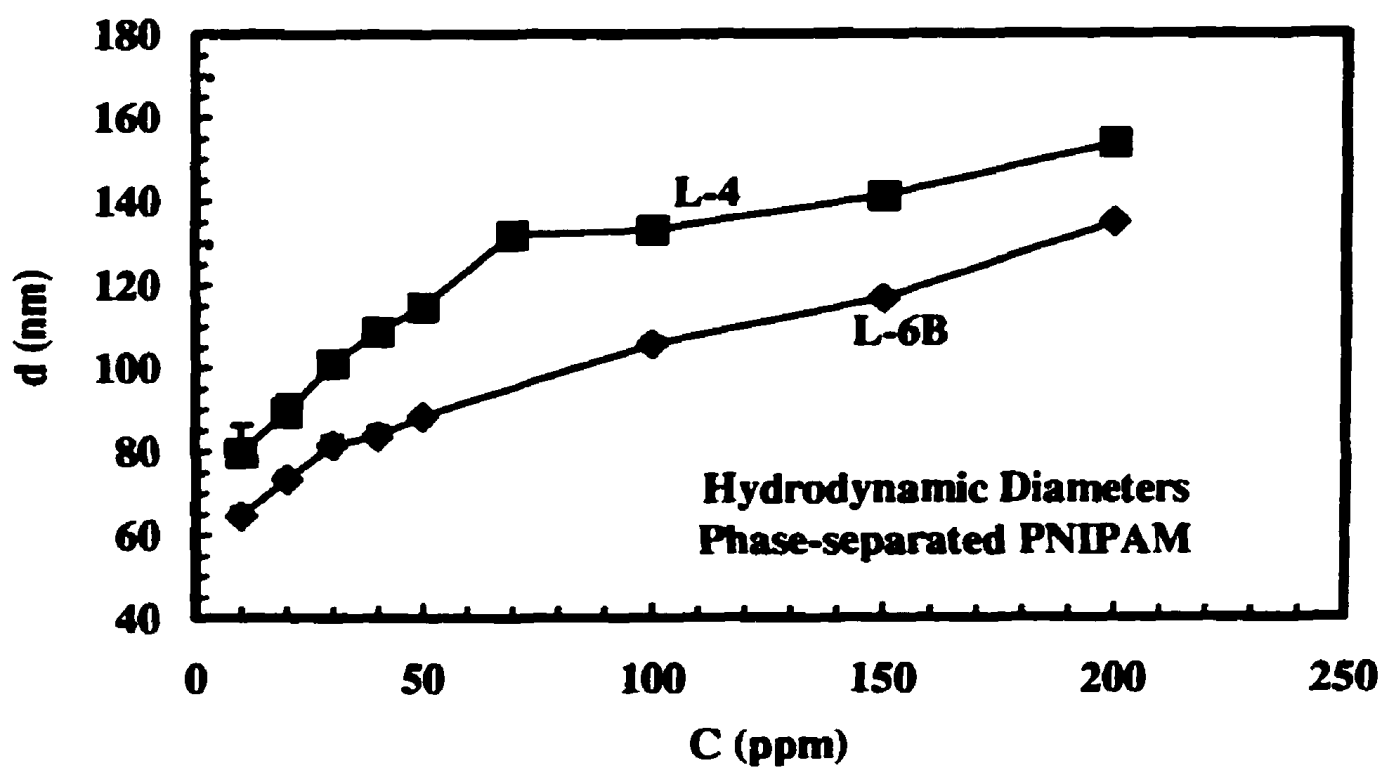

Figure 2. 1 The hydrodynamic diameters of phase-separated PNIPAM colloidal aggregates at $40^{\circ} \mathrm{C}$ as a function of the solution concentration before phase separation. (The error bars indicate the relative standard deviations (it is between 1.02 to $2.45 \%$ for $\mathrm{L}$ $6 B$ and 1.69 to $6.66 \%$ for $L-4$ ). 


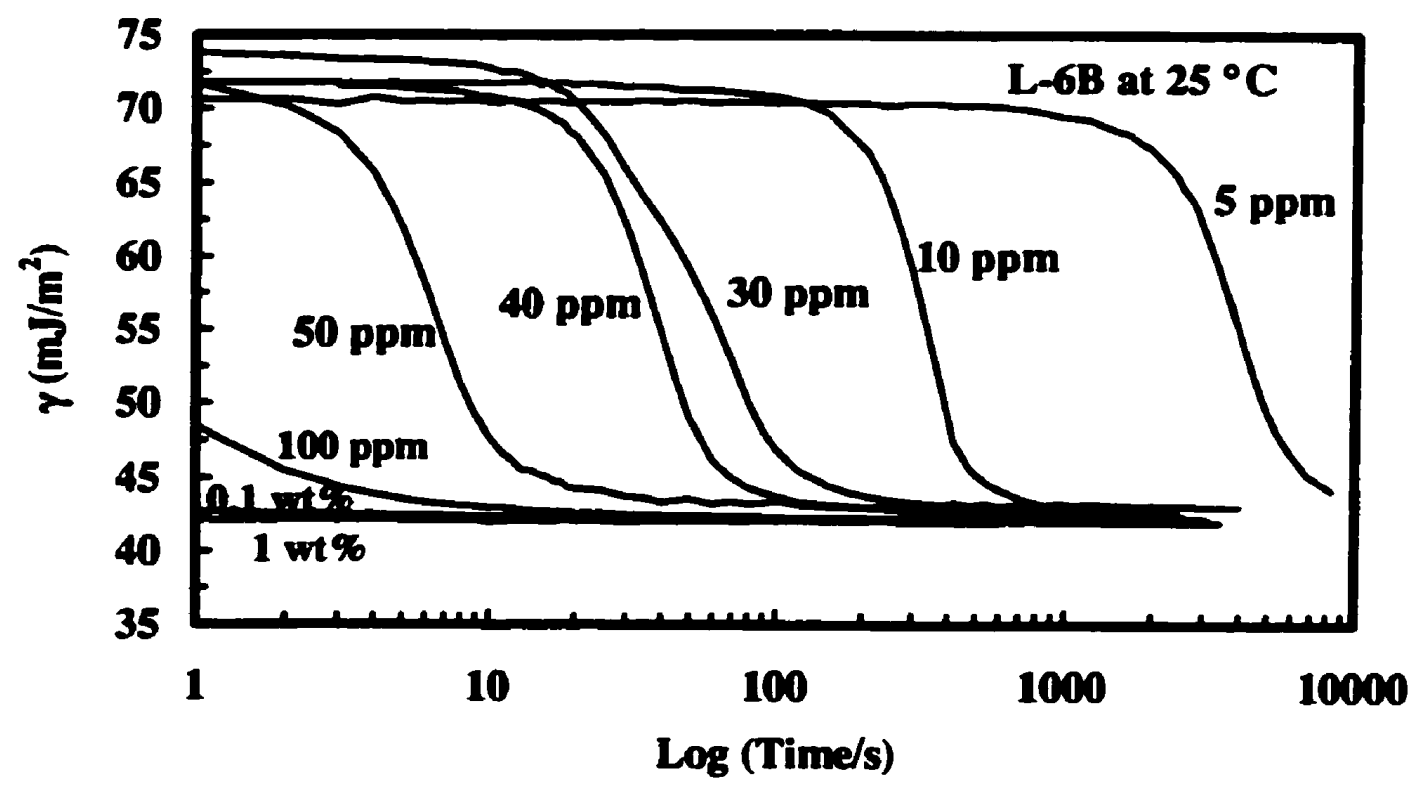

Figure 2. 2 The concentration dependence of the dynamic surface tension of PNIPAM (L-6B) aqueous solutions at $25^{\circ} \mathrm{C}$.

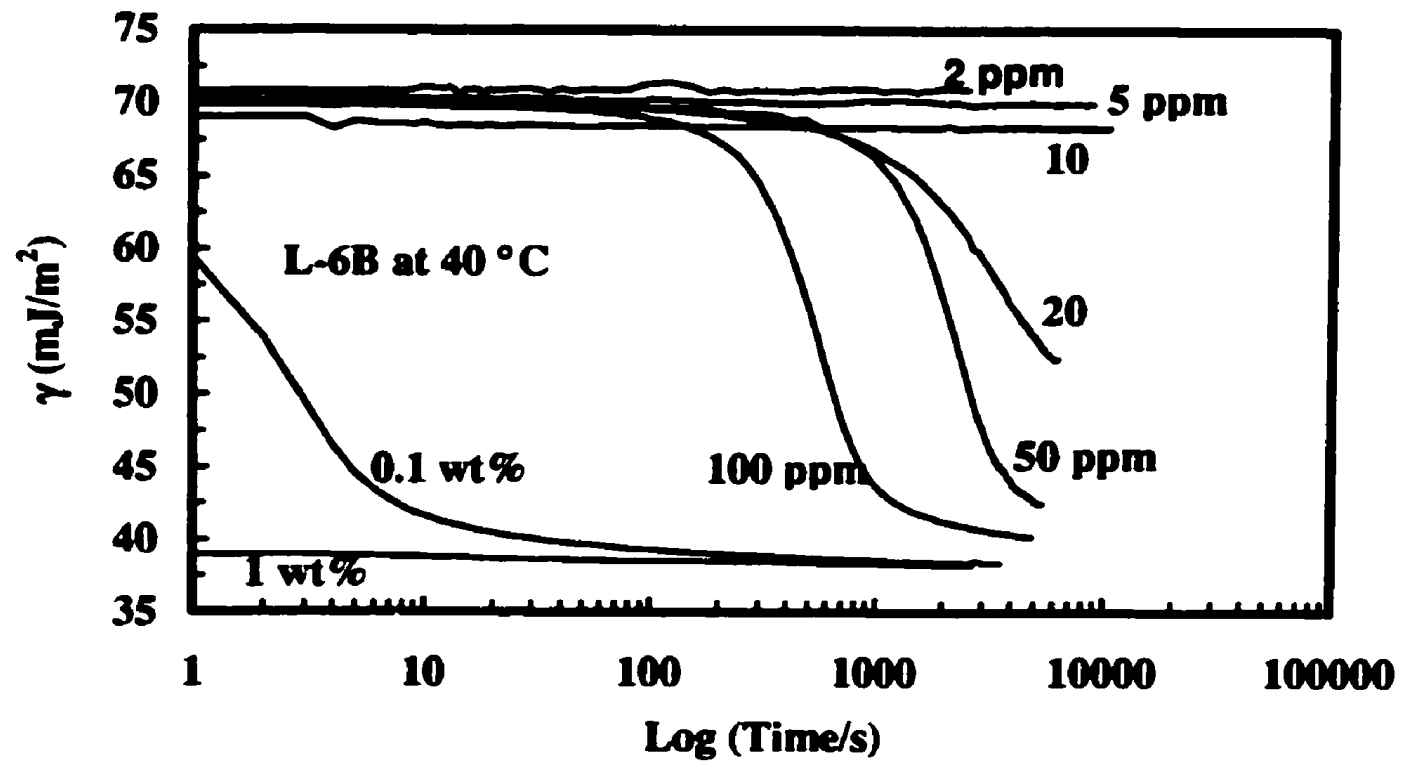

Figure 2.3 The concentration dependence of the dynamic surface tension of PNIPAM (L-6B) aqueous solutions at $40^{\circ} \mathrm{C}$. 


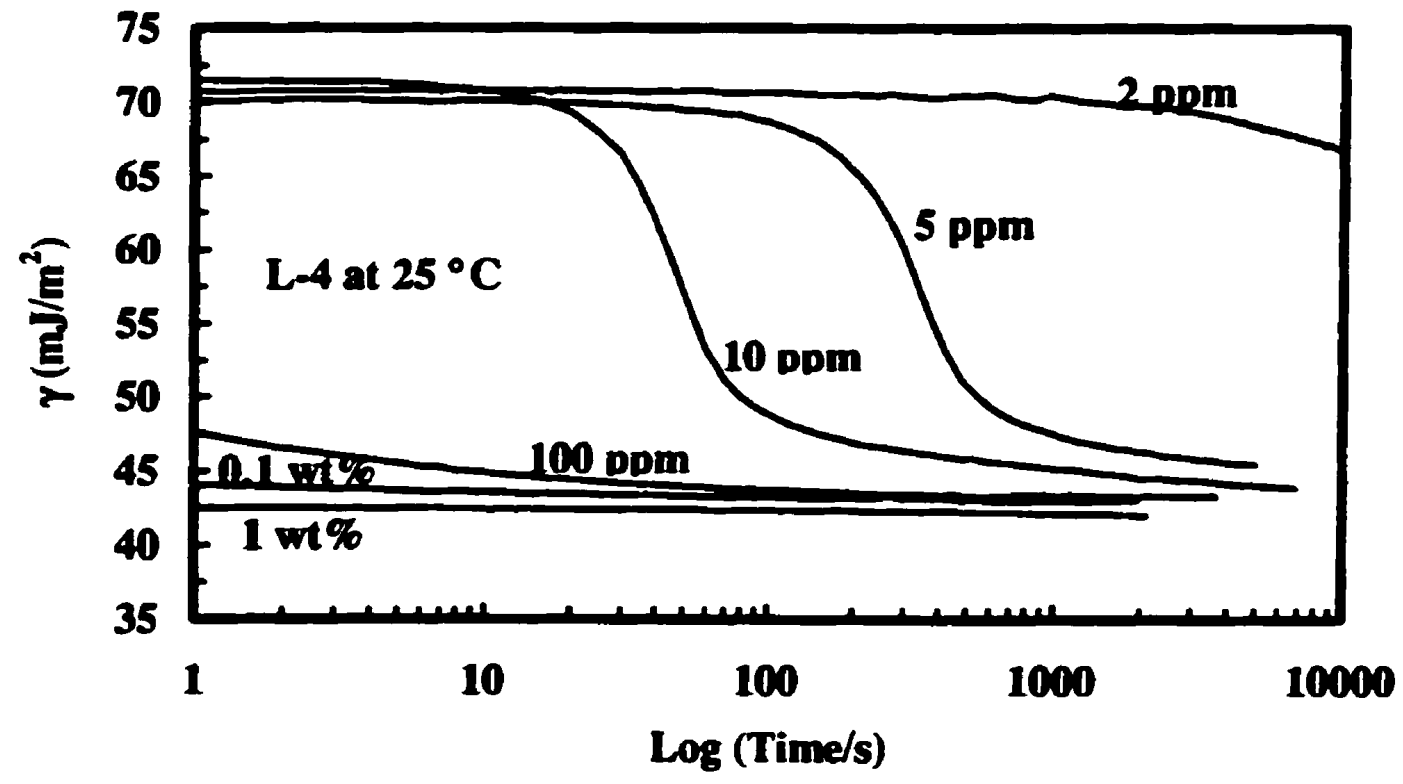

Figure 2.4 The concentration dependence of the dynamic surface tension of PNIPAM (L-4) aqueous solutions at $25^{\circ} \mathrm{C}$.

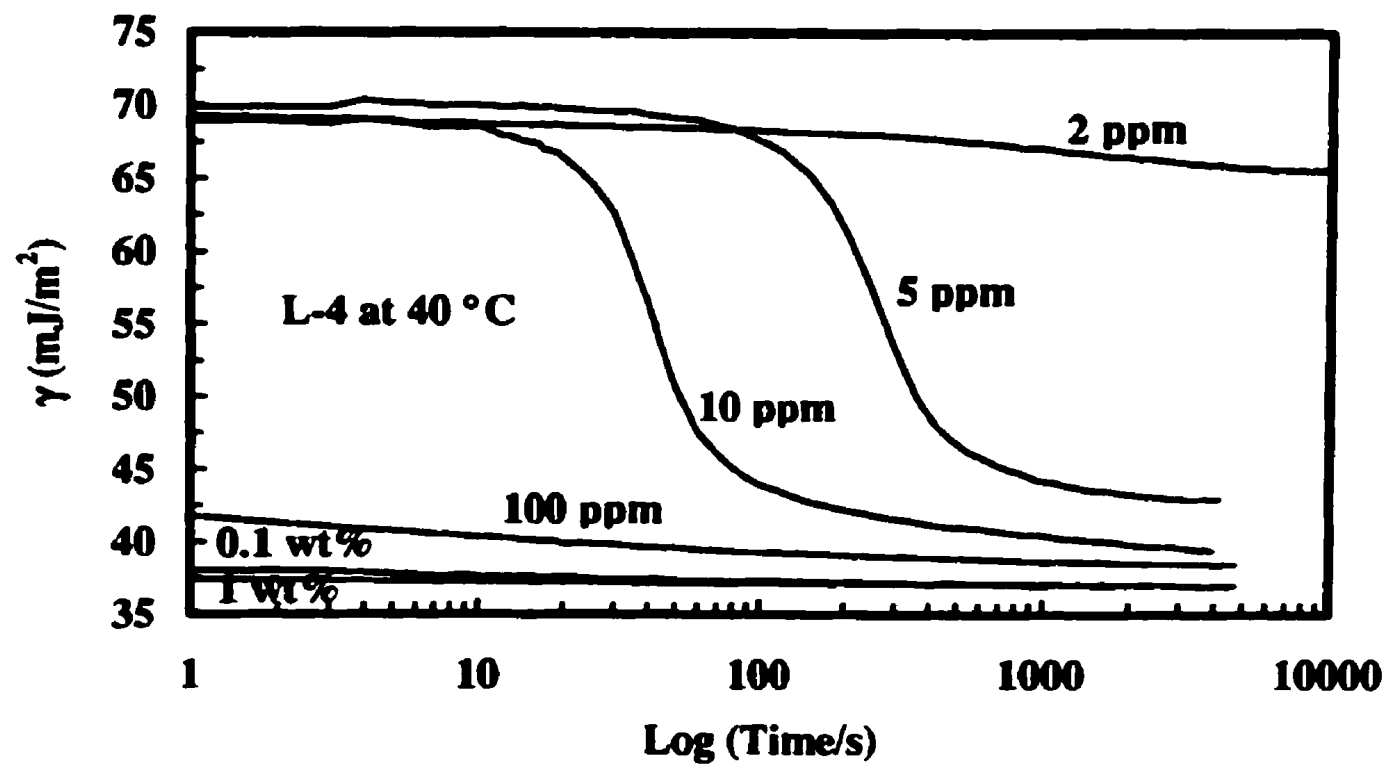

Figure 2.5 The concentration dependence of the dynamic surface tension of PNIPAM (L-4) aqueous solutions at $40^{\circ} \mathrm{C}$. 


\subsubsection{Static Drop Volume Experiment}

Surface tension values were measured as function of time for various concentrations of L-4 and L-6B at 25 and $40^{\circ} \mathrm{C}$ and the results are summarized in Figure 2. 2 to Figure 2. 5. Each curve is based on hundreds of data points and the results were fitted to the empirical Hua-Rosen equation $[24,25]$ given below:

$$
\log \frac{\gamma_{0}-\gamma(t)}{\gamma(t)-\gamma_{0}}=n \log \frac{t}{t^{*}}
$$

Equation 2.2

where, $y(t)$ is the surface tension at time $t, \gamma_{0}$ is the surface tension at the instant the drop is formed which is assumed to equal to the surface tension of pure water, $n$ is an empirical constant, $\gamma_{m}$ is the steady-state surface tension which Hua and Rosen call the "meso-equilibrium" surface tension, and $t^{*}$ is the time at which the surface tension is half way between $\gamma_{0}$ and $\gamma_{m}$. The Hua-Rosen equation was fitted to the experimental data in Figures 2 to 5 by non-linear regression (using the Solver in Microsoft ${ }^{\circ}$ Excel 97, see Appendix 2.4) and the results are summarized in Table 2. 2 and Table 2. 3. For both PNIPAM samples at both temperatures the results in Figure 2. 2 to Figure 2.5 show that the rate of surface tension lowering increases with polymer concentration. 
Table 2. 2 Concentration dependence of the parameters describing the surface tension behavior at $25^{\circ} \mathrm{C}$ and $40^{\circ} \mathrm{C}$.

\begin{tabular}{|c|c|c|c|c|c|c|}
\hline Sample & $\begin{array}{c}\mathrm{T} \\
\left({ }^{\circ} \mathrm{C}\right)\end{array}$ & $\begin{array}{c}C \\
(\mathrm{ppm})\end{array}$ & $n$ & $\begin{array}{c}\gamma_{m} \\
\left(m \mathrm{~m} / \mathrm{m}^{2}\right)\end{array}$ & (s) & $\boldsymbol{R}^{2}$ \\
\hline \multirow{4}{*}{$L-4$} & \multirow[t]{2}{*}{25} & 5 & 2.7481 & 45.72 & 330.1 & 0.9992 \\
\hline & & 10 & 2.4679 & 44.46 & 49.6 & 0.9963 \\
\hline & \multirow[t]{2}{*}{40} & 5 & 2.7259 & 43.10 & 258.7 & 0.9994 \\
\hline & & 10 & 2.4489 & 40.25 & 42.4 & 0.9961 \\
\hline \multirow{7}{*}{ L-6B } & \multirow[t]{5}{*}{25} & 5 & 2.9160 & 43.30 & 3694.4 & 0.9818 \\
\hline & & 10 & 4.2017 & 43.25 & 422.1 & 0.9985 \\
\hline & & 30 & 2.4113 & 43.10 & 56.8 & 0.9993 \\
\hline & & 40 & 3.6673 & 43.02 & 35.5 & 0.9999 \\
\hline & & 50 & 2.9947 & 43.14 & 6.0 & 0.9984 \\
\hline & \multirow[t]{2}{*}{40} & 50 & 2.8328 & 40.18 & 2092.7 & 0.9991 \\
\hline & & 100 & 2.7974 & 40.36 & 508.2 & 0.9995 \\
\hline \multicolumn{7}{|c|}{ Note: $R^{2}=1-\frac{\sum_{0}^{k}\left(\gamma_{i}-\gamma_{i, f}\right)^{2}}{\sum_{0}^{k}\left(\gamma_{i}\right)^{2}-\frac{\left(\sum_{0}^{k} \gamma_{i}\right)^{2}}{k+1}}$, where $\gamma_{i}$ is the experimentally measured } \\
\hline
\end{tabular}

surface tension and $\gamma_{i f}$ is the fitted value. 
Table 2. 3 Temperature dependence of the parameters describing the surface tension behavior of 10 ppm aqueous solution.

\begin{tabular}{|c|c|c|c|c|c|}
\hline Somple & $\mathrm{T}\left({ }^{\circ} \mathrm{C}\right)$ & $n$ & $\gamma_{m}\left(m \mathrm{~J} / \mathrm{m}^{2}\right)$ & $F(s)$ & $R^{2}$ \\
\hline \multirow{8}{*}{ L-4 } & 25 & 2.4679 & 44.46 & 49.6 & 0.9963 \\
\hline & 30 & 2.8591 & 43.95 & 52.6 & 0.9977 \\
\hline & 32 & 3.0993 & 43.79 & 50.2 & 0.9980 \\
\hline & 33 & 2.9321 & 42.74 & 46.3 & 0.9974 \\
\hline & 34 & 2.2290 & 41.97 & 25.4 & 0.9965 \\
\hline & 35 & 2.1280 & 41.65 & 23.6 & 0.9963 \\
\hline & 37 & 2.7187 & 41.18 & 40.0 & 0.9970 \\
\hline & 40 & 2.4489 & 40.25 & 42.4 & 0.9961 \\
\hline \multirow{5}{*}{$L-6 B$} & 25 & 4.2017 & 43.25 & 422.1 & 0.9885 \\
\hline & 30 & 3.8789 & 41.78 & 417.2 & 0.9988 \\
\hline & 33 & 4.0618 & 40.58 & 521.0 & 0.9943 \\
\hline & 34 & 3.9675 & 40.53 & 441.1 & 0.9962 \\
\hline & 35 & 2.4442 & 40.84 & 675.4 & 0.9988 \\
\hline
\end{tabular}

To see more clearly the effect of bulk polymer concentration on surface tension kinetics, $t^{*}$ was plotted as a function of concentration in Figure 2. 6. The $t^{*}$ values for PNIPAM L-6B decreased over more than two order's magnitude when the polymer concentration was increased from 5 to $50 \mathrm{ppm}$. Molecular weight effect was also significant. PNIPAM L-4 with a molecular weight of about $1 / 10$ of $L-6 B$, had $t^{*}$ values about $1 / 10$ of those for $L-6 B$ at the same concentration. Also, L-4 displayed less temperature sensitivity than did L-6B. 
The corresponding meso-equilibrium $\left(\gamma_{m}\right)$ surface tension values are summarized in

Table 2. 3. For concentrations greater than $5 \mathrm{ppm}$ the $\gamma_{m}$ values were independent of concentration, in agreement with the results of Fujishige et al [15].

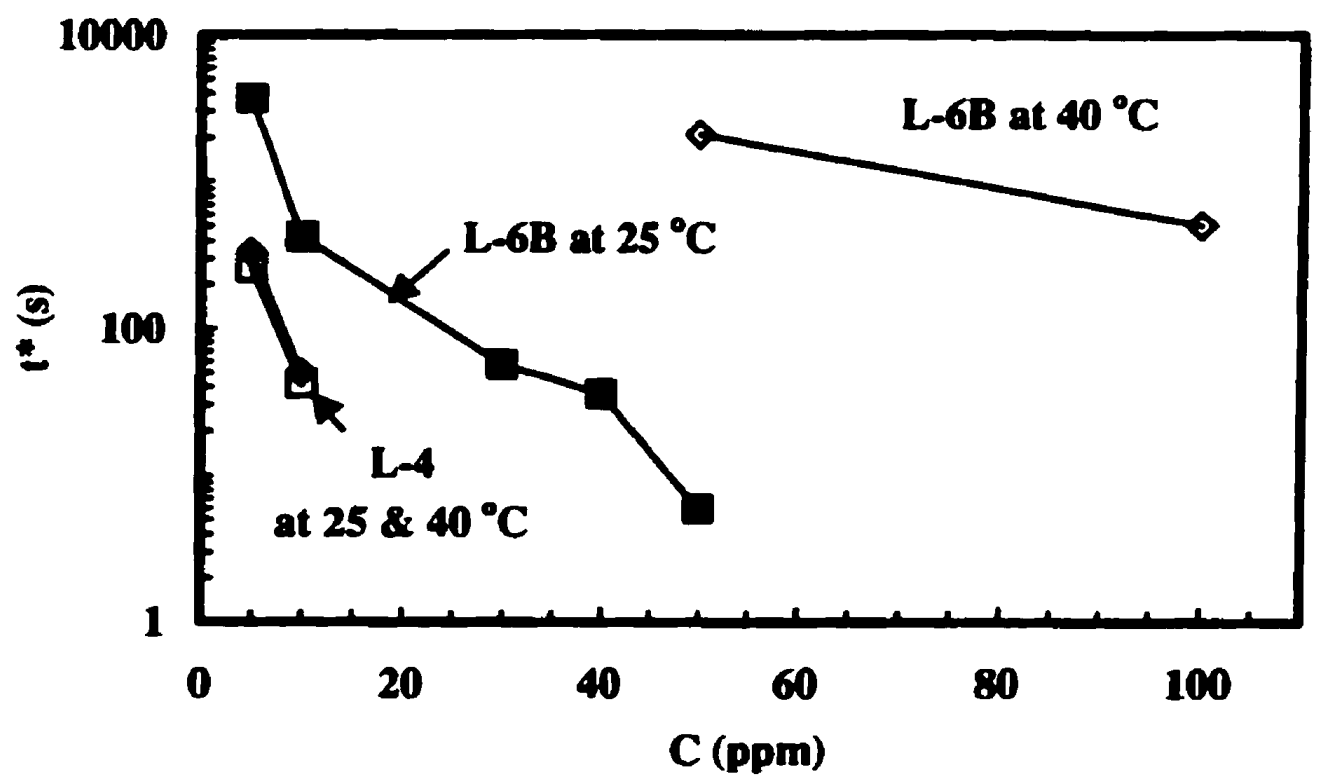

Figure 2.6 as a function of polymer concentration, temperature and molecular weight. 

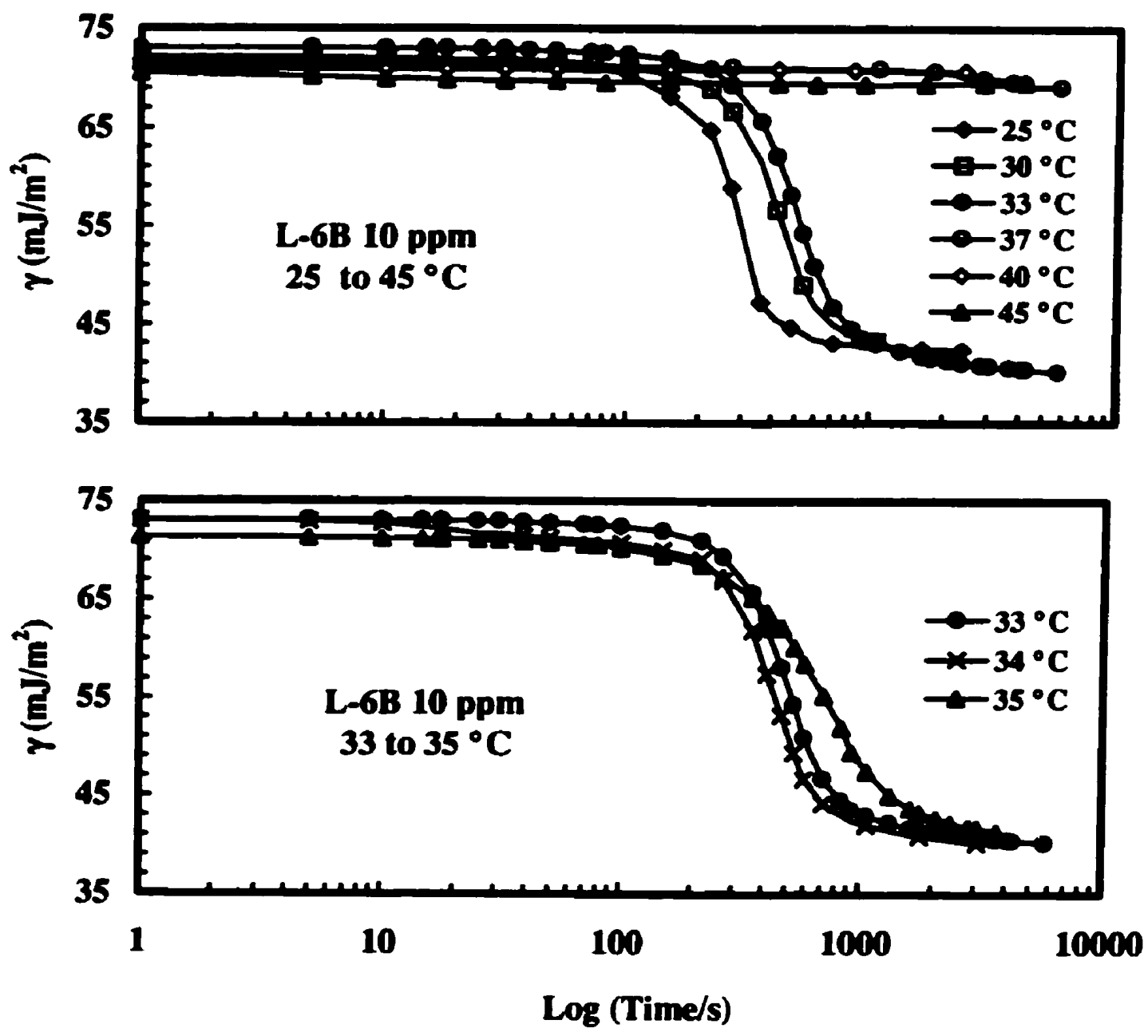

Figure 2.7 The temperature dependence of the dynamic surface tension of PNIPAM (L-6B) 10 ppm aqueous solution. 

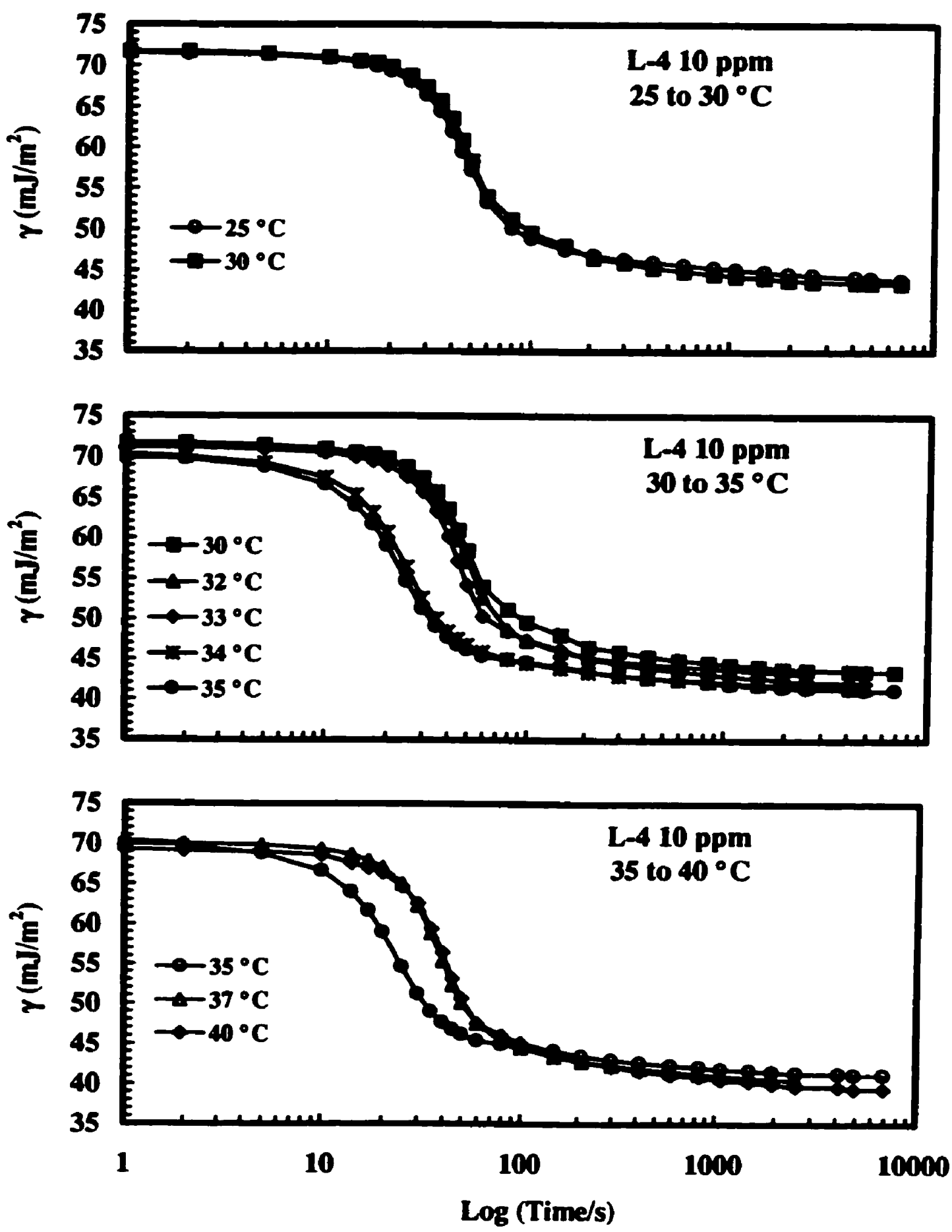

Figure 2.8 The temperature dependence of the dynamic surface tension of PNIPAM (L-4) 10 ppm water solution. 
For experiments involving low polymer concentrations, adsorption at the air/water interface can change the bulk polymer concentration. In a typical experiment, the drop volume was $0.023 \mathrm{ml}$ with a corresponding surface area of $0.36 \mathrm{~cm}^{2}$. Using Kawaguchi's ellipsometry results we estimate that saturation surface coverage corresponds to about $0.8 \mathrm{mg} / \mathrm{m}^{2}$ (see Appendix 2.5). Based on these numbers a mass balance analysis reveals that adsorption reduces the bulk concentration by about $11 \%$ at $10 \mathrm{ppm}$ compared with only a reduction of about 2\% in bulk concentration for the $50 \mathrm{ppm}$ experiments (see Appendix 2.6).

Dynamic surface tensions were recorded for dilute (10 ppm) polymer solutions for temperatures from 25 to $40{ }^{\circ} \mathrm{C}$ and the results are plotted in Figure 2. 7 and Figure 2. 8. The corresponding Hua-Rosen coefficients are given in Table 2. 3. The $t^{*}$ values are plotted as a function of temperature in Figure 2. 9. The L-6B kinetics slowed with increasing temperature whereas L-4 showed similar behavior at the lowest and highest temperatures. $t^{*}$ for both polymers dipped at $34{ }^{\circ} \mathrm{C}$. As before, the higher molecular weight L-6B displayed much slower kinetics than did L-4. Furthermore, after phase separation the very dilute L-6B dispersions did not lower the surface tension in the time scale of the experiment.

The meso-equilibrium surface tensions showed small temperature sensitivity. Figure 2. 10 shows $\gamma_{m}$ as functions of temperature for the $10 \mathrm{ppm}$ solutions. The surface tension of L-4 decreased by about $4 \mathrm{~mJ} / \mathrm{m}^{2}$ when the 
temperature sensitivity is small; the surface tension of water decreases $2.5 \mathrm{~mJ} / \mathrm{m}^{2}$ over the same temperature range.

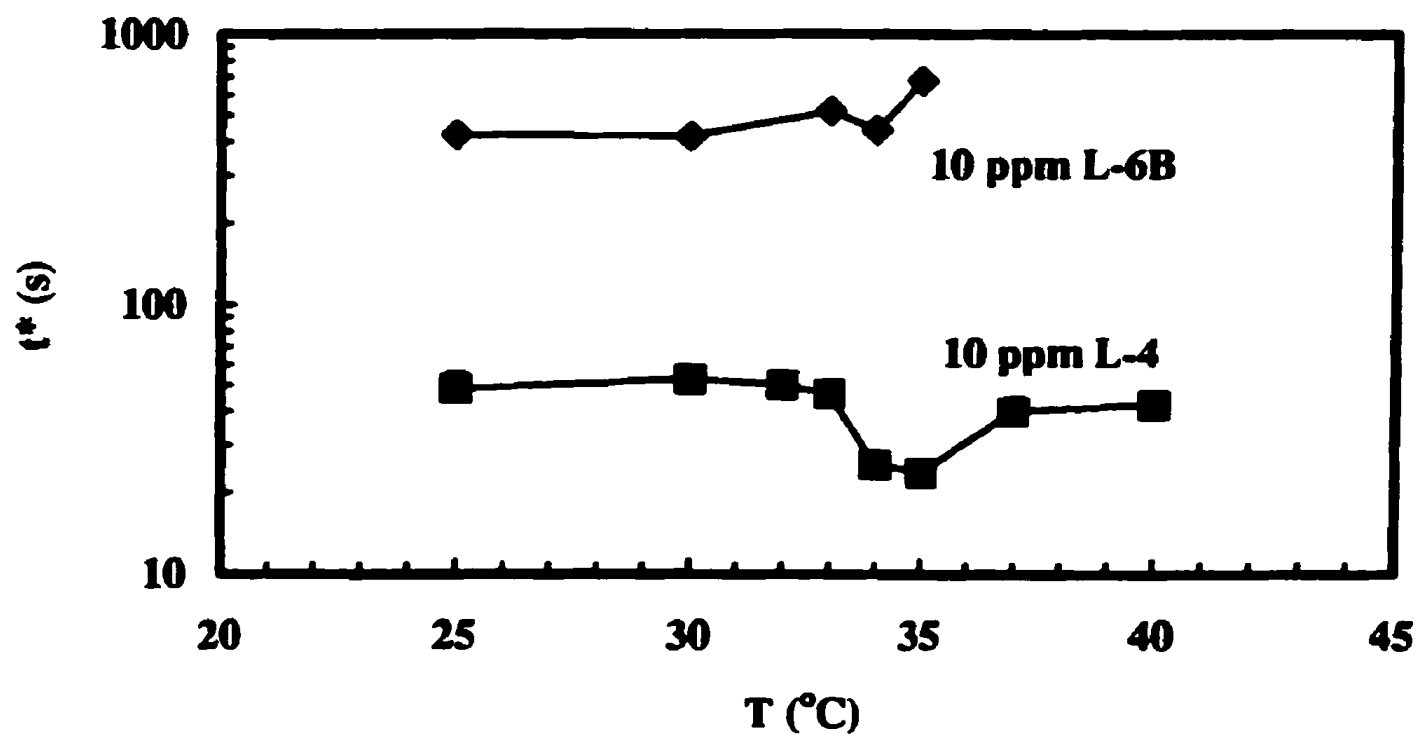

Figure 2.9 t* as a function of temperature for 10 ppm PNIPAM L-6B and L-4.

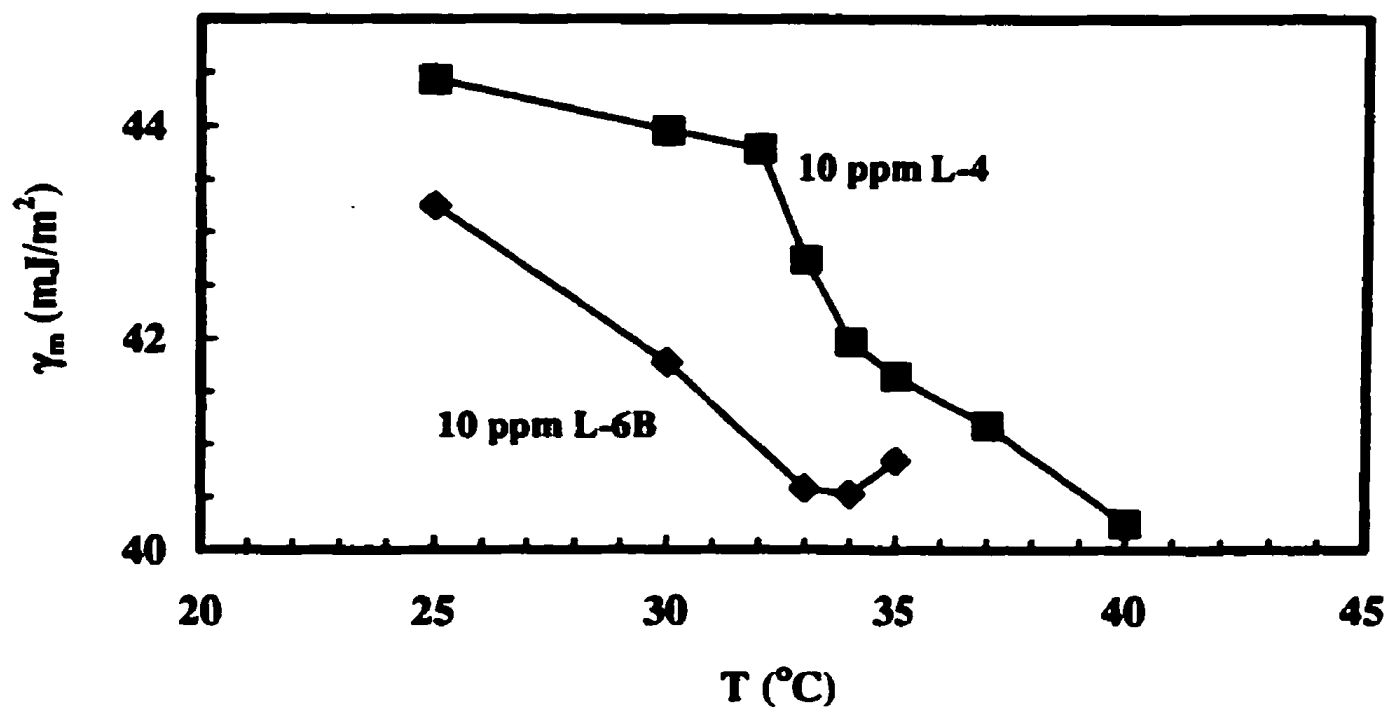

Figure 2. 10 Meso-equilibrium surface tensions as functions of temperature. 


\subsubsection{Oscillating Drop Volume Experiment}

In the oscillating drop volume experiments a pendent drop was formed and polymer solution was pumped into and then out of the drop while recording drop shape to give both surface tension and surface area. The reciprocating pumping caused the drop surface area to vary approximately sinusoidally and surface tension values for 10 and 50 ppm L-6B were recorded as functions of time.

The time-dependent surface tension, $\chi(t)$, and corresponding surface area, $A(t)$, were fit to the following two empirical equations and the results for three different frequencies are summarized in Table 2. 4:

$$
\begin{array}{cc}
A(t)=A_{0}+A_{1} \cos \left(\frac{2 \pi}{P}+\phi\right) & \text { Equation } 2.3 \\
\gamma(t)=\frac{1}{2}\left(1+\cos \left(\frac{2 \pi}{P}+\varphi\right)\right)\left(\gamma_{\max , m}-\gamma_{\min , m}+\frac{\gamma_{s}-\gamma_{\max , m}}{1+\left(\frac{t}{t_{\max }^{*}}\right)^{n}-}-\frac{\gamma_{s}-\gamma_{\min , m}}{1+\left(\frac{t}{t_{\min }^{*}}\right)^{n}-}\right) \\
+\gamma_{\min , m}+\frac{\gamma_{s}-\gamma_{\min , m}}{1+\left(\frac{t}{t_{\min }^{*}}\right)^{n}-}
\end{array}
$$

Equation 2.4 
Table 2. 4 Parameters of the dynamic surface tension of L-6B aqueous solutions in the Oscillation Drop Volume experiment.

\begin{tabular}{|c|ccccc|}
\hline$T\left({ }^{\circ} \mathrm{C}\right)$ & 25 & 25 & 25 & 25 & 40 \\
\hline$C_{b}(\mathrm{ppm})$ & 10 & 10 & 10 & 50 & 50 \\
$P(\mathrm{~s})$ & 24.2 & 33.6 & 60.1 & 33.6 & 33.6 \\
$A_{0}\left(\mathrm{~cm}^{2}\right)$ & 0.3204 & 0.3226 & 0.3139 & 0.2526 & 0.2913 \\
$A_{1}\left(\mathrm{~cm}^{2}\right)$ & 0.0978 & 0.0818 & 0.0507 & 0.0895 & 0.0823 \\
$\phi_{(\mathrm{rad})}$ & -0.0918 & -0.4916 & -0.1335 & -0.1243 & -0.3146 \\
$n_{\min }$ & 3.0202 & 1.4034 & 2.8474 & 0.2945 & 1.6769 \\
$r_{\min }\left(\mathrm{mJ} / \mathrm{m}^{2}\right)$ & 41.51 & 43.42 & 43.53 & 40.79 & 39.72 \\
$t_{\min }^{*}(\mathrm{~s})$ & 91.7 & 38.2 & 122.2 & 0.001 & 133.9 \\
$n_{\max }$ & 2.5246 & 2.2726 & 3.0934 & 1.0828 & 1.7702 \\
$\gamma_{\max }\left(\mathrm{mJ} / \mathrm{m}^{2}\right)$ & 45.00 & 45.22 & 44.02 & 43.38 & 42.60 \\
$t_{\max }^{*}(\mathrm{~s})$ & 616.1 & 369.9 & 309.4 & 2.3 & 1625.6 \\
$\varphi_{(\mathrm{rad})}$ & -0.0104 & 0.0872 & -0.0729 & 0.1602 & -0.2679 \\
$R^{2}$ & 0.8923 & 0.9268 & 0.9908 & 0.8772 & 0.9266 \\
\hline
\end{tabular}

The overall fitting is based on two Hua-Rosen fits (see Appendix 2.4 for example) - one gives the time dependence of the maximum surface tension values $\left(t_{\max }^{*}, n_{\max }\right.$ and $\left.\gamma_{\max , m}\right)$ and the other gives the corresponding minimum surface tension values $\left(t_{\min }^{*}, n_{\operatorname{mix}}\right.$ and $\left.\gamma_{\operatorname{mix}, m}\right)$. Similarly the drop area was fit to a cosine function with two empirical parameters $(P, \phi)$ together with the average drop area, $A_{0}$ and the maximum deviation from the average $A_{l}$. The surface tension fits were good with the lowest $R^{2}$ value of 0.88 . 
The extent of surface area changes is also a variable in these experiments. A surface area expansion factor, $\alpha$, can be defined as

$$
\alpha=\frac{A_{\max }}{A_{\min }}
$$

\section{Equation 2.5}

where $A_{\max }$ is the maximum surface area of the pendent drop and $A_{\min }$ is the corresponding minimum area. As with the static drop volume experiments, the surface tension decreased with time as polymer adsorbed at the interface, however, fluctuations corresponding to the surface area changes were apparent. Comparison with the static drop volume results shows that the approach to mesoequilibrium surface tension was faster with the oscillating drop experiments presumably because of pumping induced convection currents which increased the rate of polymer transport to the air/water interface.

A constant surface tension corresponding to meso-equilibrium was not reached in any of the experiments. Instead, at long times the surface tension oscillated with surface area. Furthermore, the amplitudes of the surface tension oscillations at long times were different for the three curves shown in Figure 2.11 - the fluctuations were the lowest for the bottom curves and largest for the top. For reasons put forward in the discussion section, we believe that these differences reflect the different expansion factors used in the three experiments. Figure 2. 12 shows amplitudes of the surface tension fluctuations at long times as functions of 
the expansion factor. The amplitude increased to nearly $4 \mathrm{~mJ} / \mathrm{m}^{2}$ as the expansion factor approached 2.

Figure 2. 13 compares the results for $50 \mathrm{ppm} \mathrm{L-6B}$ at two temperatures. The low temperature curve very quickly approached steady-state surface tension oscillations between 40.79 and $43.38 \mathrm{~mJ} / \mathrm{m}^{2}$. Above the LCST, the approach to steady-state was much slower. At $50 \mathrm{ppm}$, the $t^{*}$ in the static drop volume experiment was also more than two orders of magnitude greater at $40^{\circ} \mathrm{C}$ than at $25^{\circ} \mathrm{C}$ (see Figure 2. 6) 

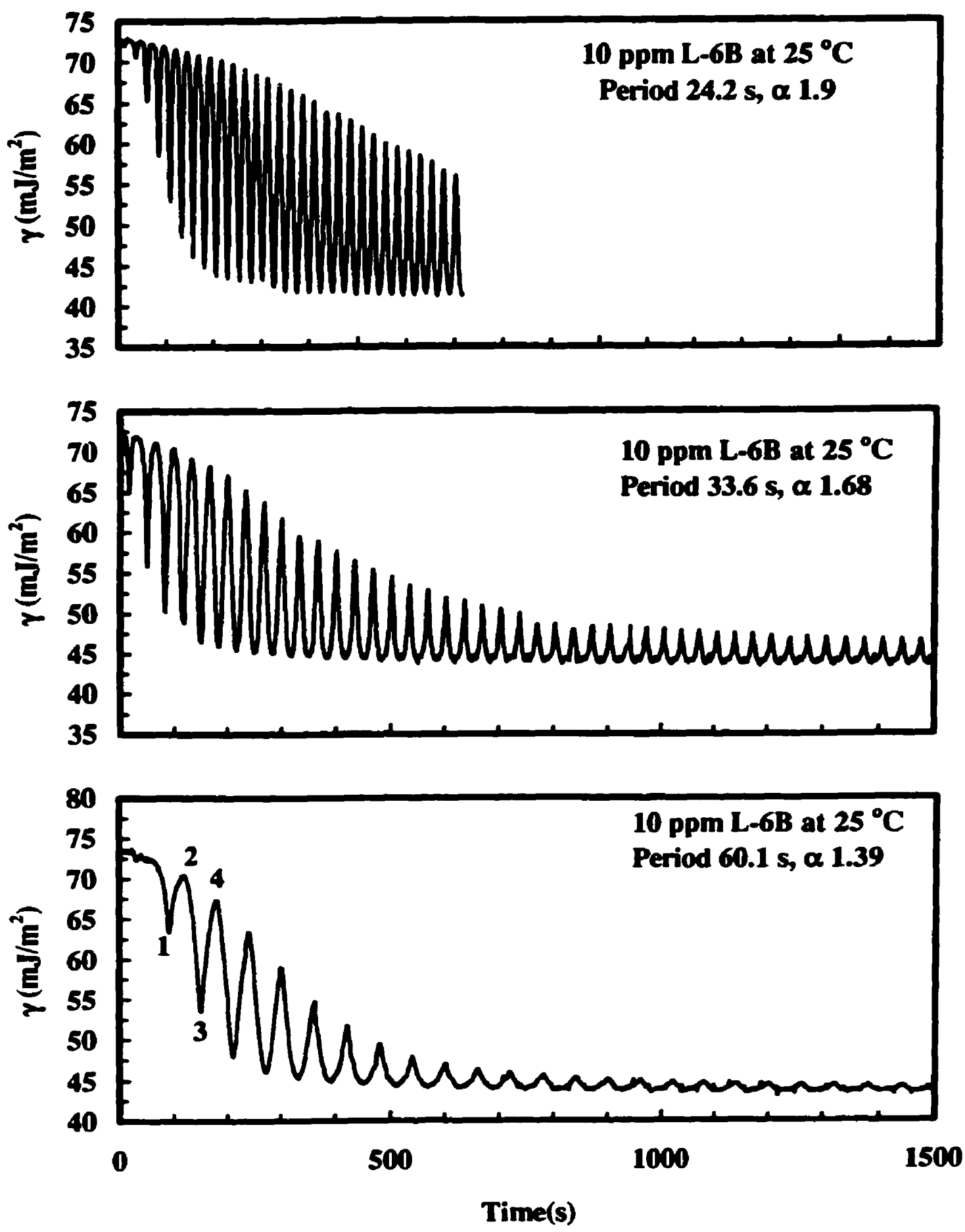

Figure 2. 11 The dynamic surface tension of $10 \mathrm{ppm} \mathrm{L-6B}$ aqueous solution as a function of time at different oscillating frequency periods at $25^{\circ} \mathrm{C}$. 


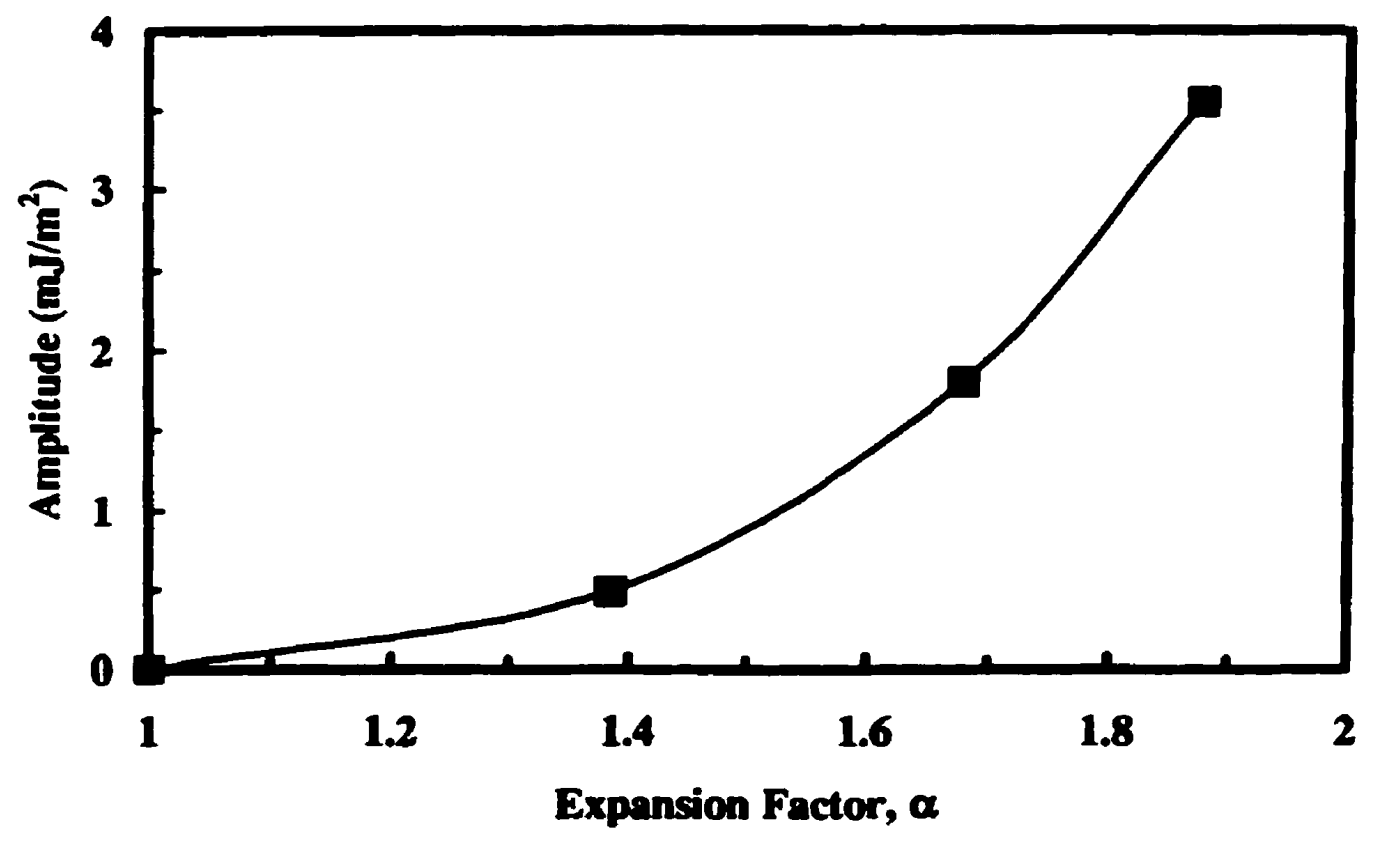

Figure 2. 12 The amplitude of steady-state surface tension fluctuations as a function of the expansion factor $\alpha$ which is the maximum drop area divided by the minimum area. 

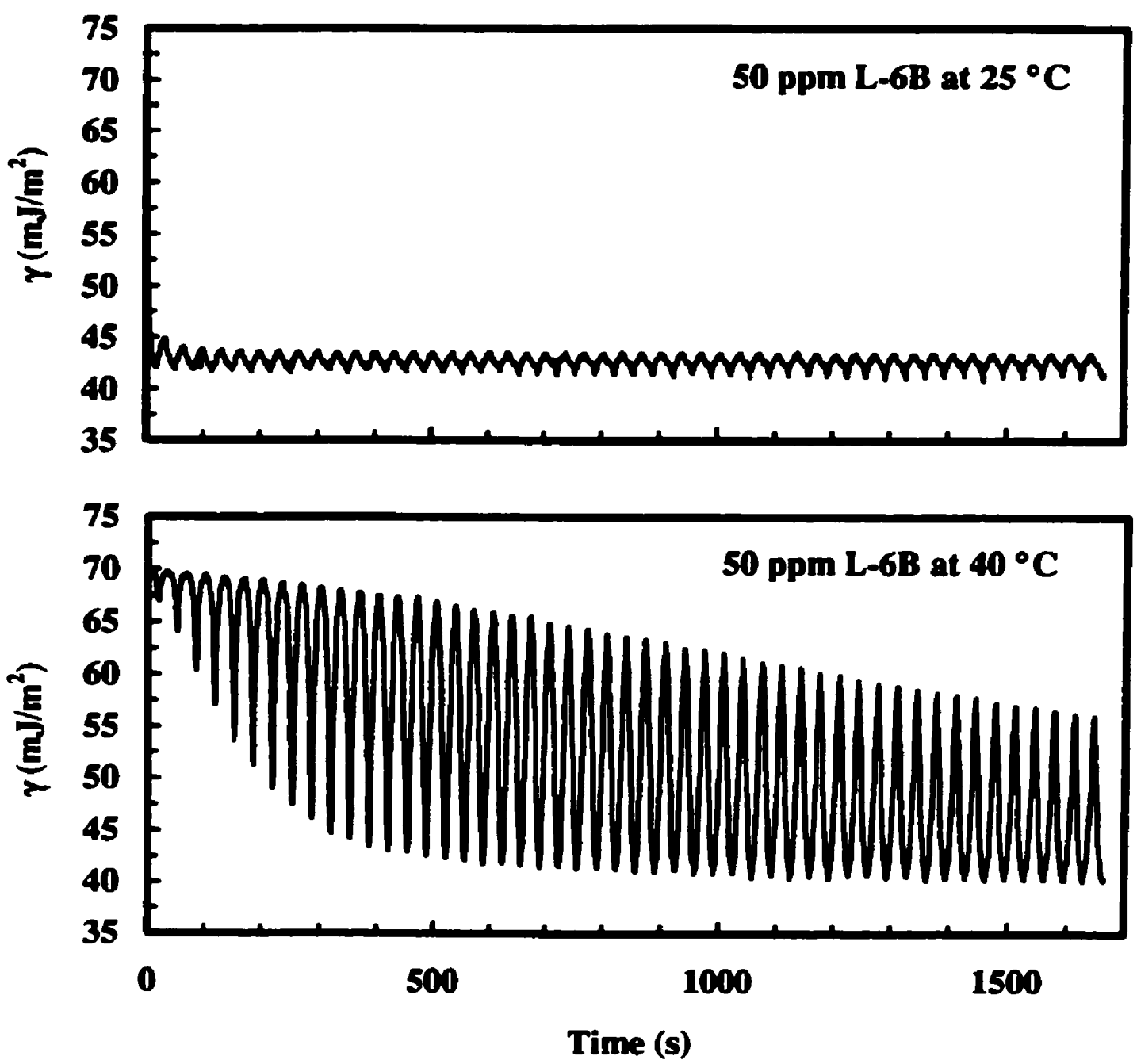

Figure 2. 13 The dynamic surface tension of $50 \mathrm{ppm} \mathrm{L-6B}$ aqueous solution as a function of time with the same oscillating periods $(P=33.55 \mathrm{~s})$ but at different temperatures. 


\subsection{Discussion}

The adsorption of a water-soluble polymer at the air-water interface has been described by a number of authors $[26,27,28,29,30,31]$ and there is general agreement at the simplest level about the probable sequence of events. For dilute solutions, individual polymer chains are believed to diffuse to the interface, attach and re-configure to give an equilibrium conformation. Details of the surface configuration have been probed by modeling, neutron reflection, ellipsometry and surface tension $[32,33]$. There is a body of evidence, both theoretical and experimental suggesting that the apparent equilibrium configuration depends upon the bulk concentration. Layers formed from relatively concentrated solutions are more extended because the available surface area is taken up before chains have a chance to spread out. Indeed, a recently published model specifically accounts for this effect $[34,35]$.

In general, our low temperature dynamic surface tension results are in accord with previously published PNIPAM data as well as the behaviors of other polymer solutions such as aqueous hydroxypropyl cellulose [36]. The time to approach steady-state surface tensions decreased with increasing polymer concentration, suggesting mass transport control of the dynamic surface tension. In addition, the molecular weight had a large influence on the kinetics - high molecular weight PNIPAM reached meso-equilibrium much more slowly than did 
the low molecular weight polymer. On the other hand, the meso-equilibrium surface tensions were not sensitive to molecular weight and our results agreed with those reported by Fujishige et al [15] for the PNIPAM system.

At higher temperatures the system is more complicated and this work is the first extensive report of PNIPAM surface activity above the LCST where the phase separated polymer is present as a stable colloidal dispersion. Longer times were required to obtain steady state surface tension values in comparison with results obtained with true solutions at low temperature. In our experiments the polymer solutions were heated to form the PNIPAM particles before the pendent drops were formed. Therefore, colloidal particles are the only species present to diffuse to the air water interface. However, we do not believe that phase separated particles lower surface tension as particles originally existed in the bulk phase. Instead, the particles must first diffuse from the bulk phase to the air/water interface, then unfold to release polymer chains, which spread on the interface to lower surface tension. Phase separated PNIPAM contains about 30\% water [37, 38] so a phase separated particle contains between about 114 to 1037 polymer chains in the case of L-6B (see Appendix 2.5). Unfolding such particles should take time and it is perhaps surprising that meso-equilibrium surface tensions were observed as quickly as they were.

In previous work, we showed that when an air/water interface, saturated 
with PNIPAM, is formed at low temperature and then heated, there was no discontinuity in surface tension at the LCST of PNIPAM in water [16]. We took this as evidence that PNIPAM adsorbed at the air water interface does not phase separate over the temperature range of 25 to $40^{\circ} \mathrm{C}$. This conclusion is further supported by the results in Figure 2. 10.

The oscillating drop experiments showed two major features. First, at low times the surface tension showed large decaying (in amplitude) surface tension fluctuations in response to the area oscillations. The second major feature was at long times where the surface tension displayed small amplitude, uniform oscillations. These features will now be considered separately.

To understand the initial surface tension oscillations in Figure 2. 11 and Figure 2. 13, one must consider the relationship between surface tension and the concentration of polymer at the interface. Kawaguchi and co-workers [18] reported both the surface pressure and the surface concentration, measured by ellipsometry, as functions of the amount of PNIPAM spread on a Langmuir trough. We have taken their results and constructed plots of surface tension as functions of surface concentration at 16 and $31.3^{\circ} \mathrm{C}$. Linear interpolation was employed to generate the following empirical function to describe the relationship between surface tension and surface concentration at an intermediate temperature of $25^{\circ} \mathrm{C}$. Details of the fitting are given in Appendix 2.4. The surface tension 
versus surface concentration function is plotted in Figure 2. 14.

$$
\gamma_{2 s c}(\Gamma)=42.19+\frac{29.95}{1+2.10 \times 10^{-6} e^{21.7229 \Gamma}}
$$

Equation 2.6

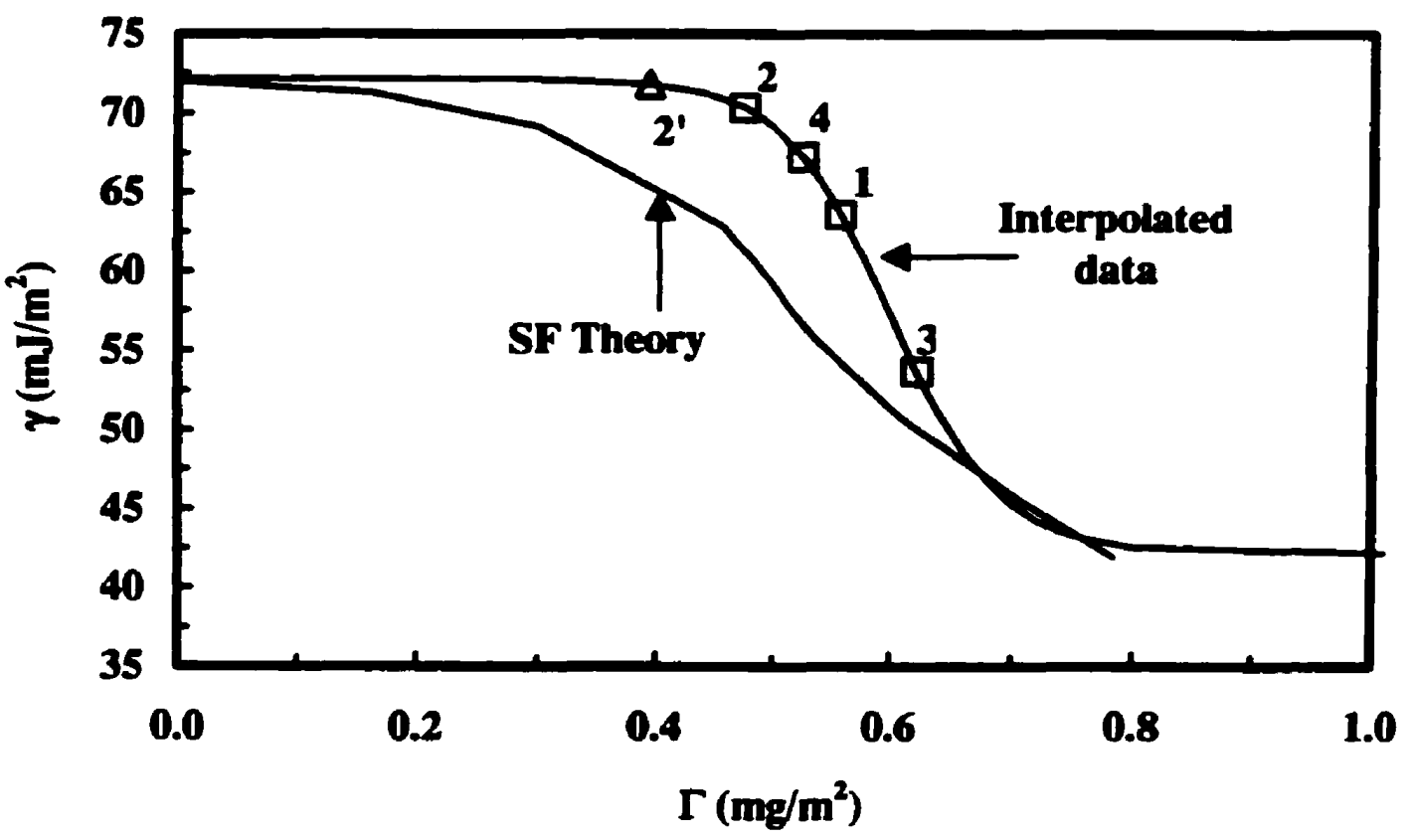

Figure 2.14 Surface tension of aqueous PNIPAM as a function of surface concentration interpolated from Kawaguchi's data [18]. The parameters in the Scheutjens-Fleer calculations are: interaction parameters (kT/site) water/air 6.3. polymer/air 2.435, polymer/water 0.45 ; lattice depth 30 ; chain length 100; and area per site $0.3 \mathrm{~mm}^{2}$.

Also shown in Figure 2. 14 are the results of mean field calculations using Scheutjens-Fleer (SF) theory [32] implemented in the program Goliad, (version 2.1.0.0 kindly provided by Cosgrove and Fleer). The water/surface interaction parameter was chosen to give the surface tension of pure water. Similarly, the 
polymer/surface interaction parameter was chosen to give the minimum surface tension predicted by Equation 2.5. The other program parameters are summarized in the figure caption. SF theory predicted a more gradual decrease in surface tension with increasing surface concentration than did the curve interpolated from Kawaguchi's data.

The lowest curve in Figure 2. 11 has four numbers labeling the peak values for the first two oscillations. These correspond to the open squares labeled by the same numbers in Figure 2. 14. Thus, as the drop area is expanded going from peak 1 to peak 2, the polymer concentration on the interface is decreased giving a higher surface tension. The open triangle labeled by 2' is the calculated position after 1 assuming no polymer adsorption or desorption from the interface using the following equation:

$$
\Gamma_{2^{\prime}}=\Gamma_{1} \frac{A_{1}}{A_{2}}
$$

\section{Equation 2.7}

where $\Gamma$ is the concentration of polymer at the interface and $A$ is the total surface area of the drop (subscript 1 minimum area, 2 maximum area). The corresponding surface tension was used to solve Equation 2.6 for $\Gamma$. Thus the difference, $A_{2}\left(\Gamma_{2}-\Gamma_{2}\right)$ is the mass of additional polymer adsorbed between positions 1 and 2. More detailed modeling and analysis of these results will be presented in a future publication. 
The amplitudes of the steady-state oscillations at long times are small (see Figure 2. 12). For example, if at the minimum drop volume the surface is saturated to give $\Gamma=0.8 \mathrm{mg} / \mathrm{m}^{2}$, then according to Figure 2. 14 , doubling the drop area to give $\Gamma=0.4 \mathrm{mg} / \mathrm{m}^{2}$ should drive the surface tension up to around 72 $\mathrm{mJ} / \mathrm{m}^{2}$ (see Appendix 2.5). Instead, the surface tension swings were only a few $\mathrm{mJ} / \mathrm{m}^{2}$. We propose that the rather small surface tension changes are because dilation of the surface causes loops to be transformed into trains keeping the concentration of adsorbed segments roughly constant. The ability of the surface layer to heal by loop-to-train transitions will be sensitive to the degree of oscillation as shown in Figure 2. 12. Clearly, this mechanism is speculative and requires further verification. 


\subsection{Conclusions}

1. PNIPAM lowers the surface tension of water both below and above the LCST. In the latter case, colloidal polymer particles in the bulk phase diffuse to the interface to unwrap and spread to lower surface tension.

2. The time scale for surface tension lowering decreases with increasing polymer concentration and decreasing molecular weight.

3. The kinetics of surface tension lowering were not very sensitive to temperature for the lower molecular weight $\left(1.31 \times 10^{4}\right)$ PNIPAM in contrast to the behavior of the higher $\left(5.47 \times 10^{5}\right) \mathrm{MW}$ polymer.

4. The meso-equilibrium surface tension showed a slight $\left(\sim 0.3 \mathrm{~mJ} / \mathrm{m}^{2}{ }^{\circ} \mathrm{C}\right)$ decrease with increasing temperature between 25 and $40{ }^{\circ} \mathrm{C}$ with the most dramatic changes occurring around the LCST.

5. Oscillations of the pendent drop surface area induce a small surface tension oscillation at long times. The amplitude is a function of the extent of surface dilation, which reflects the ability of loops to become trains when the surface expands. 


\subsection{Acknowledgment}

We thank Prof. A. Wilhelm Neumann and Dr. Daniel Yu Hing Kwok in the Laboratory of Applied Surface Thermodynamics at the University of Toronto for allowing us to use their facilities and assisting with the surface tension measurements. We thank NSERC for financial support. We also thank Professor Fleer and Professor Cosgrove for kindly supplying the program Goliad, and Professor Cosgrove for useful discussions. Finally we wish Professor Neumann many more years of fun and productivity. 


\subsection{References}

1 Schild, H. G., Prog. Polym. Sci., 17, 163 (1992).

2 Williams, C.; Brochard, F. and Frisch, H. L., Annu. Rev. Phys. Chem., 23, 3445 (1990).

3 Kubota, K.; Fujishige, S. and Ando, I., J. Phys. Chem., 94, 5154 (1990).

4 Yamamoto, I.; Iwasaki K. and Hirotsu, S., J. Phys. Soc. Jpn., 58, 210 (1989).

5 Chan, K.; Pelton, R. and Zhang, Ju, "On the Formation of Colloidally Dispersed Phase-separated Poly(N-isopropylacrylamide)", submitted to Langmuir.

6 Yoshida, R.; Uchida, K.; Kaneko, Y.; Sakai, K.; Kikuchi A.; Sakurai Y. and Okano, T., Nature, 374, 240 (1995).

7 Yoshida, R.; Sakai, K; Okano T. and Sakurai, Y., J. Biomater. Sci. Polymer Edn., 6, 585 (1994).

8 Bae, Y. H.; Okano T. and Kim, S. W., Pharmaceutical Research, 8, 531 (1991).

9 Okano, T.; Bae, Y. H.; Jacobs, H. and Kim. S. W., J. of Controlled Release, 11,255 (1990).

10 Dong, L. -C. and Hoffman, A. S., J. of Controlled Release, 15, 141 (1991).

11 Dong, L. -C. and Hoffman, A. S., J. of Controlled Release, 14, 21 (1990).

12 Stayton, P. S.; Shimoboji, T.; Long, C.; Chilkoti, A.; Chen, G.; Harris, J. M. and. Hoffman, A. S., Nature, 378, 472 (1995).

13 Chan, K.; Pelton, R. and Zhang, Ju, "On the Formation of Colloidally Dispersed Phase-separated Poly(N-isopropylacrylamide)", submitted to Langmuir.

14 Schild, H. G. and Tirrell, D. A., Langmuir, 7665 (1991). 
15 Fujishige, S.; Koiwai, K.; Kubota, K. and Ando, I., Kenkyu Hokolu-seni Kobunshi Zairyo Konkyusho, 167, 47 (1991).

16 Zhang, Ju and Pelton, R., Langmuir, 12, 2611 (1996).

17 Kawaguchi, M.; Hirose, Y and Kato, T., Langmuir, 12, 3523 (1996).

18 Kawaguchi, M.; Saito, W. and Kato, T., Macromolecules, 27, 5882 (1994).

19 Tanahashi, T.; Kawaguchi, M.; Honda T. and Takahashi, A., Macromolecules, 27, 606 (1994).

20 Rotenberg, L.; Boruvka, Y.; Neumann, A. W., J. Colloid Interface Sci., 93, 169 (1983).

21 Kwok, D. Y.; Vollhardt, D.; Miller, R.; Li., D. and Neumann, A. W., Colloids and Surfaces A: Physicochemical and Engineering Aspects, 88, 51 (1994).

22 Kwok, D., Masters Thesis, University of Toronto, 1994.

23 Jasper, J. J., J. Physical and Chemical Reference Data, 1, 948 (1972).

24 Hua, X. Y. and Rosen, M. J., J. Colloid Interface Sci., 124, 652 (1988).

25 Hua, X. Y. and Rosen, M. J., J. Colloid Interface Sci., 141, 180 (1991).

26 S. R. Milner, Philos. Mag., 13, 96 (1907).

27 Langmuir, I. and Schaefer, V. J., J. Am. Chem. Soc., 59, 2400 ( 1937).

28 Cumper, C. W. N. and Alexander, A. E., Rev. Pure Appl. Chem., 1, 121 (1951).

29 MacRitchie, F. and Alexander, A. E., J. Colloid Sci., 18, 453 (1963).

30 Tripp, B. C.; Magda J. J. and Andrade, J. D., J. Colloid Interface Sci. 173, 16 (1995).

31 Graham, A. D. E. and Phillips, M. C. J. Colloid Interface Sci., 70, 403 (1979.

32 Fleer, G. J.; Cohen Stuart, M. A.; J. M. H. M. Scheutjens; Cosgrove, T. and Vincent, B., Polymers at the Interfaces, Chapman \& Hall, first edition, 1993. 
33 Cohen Stuart, M. A. and Fleer, G. J., Annu. Rev. Mater. Sci., 26, 463 (1996).

34 Cosgrove, T.; Prestidge, C. A.; King, S. M. and Vincent, B., Langmuir, 8, 2206 (1992).

35 Cosgrove, T.; Prestidge, C. A. and Vincent, B., J. Chem. Soc. Faraday Trans., 86, 1377 (1990).

36 Chang, S. A. and Gray, D.G., J. Colloid Interface Sci., 67, 255 (1978).

37 Osada, Y. and Ross-Murphy, S.B. Scientific America, May, 82 (1993).

38 Dong, L. C. and Hoffman, A. S., J. Controlled Release, 13, 21 (1990). 


\section{Appendix 2.1 Calculation of $\mathbf{R}_{\mathrm{g}}$ and $\mathbf{C}_{\mathrm{o}}$}

This calculation is to approximate the radius of gyration $\left(\mathbf{R}_{g}\right)$ and overlapping concentration $\left(C_{0}\right)$ of $L-4$ and L-6B poly(N-isopropylacrylamide) (PNIPAM) homopolymers in water solutions.

According to Kubota et al.'s Table I [1], at $20^{\circ} \mathrm{C}$, for PNIPAM in water:

$$
\begin{array}{ll}
M_{\mathrm{w} 1}:=1.63 \cdot 10^{6} \cdot \frac{\mathrm{gm}}{\mathrm{mole}} & R_{\mathrm{g} 1}:=51.0 \mathrm{~nm} \\
M_{\mathrm{w} 2}:=4.10 \cdot 10^{6} \cdot \frac{\mathrm{gm}}{\mathrm{mole}} & R_{\mathrm{g} 2}:=88.0 \mathrm{~nm}
\end{array}
$$

where $M_{w}$ is the weight average molecular weight of polymer, 1 and 2 indicate Sample 1 and Sample 2 respectively.

Assume: $\quad R_{g}=K \cdot M_{w} v^{v} \quad$ ( $K$ and $v$ are constants)

Then,

$$
v:=\frac{\log \left(\frac{51}{88}\right)}{\log \left(\frac{1.63}{4.1}\right)} \quad v=0.59
$$

According to Flory-Krigbaum's theory for dilute polymer solution,

$$
R_{g}=\left\langle R_{g}^{2}\right\rangle^{0.5} \text { and } \quad R_{g} \text { is proportional to } M_{w}^{0.6}
$$

That is, the approximation of $v$ is in good agreement with Flory-Krigbaum's theory and

$$
R_{g}=K \cdot M_{w}{ }^{0.59} \quad \text { Equation A2.1 }
$$

is used to approximate $R_{g}$ 
10 calculate the overlapping concentration of polymer chains in water, the tollo relationship is used [1]:

$$
C_{0}=\frac{3 \cdot M_{w}}{4 \cdot \pi \cdot N_{a v} \cdot R_{g}^{3}}
$$

where

$$
N_{\text {av }} \text { is the Avogadro's number and } N_{\text {av }}:=6.0221367 \cdot \frac{10^{23}}{\text { mole }}
$$

For $L-4$, according to GPC measuremen $M_{w}:=1.31 \cdot 10^{5} \cdot \frac{\mathrm{gm}}{\text { mole }}$

$$
\begin{array}{ll}
R_{\mathrm{g}}:=51.0 \cdot \mathrm{nm} \cdot\left(\frac{1.31 \cdot 10^{5}}{1.63 \cdot 10^{6}}\right)^{.591} & R_{\mathrm{g}}=11.49 \cdot \mathrm{nm} \\
\mathrm{C}_{0}:=\frac{3 \cdot \mathrm{M}_{\mathrm{w}}}{4 \cdot \pi \cdot \mathrm{N}_{\mathrm{av}} \cdot \mathrm{R}_{\mathrm{g}}{ }^{3}} & \mathrm{C}_{0}=34.2 \cdot \mathrm{ppm}
\end{array}
$$

For L-6B, according to GPC measurement $M_{w}:=5.47 \cdot 10^{5} \frac{\mathrm{gm}}{\text { mole }}$

$$
\begin{array}{ll}
R_{g}:=51.0 \cdot \mathrm{nm} \cdot\left(\frac{5.47 \cdot 10^{5}}{1.63 \cdot 10^{6}}\right)^{.591} & R_{g}=11.49 \cdot \mathrm{nm} \\
C_{0}:=\frac{3 \cdot M_{w}}{4 \cdot \pi \cdot N_{a v} \cdot R_{g}^{3}} & C_{o}=34.2 \cdot \mathrm{ppm}
\end{array}
$$

\section{Reference:}

1. Kubota, K., Fujishige, S. and Ando, I., Single-Chain Transition of Poly(N-isopropylacrylamide, J. Phys. Chem., 94, 5154 (1990). 


\section{Appendix 2.2 Pendent Drops and Corresponding \\ Surface Tensions, Area and Volume}

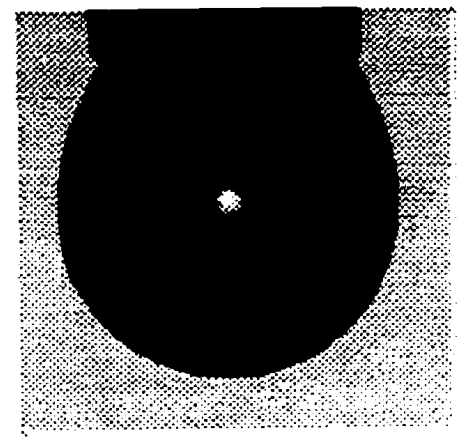

\section{Drop A}

Surface Tension: $61.30 \pm 0.06 \mathrm{~mJ} / \mathrm{m}^{2}$

Area: $0.27638 \pm 0.00002 \mathrm{~cm}^{2}$

Volume: $0.016598 \pm 0.000002 \mathrm{~cm}^{3}$

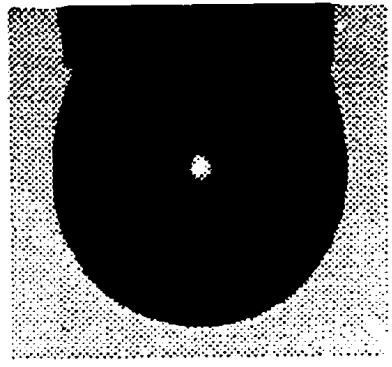

Drop B

Surface Tension: $39.68 \pm 0.03 \mathrm{~mJ} / \mathrm{m}^{2}$

Area: $0.23736 \pm 0.00002 \mathrm{~cm}^{2}$

Volume: $0.013471 \pm 0.000002 \mathrm{~cm}^{3}$

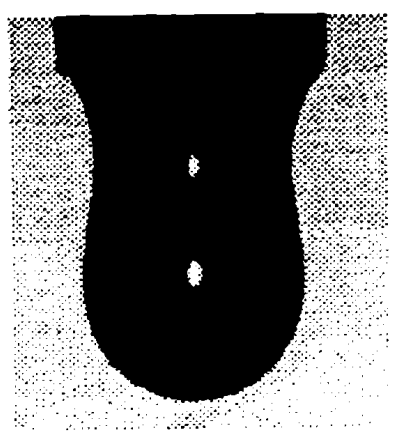

Drop C

Surface Tension: $17.18 \pm 0.03 \mathrm{~mJ} / \mathrm{m}^{2}$

Area: $0.22991 \pm 0.00002 \mathrm{~cm}^{2}$

Volume: $0.010483 \pm 0.000001 \mathrm{~cm}^{3}$ 


\section{Appendix 2.3 Surface Tension of Water as a Function of Temperature}

The following surface tension of pure water is according to Jasper (Joseph J. Jasper, J. Phys. Chem. Ref. Data, 1, No. 4, 948 (1972).).

\begin{tabular}{rr} 
T(C) & \multicolumn{1}{r}{$\gamma_{0}\left(\mathrm{~m} / \mathrm{m}^{2}\right)$} \\
10 & 74.36 \\
15 & 73.62 \\
20 & 72.88 \\
25 & 72.14 \\
30 & 71.4 \\
35 & 70.66 \\
40 & 69.92 \\
45 & 69.18 \\
50 & 68.45 \\
60 & 66.97 \\
70 & 65.49 \\
80 & 64.01 \\
90 & 62.54 \\
100 & 61.06
\end{tabular}

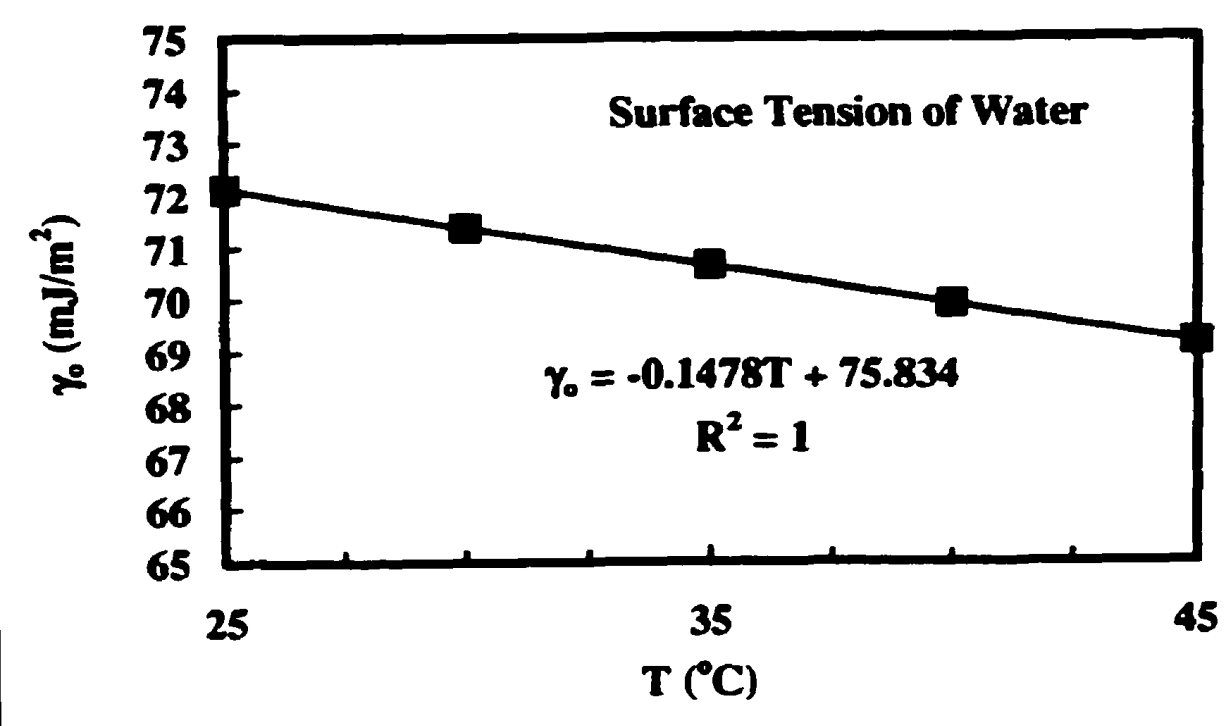


According to the equation obtained through fitting the above data to a straight line, surface tensions of water at the following temperatures are calculated, which are used in calculations in this thesis.

T ( $\left.{ }^{\circ} \mathrm{C}\right) \quad \gamma_{0}\left(\mathrm{~m} \mathrm{~J} / \mathrm{m}^{2}\right)$

$16 \quad 73.4692$

$31.3 \quad 71.20786$

$30 \quad 71.4$

3271.1044

3370.9566

$34 \quad 70.8088$

$35 \quad 70.661$

$37 \quad 70.3654$

$40 \quad 69.922$ 


\section{Appendix 2.4 An Example of Fitting the Dynamic Surface Tension Data to Hua-Rosen Equation}

The objective of this spreadsheet is to fit the dynamic surface tension data at $25^{\circ} \mathrm{C}$ of $50 \mathrm{ppm}$ L-6B aqueous solution to Hua-Rosen Equation and minimize the error in curve fitting by maximize the $R^{2}$ value, using the solver in Microson Excel 97. Refer to Tables 2.2, 2.3 and 2.4.

The Hua-Rosen Equation:

$$
\log \frac{\gamma_{0}-\gamma(t)}{\gamma(t)-\gamma_{0}}=n \operatorname{bg} \frac{t}{t^{*}}
$$

Exptl.

$\mathbf{t}_{\mathbf{i}} \boldsymbol{\gamma}_{\mathbf{i}}$

s $\mathbf{m J} / \mathbf{m}^{2}$

$171.6783 \mathrm{n}$

$270.1703 t^{*}=$

$368.3348 \gamma_{m}=$

465.65

$561.9841 \gamma_{0}=$

$658.0748 \operatorname{sum}(i)=$

754.2109 SSE $=\operatorname{sum}\left(\left(\boldsymbol{\gamma}_{i}-\gamma_{\boldsymbol{l}}\right)^{2}\right)$

851.2673 SST $=\operatorname{sum}\left(\gamma_{1}^{2}\right)-(\operatorname{sum}(\gamma))^{2} / \operatorname{sum}(i)$

$949.2001 R^{2}=1-S S E / S S T$

$10 \quad 47.7404$

1146.748 SSE

$1246.1322 \operatorname{sum}\left(\gamma_{1}^{2}\right)$

$1345.5031 \operatorname{sum}\left(\gamma_{j}\right)$

14 45.4551 SST

1545.1816

$16 \quad 45.021 R^{2}=1-S S E / S S T \quad 0.998476$

1744.6689

$1844.6148 R^{2}$ value is maximized by

1944.306 changing the values of $n, t^{*}$ and

$2044.2717 \gamma_{m}$. This is done by :
At $25^{\circ} \mathrm{C}$, the fitted $\gamma_{s}$ is:

$$
\gamma_{i, f}(t)=\gamma_{m}+\frac{72.14-\gamma_{m}}{1+\left(\frac{t_{i}}{t^{*}}\right)^{n}}
$$

Fitted

$\gamma_{s} \quad\left(\gamma_{-}-\gamma_{h}\right)^{2} \quad \gamma_{s}^{2}$ $\mathrm{mJ} / \mathrm{m}^{2} \quad\left(\mathrm{~mJ} / \mathrm{m}^{2}\right)^{2}\left(\mathrm{~mJ} / \mathrm{m}^{2}\right)^{2}$ $\begin{array}{lllll}2.994741 & 72.0031 & 0.105479 & 5137.78\end{array}$ $\begin{array}{llllll}5.969954 \mathrm{~s} & 71.0834 & 0.833688 & 4923.87\end{array}$

$\begin{array}{llllll}43.14185 & \mathrm{~mJ} / \mathrm{m}^{2} \quad 68.8641 & 0.280152 & 4669.64\end{array}$ $\begin{array}{llll}65.4237 & 0.051227 \quad 4309.92\end{array}$

$\begin{array}{lll}61.4023 & 0.338531 & 3842.03\end{array}$

$\begin{array}{lll}57.5319 & 0.294701 & 3372.68\end{array}$

$\begin{array}{lll}54.2492 & 0.001469 & 2938.82\end{array}$

$\begin{array}{lll}51.6641 & 0.157455 & 2628.34\end{array}$

$\begin{array}{lll}49.7043 & 0.254181 & 2420.65\end{array}$

$\begin{array}{lll}48.2407 & 0.250344 & 2279.15\end{array}$

$\begin{array}{lll}47.1496 & 0.161307 & 2185.38\end{array}$

$\begin{array}{llll}46.3314 & 0.039679 & 2128.18\end{array}$

$\begin{array}{llll}45.7118 & 0.043565 & 2070.53\end{array}$

$\begin{array}{llll}45.2373 & 0.047446 & 2066.17\end{array}$

$44.86940 .097448 \quad 2041.38$

$\begin{array}{llll}44.5809 & 0.193701 & 2026.89\end{array}$

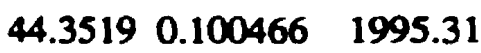

$44.1683 \quad 0.199364 \quad 1990.48$

$\begin{array}{lll}44.0195 & 0.082082 & 1963.02\end{array}$

$\begin{array}{lll}43.8978 & 0.139811 & 1959.98\end{array}$ 


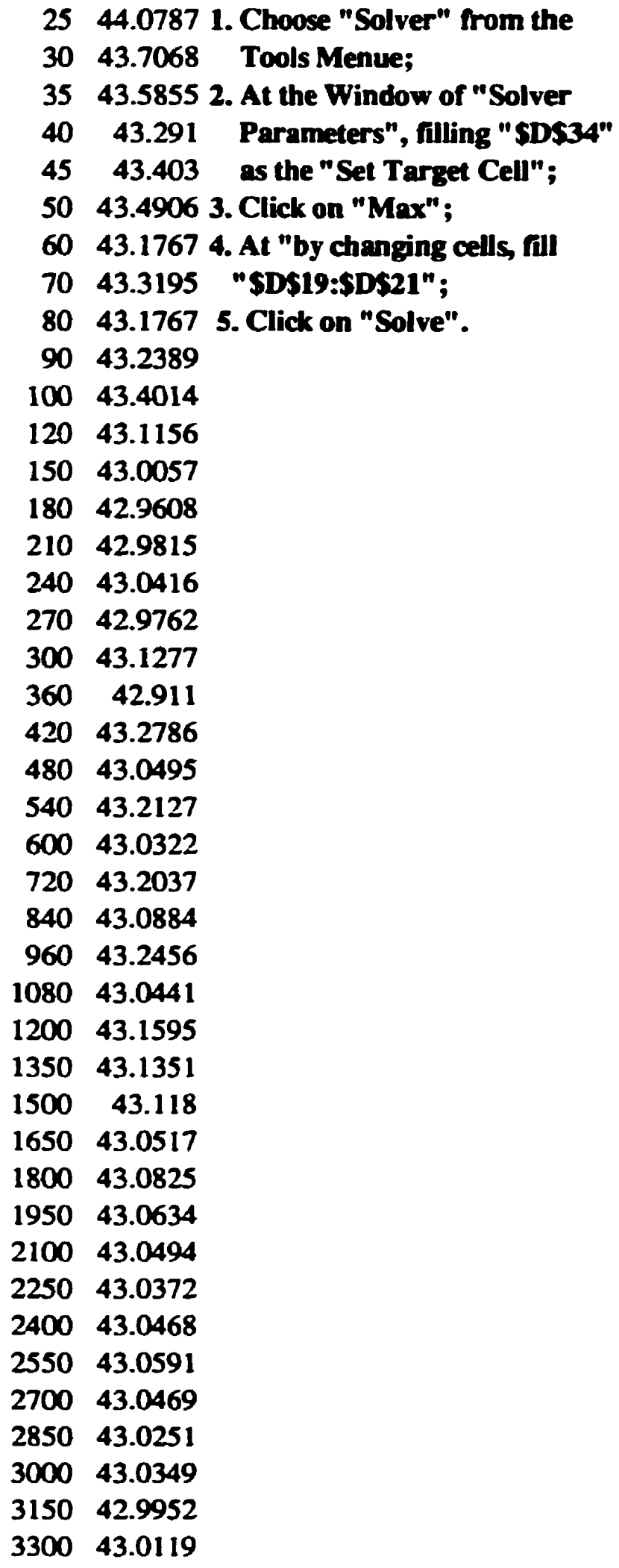

$\begin{array}{lll}43.5343 & 0.296335 & 1942.93\end{array}$ $\begin{array}{llll}43.3705 & 0.113095 & 1910.28\end{array}$

$\begin{array}{llll}43.2864 & 0.089472 & 1899.7\end{array}$

$\begin{array}{lll}43.2389 & 0.002714 & 1874.11\end{array}$

$\begin{array}{lll}43.2101 & 0.037201 & 1883.82\end{array}$

$\begin{array}{lll}43.1917 & 0.089351 & 1891.43\end{array}$

43.1707 3.55E-05 1864.23

$\begin{array}{llll}43.1601 & 0.025419 & 1876.58\end{array}$

$\begin{array}{llll}43.1541 & 0.000512 & 1864.23\end{array}$

$\begin{array}{lll}43.1504 & 0.007826 & 1869.6\end{array}$

$\begin{array}{llll}43.1481 & 0.064153 & 1883.68\end{array}$

$\begin{array}{lll}43.1455 & 0.000893 & 1858.95\end{array}$

$\begin{array}{lll}43.1437 & 0.019048 & 1849.49\end{array}$

$\begin{array}{lll}43.1429 & 0.033172 & 1845.63\end{array}$

$\begin{array}{lll}43.1425 & 0.025932 & 1847.41\end{array}$

$\begin{array}{lll}43.1423 & 0.010142 & 1852.58\end{array}$

$\begin{array}{lll}43.1422 & 0.027548 & 1846.95\end{array}$

$\begin{array}{lll}43.1421 & 0.000207 & 1860\end{array}$

$\begin{array}{lll}43.142 & 0.053356 & 1841.35\end{array}$

$\begin{array}{lll}43.1419 & 0.018676 & 1873.04\end{array}$

$\begin{array}{llll}43.1419 & 0.00854 & 1853.26\end{array}$

$\begin{array}{lll}43.1419 & 0.005013 & 1867.34\end{array}$

$\begin{array}{lll}43.1419 & 0.012031 & 1851.77\end{array}$

$\begin{array}{lll}43.1419 & 0.003823 & 1866.56\end{array}$

$\begin{array}{llll}43.1419 & 0.002859 & 1856.61\end{array}$

$\begin{array}{llll}43.1419 & 0.010762 & 1870.18\end{array}$

$\begin{array}{lll}43.1419 & 0.009557 & 1852.79\end{array}$

$\begin{array}{lll}43.1419 & 0.000311 & 1862.74\end{array}$

$43.1419 \quad 4.57 \mathrm{E}-05 \quad 1860.64$

$\begin{array}{llll}43.1419 & 0.000569 & 1859.16\end{array}$

$\begin{array}{llll}43.1419 & 0.008128 & 1853.45\end{array}$

$\begin{array}{lll}43.1419 & 0.003523 & 1856.1\end{array}$

$\begin{array}{lll}43.1419 & 0.006155 & 1854.46\end{array}$

$\begin{array}{lll}43.1419 & 0.008548 & 1853.25\end{array}$

$\begin{array}{lll}43.1419 & 0.010953 & 1852.2\end{array}$

$\begin{array}{llll}43.1419 & 0.009035 & 1853.03\end{array}$

$\begin{array}{lll}43.1419 & 0.006848 & 1854.09\end{array}$

$\begin{array}{lll}43.1419 & 0.009016 & 1853.04\end{array}$

$\begin{array}{lll}43.1419 & 0.013632 & 1851.16\end{array}$

$43.14190 .011439 \quad 1852$

$\begin{array}{lll}43.1419 & 0.021508 & 1848.59\end{array}$

$\begin{array}{lll}43.1419 & 0.016888 & 1850.02\end{array}$ 


$\begin{array}{lr}3450 & 42.9954 \\ 3600 & 43.0346 \\ 3660 & 43.046 \\ 3720 & 43.0216 \\ 3780 & 43.0215 \\ 3840 & 42.9975 \\ 3900 & 43.041 \\ 3960 & 43.0349\end{array}$

$\begin{array}{lll}43.1419 & 0.021449 & 1848.6\end{array}$

$\begin{array}{lll}43.1419 & 0.011504 & 1851.98\end{array}$

$\begin{array}{llll}43.1419 & 0.009188 & 1852.96\end{array}$

$\begin{array}{llll}43.1419 & 0.014461 & 1850.86\end{array}$

$\begin{array}{llll}43.1419 & 0.014485 & 1850.85\end{array}$

$\begin{array}{llll}43.1419 & 0.020838 & 1848.79\end{array}$

$\begin{array}{lll}43.1419 & 0.010172 & 1852.53\end{array}$

$\begin{array}{lll}43.1419 & 0.011439 \quad 1852\end{array}$

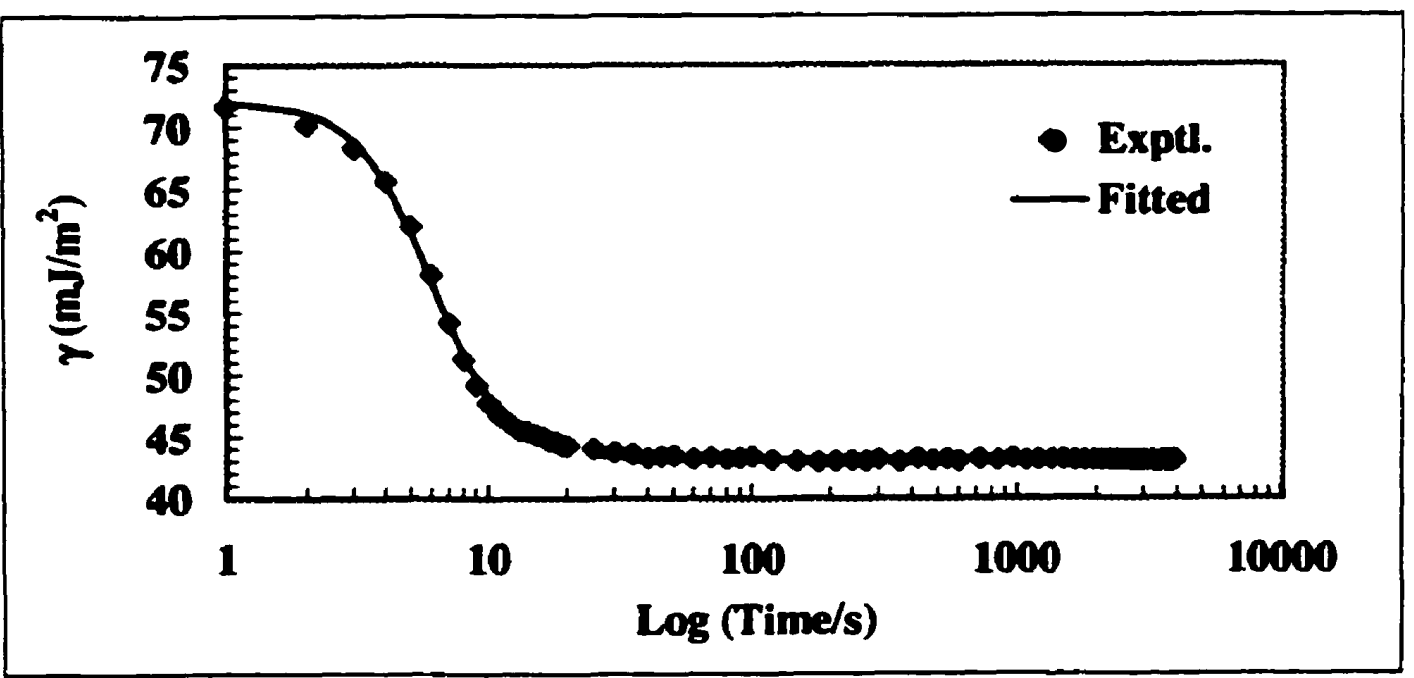




\section{Appendix 2.5 Empirical Relationship between Surface Tension and Surface Concentraion}

All the experimental data in this file were kindly supplied by Dr.

Kawaguchi at Mie Unversity in Japan. Those data had been published in Macromolecules (Kawaguchi, M.; Saito, W. and Kato, T., Macromolecules, 27, 5882 (1994).).

The surface tension of pure water used in this file is sccording to Jasper (Joseph J. Jasper, J. Phys. Chem. Ref. Data, 1, No. 4, 948 (1972).).
$\pi$ : surface pressure. $\mathrm{mJ} / \mathrm{m}^{2}$
C: real spread amount, $\mathrm{mg} / \mathrm{m}^{2}$
$\Gamma$ : ellipsometric surface concentration of PNIPAM, $\mathrm{mg} / \mathrm{m}^{2}$
$\gamma$. surface tension, $\mathrm{mJ} / \mathrm{m}^{2}$

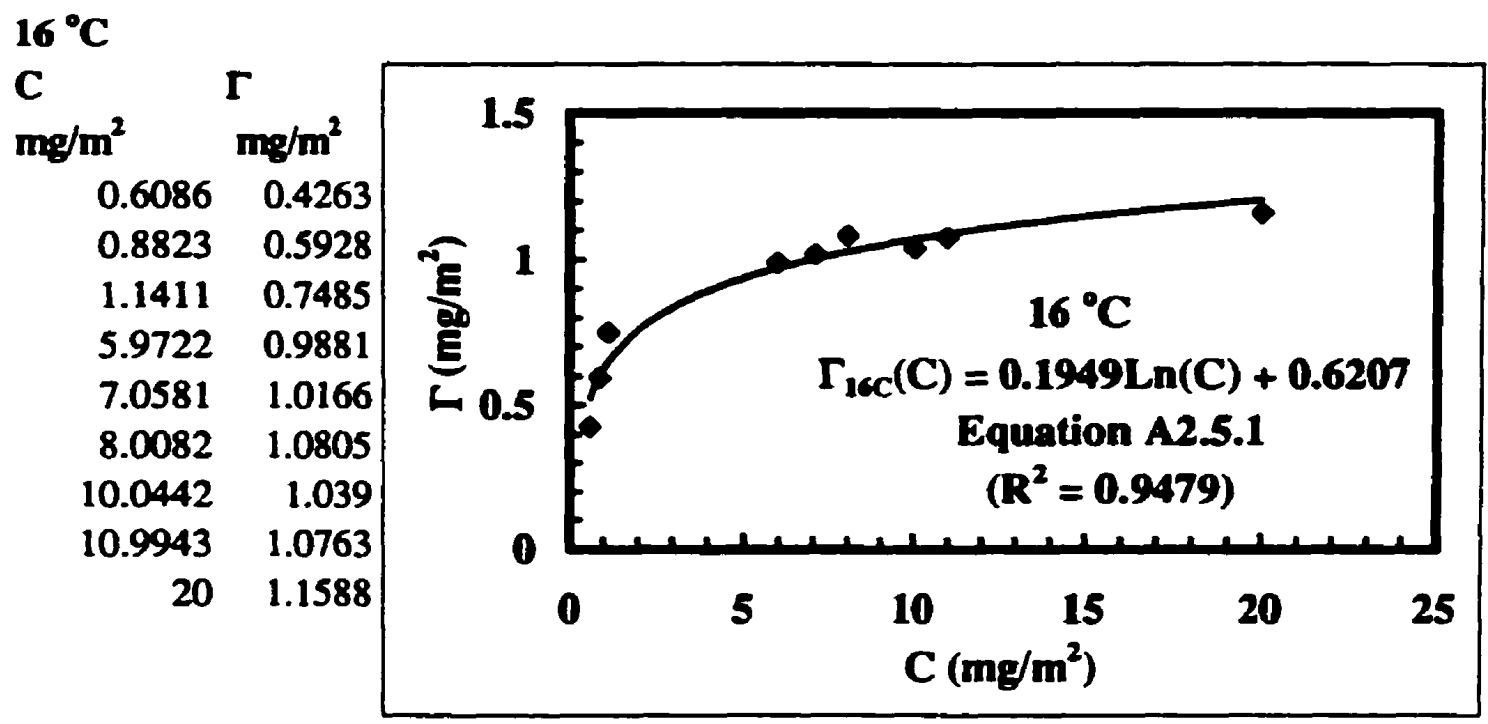




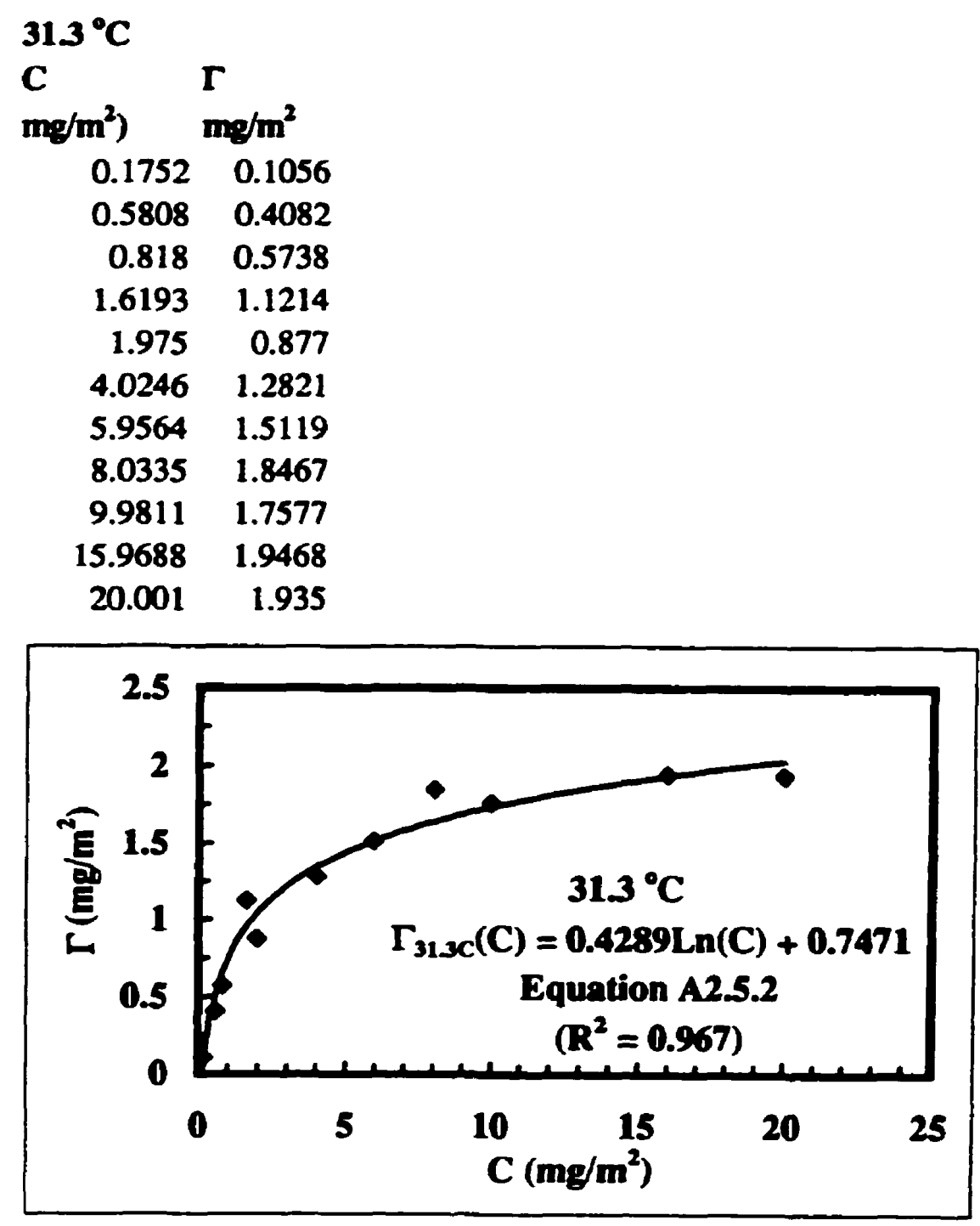


The surface concentration of PNIPAM at the air/water interface at $25^{\circ} \mathrm{C}$ as a function of the real spread amount is interpolated between 16 and $31.3^{\circ} \mathrm{C}$.

$\frac{\Gamma_{16 C}-\Gamma_{2 S C}}{\Gamma_{16 C}-\Gamma_{313 C}}=\frac{16-25}{16-31.3}=0.5882 \Longrightarrow \Gamma_{2 S C}=\Gamma_{16 C}-0.5882\left(\Gamma_{16 C}-\Gamma_{313 C}\right)$

According to Equations A25.1 and A2.52, the surface concentration of PNIPAM at $25^{\circ} \mathrm{C}$ is:

$$
\Gamma_{2 s c}(C)=0.3325 \operatorname{Ln} C+0.695 \quad \text { Equation A2.5.3 }
$$

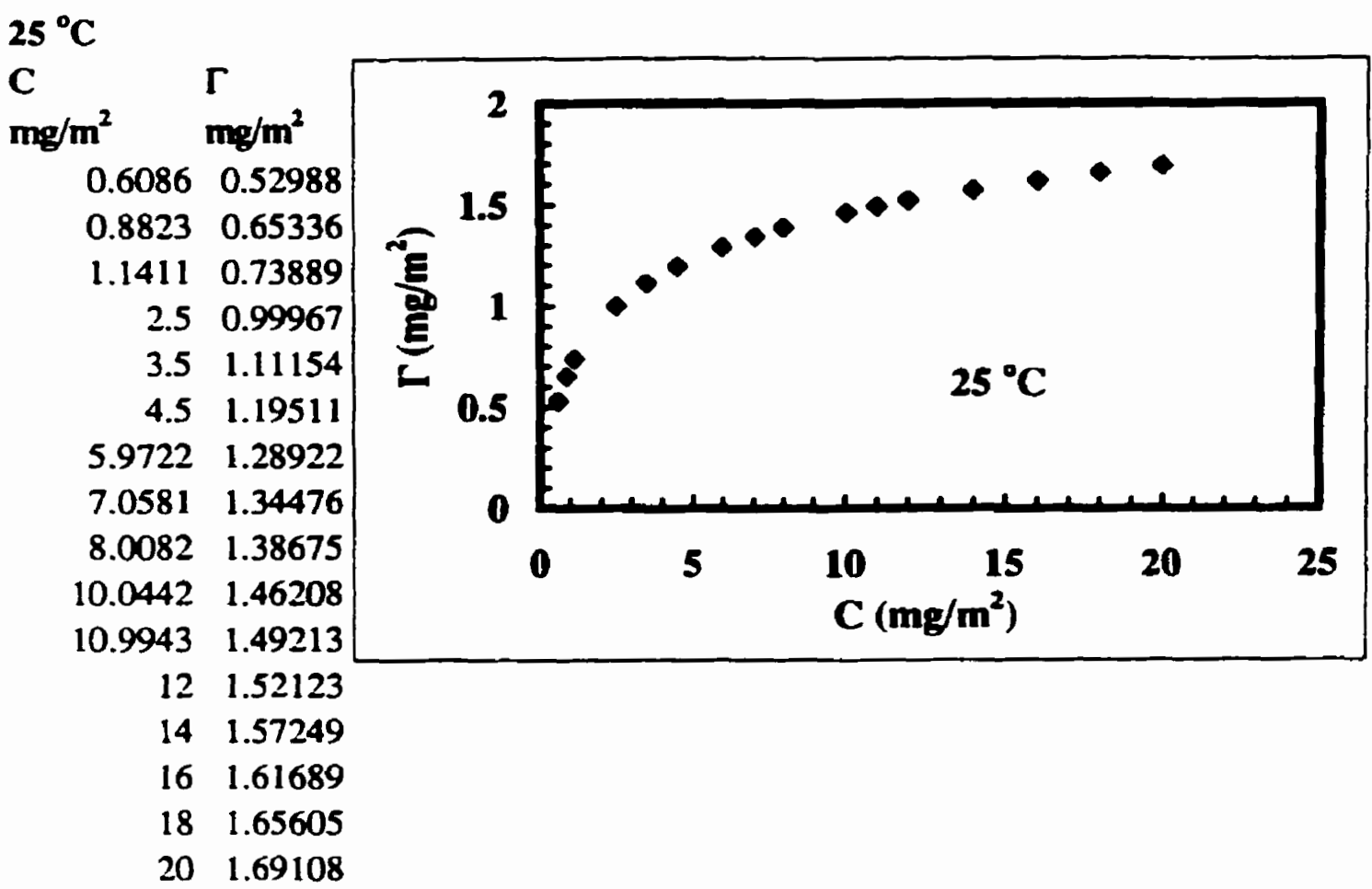


The objective of this spreadsheet is to convert the experimental surface pressure to surface tension and fit the surface tension data as a function of the real spread concentration of PNIPAM at $16^{\circ} \mathrm{C}$ and minimize the error in curve fitting by maximize the $R^{2}$ value, using the Solver in Microson Excel 97.

Assume:

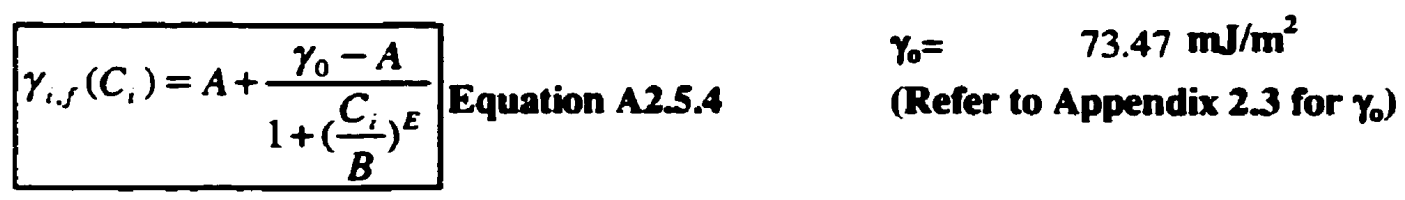

Exptl. Exptl.

$C_{1} \quad \pi_{1} \quad \gamma_{1}=\gamma_{0}-\pi_{4}$

$\mathrm{mg} / \mathrm{m}^{2} \mathrm{~mJ} / \mathrm{m}^{2} \mathrm{~mJ} / \mathrm{m}^{2}$

$0.13280 .040873 .4292 \mathrm{E}=$

$0.1580 .061273 .4088 \mathrm{~B}=$

$0.18470 .081673 .3884 \mathrm{~A}=$

$\begin{array}{lll}0.2086 & 0.102 & 73.368\end{array}$

$0.22590 .122473 .3476 i=1$ to 55

$0.2466 \quad 0.1428 \quad 73.3272$ sum(i)=

0.26670 .163273 .3068 SSE $=\operatorname{sum}\left(\left(\gamma_{1}-\gamma_{\rho}\right)^{2}\right)$

$0.29360 .244873 .2252 \operatorname{SST}=\operatorname{sum}\left(\gamma_{1}^{2}\right)-\left(\operatorname{sum}\left(\gamma_{i}\right)\right)^{2} / \operatorname{sum}(i)$

$0.32240 .326473 .1436 \mathbf{R}^{2}=1-S S E / S S T$

$\begin{array}{llll}0.3358 & 0.3876 & 73.0824\end{array}$

$\begin{array}{llll}0.3488 & 0.3876 & 73.0824 & \text { SSE }\end{array}$

$0.37930 .591672 .8784 \operatorname{sum}\left(\gamma_{1}^{2}\right)$

$\begin{array}{lll}0.3898 & 0.612 & 72.858 \operatorname{sum}\left(x_{1}\right)\end{array}$

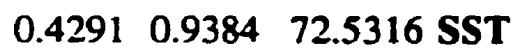

$\begin{array}{lll}0.4514 & 1.0812 & 72.3888\end{array}$

$0.4741 \quad 1.3872 \quad 72.0828 \mathbf{R}^{2}=1-S S E / S S T \quad 0.99902$

$\begin{array}{lll}0.4924 & 1.632 & 71.838\end{array}$

$0.51952 .019671 .4504 \mathbf{R}^{2}$ value is maximized by

0.53342 .427671 .0424 changing the values of $A, B$

0.5693 .121270 .3488 and $E$. This is done by :

0.5954 .141269 .3288 1. Choose "Solver" from the

0.62595 .2632 68.2068 Tools Menue;

0.65666 .874866 .5952 2. At the Window of "Solver

0.70179 .79263 .678 Parameters",filling "SES29"

0.718110 .99662 .4744 as the "Set Target Cell";

0.745312 .50560 .9648 3. Click on "Max";
Fitted

$\gamma_{1, s} \quad\left(\gamma_{1}-\gamma_{1 s}\right)^{2} \quad \gamma_{1 s}^{2}$

$\mathrm{mJ} / \mathrm{m}^{2}\left(\mathrm{~mJ} / \mathrm{m}^{2}\right)^{2}\left(\mathrm{~mJ} / \mathrm{m}^{2}\right)^{2}$

$\begin{array}{lll}73.47 & 0.00166 & 5391.85\end{array}$

$\begin{array}{lll}73.47 & 0.00373 & 5388.85\end{array}$

$\begin{array}{lll}73.47 & 0.00661 & 5385.86\end{array}$

$\begin{array}{lll}73.469 & 0.01023 & 5382.86\end{array}$

$\begin{array}{lll}73.468 & 0.01459 & 5379.87\end{array}$

$\begin{array}{llll}73.467 & 0.01946 & 5376.88\end{array}$

$\begin{array}{llll}73.464 & 0.02464 & 5373.89\end{array}$

$\begin{array}{llll}73.456 & 0.05347 & 5361.93\end{array}$

$\begin{array}{llll}73.441 & 0.08847 & 5349.99\end{array}$

$\begin{array}{lll}73.43 & 0.12062 & 5341.04\end{array}$

$\begin{array}{llll}73.415 & 0.11073 & 5341.04\end{array}$

$\begin{array}{llll}73.362 & 0.23378 & 5311.26\end{array}$

$\begin{array}{llll}73.335 & 0.22773 & 5308.29\end{array}$

$\begin{array}{llll}73.178 & 0.41737 & 5260.83\end{array}$

$\begin{array}{lll}73.031 & 0.41266 & 5240.14\end{array}$

$\begin{array}{llll}72.821 & 0.54544 & 5195.93\end{array}$

$\begin{array}{llll}72.595 & 0.57304 & 5160.7\end{array}$

$\begin{array}{llll}72.14 & 0.47615 & 5105.16\end{array}$

$\begin{array}{llll}71.841 & 0.63733 & 5047.02\end{array}$

$\begin{array}{llll}70.821 & 0.22338 & 4948.95\end{array}$

$\begin{array}{lll}69.812 & 0.23333 & 4806.48\end{array}$

$\begin{array}{llll}68.29 & 0.0069 & 4652.17\end{array}$

$\begin{array}{llll}66.436 & 0.02546 & 4434.92\end{array}$

$\begin{array}{llll}63.215 & 0.21429 & 4054.89\end{array}$

$\begin{array}{llll}61.954 & 0.27116 & 3903.05\end{array}$

$\begin{array}{lll}59.836 & 1.2749 & 3716.71\end{array}$ 
$0.7966 \quad 17.218$ 56.2524 4. At "by changing cells, fill

$0.820719 .31954 .1512 \quad$ "SES14:SES16";

0.880922 .99150 .4792 5. Click on "Solve".

$\begin{array}{lll}0.9438 & 25.602 & 47.868\end{array}$

$\begin{array}{lll}1.0164 & 26.683 & 46.7868\end{array}$

$\begin{array}{lll}1.1105 & 27.581 & 45.8892\end{array}$

$\begin{array}{lll}1.1284 & 27.581 & 45.8892\end{array}$

$\begin{array}{llll}1.2422 & 27.907 & 45.5628\end{array}$

$\begin{array}{lll}1.4028 & 27.989 & 45.4812\end{array}$

$\begin{array}{lll}1.6262 & 28.132 & 45.3384\end{array}$

$\begin{array}{llll}1.8703 & 28.193 & 45.2772\end{array}$

$\begin{array}{llll}1.8807 & 28.172 & 45.2976\end{array}$

$\begin{array}{lll}2.8055 & 28.295 & 45.1752\end{array}$

$3.8966 \quad 28.356 \quad 45.114$

$\begin{array}{llll}4.1793 & 28.336 & 45.1344\end{array}$

$\begin{array}{llll}5.611 & 28.438 & 45.0324\end{array}$

$6.26928 .417 \quad 45.0528$

$\begin{array}{llll}8.3586 & 28.478 & 44.9916\end{array}$

$\begin{array}{llll}10.448 & 28.519 & 44.9508\end{array}$

$\begin{array}{lll}11.3 & 28.54 & 44.9304\end{array}$

$\begin{array}{lll}12.538 & 28.54 & 44.9304\end{array}$

$\begin{array}{lll}14.628 & 28.56 & 44.91\end{array}$

$\begin{array}{llll}14.807 & 28.58 & \mathbf{4 4 . 8 8 9 6}\end{array}$

$\begin{array}{llll}16.717 & 28.58 & 44.8896\end{array}$

$\begin{array}{llll}18.807 & 28.601 & 44.8692\end{array}$

$\begin{array}{llll}20.897 & 28.621 & 44.8488\end{array}$

$\begin{array}{llll}22.986 & 28.642 & 44.8284\end{array}$

$25.076 \quad 28.642 \quad 44.8284$

$27.166 \quad 28.642 \quad 44.8284$

\begin{tabular}{rrr}
\hline 56.036 & 0.04662 & 3164.33 \\
54.447 & 0.08746 & 2932.35 \\
51.206 & 0.52764 & 2548.15 \\
48.894 & 1.05172 & 2291.35 \\
47.254 & 0.21799 & 2189 \\
46.12 & 0.05318 & 2105.82 \\
45.982 & 0.00865 & 2105.82 \\
45.431 & 0.01734 & 2075.97 \\
45.129 & 0.12372 & 2068.54 \\
45.002 & 0.11294 & 2055.57 \\
44.965 & 0.09752 & 2050.02 \\
44.964 & 0.1112 & 2051.87 \\
44.948 & 0.0517 & 2040.8 \\
44.947 & 0.02782 & 2035.27 \\
44.947 & 0.03505 & 2037.11 \\
44.947 & 0.00726 & 2027.92 \\
44.947 & 0.01116 & 2029.75 \\
44.947 & 0.00197 & 2024.24 \\
44.947 & $1.3 E-05$ & 2020.57 \\
44.947 & 0.00028 & 2018.74 \\
44.947 & 0.00028 & 2018.74 \\
44.947 & 0.00138 & 2016.91 \\
44.947 & 0.00331 & 2015.08 \\
44.947 & 0.00331 & 2015.08 \\
44.947 & 0.00608 & 2013.25 \\
44.947 & 0.00968 & 2011.41 \\
44.947 & 0.01411 & 2009.59 \\
44.947 & 0.01411 & 2009.59 \\
44.947 & 0.01411 & 2009.59
\end{tabular}




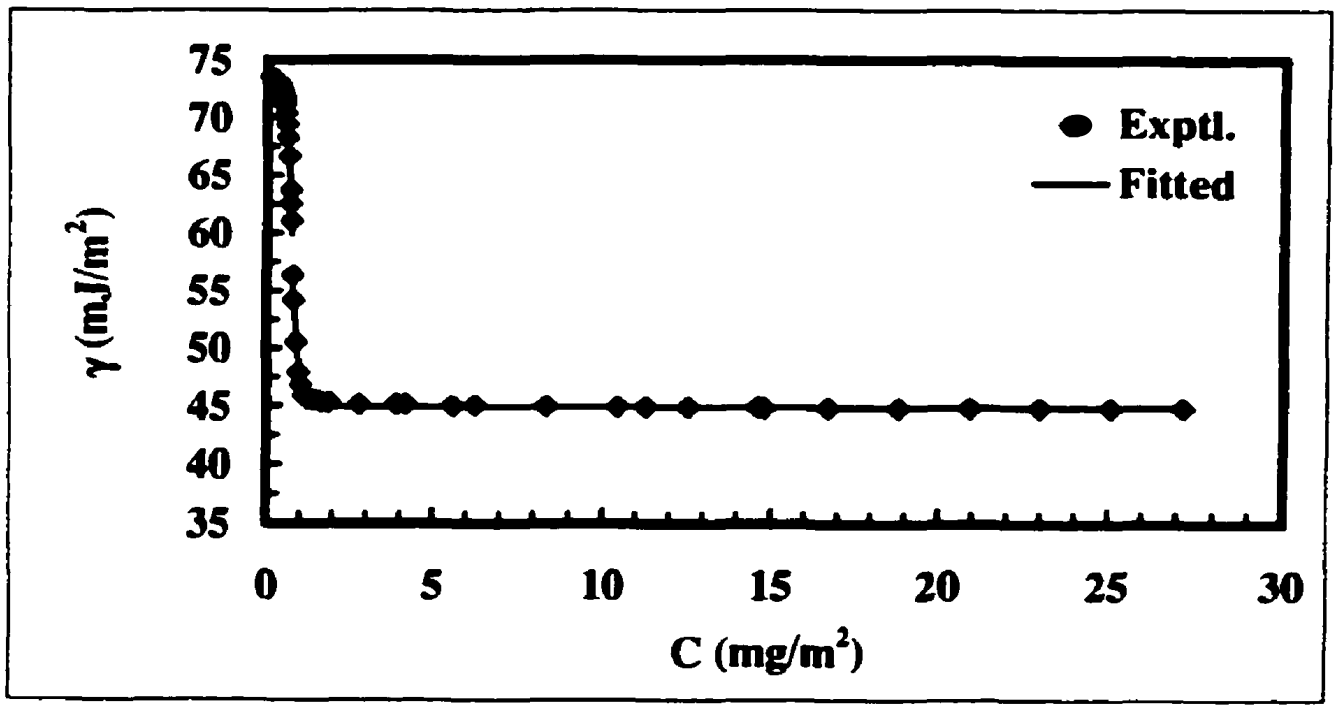

The fitted equation at $16^{\circ} \mathrm{C}$ is

$$
\frac{\gamma_{16 C}(C)=44.9472+\frac{28.5228}{1+\left(\frac{C}{0.7534}\right)^{8.1185}}}{\left(R^{2}=0.9990\right)}
$$

Equation A255 
The objective of this spreadsheet is to convert the experimental surface pressure to surface tension and fit the surface tension data as a function of the real spread concentration of PNIPAM at $31.3^{\circ} \mathrm{C}$ and minimize the error in curve fitting by maximize the $\mathbf{R}^{2}$ value, using the Solver in Microson Excel 97.

Assume:

$$
\gamma_{1, f}\left(C_{1}\right)=A+\frac{\gamma_{0}-A}{1+\left(\frac{C_{i}}{B}\right)^{\varepsilon}}
$$

ExptI. Exptl.

$$
C_{i} \quad \pi_{1} \quad \gamma_{1}=\gamma_{0}-\pi_{1}
$$

$\mathrm{mg} / \mathrm{m}^{2} \mathrm{~mJ} / \mathrm{m}^{2} \mathrm{~mJ} / \mathrm{m}^{2}$

\section{$0.15170 .081671 .1284 \mathrm{E}=$}

$0.17620 .081671 .1284 \mathrm{~B}=$

$0.20860 .124471 .0856 \mathrm{~A}=$

$\begin{array}{lll}0.2466 & 0.204 & 71.006\end{array}$

$0.2517 \quad 0.1836 \quad 71.0264 i=1$ to 56

$0.27690 .2652 \quad 70.9448 \operatorname{sum}(i)=$

$0.3021 \quad 0.3468 \quad 70.8632 \mathrm{SSE}=\operatorname{sum}\left(\left(\gamma_{1}-\gamma_{\mathrm{L} S}\right)^{2}\right)$

0.32240 .469270 .7408 SST $=\operatorname{sum}\left(\gamma_{i}^{2}\right)-\left(\operatorname{sum}\left(\gamma_{1}\right)\right)^{2} / \operatorname{sum}(i)$

$0.32720 .4488 \quad 70.7612 \mathbf{R}^{2}=1-S S E / S S T$

$\begin{array}{lll}0.3524 & 0.612 & 70.598\end{array}$

$\begin{array}{lll}0.3776 & 0.7548 \quad 70.4552 & \text { SSE }\end{array}$

$0.3793 \quad 0.8364 \quad 70.3736 \operatorname{sum}\left(\boldsymbol{\gamma}_{1}^{2}\right)$

$\begin{array}{lll}0.4028 & 0.9588 & 70.2512 \operatorname{sum}\left(\boldsymbol{\gamma}_{1}\right)\end{array}$

$0.4279 \quad 1.2648 \quad 69.9452$ SST

$\begin{array}{lll}0.4531 & 1.5096 & 69.7004\end{array}$

$0.4741 \quad 1.9992 \quad 69.2108 \mathbf{R}^{2}=1$-SSE/SST 0.997646

$\begin{array}{lll}0.5034 & 2.346 \quad 68.864\end{array}$

$\begin{array}{rrr}0.5286 & 2.8968 & 68.3132 \mathbf{R}^{2} \text { value is maximized by } \\ 0.55 & 3.8148 & 67.3952\end{array}$

$0.553 .8148 \quad 67.3952$ changing the values of $A, B$

$0.579 \quad 4.1208$

$0.6041 \quad 5.508$

0.62597 .0584

$0.6545 \quad 7.5888$

$0.6797 \quad 9.7104$

67.0892 and $E$. This is done by :

65.702 1. Choose "Solver" from the

64.1516 Tools Menue;

63.6212 2. At the Window of "Solver

$0.7017 \quad 12.036$

61.4996 Parameters", filling "SES29"

$0.73 \quad 12.607$
59.174 as the "Set Target Cell";

58.6028 3. Click on "Max"; $\gamma_{0}=\quad 71.21 \mathrm{~mJ} / \mathrm{m}^{2}$

(Refer to Appendix 2.3 for $\boldsymbol{\gamma}_{0}$ )
Fitted

$\gamma_{i s} \quad\left(\gamma_{1}-\gamma_{i s}\right)^{2} \quad \gamma_{i s}^{2}$

$\mathrm{mJ} / \mathrm{m}^{2} \quad\left(\mathrm{~mJ} / \mathrm{m}^{2}\right)^{2}\left(\mathrm{~mJ} / \mathrm{m}^{2}\right)^{2}$

$\begin{array}{lll}71.1634 & 0.00123 & 5059.249\end{array}$

$\begin{array}{lll}71.1623 & 0.00115 & 5059.249\end{array}$

$\begin{array}{llll}71.1588 & 0.00536 & 5053.163\end{array}$

$\begin{array}{llll}71.1479 & 0.02013 & 5041.852\end{array}$

$\begin{array}{llll}71.1455 & 0.01418 & 5044.749\end{array}$

$\begin{array}{llll}71.1286 & 0.03379 & 5033.165\end{array}$

$\begin{array}{llll}71.1002 & 0.05618 & 5021.593\end{array}$

$\begin{array}{lll}71.065 & 0.1051 & 5004.261\end{array}$

$\begin{array}{llll}71.0546 & 0.08608 & 5007.147\end{array}$

$\begin{array}{llll}70.9835 & 0.14862 & 4984.078\end{array}$

$\begin{array}{llll}70.8767 & 0.17769 & 4963.935\end{array}$

$\begin{array}{llll}70.8679 & 0.24436 & 4952.444\end{array}$

$\begin{array}{llll}70.7211 & 0.22082 & 4935.231\end{array}$

$\begin{array}{llll}70.5016 & 0.30958 & 4892.331\end{array}$

$\begin{array}{llll}70.1975 & 0.24709 & 4858.146\end{array}$

$\begin{array}{llll}69.8646 & 0.42746 & 4790.135\end{array}$

$\begin{array}{llll}69.2531 & 0.15143 & 4742.25\end{array}$

$\begin{array}{llll}68.5661 & 0.06394 & 4666.693\end{array}$

$\begin{array}{llll}67.8501 & 0.20691 & 4542.113\end{array}$

$\begin{array}{llll}66.6699 & 0.17578 & 4500.961\end{array}$

$\begin{array}{llll}65.4489 & 0.06404 & 4316.753\end{array}$

$\begin{array}{llll}64.2441 & 0.00855 & 4115.428\end{array}$

$\begin{array}{llll}62.4837 & 1.29402 & 4047.657\end{array}$

$\begin{array}{llll}60.7997 & 0.48983 & 3782.201\end{array}$

$\begin{array}{llll}59.2637 & 0.00804 & 3501.562\end{array}$

$\begin{array}{llll}57.2525 & 1.8232 & 3434.288\end{array}$ 


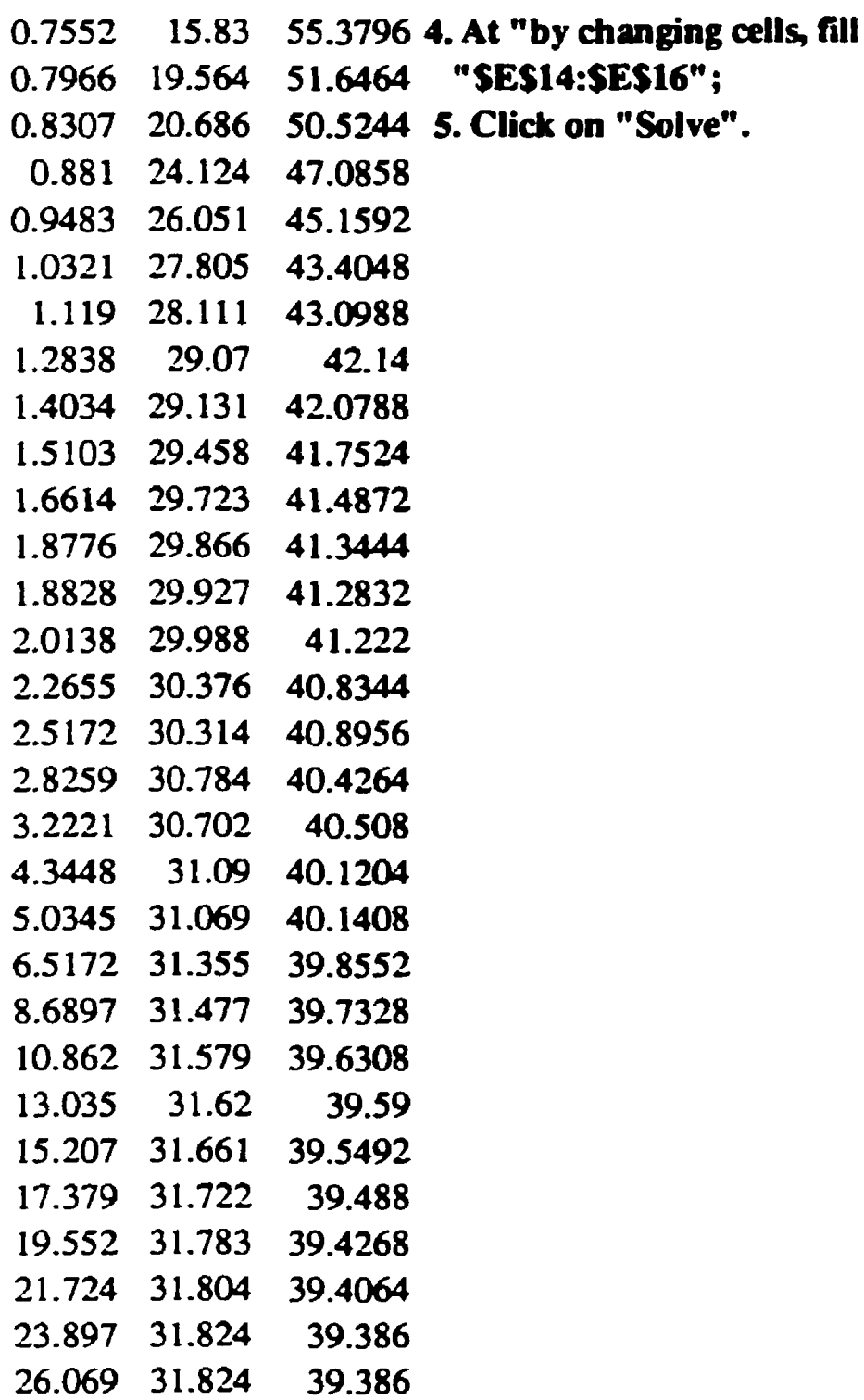

\begin{tabular}{rrr}
\hline 55.4804 & 0.01017 & 3066.9 \\
52.7238 & 1.16086 & 2667.351 \\
\hline 50.681 & 0.02453 & 2552.715 \\
48.1287 & 1.08763 & 2217.073 \\
45.5697 & 0.16854 & 2039.353 \\
43.4953 & 0.00818 & 1883.977 \\
42.2188 & 0.77436 & 1857.507 \\
41.0659 & 1.1536 & 1775.78 \\
40.7084 & 1.87791 & 1770.625 \\
40.5364 & 1.47862 & 1743.263 \\
40.4082 & 1.1643 & 1721.188 \\
40.3281 & 1.0329 & 1709.359 \\
40.3269 & 0.91446 & 1704.303 \\
40.3046 & 0.84168 & 1699.253 \\
40.2833 & 0.30376 & 1667.448 \\
40.2743 & 0.38596 & 1672.45 \\
40.2697 & 0.02456 & 1634.294 \\
40.2674 & 0.05789 & 1640.898 \\
40.266 & 0.0212 & 1609.646 \\
40.2659 & 0.01564 & 1611.284 \\
40.2658 & 0.1686 & 1588.437 \\
40.2658 & 0.28409 & 1578.695 \\
40.2658 & 0.40322 & 1570.6 \\
40.2658 & 0.4567 & 1567.368 \\
40.2658 & 0.51351 & 1564.139 \\
40.2658 & 0.60496 & 1559.302 \\
40.2658 & 0.70391 & 1554.473 \\
40.2658 & 0.73856 & 1552.864 \\
40.2658 & 0.77404 & 1551.257 \\
40.2658 & 0.77404 & 1551.257
\end{tabular}




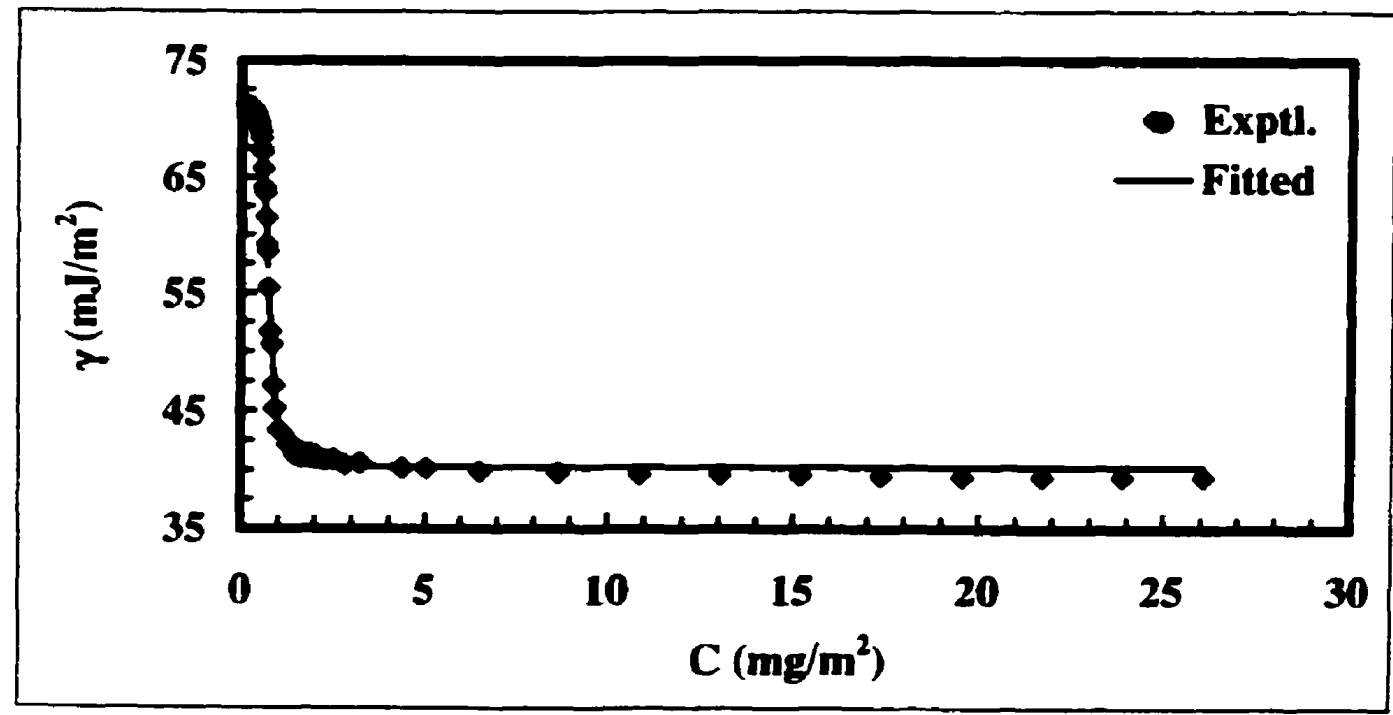

The fitted equation at $31.3^{\circ} \mathrm{C}$ is

$$
\gamma_{31.3 C}(C)=40.2658+\frac{30.9421}{1+\left(\frac{C}{0.7518}\right)^{6.7793}}
$$

Equation A2.5.6

$$
\left(R^{2}=0.9976\right)
$$


The surface tension ( $\gamma$ ) of PNIPAM aqeous solutions at $25^{\circ} \mathrm{C}$ as a function of the real spread amount $(C)$ is interpolated between 16 and $31.3{ }^{\circ} \mathrm{C}$.

$$
\frac{\gamma_{16 C}-\gamma_{2 S C}}{\gamma_{16 C}-\gamma_{31.3 C}}=\frac{16-25}{16-31.3}=0.5882 \Longrightarrow \gamma_{2 S C}=\gamma_{16 C}-0.5882\left(\gamma_{16 C}-\gamma_{31.3 C}\right)
$$

According to Equations A25.5 and A25.6, the surface concentration of PNIPAM at $25^{\circ} \mathrm{C}$ is:

$$
\gamma_{2 s C}(C)=42.1936+\frac{11.7453}{1+\left(\frac{C}{0.7534}\right)^{8.1185}}+\frac{18.2001}{1+\left(\frac{C}{0.7518}\right)^{6.7793}}
$$

Equation A2.5.7

Equation A2.5.6 is further simplified by fitting to Equation A2.5.4

$$
\gamma_{1, f}\left(C_{1}\right)=A+\frac{\gamma_{0}-A}{1+\left(\frac{C_{i}}{B}\right)^{\varepsilon}}
$$

(Refer to Appendix 2.3 for $\gamma_{0}$ )

Exptl. Exptl.

$C_{i} \quad \gamma_{i}$ $\mathrm{mg} / \mathrm{m}^{2} \quad \mathrm{~mJ} / \mathrm{m}^{2}$

$0.1328 \quad 72.1388$

$0.15872 .1385 \mathrm{E}=$

$0.184772 .1375 \mathrm{~B}=$

$0.208672 .1356 \mathrm{~A}=$

0.225972 .1331

$0.246672 .1281 i=1$ to 54

$0.266772 .1202 \operatorname{sum}(i)=$

$0.293672 .1023 \mathrm{SSE}=\operatorname{sum}\left(\left(\gamma_{1}-\gamma_{1}\right)^{2}\right)$

$0.322472 .0685 \operatorname{SST}=\operatorname{sum}\left(\gamma_{1}^{2}\right)-\left(\operatorname{sum}\left(\gamma_{1}\right)\right)^{2} / \operatorname{sum}(i)$

$0.335872 .0453 \mathbf{R}^{2}=1-S S E / S S T$

$0.3488 \quad 72.0168$

0.379371 .9194 SSE

$0.389871 .8732 \operatorname{sum}\left(\gamma_{1}^{2}\right)$

$0.429171 .6198 \operatorname{sum}(\gamma)$

0.451471 .4012 SST

0.474171 .1044
Fitted

$\gamma_{i s} \quad\left(\gamma_{i-1} \gamma_{i s}\right)^{2} \quad \gamma_{i s}^{2}$ $\mathrm{mJ} / \mathrm{m}^{2} \quad\left(\mathrm{~mJ} / \mathrm{m}^{2}\right)^{2}\left(\mathrm{~mJ} / \mathrm{m}^{2}\right)^{2}$

$72.1399 \quad 1.1 \mathrm{E}-06 \quad 5204.0132$

$\begin{array}{lll}72.1396 & 1.29 E-06 & 5203.9624\end{array}$

7.242808

$0.75221 \mathrm{mg} / \mathrm{m}^{2}$

$42.19444 \mathrm{~mJ} / \mathrm{m}^{2}$

$\begin{array}{llll}72.1389 & 1.78 E-06 & 5203.8218\end{array}$

$\begin{array}{lll}72.1372 & 2.76 \mathrm{E}-06 \quad 5203.5409\end{array}$

$\begin{array}{llll}72.1351 & 4.08 E-06 & 5203.1775\end{array}$

72.1307 6.88E-06 5202.4603

$72.1236 \quad 1.17 \mathrm{E}-05 \quad 5201.3215$

72.1071 2.37E-05 5198.739

$72.0754 \quad 4.75 E-05 \quad 5193.8649$

$72.0533 \quad 6.38 \mathrm{E}-05 \quad 5190.521 \mathrm{I}$

$72.0259 \quad 8.31 \mathrm{E}-05 \quad 5186.4165$

$\begin{array}{llll}71.9313 & 0.000141 & 5172.4012\end{array}$

$\begin{array}{lll}71.886 & 0.000163 & 5165.764\end{array}$

$\begin{array}{lll}71.635 & 0.00023 & 5129.3993\end{array}$

$71.41650 .000234 \quad 5098.1355$

$\begin{array}{llll}71.1184 & 0.000194 & 5055.8425\end{array}$ 


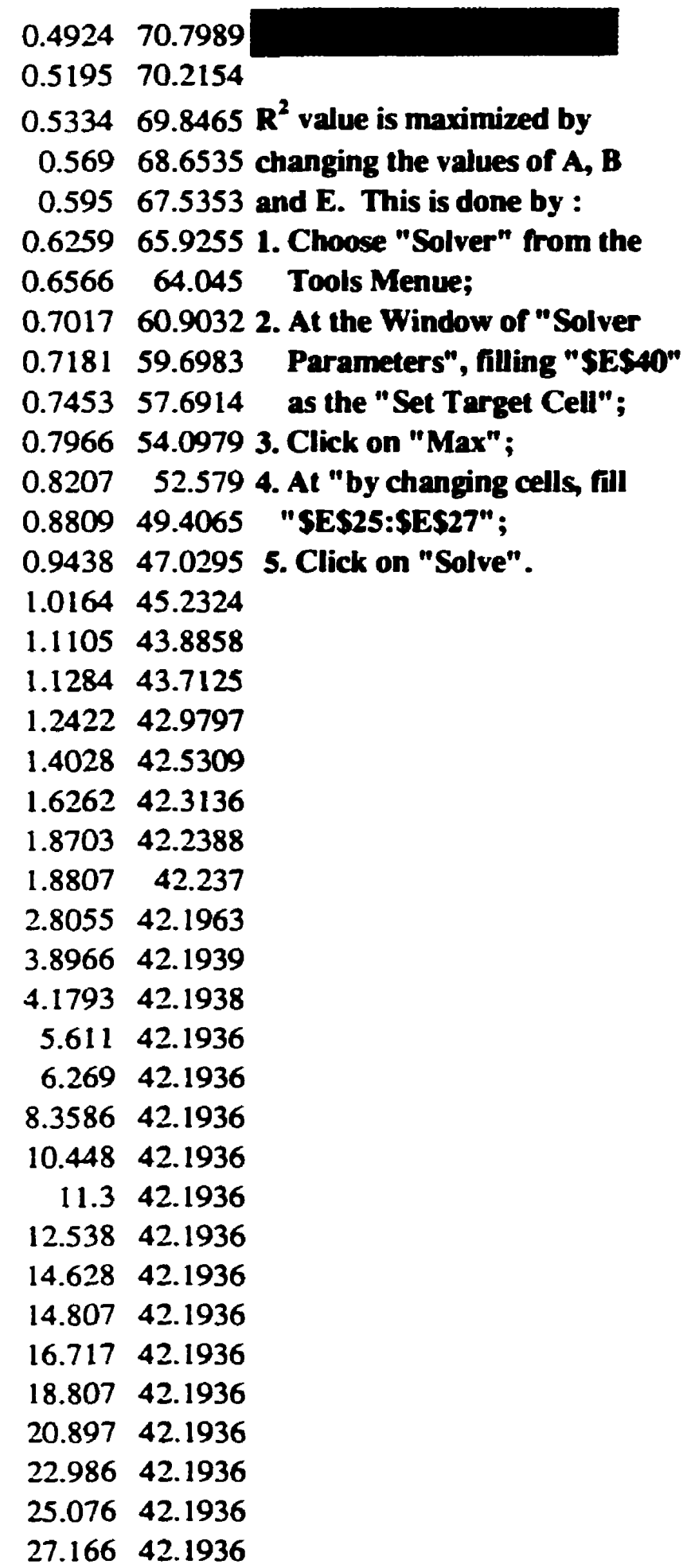

\begin{tabular}{rrr}
70.8102 & 0.000129 & 5012.4805 \\
70.2202 & $2.31 \mathrm{E}-05$ & 4930.2046 \\
69.8466 & $1.41 \mathrm{E}-08$ & 4878.5319 \\
\hline 68.6381 & 0.000237 & 4713.3055 \\
67.507 & 0.0008 & 4561.0212 \\
65.8836 & 0.00176 & 4346.1756 \\
63.9954 & 0.002464 & 4101.7617 \\
60.8586 & 0.001993 & 3709.2014 \\
59.6601 & 0.001457 & 3563.8817 \\
\hline 57.6674 & 0.000573 & 3328.2951 \\
54.1022 & $1.85 \mathrm{E}-05$ & 2926.5808 \\
\hline 52.593 & 0.000196 & 2764.5502 \\
49.4297 & 0.000538 & 2441.006 \\
47.0458 & 0.000266 & 2211.7703 \\
45.2354 & $8.61 \mathrm{E}-06$ & 2045.9733 \\
43.8767 & $8.39 \mathrm{E}-05$ & 1925.9669 \\
43.702 & 0.000111 & 1910.782 \\
\hline 42.9656 & 0.000199 & 1847.2534 \\
\hline 42.519 & 0.000142 & 1808.8796 \\
\hline 42.3065 & $4.99 \mathrm{E}-05$ & 1790.4396 \\
\hline 42.2352 & $1.23 \mathrm{E}-05$ & 1784.1121 \\
\hline 42.2336 & $1.16 \mathrm{E}-05$ & 1783.9673 \\
42.1966 & $8.97 \mathrm{E}-08$ & 1780.5281 \\
\hline 42.1946 & $5.71 \mathrm{E}-07$ & 1780.3236 \\
42.1946 & $6.14 \mathrm{E}-07$ & 1780.3146 \\
42.1945 & $6.85 \mathrm{E}-07$ & 1780.3018 \\
42.1944 & $6.93 \mathrm{E}-07$ & 1780.3008 \\
42.1944 & $6.99 \mathrm{E}-07$ & 1780.3 \\
42.1944 & $7 \mathrm{E}-07$ & 1780.2999 \\
42.1944 & $7 \mathrm{E}-07$ & 1780.2999 \\
42.1944 & $7 \mathrm{E}-07$ & 1780.2999 \\
42.1944 & $7 \mathrm{E}-07$ & 1780.2999 \\
42.1944 & $7 \mathrm{E}-07$ & 1780.2999 \\
42.1944 & $7 \mathrm{E}-07$ & 1780.2999 \\
42.1944 & $7 \mathrm{E}-07$ & 1780.2999 \\
42.1944 & $7 \mathrm{E}-07$ & 1780.2999 \\
42.1944 & $7 \mathrm{E}-07$ & 1780.2999 \\
42.1944 & $7 \mathrm{E}-07$ & 1780.2999 \\
42.1944 & $7 \mathrm{E}-07$ & 1780.2999
\end{tabular}




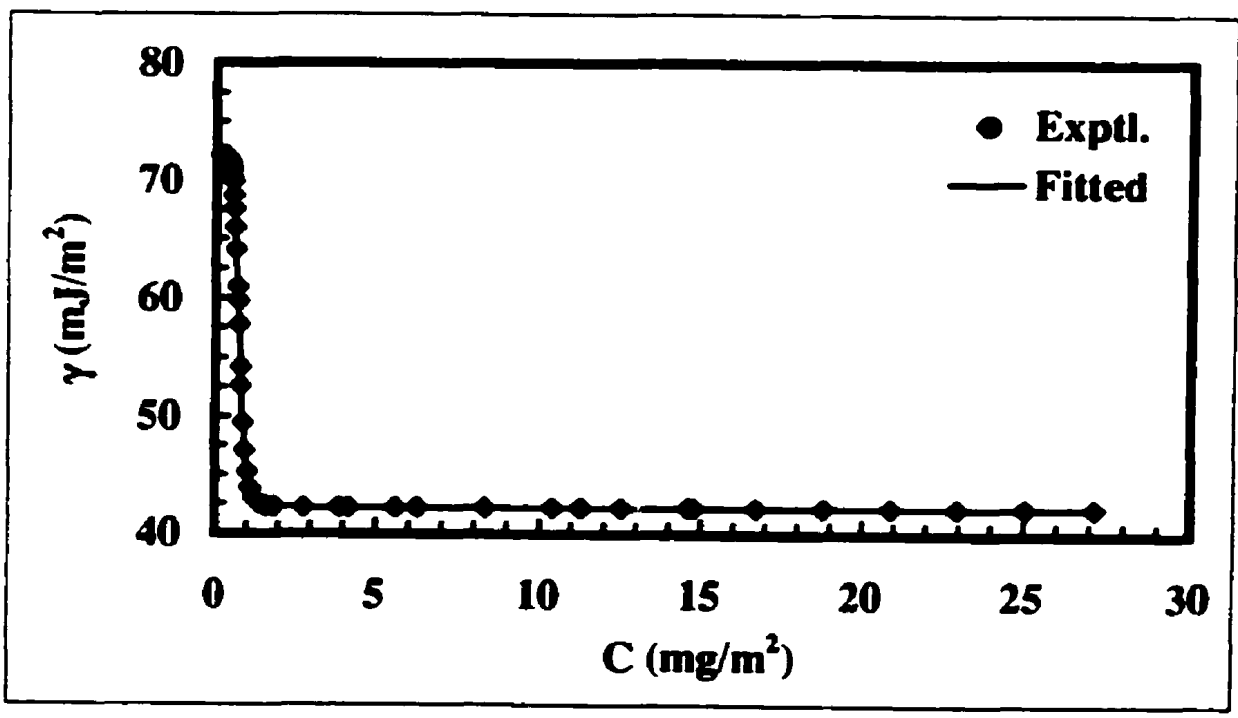

The final equation for the surface tension of PNIPAM at $25^{\circ} \mathrm{C}$ is:

$$
\gamma_{2 S C}(C)=42.1994+\frac{29.9456}{1+\left(\frac{C}{0.7522}\right)^{7.2428}}
$$

$\left(R^{2}=1.0000\right)$
Equation A2.5.8 
From Equation A2.53,

$$
\Gamma_{2 S C}(C)=0.3325 \operatorname{Ln} C+0.695
$$

Equation A2.5.3

it is obtained that

$$
C=e^{\frac{\Gamma-0.695}{0.3325}}=0.1237 e^{\frac{\Gamma}{0.3325}}
$$

Equation A2.5.9

Substituting Equation A2.5.9 into Equation A2.58,

$$
\gamma_{2 S C}(C)=42.1994+\frac{29.9456}{1+\left(\frac{C}{0.7522}\right)^{7.2428}}
$$

Equation $\mathbf{A 2 . 5 . 8}$

it gives:

$$
\gamma_{2 S C}(\Gamma)=42.1994+\frac{29.9456}{1+\left(\frac{0.1237 e^{\frac{r}{0.3325}}}{0.7522}\right)^{7.2428}}
$$

Simplifying the above equation, the surface tensions of aqueous PNIPAM solutions as a function of surface concentration is obtained as:

$$
\gamma_{2 S C}(\Gamma)=42.1944+\frac{29.9456}{1+2.0985 \times 10^{-6} e^{21.7829 \Gamma}}
$$

Or the surface concentration of PNIPAM at the air/water interface at $25^{\circ} \mathrm{C}$ as a function of surface tension is:

$$
\Gamma_{2 S C}(\gamma)=0.6002+0.0459 \operatorname{Ln} \frac{72.14-\gamma}{\gamma-42.1944}
$$

Equation A2.5.11

According to Equation A25.10,

\begin{tabular}{ll}
$\Gamma$ & \multicolumn{1}{r}{$\boldsymbol{r}$} \\
$\mathrm{mg} / \mathrm{m}^{2}$ & \multicolumn{1}{c}{$\mathrm{mJ} / \mathrm{m}^{2}$} \\
0.4 & 71.76259 \\
0.8 & 42.57521
\end{tabular}

According to Equation A2.5.11,

$$
\begin{array}{rl}
r & r \\
\mathrm{~mJ} / \mathrm{m}^{2} & \mathrm{~m} / \mathrm{m}^{2} \\
40.79 & \text { wNUM! } \\
43.02 & 0.763745 \\
43.38 & 0.746563
\end{array}
$$




\section{Appendix 2.6 Calculation of the Bulk Depletion}

The purpose of this calculation is to see the extent of the bulk depletion with L-6B at $25{ }^{\circ} \mathrm{C}$ as examples.

Please refer to the main text for the definitions of the symbols. For those not defined in the main text, the definitions are as follows:

$C_{\text {b0 }}$ represents the initial bulk concentration;

$\mathbf{t} \mathbf{m}$ represents the time required to reach meso-equilibrium surface tension;

$A_{m} \quad$ represents the drop surface area at $t=t_{m}$;

$V_{m}$ respresents the drop volume at $t=t \quad m$;

$C_{\text {bm }} \quad$ represents the equilibrium bulk concentration;

$\Gamma_{\mathrm{m}}$ represents the meso-equilibrium interfacial concentration of polymer,

t star represents $t^{*}$ in the Hua-Rosen equation;

$\Delta_{\mathrm{d}} \quad$ represents the percentage depletion in the bulk solution.

According to the relationship developed from Kawaguchi's data, at $25^{\circ} \mathrm{C}$ :

$$
\Gamma=\left(0.6002+0.0459 \cdot \ln \left(\frac{\gamma_{0}-\gamma}{\gamma-42.19}\right)\right) \cdot \frac{m g}{m^{2}} \quad \text { Equation A2.6.1 }
$$

And the Hua-Rosen Equation [1, 2]:

$$
\gamma(t)=\gamma_{m}+\frac{\gamma_{0}-\gamma_{m}}{1+\left(\frac{t}{t_{\text {star }}}\right)^{n}}
$$

The time required to reach meso-equilibrium surface tension $[1,2]$ :

$$
t^{=t} \operatorname{star} \cdot 10^{\frac{1}{n}}
$$


Then, substitue $\gamma$ in Equation A2.6.1 with $\gamma(t)$ prdicted by Equation A2.6.2, the surface concentration of polymer at any time $t$ is:

$$
\Gamma(t)=\left[0.6002+0.0459 \cdot \ln \left[\frac{\gamma_{0}-\left[\gamma_{m}+\frac{\gamma_{0}-\gamma_{m}}{1+\left(\frac{t}{t_{s t a r}}\right)^{n}}\right]}{\gamma_{m}+\frac{\gamma_{0}-\gamma_{m}}{1+\left(\frac{t}{t_{\text {star }}}\right)^{\mathrm{n}}}-42.19}\right] \cdot \frac{\mathrm{mg}}{\mathrm{m}^{2}}\right.
$$

Rearrange the above equation, we have:

$$
\Gamma(t)=\left[0.6002+0.0459 \cdot \ln \left[\frac{\left(\gamma_{0}-\gamma_{m}\right) \cdot\left(\frac{t}{t_{\text {star }}}\right)^{\mathrm{n}}}{\gamma_{0}-42.19+\left(\gamma_{m}-42.19\right) \cdot\left(\frac{\mathrm{t}}{\mathrm{t}_{\text {star }}}\right)^{\mathrm{n}}}\right]\right] \cdot \frac{\mathrm{mg}}{\mathrm{m}^{2}}
$$

Equation A2.6.3

The surface concentration at the meso-equilibrium state when the first time $t=t_{m}$

$$
\Gamma_{\mathrm{m}}=\left[0.6002+0.0459 \cdot \ln \left[\frac{\left(\gamma_{\mathrm{s}}-\gamma_{\mathrm{m}}\right) \cdot\left(\frac{\mathrm{t}_{\mathrm{m}}}{\mathrm{t}_{\mathrm{star}}}\right)^{\mathrm{n}}}{\gamma_{\mathrm{s}}-42.19+\left(\gamma_{\mathrm{m}}-42.19\right) \cdot\left(\frac{\mathrm{t}_{\mathrm{m}}}{\mathrm{t}_{\mathrm{star}}}\right)^{\mathrm{n}}}\right]\right] \cdot \frac{\mathrm{mg}}{\mathrm{m}^{2}}
$$


The bulk concentration at the meso-equilibrium state is:

$$
C_{b m}=C_{b 0}-\frac{\Gamma_{m} \cdot A_{m}}{V_{m}}
$$

Percentage depletion in the bulk solution is:

$$
\Delta_{d}=\frac{C_{b 0}-C_{b m}}{C_{b 0}} \cdot 100 \% \quad \text { Equation A2.6.6 }
$$

According to Jasper [3]: $\quad \gamma_{0}:=72.14 \mathrm{~mJ} / \mathrm{m}^{2}$

According to the experiments and Table 2.2 in the main text, we have for L-6B at $25^{\circ} \mathrm{C}$ :

When $C_{\text {bo }}=10 \mathrm{ppm}$ :

$$
C_{b 0}:=10000 \cdot \frac{\mathrm{mg}}{\mathrm{m}^{3}} \quad \gamma_{\mathrm{m}}:=43.25 \quad \frac{\mathrm{mJ}}{\mathrm{m}^{2}} \quad \mathrm{n}:=4.2017 \quad \mathrm{t}_{\mathrm{star}}:=422.1 \cdot \mathrm{s}
$$

Then, $t_{\mathrm{m}}:=\mathrm{t}_{\mathrm{star}} \cdot 10^{\frac{1}{\mathrm{n}}} \quad \mathrm{t}_{\mathrm{m}}=730.154 \cdot \mathrm{s}$

$$
\begin{gathered}
A_{m}:=0.2876 \cdot 10^{-4} \cdot \mathrm{m}^{2} \quad V_{m}:=0.0175 \cdot 10^{-6} \cdot \mathrm{m}^{3} \\
\Gamma_{m}:=\left[\begin{array}{c}
\left(\gamma_{0}-\gamma_{\mathrm{m}}\right) \cdot\left(\frac{\mathrm{t}_{\mathrm{m}}}{\mathrm{t}_{\mathrm{star}}}\right)^{\mathrm{n}} \\
0.6002+0.0459 \cdot \ln \left[\frac{\gamma_{0}-42.19+\left(\gamma_{\mathrm{m}}-42.19\right) \cdot\left(\frac{\mathrm{t}_{\mathrm{m}}}{\mathrm{t}_{\mathrm{star}}}\right)^{\mathrm{n}}}{\gamma^{2}}\right]
\end{array}\right] \frac{\mathrm{mg}}{\mathrm{m}^{2}}
\end{gathered}
$$




$$
\begin{array}{ll}
\Gamma_{\mathrm{m}}=6.903 \cdot 10^{-7} \cdot \mathrm{kg} \cdot \mathrm{m} & -2 \\
C_{\mathrm{bm}}:=C_{b 0}-\frac{\Gamma_{\mathrm{m}} \cdot \mathrm{A}_{\mathrm{m}}}{V_{\mathrm{m}}} & C_{\mathrm{bm}}=8.865 \cdot \mathrm{ppm} \\
\Delta_{\mathrm{d}}:=\frac{C_{b 0}-C_{b m}}{C_{b 0}} \cdot 100 \% & \Delta_{\mathrm{d}}=11.345 \cdot \%
\end{array}
$$

When $C_{b 0}=30 \mathrm{ppm}$ :

$$
\mathrm{C}_{\mathrm{b0}}:=30000 \cdot \frac{\mathrm{mg}}{\mathrm{m}^{3}} \quad \gamma_{\mathrm{m}}:=43.10 \quad \frac{\mathrm{mJ}}{\mathrm{m}^{2}} \quad \mathrm{n}:=2.4113 \quad \mathrm{t}_{\text {star }}:=56.8 \cdot \mathrm{s}
$$

Then, $\quad t_{\mathrm{m}}:=\mathrm{t}_{\mathrm{star}} \cdot 10^{\frac{1}{\mathrm{n}}} \quad \mathrm{t}_{\mathrm{m}}=147.592 \cdot \mathrm{s}$

$$
\begin{aligned}
& A_{m}:=0.3928 \cdot 10^{-4} \cdot \mathrm{m}^{2} \quad v_{m}:=0.0250 \cdot 10^{-6} \cdot \mathrm{m}^{3} \\
& \Gamma_{m}:=\left[0.6002+0.0459 \cdot \ln \left[\frac{\left(\gamma_{0}-\gamma_{m}\right) \cdot\left(\frac{t_{m}}{t_{\text {star }}}\right)^{n}}{\gamma_{0}-42.19+\left(\gamma_{m}-42.19\right) \cdot\left(\frac{t_{m}}{t_{\text {star }}}\right)^{n}}\right] \cdot \frac{m g}{m^{2}}\right. \\
& \Gamma_{\mathrm{m}}=6.923 \cdot 10^{-7} \cdot \mathrm{kg} \cdot \mathrm{m}^{-2} \\
& C_{b m}:=C_{b 0}-\frac{\Gamma_{m^{*}} A_{m}}{V_{m}^{m}} \quad C_{b m}=28.912 \cdot p p m \\
& \Delta_{d}:=\frac{C_{b 0}-C_{b m}}{C_{b 0}} \cdot 100 \% \quad \Delta_{d}=3.626 . \%
\end{aligned}
$$


When $C_{b 0}=40 \mathrm{ppm}$ :

$$
C_{b 0}:=40000 \cdot \frac{\mathrm{mg}}{\mathrm{m}^{3}} \quad \gamma_{\mathrm{m}}:=43.02 \quad \frac{\mathrm{mJ}}{\mathrm{m}^{2}} \quad \mathrm{n}:=3.6673 \quad \mathrm{t}_{\mathrm{star}}:=35.5 \cdot \mathrm{s}
$$

Then, $\quad t_{m}:=t_{\operatorname{star}} \cdot 10^{\frac{1}{n}} \quad t_{m}=66.513 \cdot s$

$$
A_{m}:=0.3754 \cdot 10^{-4} \cdot \mathrm{m}^{2} \quad v_{m}:=0.0244 \cdot 10^{-6} \cdot \mathrm{m}^{3}
$$

$$
\begin{aligned}
& \Gamma_{\mathrm{m}}:=\left[0.6002+0.0459 \cdot \ln \left[\frac{\left(\gamma_{0}-\gamma_{\mathrm{m}}\right) \cdot\left(\frac{t_{\mathrm{m}}}{\mathrm{t}_{\mathrm{star}}}\right)^{\mathrm{n}}}{\gamma_{\mathrm{o}}-42.19+\left(\gamma_{\mathrm{m}}-42.19\right) \cdot\left(\frac{\mathrm{t}_{\mathrm{m}}}{\mathrm{t}_{\mathrm{star}}}\right)^{\mathrm{n}}}\right]\right] \cdot \frac{\mathrm{mg}}{\mathrm{m}^{2}} \\
& \Gamma_{\mathrm{m}}=6.934 \cdot 10^{-7} \cdot \mathrm{kg} \cdot \mathrm{m}^{-2} \\
& C_{\mathrm{bm}}:=\mathrm{C}_{\mathrm{b0}}-\frac{\Gamma_{\mathrm{m}} \cdot \mathrm{A}_{\mathrm{m}}}{\mathrm{V}_{\mathrm{m}}} \quad \mathrm{C}_{\mathrm{bm}}=38.933 \cdot \mathrm{ppm} \\
& \Delta_{\mathrm{d}}:=\frac{\mathrm{C}_{\mathrm{b} 0}-\mathrm{C}_{\mathrm{bm}} \cdot 100 \%}{\mathrm{C}_{\mathrm{b} 0}} \quad \Delta_{\mathrm{d}}=2.667 \cdot \%
\end{aligned}
$$

When $C_{60}=50 \mathrm{ppm}$ :

$$
C_{b 0}:=50000 \cdot \frac{\mathrm{mg}}{\mathrm{m}^{3}} \quad \gamma_{\mathrm{m}}:=42.44 \frac{\mathrm{mJ}}{\mathrm{m}^{2}} \quad \mathrm{n}:=3.0042 \quad t_{\text {star }}:=6 . \mathrm{s}
$$




$$
\begin{aligned}
& \text { Then, } \quad t_{\mathrm{m}}:=t_{\mathrm{star}} \cdot 10^{\frac{1}{n}} \quad t_{\mathrm{m}}=12.913 \cdot \mathrm{s} \\
& A_{m}:=0.3631 \cdot 10^{-4} \cdot m^{2} \quad V_{m}:=0.0233 \cdot 10^{-6} \cdot m^{3} \\
& \Gamma_{m}:=\left[0.6002+0.0459 \cdot \ln \left[\frac{\left(\gamma_{0}-\gamma_{m}\right) \cdot\left(\frac{t_{m}}{t_{s t a r}}\right)^{n}}{\gamma_{0}-42.19+\left(\gamma_{m}-42.19\right) \cdot\left(\frac{t_{\mathrm{m}}}{t_{\mathrm{star}}}\right)^{n}}\right]\right] \cdot \frac{\mathrm{mg}}{\mathrm{m}^{2}} \\
& \Gamma_{\mathrm{m}}=7.018 \cdot 10^{-7} \cdot \mathrm{kg} \cdot \mathrm{m}^{-2} \\
& C_{b m}:=C_{b 0}-\frac{\Gamma_{m} \cdot A_{m}}{V_{m}} \quad C_{b m}=48.906 \cdot p p m \\
& \Delta_{d}:=\frac{C_{b 0}-C_{b m}}{C_{b 0}} \cdot 100 \% \quad \Delta_{d}=2.187 \cdot \%
\end{aligned}
$$

\section{References:}

1. Hua, X. Y. and Rosen, M. J., J. Colloid Interface Sci., 124, 652 (1988).

2. Hua, X. Y. and Rosen, M. J., J. Colloid Interface Sci., 141, 180 (1991).

3. Jasper, J. J., J. Physical and Chemical Reference Data, 1, 948 (1972). 


\section{Appendix 2.7 Calculation of the Number of PNIPAM Chains per Phase-separated Particle}

The purpose of this calculation is to find the number of PNIPAM chains per phase-separated particle.

$P_{p} \quad$ density of a phase-separated particle;

$W_{p} \quad$ mass of a phase-separated particle;

$V_{p} \quad$ volume of a phase-separated particle;

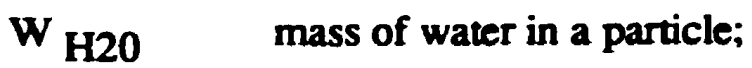

W PNIPAM mass of PNIPAM in a particle;

$f_{\text {wH20 mass fraction of water in a particle; }}$

f wPNIPAM mass fraction of PNIPAM in a particle;

$\rho$ PNIPAM density of PNIPAM polymer [1], $\quad \rho_{\text {PNIPAM }}=1.07 \cdot \frac{\mathrm{gm}}{\mathrm{cm}^{3}}$

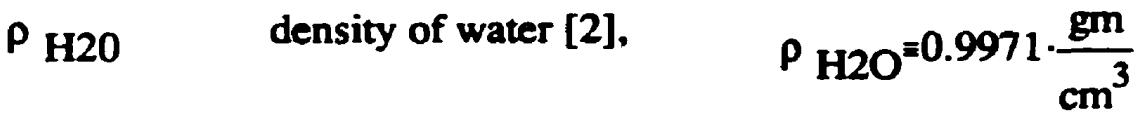

$M_{w}$ the weight average molecular weight of PNIPAM (L-6B or

d $\quad$-4);

diameter of a phase-separated particle of L-6B or L-4 measured by dynamic scattering in $\mathrm{nm}$.

$$
\begin{aligned}
& \rho_{p}=\frac{W_{p}}{V_{p}} \\
& v_{p}=\frac{w_{H 2 O}}{\rho_{H 2 O}}+\frac{w_{\text {PNIPAM }}}{\rho_{\text {PNIPAM }}} \\
& \text { Equation A2.7.2 } \\
& W_{p}=W_{H 2 O}+W_{\text {PNIPAM }}=f_{w H 2 O} \cdot W_{p}+f_{\text {wPNIPAM }} \cdot W_{p}
\end{aligned}
$$


Therefore,

$$
\rho_{p}=\frac{w_{p}}{f_{w H 20} \frac{w_{p}}{\rho_{H 2 O}}+f_{\text {wPNIPAM }} \frac{w_{p}}{\rho_{\text {PNIPAM }}}}=\frac{1}{f_{w H 2 O}} \frac{f_{\text {wPNIPAM }}}{\rho_{\text {H2O }}}+\frac{\rho_{\text {PNIPAM }}}{\rho_{\text {PNIPA }}}
$$

According to Dong and Hoffman [3], the water content in a phase-separated PNIPAM particle is approximately $30 \%$. That is,

$$
f_{w H 2 O}:=0.3
$$

Then,

$$
\text { f wPNIPAM }:=0.7
$$

Therefore, the density of the particle is:

$$
\rho_{\mathrm{p}}:=\frac{1}{\frac{f_{w H 2 O}}{\rho_{\mathrm{H} 2 \mathrm{O}}}+\frac{f_{w P N I P A M}}{\rho_{\text {PNIPAM }}}} \quad \rho_{\mathrm{p}}=1.047 \cdot 10^{3} \cdot \mathrm{kg} \cdot \mathrm{m}^{-3}
$$

Assuming a spherical shape of a phase-separated PNIPAM particle, then

$$
V_{p}=\frac{\pi d^{3}}{6}
$$

Equation A2.7.4

Then, the mass of phase-separated PNIPAM per particle is:

$W_{\text {PNIPAM }}=f_{\text {wPNIPAM }}\left(V_{p} \cdot \rho_{p}\right)=\frac{\pi d^{3}}{6} \cdot f_{\text {wPNIPAM }} \cdot \rho_{p}$ Equation A2.7.5

The number of PNIPAM chains per phase-separated particle is:

$$
\mathrm{N}=\frac{\mathrm{W}_{\text {PNIPAM }}}{\mathbf{M}_{\mathbf{w}}} \cdot \mathrm{N}_{\mathrm{av}}
$$


The diameters of phase-seaprated L-6B particles at $40^{\circ} \mathrm{C}$ shaown in Matrix $\mathrm{d}$ are $d:=\left[\begin{array}{c}64.6 \\ 73.3 \\ 81.4 \\ 83.5 \\ 88.2 \\ 98.84 \\ 105.6 \\ 116.7 \\ 134.9\end{array}\right] \cdot 10^{-7} \cdot \mathrm{cm} \quad i:=0 . .8$

$$
M_{\text {wL6B }}:=5.47 \cdot 10^{5} \cdot \frac{\mathrm{gm}}{\mathrm{mole}}
$$

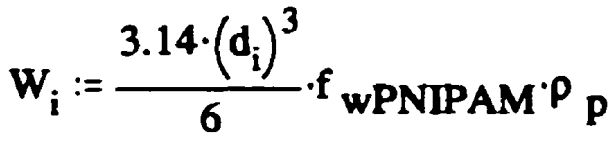

$\mathbf{N}_{\mathrm{i}}:=\frac{\mathrm{w}_{\mathrm{i}}}{\mathbf{M}_{\text {wL6B }}} \cdot \mathbf{N}_{\text {av }}$

The number of PNIPAM chains in the phase separated L-6B particles corresponding to the diameters listed above in the array $\mathrm{d}$ are

$$
N=\left[\begin{array}{l}
113.841 \\
166.308 \\
227.758 \\
245.844 \\
289.739 \\
407.755 \\
497.27 \\
671.14 \\
1.037 \cdot 10^{3}
\end{array}\right]
$$


The diameters of phase-seaprated L-4 particles at $40^{\circ} \mathrm{C}$ shaown in Matrix $\mathrm{d}$ are:

$$
\mathrm{d}:=\left[\begin{array}{c}
79.7 \\
89.3 \\
101 \\
108.7 \\
114.8 \\
131.9 \\
133.2 \\
141.2 \\
153.7
\end{array}\right] \cdot 10^{-7} \cdot \mathrm{cm} \quad \mathrm{i}:=0.8 \quad \mathbf{M}_{\mathbf{w L}}:=1.31 \cdot 10^{4} \cdot \frac{\mathrm{gm}}{\mathrm{mole}}
$$

The number of PNIPAM chains in the phase separated L-4

particles corresponding to the the diameters listed above in the array $d$ are:

$$
\mathrm{N}=\left[\begin{array}{c}
8.927 \cdot 10^{3} \\
1.256 \cdot 10^{4} \\
1.817 \cdot 10^{4} \\
2.265 \cdot 10^{4} \\
2.668 \cdot 10^{4} \\
4.046 \cdot 10^{4} \\
4.167 \cdot 10^{4} \\
4.964 \cdot 10^{4} \\
6.402 \cdot 10^{4}
\end{array}\right]
$$

References:

1. CRC Handbook of Chemistry and Physics, 69th Edition.

2. Sewell, J. H., J. of Applied Polymer Science, 17, 1741 (1973).

3. L. C. Dong and A. S. Hoffman, J. Controlled Release, 13, 21 (1990). 


\title{
Chapter 3
}

\section{The Application of Polymer Adsorption Models to Dynamic Surface Tension}

\begin{abstract}
The Langmuir kinetics model and van Eijk and Cohen Stuart's spreading disk model were used to predict the dynamic surface tension of aqueous poly $(N$ isopropylacrylamide). Both published experimental results and calculations based on self-consistent field theory were used to convert the concentrations of adsorbed polymer to surface tension. The modeling could predict the slopes of surface tension versus log time curves, however the times scales for surface tension lowering could not be modeled for a series of polymer concentrations using a single set of model parameters. Oscillating drop experiments in which the surface area of pendent drops was varied sinusoidally by pumping solution in and out of the drop were modeled. The experimental steady-state surface tension oscillations were less than the predicted values indicating that the adsorbed layer could reconfigure to compensate for an expanding interface.
\end{abstract}

Keyword: kinetic model, adsorption, poly( $N$-isopropylacrylamide), dynamic surface tension. 


\subsection{Introduction}

In a previous publication, we reported extensive dynamic surface tension data for aqueous poly( $N$-isopropylacrylamide) [1], which included results from the analysis of both static pendent drops (SPD) and from oscillating pendent drops (OPD). The static drop results showed classic S-shaped curves of surface tension versus drop age. By contrast, in the OPD experiments, pumping solution in and out of pendent drops generated sinusoidal changes in drop area. The changing surface area generated initial large swings in surface tension, which decayed with time to steady-state sinusoidal surface tension oscillations (see lower half of Figure 3. 7 for an example). In this paper, we report attermpts to fit our results to published adsorption models with a view to gaining insight into the mechanisms controlling the dynamic surface tension behavior. Our modeling involved two steps. In the first step, polymer adsorption kinetic models were employed to generate time dependent mass concentrations of polymer at the air/ water interface. In the second step, results either from self-consistent field calculations or from published data were used to convert adsorbed polymer concentrations to the corresponding surface tensions. The following paragraphs introduce the models applied in this work.

The literature is replete with theoretical and experimental investigations of polymers at interfaces [2]. It is generally accepted that adsorption of polymers (proteins, synthetic polymers) is often irreversible $[2,3,4,5,6]$. In such cases, 
equilibrium isotherm models such as the Gibbs and Langmuir isotherms are difficult to apply $[7,8,9,10,11,12,13,14,15]$. Nevertheless, thermodynamics, together with Monte Carlo simulations, have been a major approach for modeling adsorbed layer configurations [2].

The kinetics of polymer adsorption and desorption has been less explored than the equilibrium composition and layer structure because the dynamic characterization of adsorption is difficult. Recently, infrared spectroscopy [16, 17] and reflectrometry [18] have been used to explore adsorption dynamics. For example, Frantz and Granik reported the adsorption-desorption kinetics of polystyrene from cyclohexane onto silicon oxide through in situ Fouriertransform infrared spectroscopy [16]. They showed that diffusion to and adsorption onto an initially bare surface is rapid and surface rearrangements are slow and history dependent.

Polymer adsorption kinetics are usually modeled either by simple mass transport models or by computational complex simulations such as random sequential adsorption models $[3,4,5,6,15,19,20]$. In the latter models, polymers or colloidal particles are treated as disks, which randomly deposit onto an interface. Recent versions of these models allow the disks to spread instantaneously and symmetrically giving a larger size after adsorption $[6,15]$. Although successful at simulating experimental data, particularly at low coverage, random sequential models usually are computationally intensive $[3,20]$. 
Mass transport controlled polymer adsorption has been described for welldefined flows such as the rotating disk or stagnation point flow $[21,22]$. Similarly, equations describing the initial rates of adsorption in the absence of flow have been given by Ward and Tordi [23]. In many practical cases flow conditions are poorly defined, rather primitive models are applied. One such model is the "Langmuir kinetics model", which derives its name because the form of the equation is the same as the Langmuir adsorption isotherm [24]. The model is

$$
\frac{d \theta}{d t}=k_{1}(1-\theta)\left(n_{0}-\theta\right)-k_{2} \theta \quad \text { Equation } 3.1
$$

where $\theta$ is the fraction of surface covered, $n_{0}$ is the mass of polymer per unit volume in solution divided by the maximum adsorption per unit volume; $k_{l}$ is the adsorption rate constant and $k_{2}$ is the desorption rate constant. The Langmuir kinetics model works well for polymers that behave as solid spheres, that is, polymers which do not rearrange on the surface after adsorption and polymers whose size in solution is independent of polymer concentration.

Recently, van Eijk and Cohen Stuart proposed a simple kinetic model, herein called the EC model, which accounts for the reconfiguration of adsorbed polymer [25]. The model is cast in terms of the flux of polymer to the surface and analytical solutions are given for the case of constant flux, which can be obtained in stagnation point flow. Specifically, the EC model describes the deposition of 
disks on a surface. Once deposited, the disks with an initial contact area, $a_{o}$, expand until they reach a maximum contact area of $\left(a_{0}+\alpha\right)$, or until there is no free surface. The expanding disk idea follows concepts discussed by Pefferkorn and Elaissari [26].

Finally, there have been very few models for predicting surface tension kinetics. The Hua-Rosen equation is a three-parameter model which, although empirical, gives very good fits to our experimental data [1]. However, the fitting parameters give little insight into mechanisms.

In this work we apply the Langmuir kinetics model and the EC model to our surface tension data. Further, since the flux is not known in our work the EC model was modified slightly - this is described in the next section together with the conversion of adsorbed amounts to surface tension. Finally, we present a simple treatment of OPD experiments. 


\subsection{Theoretical Aspects}

\subsubsection{The Eijk \& Cohen Stuart (EC) Model}

The EC model [25] accounts for the reconformation of adsorbed polymer by considering the adsorption and subsequent spreading of disks. The model uses the following expression to describe the time dependence of the contact area of an adsorbed disk.

$$
a(t, u)=a_{0}+\alpha\left(1-e^{-\frac{(t-u)}{\tau_{0}}}\right)
$$

Equation 3.2

where $a_{0}$ is the initial area; $\alpha$ is the maximum area which a single disk can expand, $t$ is the elapsed time from the beginning of the experiment; $u$ is the elapsed time the disk has been adsorbed, and $\tau_{s}$ is the time constant $e$ following equation for mass

transport

$$
\frac{d \Gamma}{d t}=J \beta
$$

where $\Gamma$ is the interfacial concentration of adsorbed polymer and $\beta$ is the fraction of free interfacial area available to an incoming polymer chain. $\beta$ equals 1 initially and goes to 0 when the surface is saturated. van Eijk and Cohen Stuar give the time dependence of $\beta$ as: 


$$
\beta(t)=1-J \int_{0}^{1} \beta(u)\left[a_{0}+\alpha\left(1-e^{-(t-s) / \tau}\right)\right] d u \quad \text { Equation } 3.4
$$

Flux is not known in our experiments so it was assumed that $J$ was proportional to the bulk polymer concentration $C_{b}$ that is, $J=k C_{b}$ where $k$ is a mass transport constant. Equation 3.3 becomes

$$
\frac{d \Gamma}{d t}=k C_{b} \beta
$$

Equation 3.5

In the pendent drop experiments, the drop volume is finite and adsorption leads to depletion of dissolved polymer. This effect is significant when the polymer concentration is less than $20 \mathrm{ppm}$ (see Appendix 2.6). For more concentrated solutions $k$ and $C_{b}$ were assumed to be constant.

The concentration of adsorbed polymer, $\Gamma$, can be calculated as:

$$
\Gamma=k C_{b} \int_{0}^{t} \beta(u) d u
$$

Van Eijk and Cohen's analytical solutions to $B(u)$ can be used. For convenience, these are reproduced in Table 3.1. 
Table 3. 1 Solutions to $\beta(t)$ (see Equation 11 by van Eijk and Cohen [25]).

\begin{tabular}{|c|c|}
\hline$D^{*}$ & $\beta(t)$ \\
\hline 0 & $\left(1+\frac{1-k C_{b} \tau_{s} a_{0}}{2} \frac{t}{\tau_{s}}\right) e^{\frac{1+k C_{s} s_{s} a_{0} t}{2} \tau_{s}}$ \\
\hline$>0$ & $\frac{k C_{b} \tau_{s} a_{0}-1+\sqrt{D}}{2 \sqrt{D}} e^{\frac{1+k C_{s} \tau_{s}+\sqrt{D} r_{s}}{2} \tau_{s}}-\frac{k C_{b} \tau_{s} a_{0}-1-\sqrt{D}}{2 \sqrt{D}} e^{\frac{1+k C_{s} \tau_{s} a_{0}-\sqrt{D} s_{s}}{2} \tau_{s}}$ \\
\hline$<0$ & $\left(\cos \frac{t \sqrt{-D}}{2 \tau_{s}}+\frac{1-k C_{b} \tau_{s} a_{0}}{\sqrt{-D}} \sin \frac{t \sqrt{-D}}{2 \tau_{s}}\right) e^{\frac{1+k C_{s} s_{s} s_{s} s}{2 \tau_{s}}}$ \\
\hline
\end{tabular}

* $D=\left(1+k C_{b} \tau_{s} a_{0}\right)^{2}-4 k C_{\diamond} \tau_{s} a_{0}\left(1+\frac{\alpha}{a_{0}}\right)$

Irreversible polymer adsorption implies that $\Gamma$ must increase to a maximum, constant value. Evaluating the above expressions for the EC model yields $\Gamma$ versus time curves displaying a maximum (see Appendix 3.1 for more details). Following van Eijk and Cohen Stuart's approach, we assumed that the above expressions are valid until $\Gamma$ reaches a maximum value, $\Gamma_{\max }$, after which no changes in $\Gamma$ occur.

\subsubsection{Relating surface concentration to surface tension ( $\Gamma$-to-y)}

Polymer adsorption models give surface concentration as a function of time whereas our experimental data are surface tensions versus time. Two 
approaches are used herein to convert surface concentration to surface tension. The first method involves interpolation of experimental data whereas the second is theoretical.

The first approach is based on the work of Kawaguchi and co-workers [27] who reported both the surface pressure and the surface concentration, measured by ellipsometry, as functions of the amount of PNIPAM spread at the air/water interface in a Langmuir trough. We have taken their results and constructed plots of surface tension (equal to surface tension of pure water minus surface pressure) versus surface concentration at 16 and $31{ }^{\circ} \mathrm{C}$. Linear interpolation was employed to generate the corresponding curve at $25^{\circ} \mathrm{C}$. Details of the fitting are given elsewhere (see Appendix 2.5) and the result is given by the following equation:

$$
\gamma_{2 S C}(\Gamma)=42.19+\frac{29.95}{1+2.10 \times 10^{-6} e^{21.7 \times 295}}
$$

\section{Equation 3.7}

where $\Gamma$ is the concentration of polymer at the interface divided by $\mathrm{mg} / \mathrm{m}^{2}$. Equation 3.7 is plotted in Figure 3. 1. The surface tension is most sensitive to $\Gamma$ in the intermediate range 0.4 and $0.8 \mathrm{mg} / \mathrm{m}^{2}$ ). 


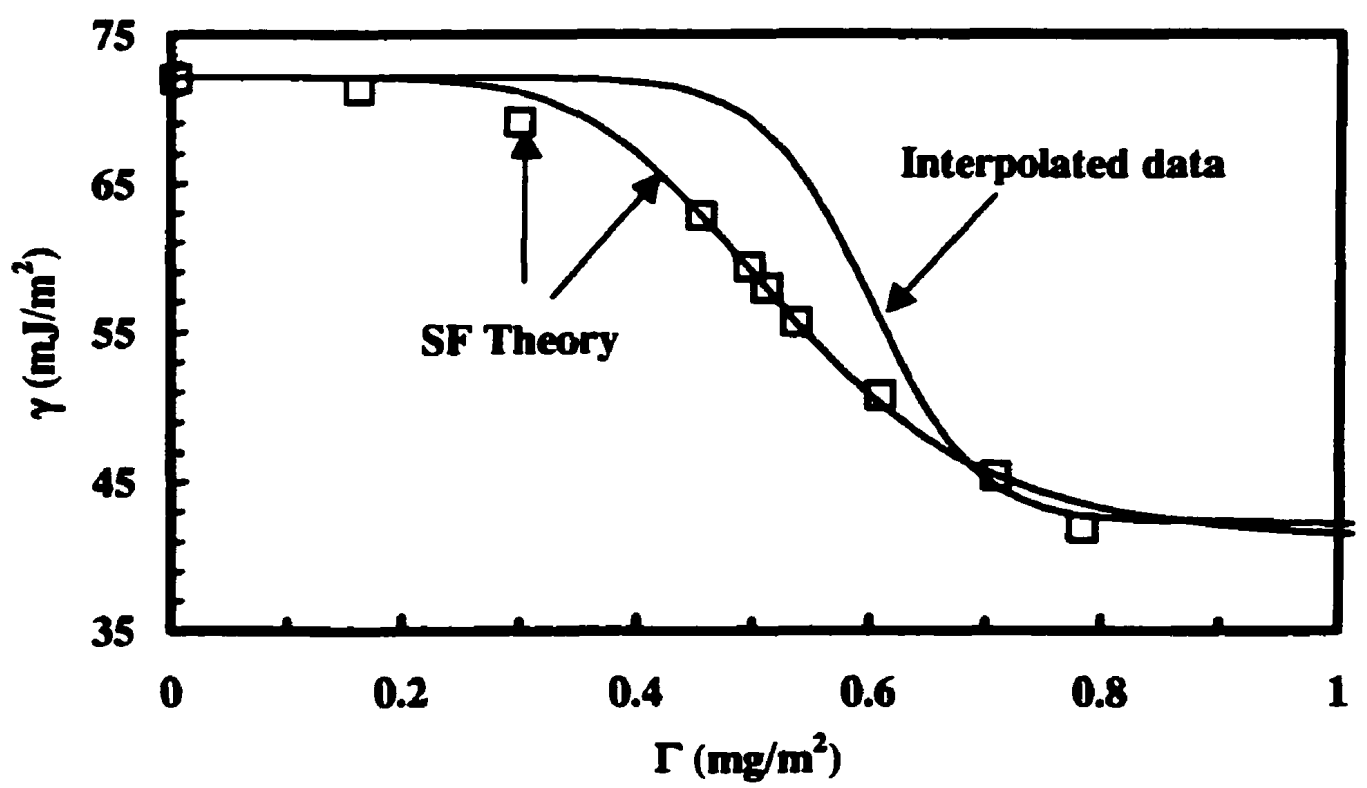

Figure 3.1 Surface tension of aqueous PNIPAM as a function of surface concentration interpolated from Kawaguchi's data [27] and from self-consistent field theory. The parameters in the SCF are: interaction parameters (kT/site) water/air 6.3. polymer/air 2.435, polymer/water 0.45 ; lattice depth 30; chain length 100; and area per site $0.3 \mathrm{~nm}^{2}$.

Also shown in Figure 3. 1 are the results of mean field calculations using Scheutjens-Fleer self-consistent field (SCF) theory [28] implemented in the program Goliad (version 2.1.0.0 kindly provided by Cosgrove and Fleer). The water/surface interaction parameter was chosen to give the surface tension of pure water. Similarly, the polymer/surface interaction parameter was chosen to give the minimum surface tension of $42 \mathrm{~mJ} / \mathrm{m}^{2}$ when the polymer volume fraction was high. The other program parameters are summarized in the figure caption. SCF theory predicted a more gradual decrease in surface tension with increasing surface concentration than did the curve interpolated from Kawaguchi's data. The 
following equation shows the empirical fit through the SCF results, which was required for modeling.

$$
\gamma(\Gamma)=40.8085+\frac{72.14-40.8085}{1+\left(\frac{\Gamma}{0.527}\right)^{5.5032}} \quad \text { Equation } 3.8
$$

\subsubsection{Modeling Oscillating Pendent Drop (OPD) Experiments}

We have reported dynamic pendent drop experiments in which drop volume oscillated linearly with time to induce a sinusoidal change in drop surface area. The objective of this section is to present a simple mass transport model for these experiments. It is assumed that adsorbed polymer does neither reconfigure $\left(\mathrm{a}(\mathrm{t}, \mathrm{u})=a_{0}\right)$ nor desorbs

Since surface area is not constant, the adsorbed polymer concentration must be expressed as the following function of the mass of adsorbed polymer, $w$, and the total surface area, $A$, which are both functions of time.

$$
\Gamma(t)=\frac{w(t)}{A(t)}
$$

The mass of adsorbed polymer can be expressed as the following function where $\beta$ is the fraction of surface which is not covered with adsorbed polymer and $a_{0}$ is the area occupied by adsorbed polymer expressed in $\mathrm{m}^{2} / \mathrm{mg}$. 


$$
w(t)=\frac{1-\beta(t)}{a_{0}} A(t)
$$

Combining the last two equations gives the time dependence of the concentration of adsorbed polymer.

$$
\Gamma(t)=\frac{1-\beta(t)}{a_{0}}
$$

An analytical expression for $\beta(t)$ is now derived. During an infinitesimal increase of time, $d u$, the mass of adsorbed polymer, $d w$, is assumed to be given by:

$$
d w=k C_{b}(u) \beta(u) A(u) d u
$$

The corresponding change in occupied surface area is found by multiplying Equation 3.12 with $a_{0}$ the area occupied by adsorbed polymer:

$$
a_{0} d w=k a_{0} C_{b}(u) \beta(u) A(u) d u
$$

Equation 3.13

Integrating both sides of Equation 3.13 over all possible values of $u$ gives the total area of interface covered with adsorbed polymer:

$$
a_{0} \int_{0}^{1} d w=k a_{0} \int_{0}^{1} C_{b}(u) \beta(u) A(u) d u
$$

The corresponding fraction of free interfacial area at time $t$ is:

$$
\beta(t)=1-\frac{k a_{0} \int_{0}^{t} C_{b}(u) \beta(u) A(u) d u}{A(t)}
$$


In our experiments the total interfacial area was given by the following equation where $A_{0}, A_{l}, \mathrm{f}$ and $\phi$ are known constants:

$$
A(t)=A_{0}+A_{1} \cos (2 \pi f t+\phi)
$$

Equation 3.16

Substituting Equation 3.16 into Equation 3.15 and assuming that the concentration of dissolved polymer $C_{b}(u)$ is constant, gives the following expression for the fraction of uncovered interface:

$$
\beta(t)=1-\frac{k a_{0} C_{b} \int_{0}^{t} \beta(u)\left(A_{0}+A_{1} \cos (2 \pi f u+\phi)\right) d u}{A_{0}+A_{1} \cos (2 \pi f t+\phi)}
$$

Taking the first derivative of the both sides of Equation 3.17 and solving for $\beta(t)$ gives:

$$
\frac{d \beta}{d t}-\frac{2 \pi A_{1} f \sin (2 \pi f t+\phi)-k a_{0} C_{b}\left(A_{0}+A_{1} \cos (2 \pi f t+\phi)\right)}{A_{0}+A_{1} \cos (2 \pi f t+\phi)} \beta=-\frac{2 \pi A_{1} f \sin (2 \pi f t+\phi)}{A_{0}+A_{1} \cos (2 \pi f t+\phi)}
$$

Equation 3.18

This is an ordinary linear first-order differential equation with the boundary condition $\beta(0)=1$. The solution for Equation 3.18 is:

$$
\beta(t)=\frac{A_{1} \cos (2 \pi f t+\phi)+\frac{k C_{b} a_{0} A_{1}}{2 \pi f} \sin (2 \pi f t+\phi)}{\left(1+\left(\frac{k C_{b} a_{0}}{2 \pi f}\right)^{2}\right)\left(A_{0}+A_{1} \cos (2 \pi f t+\phi)\right)}+
$$




$$
\frac{A_{0}+A_{1} \cos (\phi)-\frac{A_{1} \cos (\phi)+\frac{k C_{b} a_{0} A_{1}}{2 \pi g} \sin (\phi)}{1+\left(\frac{k C_{b} a_{0}}{2 \pi g}\right)^{2}}}{\left(A_{0}+A_{1} \cos (2 \pi f t+\phi)\right)} e^{-k C_{b} a_{0} t}
$$

\section{Equation 3.19}

In summary, Equation 3.19 gives the desired expression for $\beta$, which we used with Equation 3.11 to calculate the concentration of adsorbed polymer. The model involves two adjustable parameters: the adsorption rate constant, $k$ and the initial occupied area of an adsorbed polymer chain, $a_{0}$. All other parameters are known from the experiments. The concentrations of adsorbed polymer were converted to the corresponding surface tensions using Equation 3.7. See Appendix 3.1 for more details of the model derivation. 


\subsection{Results}

\subsubsection{Modeling Static Pendent Drop (SPD) Surface Tension Kinetics}

Surface tensions of aqueous PNIPAM solutions were determined from the pendent drop shapes as functions of drop age. Details of the experimental methods have been given previously [1]. Shown in Figure 3. 2 are surface tension versus time curves for five polymer concentrations at $25{ }^{\circ} \mathrm{C}$. The numbers beside the curves give the initial polymer concentration in ppm. The highest polymer concentration showed the most rapid decrease in surface tension.

The solid lines in Figure 3. 2 show the predictions of the Langmuir kinetics model coupled with the $\Gamma$-to- $\gamma$ transformation based on self-consistent field calculations (see Equation 3.8). The model was fitted to the $40 \mathrm{ppm}$ data giving $k_{1}=8.5 \times 10^{-4} \mathrm{~s}^{-1}$. The other model parameters were fixed: $\theta_{0}=0$ (i.e. a polymer-free initial surface); $k_{2} / k_{1}=0.001$ (i.e. essentially no desorption); and, $\Gamma_{\max }=0.9 \mathrm{mg} / \mathrm{m}^{2}$. The $n_{0}$ values (see Equation 3.1) were calculated from the polymer concentrations and the experimentally determined drop area together with $\Gamma_{\max }$. 


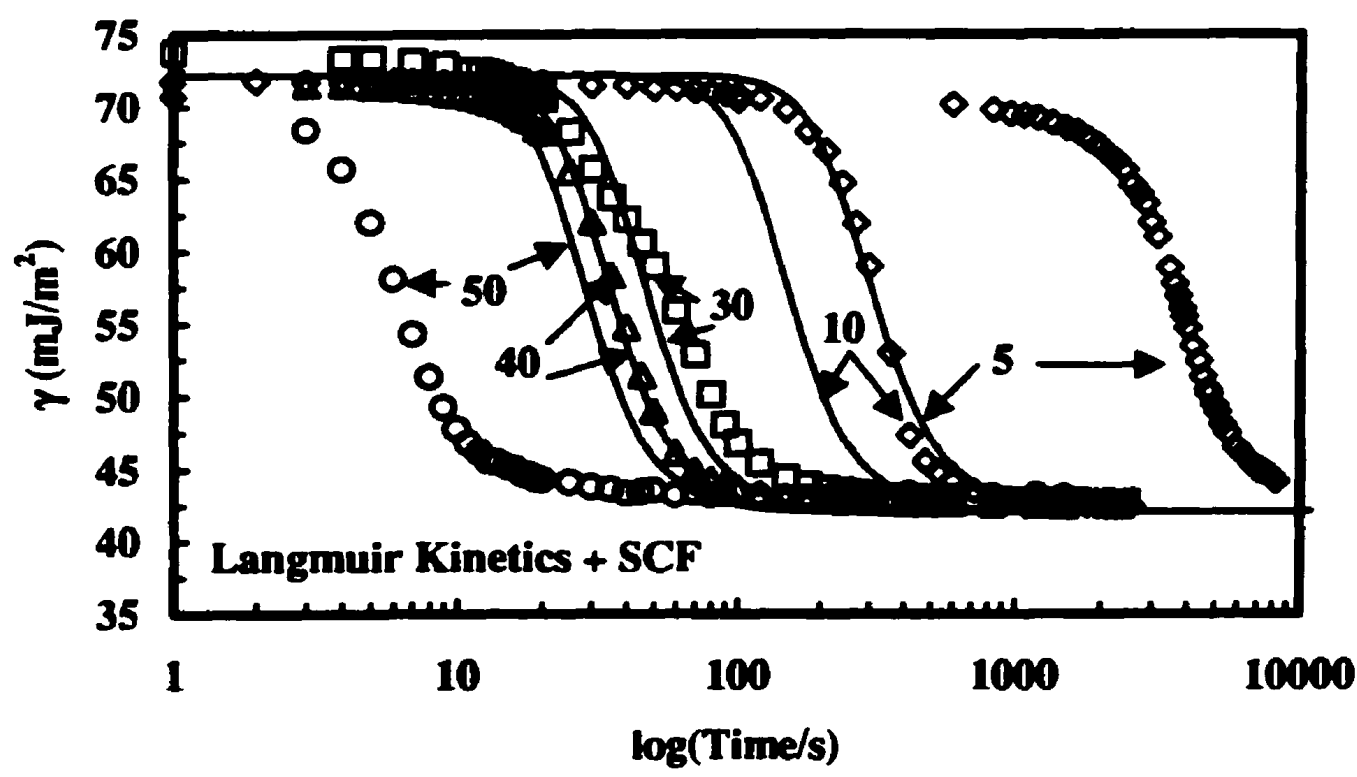

Figure 3. 2 Surface tension versus time for poly( $N$-isopropylacrylamide). The numbers besides the curves indicate the polymer concentration in $\mathrm{mg} / \mathrm{L}$ (ppm). The solid lines were computed using the Langmuir kinetics model together with $\Gamma$-to- $\gamma$ transformation from SCF.

The model was fitted to the $\mathbf{4 0} \mathrm{ppm}$ data and the resulting fit shown in Figure 3. 2 was excellent. In all cases the slopes of the predicted curves were close to the experimental results. The maximum slope of the $40 \mathrm{ppm}$ experimental curve was $-0.66 \mathrm{~mJ} \cdot \mathrm{m}^{-2} \cdot \mathrm{s}^{-1}$, which compares well with the model prediction of $-0.62 \mathrm{~mJ} \cdot \mathrm{m}^{-2} \cdot \mathrm{s}^{-1}$. On the other hand, the model did a poor job of predicting the times corresponding to the maximum rate of change in surface tension. For the lowest three concentrations the predicted drop in surface tension occurred before the corresponding experimental times with the biggest discrepancy corresponding to the lowest polymer concentration. By contrast, for the $50 \mathrm{ppm}$ calculation, the time corresponding to the midpoint of the curve was 
over predicted by an order of magnitude. Note that the computed curve on top of the 10 ppm data corresponds to the 5 ppm data.

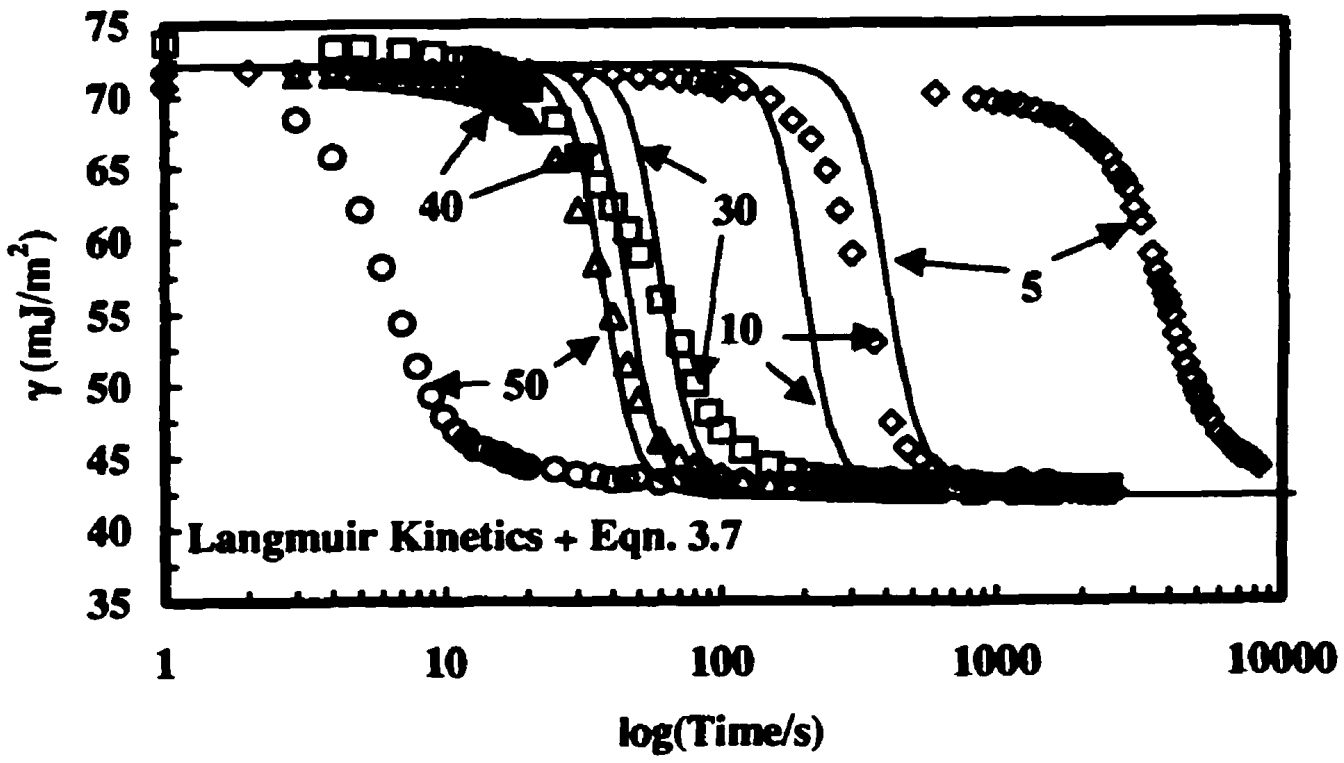

Figure 3. 3 Surface tension versus time curves computed with the Langmuir kinetics model in conjunction with the $\Gamma$-to- $\gamma$ transformation based on Kawaguchi's data (Equation 3.7).

The results of the Langmuir kinetics model were also converted to surface tension versus time curves using interpolated data (Equation 3.7). The computed results are compared with data in Figure 3. 3. Compared with the calculations in Figure 3. 2, the computed curves are shifted to longer times and the slopes of the computed curves are much greater than the previous calculations and the experimental slopes. For example, the maximum slope of the computed $40 \mathrm{ppm}$ curve in Figure 3. 3 was $-1.09 \mathrm{~mJ} \cdot \mathrm{m}^{-2} \cdot \mathrm{s}^{-1}$ which is nearly twice the experimental value (i.e. $\left.-0.66 \mathrm{~mJ} \cdot \mathrm{m}^{-2} \cdot \mathrm{s}^{-1}\right)$. 


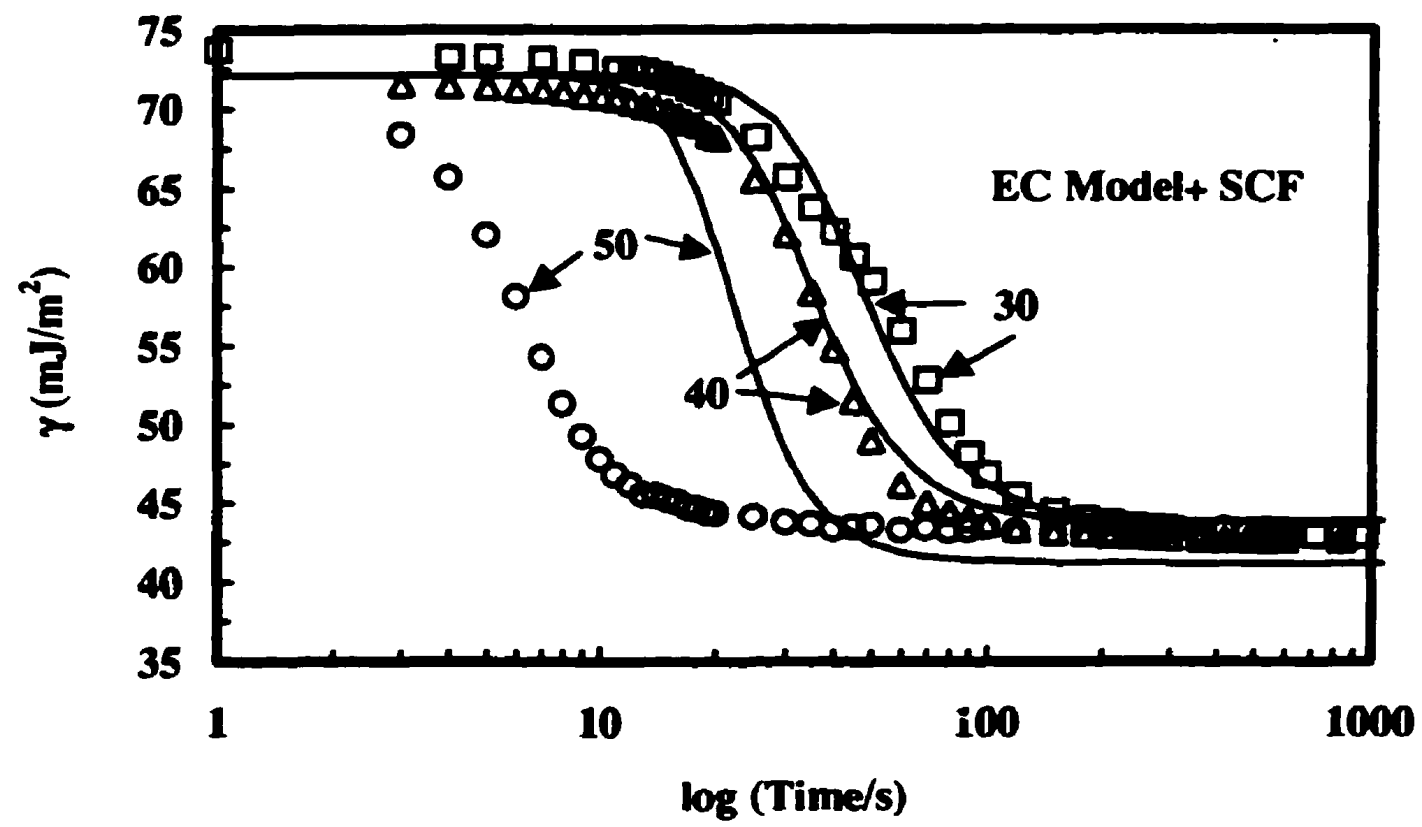

Figure 3. 4 Surface tension versus time curves computed with the EC model in conjunction with the $\Gamma$-to- $\gamma$ from self-consistent field calculations.

The Langmuir kinetics model is essentially a two parameter model $\left(k_{1}\right.$ and $\Gamma_{\max }$ ) in the absence of desorption. In an effort to improve the agreement between theory and experiment we employed the EC four parameter model. In addition to a rate constant, $k$ and $a_{0}$, the EC model has two more parameters $\alpha$ and $\tau_{s}$ which account for the spreading of adsorbed polymer. Figure 3. 4 compares the predictions of the EC model with our experimental data. The two lowest concentrations are not included because our version of the EC model does not account for the depletion of polymer from solution due to adsorption - this effect is not significant with high initial polymer concentrations (see Appendix 2.6). The parameters for the EC curves were $a_{0}=1 \mathrm{~m}^{2} / \mathrm{mg}, \alpha=0.3 \mathrm{~m}^{2} / \mathrm{mg}$ meaning the 
adsorbed polymer molecule can expand to a maximum of about $30 \%$ of its initial occupied area. $\tau_{s}$ was $10 \mathrm{~s}$ and $k$ was $5.5 \times 10^{-7} \mathrm{~m} / \mathrm{s}$. Inclusion of polymer reconformation had only a slight effect on the maximum slopes of the surface tension versus time curves. For example, the maximum slope of the computed $\mathbf{4 0}$ ppm curve was $-0.60 \mathrm{~mJ} \cdot \mathrm{m}^{-2} \cdot \mathrm{s}^{-1}$ compared with $-0.62 \mathrm{~mJ} \cdot \mathrm{m}^{-2} \cdot \mathrm{s}^{-1}$ for the Langmuir kinetics model.

In both the Langmuir and EC modeling it was not possible to change significantly the maximum slopes of the curves without also changing the final surface tension. The only way to lower the maximum slope significantly was to lower also the maximum amount of adsorbed polymer, which, in turn, gave too high a final surface tension.

The major difficulty in modeling the static pendent drop data was shifting the computed curves along the log time axis to correspond to the experimental data. Comparison of Figure 3. 2 with Figure 3. 4 showed that the EC model offered no advantage over the Langmuir kinetics model.

In summary, both the Langmuir kinetics model and the EC model predicted the slopes of the experimental dynamic surface tension curves when coupled with the SCF $\Gamma$-to- $\gamma$ transformation. On the other hand, neither model coupled to either Equation 3.7 or Equation 3.8 could predict the times corresponding to the maximum rates of change of surface tension. 


\subsubsection{Modeling Oscillating Pendent Drop (OPD) Surface Tension Kinetics}

In the OPD experiments polymer solution was pumped into and out of a pendent drop at a constant volumetric flow. The corresponding change in drop area, which is calculated from the drop shape, was fitted to a cosine function. Figure 3. 5 shows an example of experimentally determined surface areas together with the empirical equation used to fit the data.

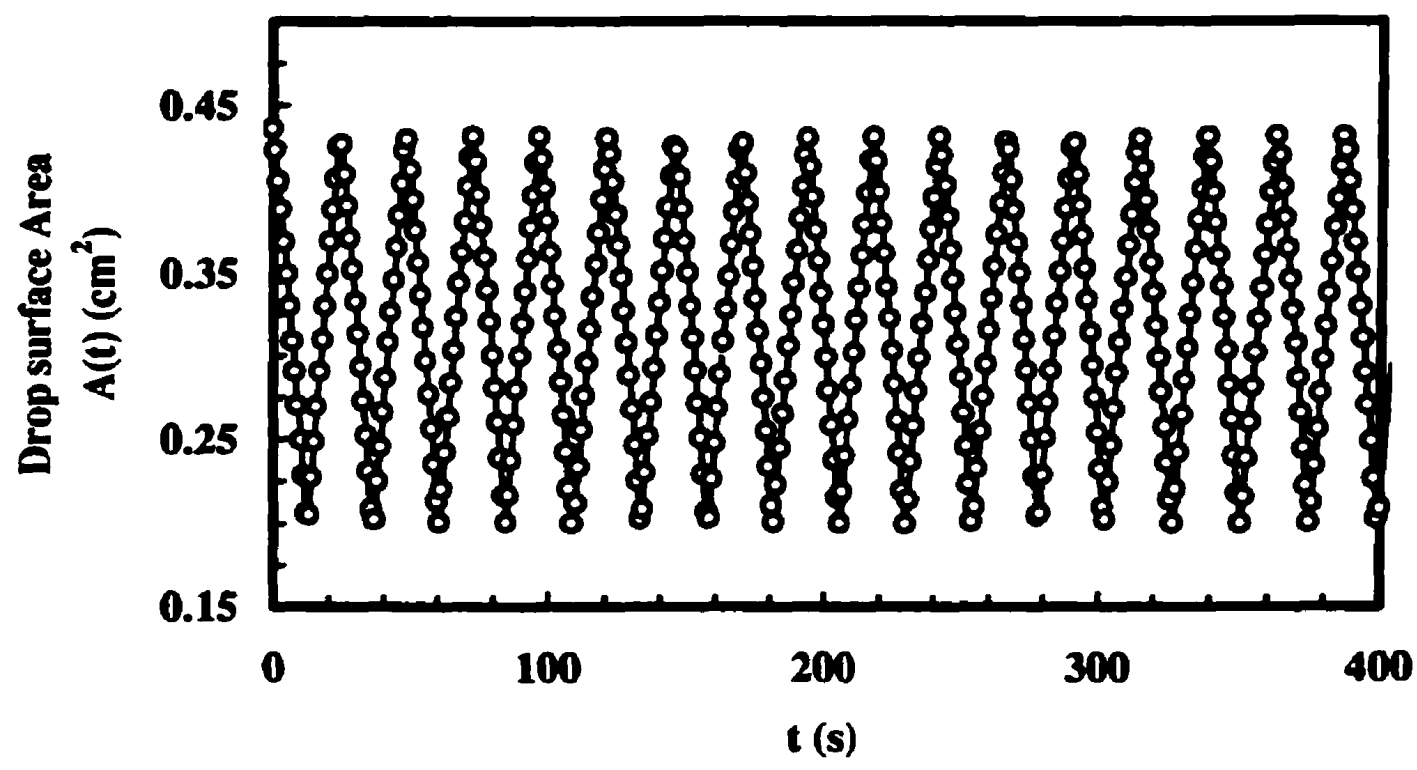

Figure 3.5 An example of the experimental control of the pendent drop area. The circles are experimental values of the drop surface area for L-6B $10 \mathrm{ppm}$ solution in a oscillating period of $24.2 \mathrm{~s}$, the solid line is the curve of the corresponding mathematical description $\left(R^{2}=0.9825\right)$ :

$$
A(t)=0.3204+0.0978\left(\cos \left(\frac{2 \pi t}{24.2}-0.0918\right)\right)
$$

Equation 3.11 was used with Equation 3.19 to model OPD experiments. 
Figure 3. 6 shows the fraction of free surface area $(\beta)$ and the surface concentration $(\Pi$ ) for three polymer concentrations. The values of the parameters used in the calculations are given in the figure caption. As expected, the more concentrated solutions gave faster polymer adsorption. The influence of the surface area oscillations is significant for all three plots. Negative $\beta$ values were calculated for the 50 and $10 \mathrm{ppm}$ curves. This situation occurred when a drop saturated with polymer had its surface area decreased. Physically this corresponds to the collapse of an insoluble monolayer when highly compressed on a Langmuir trough. Kwok [29] reported monolayer collapse in oscillating pendent drop experiments with insoluble octadecanol monolayer film.

The dynamic surface tension curves shown in Figure 3. 6 were computed from the surface concentrations using Equation 3.7. The $50 \mathrm{ppm}$ curve quickly approached steady state oscillations whereas the $5 \mathrm{ppm}$ curve did not reach steady state in the time scale of the calculation. Note the product $k C_{b}$ occurs in all the equations, so that changing the rate constant has the same effect as changing the polymer concentration.

The dynamic surface tensions of PNIPAM aqueous solutions were measured at oscillation frequency $(f)$ of $0.04,0.03$ and $0.02 \mathrm{~Hz}$ for $10 \mathrm{ppm}$ solution and at $0.03 \mathrm{~Hz}$ for $50 \mathrm{ppm}$ - the experimental details are given a previous paper [1]. These results are now compared with the predictions of our model. 

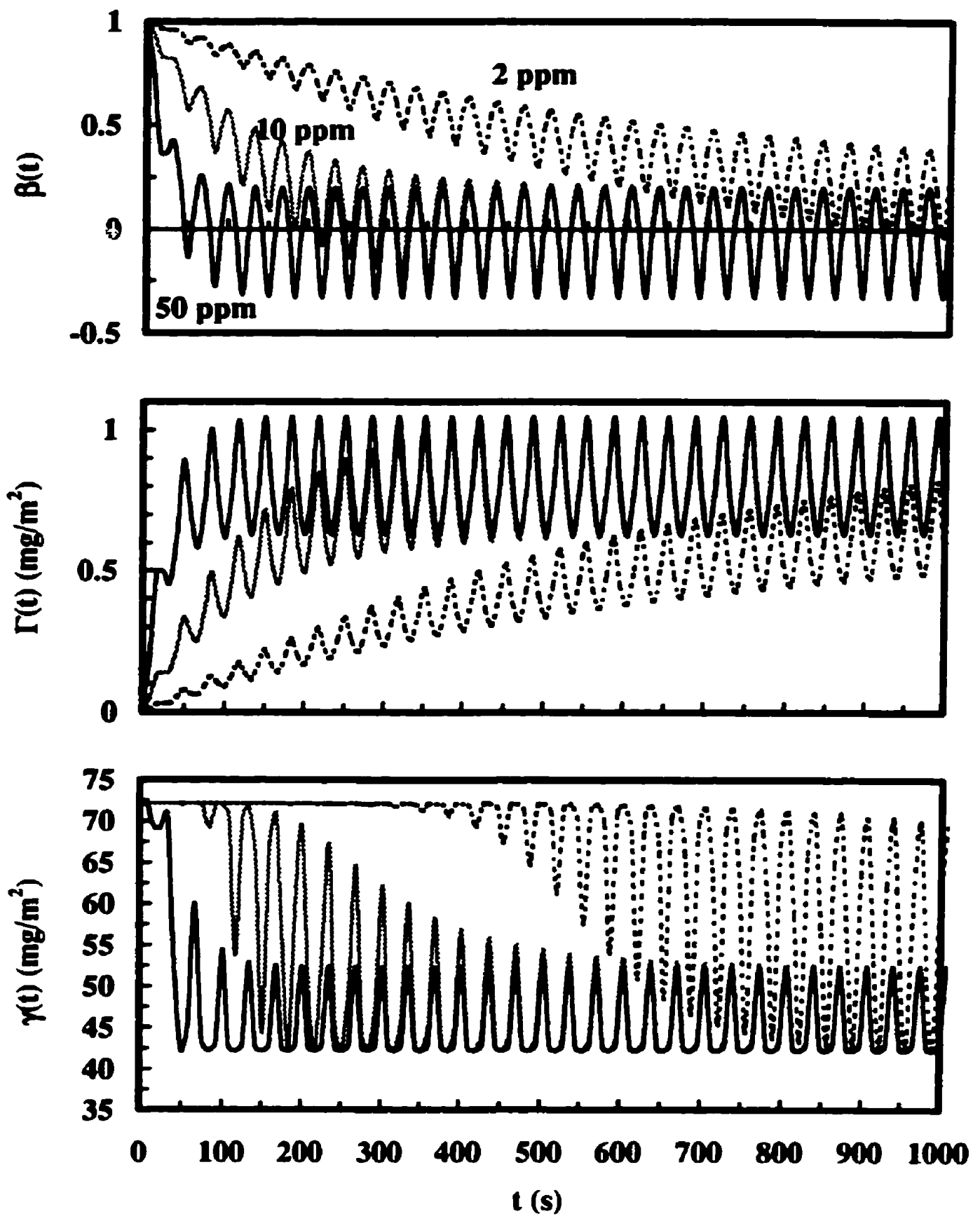

Figure 3.6 The $\mathrm{C}_{\mathrm{b}}$ dependence of $\beta(t), \Gamma(t)$ and $\chi(t)$ for a moving interface as predicted by the model. $k=6.0 \times 10^{-7} \mathrm{~m} / \mathrm{s}, a_{0}=1.27 \mathrm{~m}^{2} / \mathrm{mg}, A_{0}=0.3226 \mathrm{~cm}^{2}, A_{1}=0.0818 \mathrm{~cm}^{2}, f=$ $0.03 \mathrm{~Hz}$ (or $P=33.6 \mathrm{~s}$ ), $\phi=0.2276$ radian. 

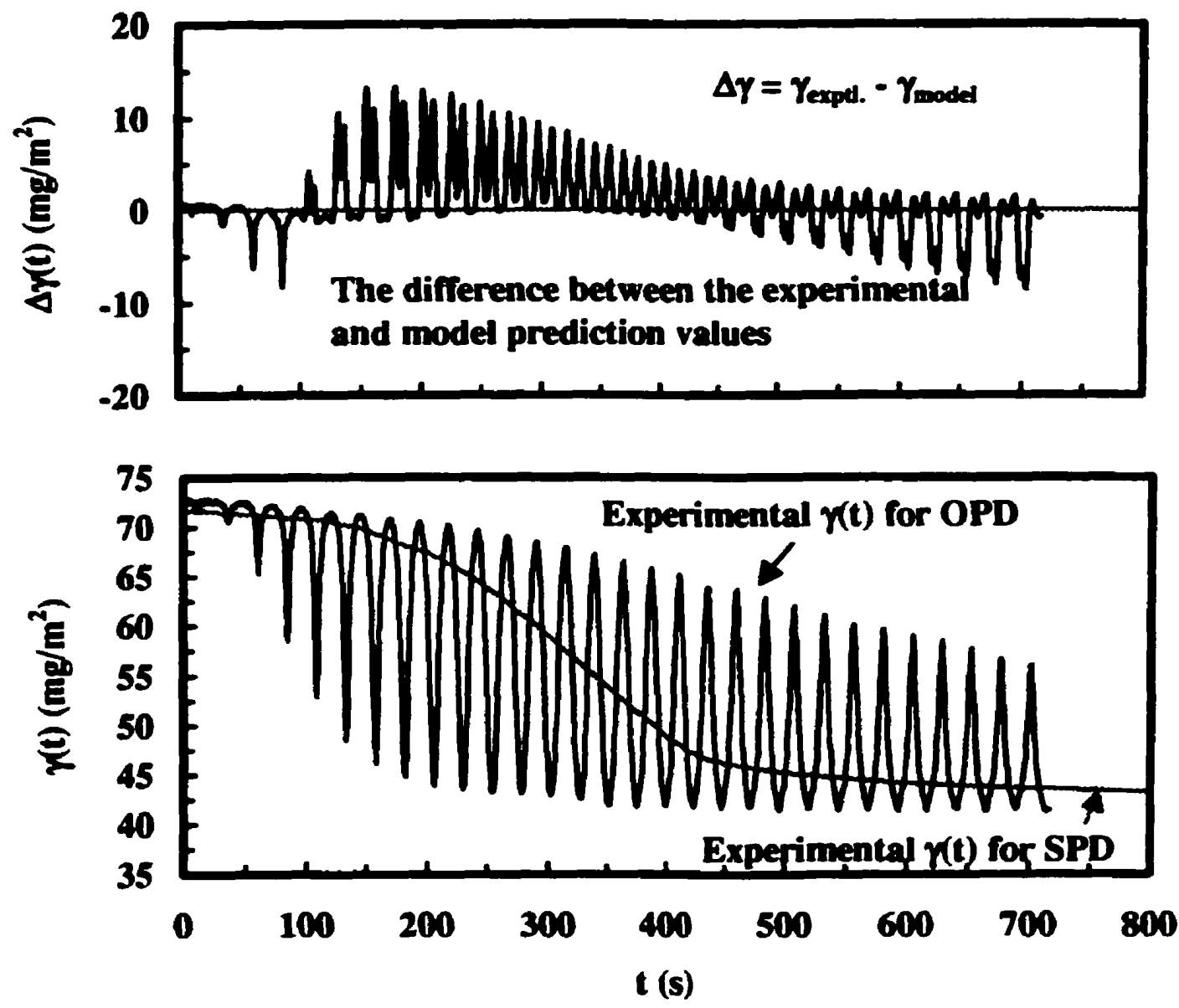

Figure 3.7 The lower plot shows experimental results from OPD and SPD experiments. The upper curve shows $\Delta \gamma=\gamma_{\text {model }}-\gamma_{\text {copp }}$. Parameters were $\alpha=1.9 ; k=6 \times 10^{\circ}$ ${ }^{7} \mathrm{~m} / \mathrm{s} ; \quad C_{b}=10 \mathrm{ppm} ; a_{0}=1.3482 \mathrm{~m}^{2} / \mathrm{mg} ; f=0.04 \mathrm{~Hz}$ (or $P=24.2 \mathrm{~s}$ ); $A_{0}=0.3204 \mathrm{~cm}^{2} ;$ $A_{1}=0.0978 \mathrm{~cm}^{2}$ and $\infty=0.0664$ radian.

The lower part of Figure 3. 7 shows experimental surface tension versus time curves for both SPD and OPD experiments. As reponted previously the OPD results showed large oscillations in surface tension in response to the changes in drop area. Furthermore, the rate of surface tension lowering was greater in the oscillating experiments than in the SPD results, presumably reflecting the 
influence of liquid pumping on mass transport of polymer to the surface. The model results are shown in the top part of Figure 3. 7 and are expressed as the difference between experimental and calculated surface tensions. At early stages (200 s) the model over-predicted the minimum surface tension peaks by as much as $15 \mathrm{~mJ} / \mathrm{m}^{2}$. Whereas at long times the model predicted larger increases of surface tension with increasing drop area. In other words the polymer seemed to rearrange with drop expansion to partially compensate for increased surface area giving a lower surface tension than expected.

More data are shown in the bottom half of Figure 3.8 and in Figure 3.9. Different frequencies were used for the three sets of experiments in Figures 3.7 to 3.9. However, the major difference was the extent by which the surface areas were changed. The $\alpha$ values shown in the figure captions are the maximum drop areas divided by the corresponding minimum values. The larger $\alpha$ values induced larger swings in surface tension at long times as well as giving a slower approach to steady state. The corresponding model calculations used the same $\mathbf{k}$ value and slightly different $a_{0}$ in the three figures. The model had the most difficulty at the early times when rates of polymer adsorption were high. However, considering the complexity of the experiments and that the model had only two (i.e. $k$ and $a_{o}$ ) adjustable parameters, the calculations seemed surprisingly good. 

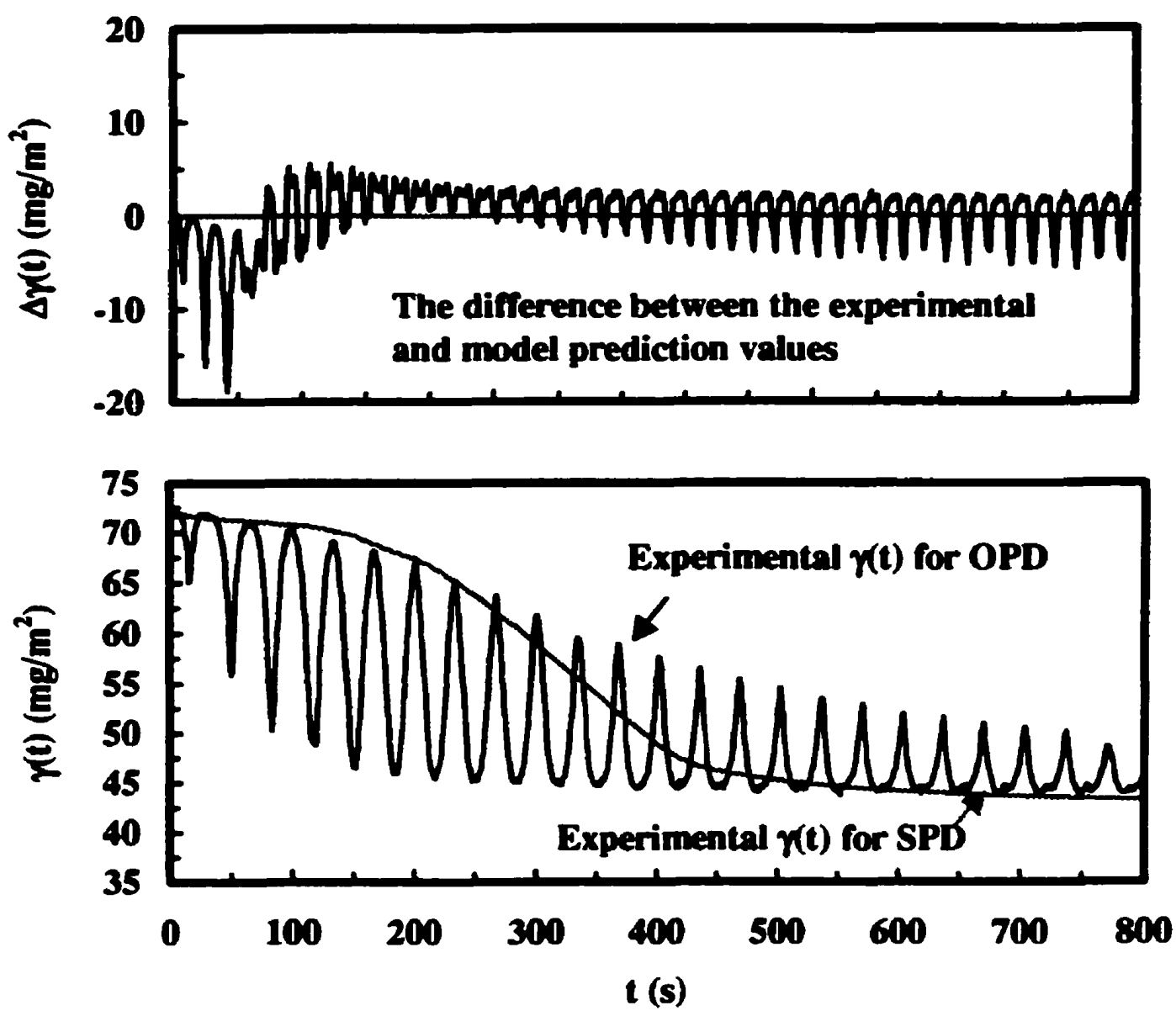

Figure 3.8 Comparison of experimental results with the model predictions. $\alpha=$ 1.68; $k=6 \times 10^{-7} \mathrm{~m} / \mathrm{s} ; C_{b}=10 \mathrm{ppm} ; a_{0}=1.25 \mathrm{~m}^{2} / \mathrm{mg} ; f=0.03 \mathrm{~Hz}$ (or $P=33.6 \mathrm{~s}$ ); $A_{0}=0.3226 \mathrm{~cm}^{2}$; $A_{1}=0.0818 \mathrm{~cm}^{2}$ and $\$ 0.2276$ radian. 

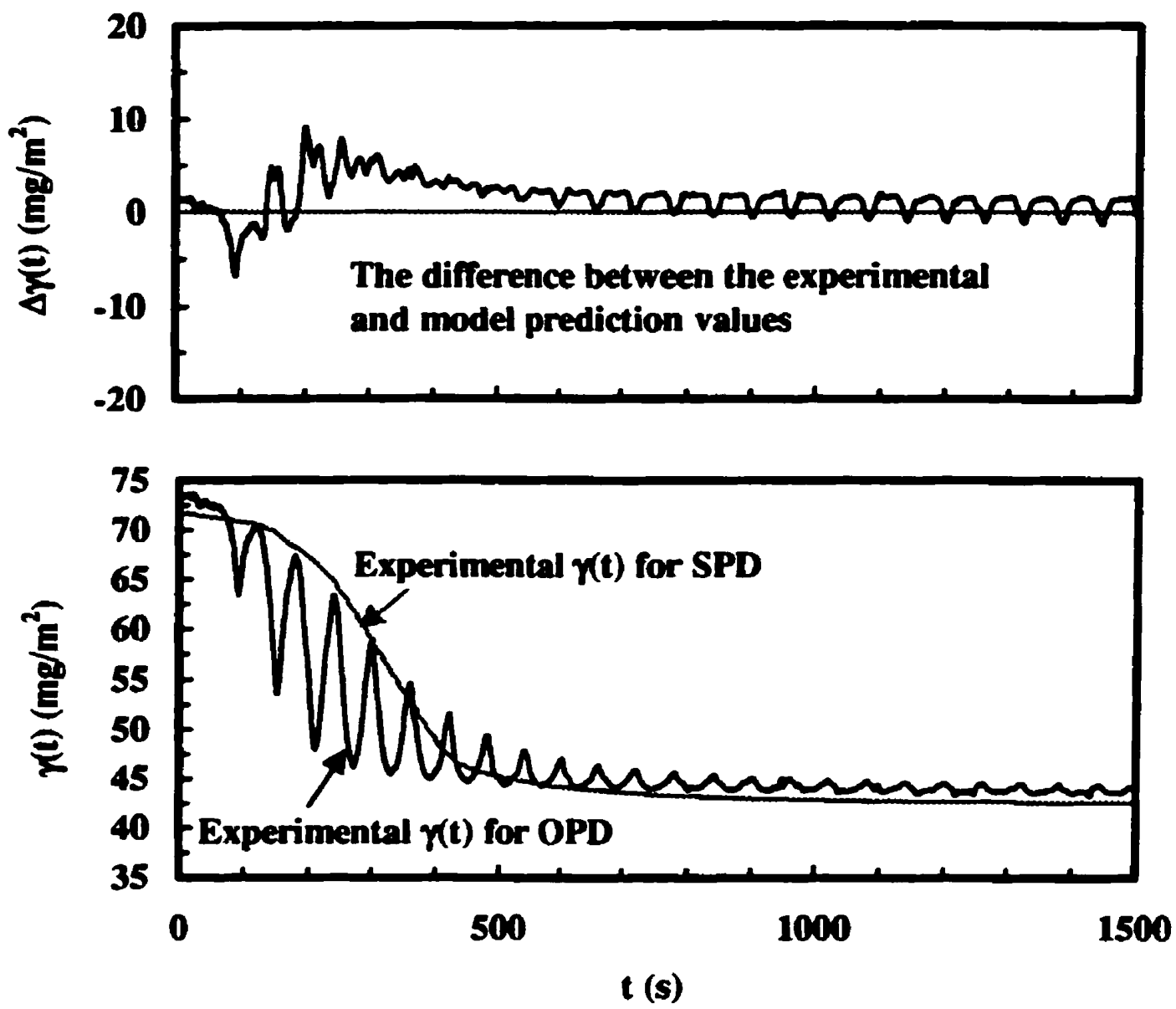

Figure 3.9 Comparison of experimental results with the model predictions. $\alpha=$ $1.39 ; k=6 \times 10^{-7} \mathrm{~m} / \mathrm{s} ; C_{b}=10 \mathrm{ppm} ; a_{0}=1.24 \mathrm{~m}^{2} / \mathrm{mg}, f=0.02 \mathrm{~Hz}$ (or $P=60.1 \mathrm{~s}$ ); $A_{0}=0.3139 \mathrm{~cm}^{2}$; $A_{1}=0.0507 \mathrm{~cm}^{2}$ and $=0.1076$ radian. 

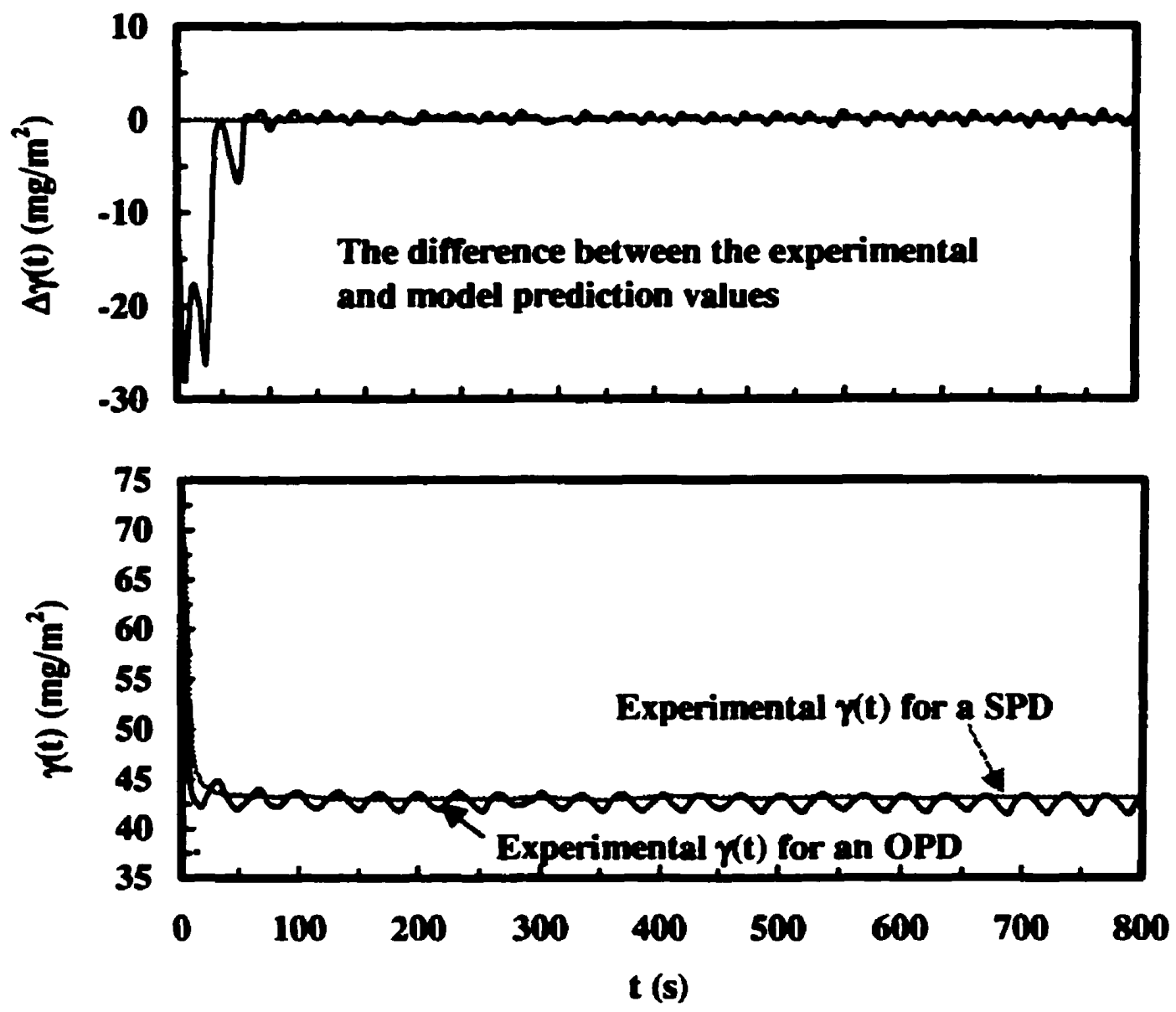

Figure 3.10 Comparison of experimental results with the model predictions. $k=6 \times 10^{-}$ ${ }^{7} \mathrm{~m} / \mathrm{s} ; C_{b}=50 \mathrm{ppm} ; a_{b}=1.00 \mathrm{~m}^{2} / \mathrm{mg} ; f=0.03 \mathrm{~Hz}$ (or $P=33.6 \mathrm{~s}$ ); $A_{b}=0.3226 \mathrm{~cm}^{2} ; A_{l}=0.0818 \mathrm{~cm}^{2}$ and $\phi=0.2276$ radian. 


\subsection{Discussion}

With surfactants it is generally possible to generate experimental surface tension versus equilibrium solution concentration plots which can be analyzed by the Gibbs adsorption isotherm By contrast, polymer adsorption at the air/water interface shows high affinity behavior, which means that it is usually not experimentally possible to obtain surface tension as a sensitive function of polymer concentration in the bulk phase. Indeed, we have shown that for poly $(N$ isopropylacrylamide) the surface tensions obtained at long times are rather insensitive to polymer concentration in the bulk phase and molecular weight [1]. On the other hand, polymer solutions display a rich variety of surface tension kinetics. In this work we have attempted to model dynamic surface tension results with polymer adsorption theories.

The modeling herein is based on the assumption that there is a unique relationship between the mass concentration of adsorbed polymer, $\Gamma$, and the surface tension (e.g. we assumed either Equation 3.7 or Equation 3.8). If surface tension is dictated by only the polymer segments attaching to the interface, then this assumption is equivalent to assuming the number of bound segments is a unique function of $\Gamma$ for polymer adsorption onto a solid. This can only be correct if the extent of reconfiguration is independent of the concentration of polymer in the bulk phase. One might expect that a polymer molecule adsorbed from very low concentration solutions would spread to give more 
adsorbed segments with time and a corresponding decrease in surface tension. The steady-state experimental surface tension results were independent of polymer concentration in bulk phase suggesting that the final configurations were not strong functions of bulk concentration.

The static pendent drop experiments should be the easiest to model. After rather ill defined initial boundary conditions (i.e. what is time zero and the corresponding initial concentration of adsorbed polymer during pendent drop formation), polymer transport in small quiescent drops should be dominated by diffusion. Good fits were obtained to the slopes of the curves in Figure 3. 2 and Figure 3. 4. Furthermore, fitting of the slopes was sensitive to details of the $\Gamma$-to$\gamma$ transformation indicating that this modeling is a good test for these relationships.

Our modeling was unsuccessful at predicting the time scale for surface tension lowering. Figure 3. 2 and Figure 3. 4 show that models fitted to the 40 ppm results overestimated the time for maximum surface tension decrease by an order of magnitude for the $50 \mathrm{ppm}$ polymer results. In other words, concentrated polymer solutions lower surface tension much more quickly than do dilute ones compared with our expectations from a diffusion-controlled process. Therefore, we conclude that the kinetics is influenced by other processes in addition to the diffusion-controlled transport of polymer to the interface. Furthermore, such processes must account for the fact that the final surface tensions were 
independent of polymer concentration in the bulk phase.

One possibility examined in this work was the expansion of adsorbed polymer competing with newly adsorbing polymer for free surface. We used a version of the Eijk and Cohen Stuart model [25]. This approach was rejected because if reconformation is significant, then polymer adsorbed from very low concentration solutions will expand to saturate the interface giving a relatively low mass concentration of adsorbed polymer and thus a high equilibrium surface tension.

A variation of reconfiguration might explain the experimental results. At high solution concentration, polymer adsorbs quickly to give a saturated interface and a low surface tension. By contrast at low concentration, adsorbed polymer reconfigures. However, instead of assuming that spread polymer blocks further adsorption, it is possible that additional polymer molecules approaching the interface could penetrate the adsorbed polymer layer to eventually give the same surface tension that was obtained for the concentrated polymer solution. Compared with a solid/solution interface, the air/water interface is mobile, enabling adsorbed segments to move laterally to allow other chains to adsorb at the interface. Such a process is analogous to people sitting on a park bench sliding over to allow a new arrival to sit down. Furthermore, this rearrangement step should be much slower than diffusion to a clean interface, thus explaining the slow kinetics for low polymer concentrations. 
The OPD data showed wild initial surface tension oscillations, which were poorly predicted by our simple model. Considering the complexity of the flows this is not surprising. At long times when mass transport to the interface was less important, uniform surface tension oscillations were observed. The predicted changes in surface tension due to the expansion of the interfacial area were greater than the observed amplitudes of the surface tension fluctuations. This suggests in 15 second time scale of the drop expansion, polymer loops and tails laid down upon newly formed surface during drop expansion and that the process was reversible.

In summary, attempts to model SPD and OPD experiments were only partially successful. More quantitative information about the relationships between the amount of adsorbed polymer, $\Gamma$, the configuration of adsorbed polymer and surface tension, $\gamma$, is required. Dynamic surface tension measurements coupled with ellipsometry or neutron reflectivity results could give more insight. Quantitative prediction of the amplitude of the steady-state surface tension oscillations is a challenging test of our understanding of the behavior of polymer at the air/water interface. 


\subsection{Conclusions}

1. The slopes of the calculated surface tension versus log time curves were sensitive to the assumed relationship between surface tension and surface concentration. Equation 7 based on self-consistent field calculations gave the best results.

2. Based on the observation that simple mass transport models could not predict the time scale for surface tension lowering over the range of polymer concentrations, it is proposed that a slow process involving the penetration of polymer chains through previously adsorbed chains contributes to the kinetics.

3. The major features of the oscillating pendent drop experiments were predicted by a two-parameter model. However, the experimental steady-state surface tensions oscillations were less than the model prediction, indicating the adsorbed polymer re-arranged to compensate for an expanding interface. 


\subsection{Acknowledgement}

We would like to thank Professor Cameron M. Crowe at McMaster University and Dr. HongJie Yang at Royal Bank of Canada for their help with the mathematical derivations, Professor W. Wilhelm Neumann and Dr. Daniel Yu Hing Kwok at University of Toronto for their help with the dynamic surface tension measurements. We thank the Canadian Natural Science and Engineering Research Council for financial support. 


\subsection{References}

1 Zhang, Ju and Pelton, R., "The Dynamic Behavior of Poly $(N$ isopropylacrylamide) at the Air/water Interface" accepted by Colloids and Surfaces.

2 Fleer, G. J.; Cohen Stuart, M. A.; Scheutjens, J. M. H.; Cosgrove, T.; Vincent, B., Polymers at Interfaces; Chapman \& Hall: London, 1993.

3 Shaaf, P. and Talbot, J., Phys. Rev. Lett. 62, 175 (1989).

4 Boyer, D.; Talbot, J.; Tarjus, G.; Van Tassel, P. and Viot, P., Phys. Rev. E., 49, 5525 (1994).

5 Van Tassel, P. R.; Talbot, J.; Tarjus, G. and Viot, P., Phys. Rev. E., 53, 785 (1996).

6 Van Tassel, P. R., J. Chem. Phys., 101, 7064 (1994).

7 Bagnal, R. D., J. Biomed. Mater. Res., 11, 947 (1977).

8 Graham, D. E. and Phillips, M. C., J. Colloid Interface Sci., 70, 415 (1979).

9 Benjamins, J.; de Feijter, J. A.; Evans, M. T. A.; Graham, D. E. and Phillips, M. C., Faraday Discuss. Chem. Soc., 59, 218 (1975).

10 Benjamins, J.; de Feijter J. A.; Evan, M. T. A.; Graham, D. E. and Phillips, M. C., Faraday Discuss. Chem. Soc., 59, 254 (1975).

11 Phillips, M. C. and Sparks, C. E., Ann. NY Acad. Sci., 348, 122 (1980).

12 Lankveld, J. M. G. and Lyklema, J., J. Colloid Interface Sci., 41, 454 (1972).

13 Hunter, J. R.; Carbonell, R. G. and Kilpatrick, P. K., J. Colloid Interface Sci., 137,462 (1990).

14 Furuno, T. and Sasabe, H., J. Colloid Interface Sci., 147, 225 (1991).

15 Van Tassel, P. R.; Viot, P. and Tarius, G., J. Chem. Phys., 106, 761 (1997).

16 Frantz, P. and Granick, S., Phys. Rev. Lett., 66, 899 (1991). 
17 Cosgrove, T; Prestige, C. A.; King, S. M. and Vincent, B, Langmuir, 8, 2206 (1992).

18 Cohen Stuar, M.A. and Fleer, G.J., Annu. Rev. Mater. Sci., 26, 463 (1996).

19 Meakin, P. and Jullien, R., Phys. Rev. A., 46, 2029 (1992).

20 Talbot, J. and Schaaf, P., Phys. Rev. A., 40, 422 (1989)

21 van de Ven, T.G.M., Colloidal Hydrodynamics, Academic Press, New York, 1989.

22 Lyklema, J., Fundamentals of Interface and Colloid Science, Vol.1, Chapter 6, Academic Press, New York, 1991.

23 Ward, A.F.H and Tordi, L., J. Chem. Phys., 63, 473 (1961).

24 van de Ven, T.G.M., Advances Colloid Interface Sci., 48, 121 (1994).

25 Van Eijk, M. C. P. and Cohen Stuart, M. A., Langmuir 13, 544 (1997).

26 Pefferkorn, E. and Elaissari, A., J. Colloid Interface Sci., 138, 187 (1990).

27 Kawaguchi, M.; Saito, W. and Kato, T., Macromolecules, 27, 5882 (1994).

28 G.J. Fleer, M.A. Cohen Stuart, J.M.H.M. Scheutjens, T. Cosgrove, and B. Vincent, Chapter 4 in Polymers at Interfaces, Chapman Hall, New York, 1993.

29 Kwok, D. Y., Master Thesis, University of Toronto, 1994. 


\section{Appendix 3.1 Modeling the Dynamic Surface Tension}

\section{Summary}

The modeling in this document is a modification of the model developed by Eijk and Cohen Stuar (the EC model) [1]. This modeling is to model the dynamic surface tension of PNIPAM aqueous solutions, which combine two steps: (1) modeling the surface concentration of adsorbed polymers, and (2) applying the empirical relationship between the surface tension and surface concentration (Equation A3.1.26, see Appendix 3.2). The approach to the solution of the surface concentration is through modeling the total adsorbed amount of polymers at the air/water interphase, not by modeling the surface concentration (as in the EC model). In such a way that both the bulk conc., the fraction of the free surface area and the changing total surface area $A(t)$ all contribute to the change of the amount of polymers adsorbed. For the constant surface area case (no oscillation), both sides of Equation A3.1.1 devided by the total drop surface area $A$, the model is reduced to the $E C$ model (with $J=k^{*} C_{b}$ and $\Gamma=w / A$ ).

\section{Symbols}

the length of the experimental time s

$\mathrm{u} \quad$ any time between 0 to $\mathrm{ts}$

w the total amount of macromolecules adsorbed at the air/water interface, $\mathbf{m g}$

$A(t)$ the interfacial area, which can be a constant $A$ (for the static pendant drop experiments) or a function of time (for the oscilating pendant drop experiments), $\mathrm{m}^{2}$

$k$ the adsorption rate constant, $\mathrm{m} / \mathrm{s}$ 


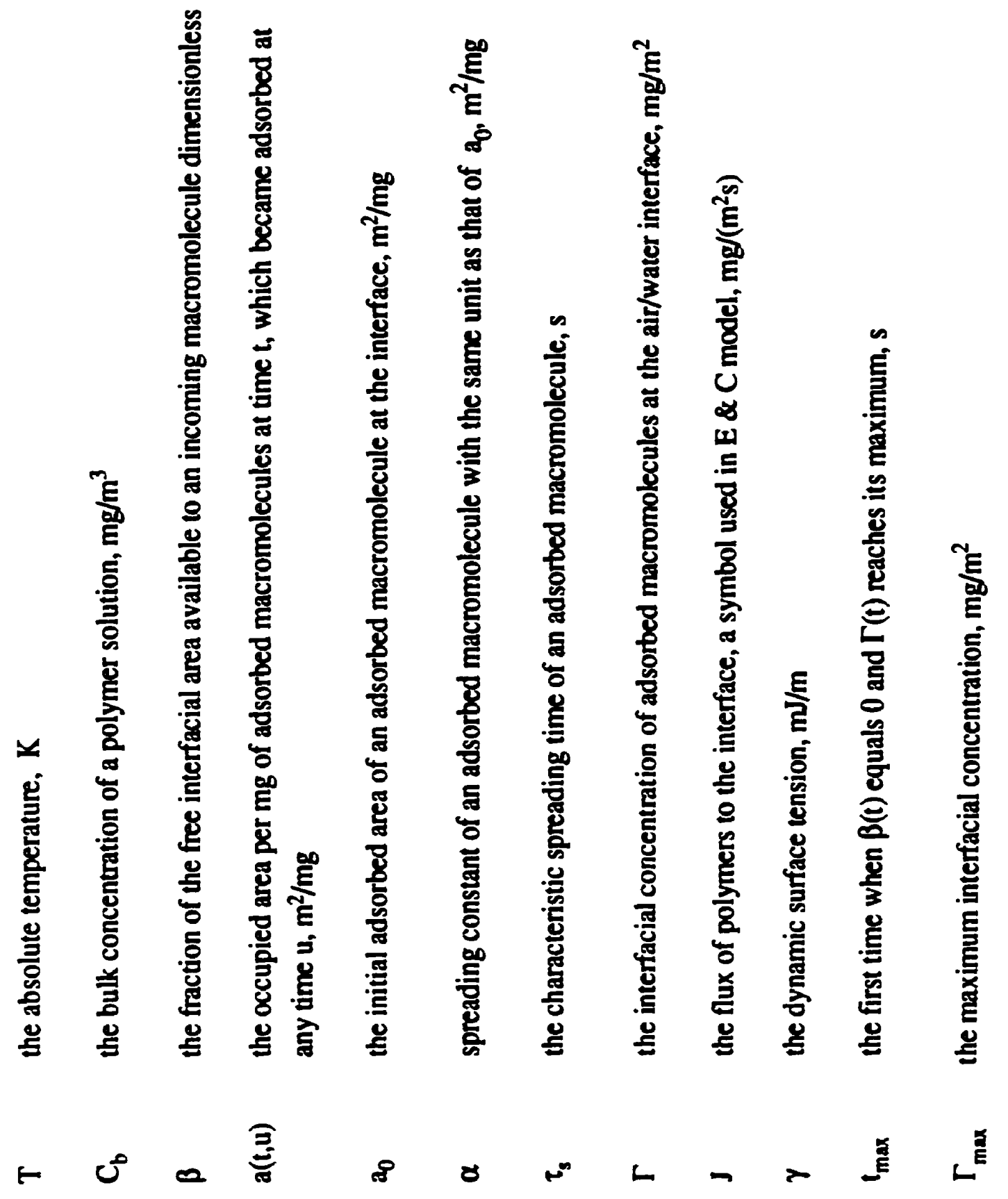


$A_{0}$ the mean of the changing area of an interface under a cosinoidal oscillation, $\mathrm{m}^{2}$

$A_{1}$ the oscillation amplitude of a cosinoidal oscillation of the changing interfacial area, $m^{2}$

P the period of the cosinoidal oscillation, $s$

$\phi \quad$ the phase of the cosinoidal oscillation, radian

\title{
Assumption 1
}

fraction

The adsorption rate of macromolecules to the air/water interface is proportional to the bulk concentration, the of the free interfacial area and the total interfacial area in a manner described by the following equation $[1,2]$

$$
\frac{d}{d t} w=k \cdot C_{b}(t) \cdot \beta(t) \cdot A(t) \quad \text { Equation A3.1.1 }
$$

\section{Assumption 2}

Macromolecules adsorb to the air/water interface with an initial area ao per mg of macromolecules. Once adsorbed, spread at the interface with time in the following manner [2]:

$$
a(t, u)=a_{0}+\alpha \cdot\left[1-\exp \left[\frac{-(t-u)}{\tau_{s}}\right]\right]
$$

\author{
Equation A3.1.2
}


I

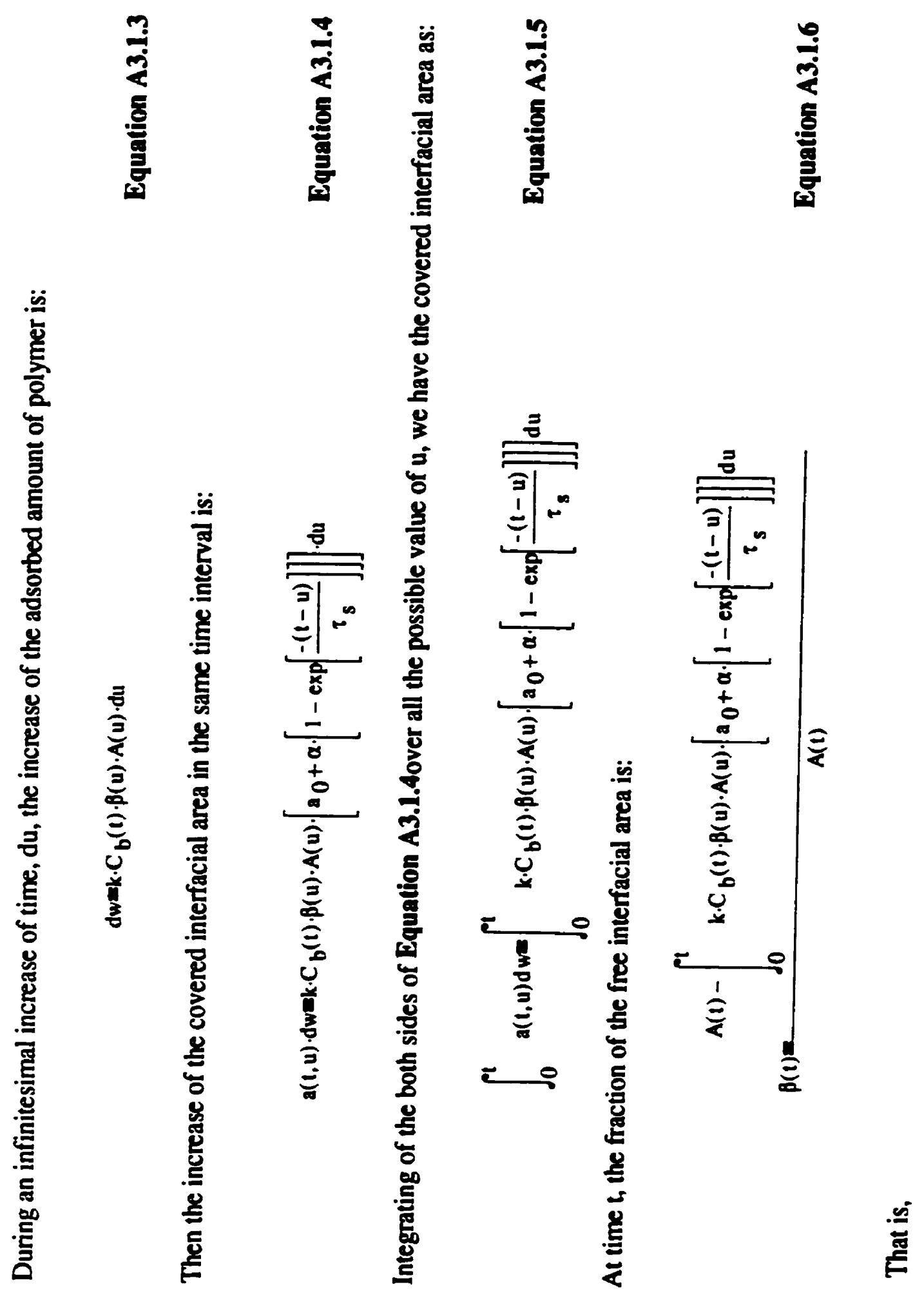


邑

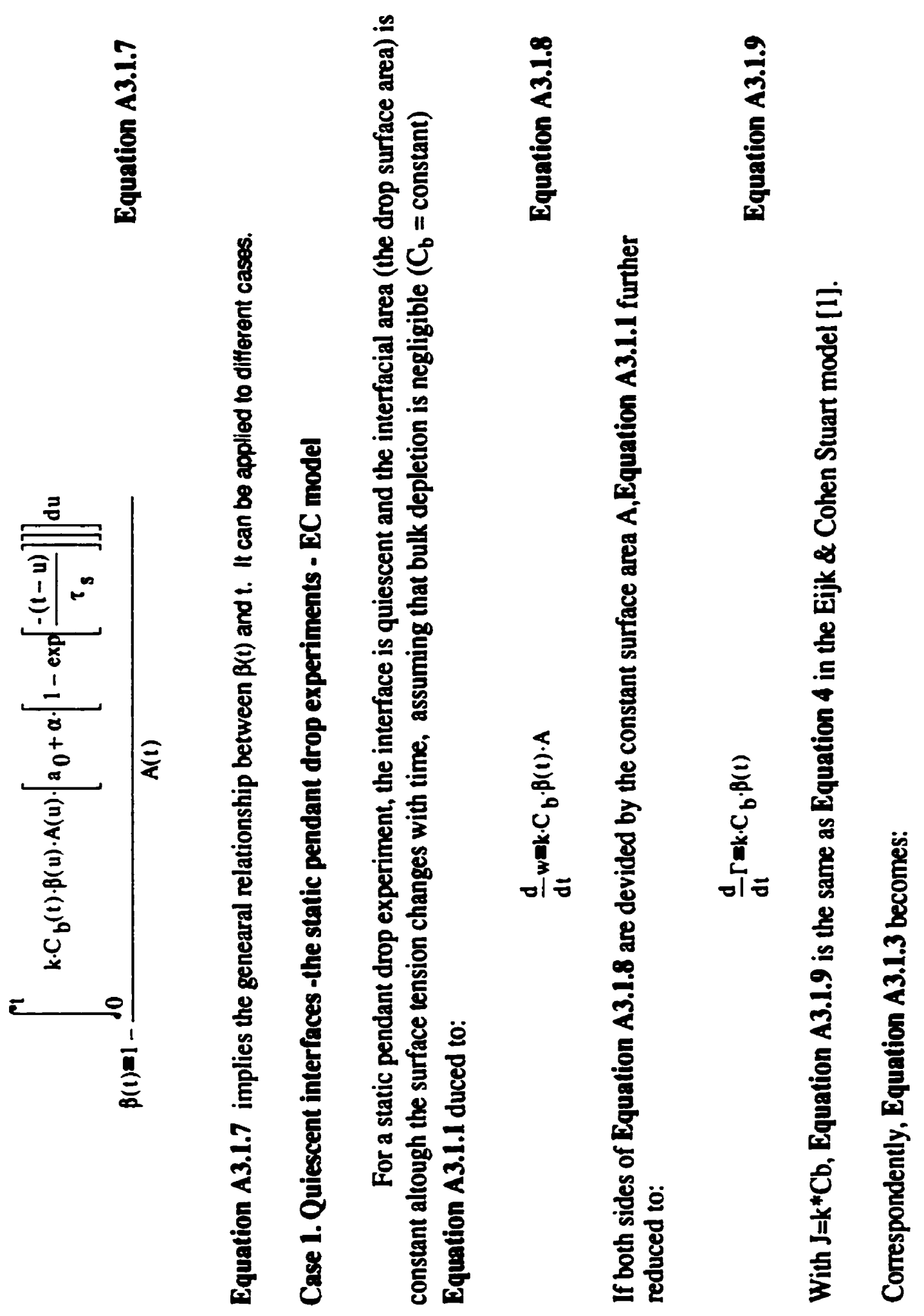


$\stackrel{\circ}{9}$

을
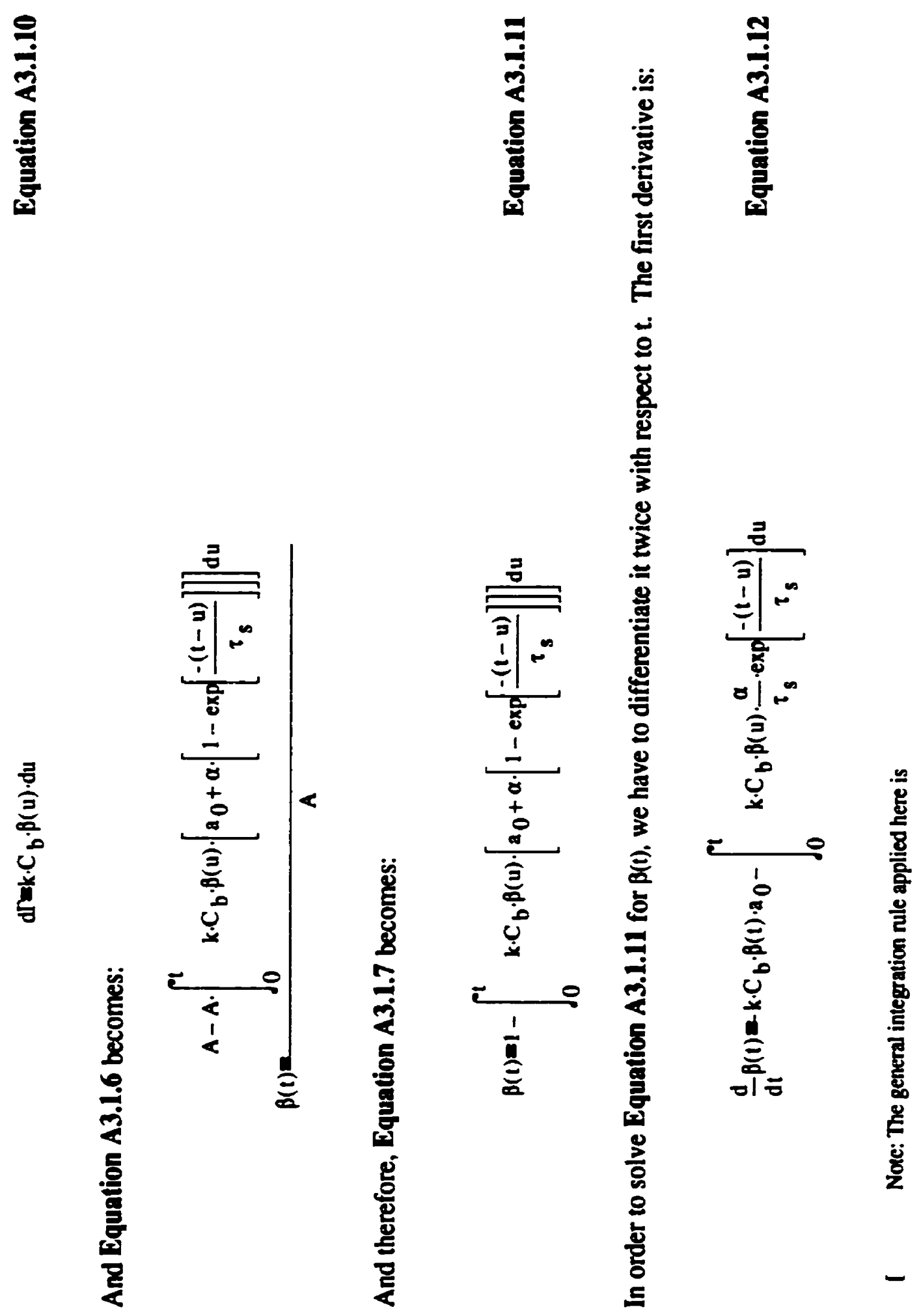


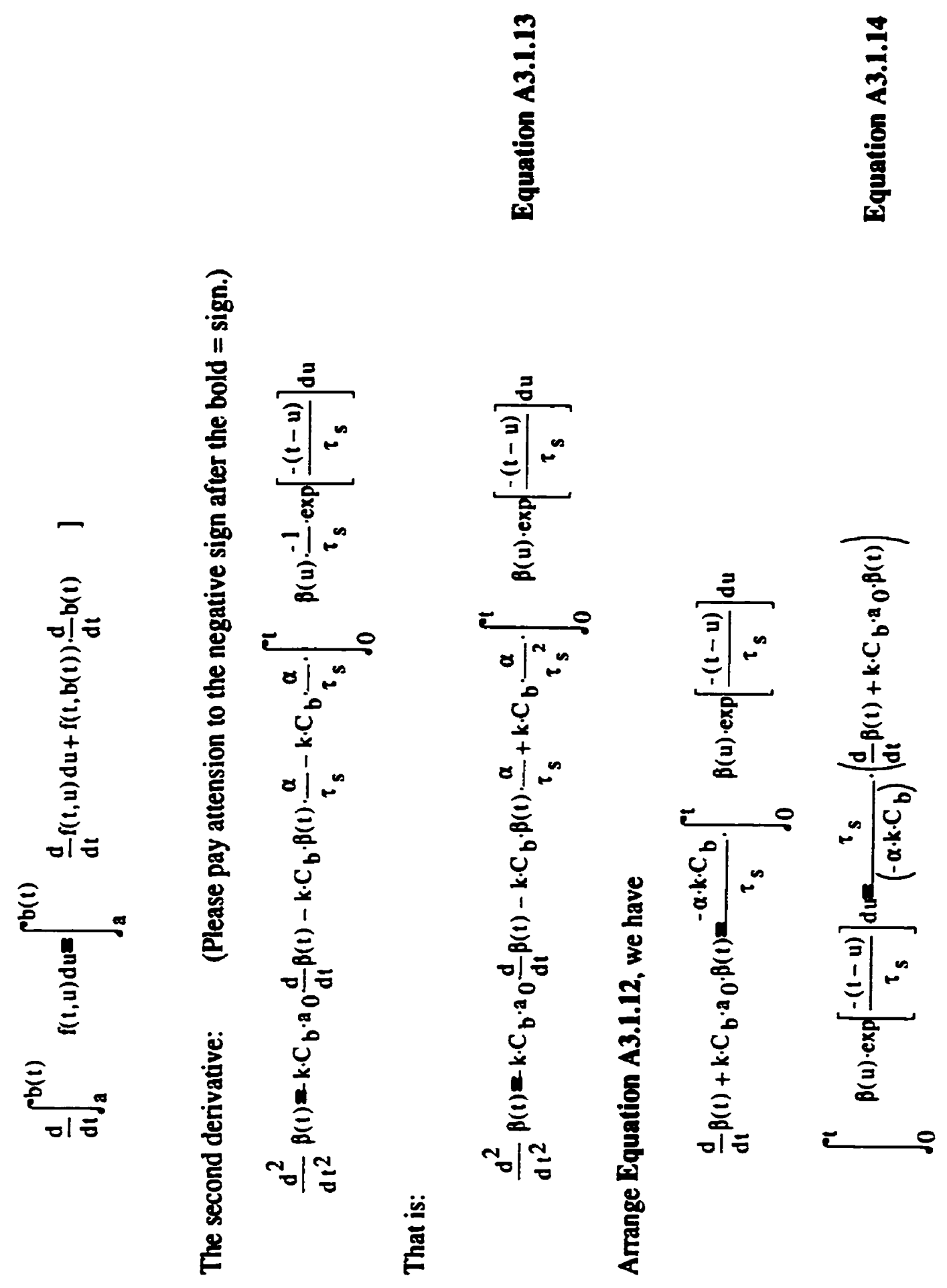


Combine Equation A3.1.14 and Equation A3.1.13, we have:

$$
\frac{d^{2}}{d t^{2}} \beta(t)=k \cdot C_{b} \cdot a 0 \frac{d}{d t} \beta(t)-k \cdot C_{b} \cdot \beta(t) \cdot \frac{\alpha}{\tau_{s}}+k \cdot C_{b} \cdot \frac{\alpha}{\tau_{s}^{2}} \cdot\left[\frac{\tau_{s}}{\left(-\alpha \cdot k \cdot C_{b}\right)} \cdot\left(\frac{d}{d t} \beta(t)+k \cdot C_{b} \cdot a 0 \cdot \beta(t)\right)\right]
$$

Rearrange the above equation, we have:

$$
\frac{d^{2}}{d t^{2}} \beta(t)+\left(k \cdot c_{b} \cdot a_{0}+\frac{1}{\tau_{s}}\right) \frac{d}{d t} \beta(t)+\frac{k \cdot C_{b} \cdot\left(\alpha+a_{0}\right)}{\tau_{s}} \cdot \beta(t)=0 \quad \text { Eq [9a] } \quad \text { Equation A3.1.15 }
$$

Equation A3.1.15 is a linear, homogeneous second-order differential equation. As mentioned in Eijk \& Stuar' paper [1], this equation is the analog of the differential equation describing the movement of a damped pendulum. It's solution can be found in a mathematical or physical handbook [3].

In solving Eq [9a] Equation A3.1.15, the following boundary conditions are used:

$$
\beta(0)=1 \quad\left(\frac{d}{d t} \beta(t)\right)_{\infty}=k \cdot C_{b} \cdot a_{0}
$$

And if the discriminant $D$ is defined as

$$
D\left(k, C_{b}, \tau_{s}, a_{0}, a\right):=\left[\left(1+k \cdot C_{b} \cdot \tau_{s} \cdot a_{0}\right)^{2}-4 \cdot k \cdot C_{b} \cdot \tau_{s} \cdot a, 0 \cdot\left(1+\frac{\alpha}{a_{0}}\right)\right]
$$




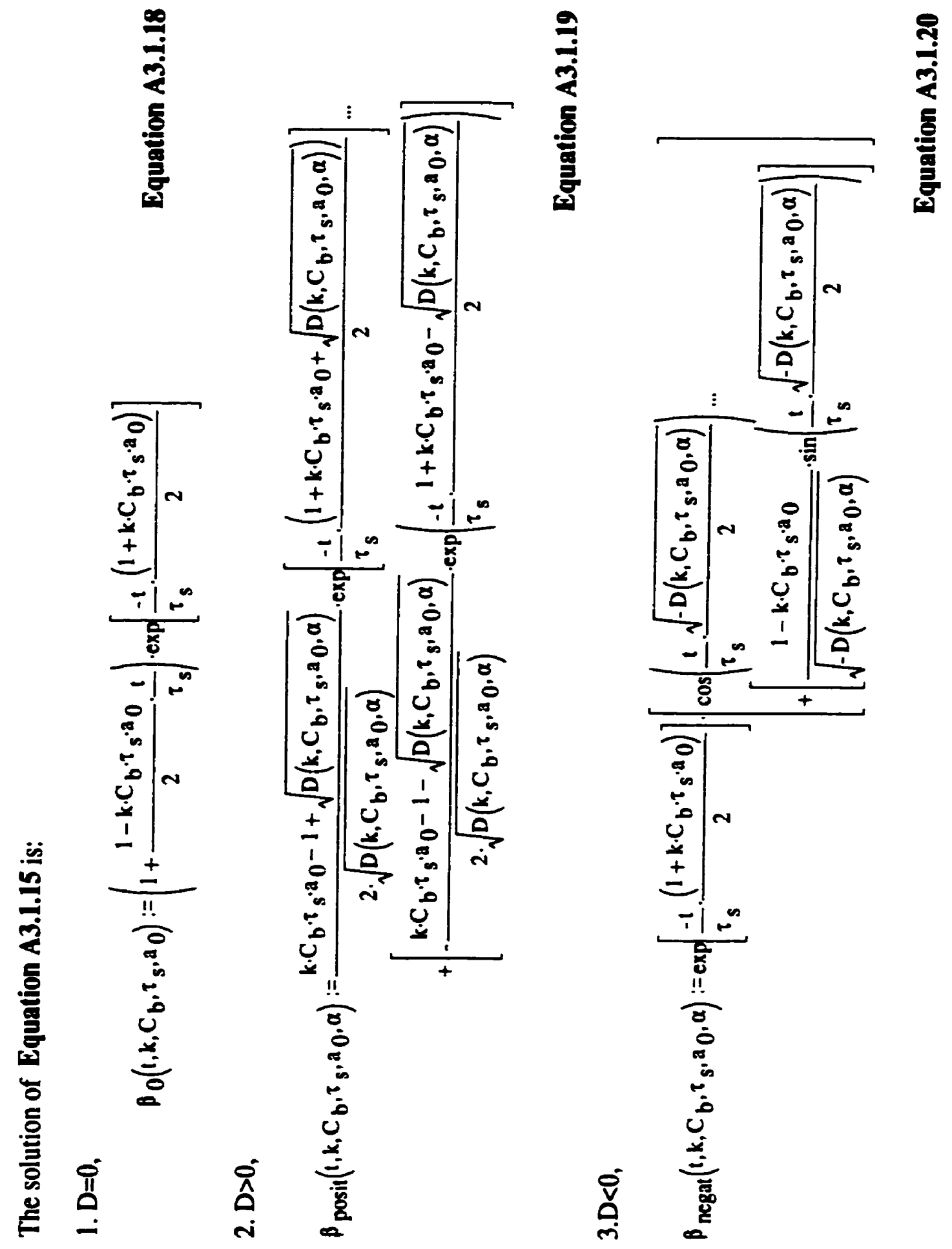


q

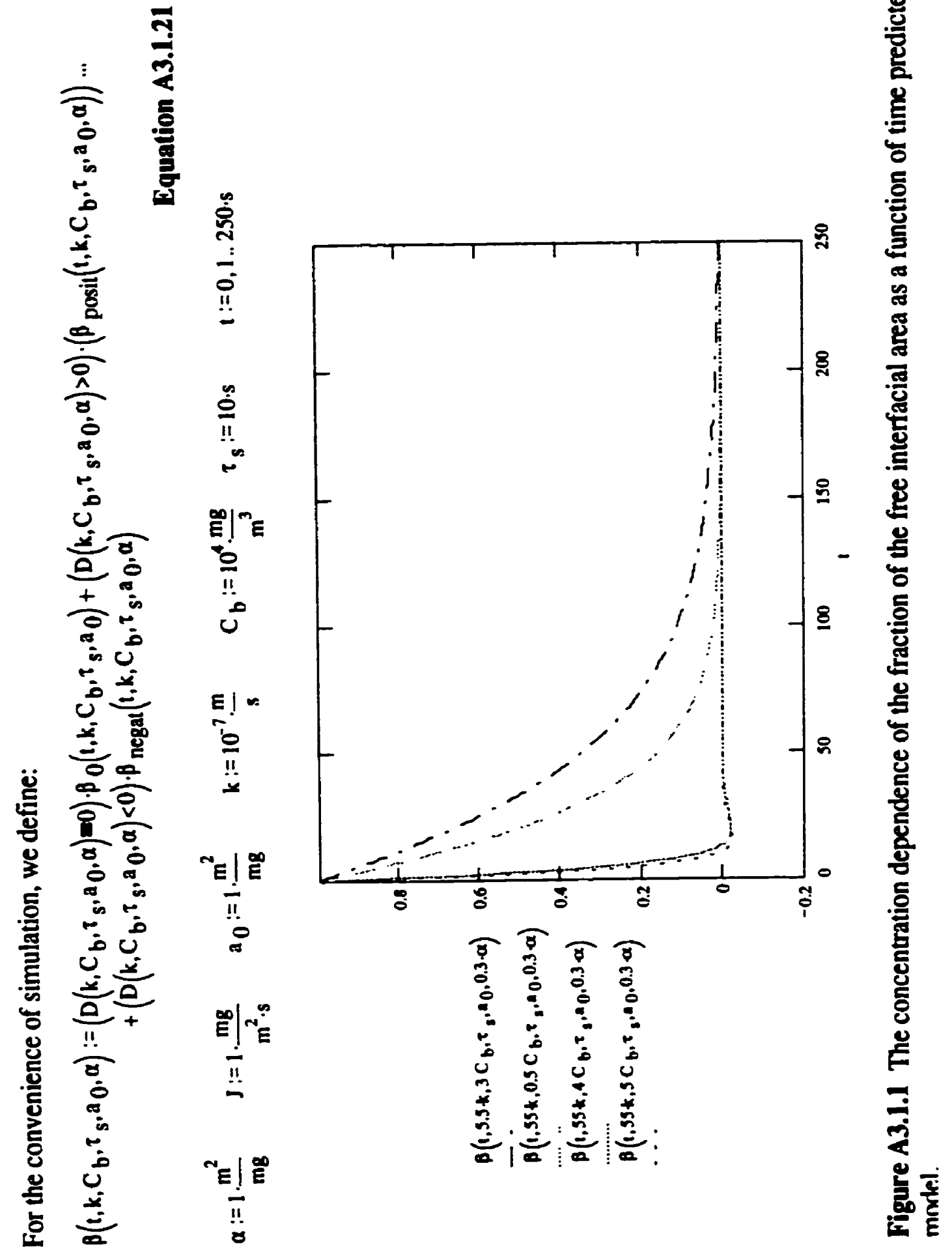


Figure A3.1.1 indicates that $\beta(t)$ is not a monotonically decreasing function of $t$ and it can be negative.

By integrating the both sides of Equation A3.1.10, the interfacial concentration of the adsorbed polymer is:

$$
\Gamma\left(t, k, C_{b}, \tau_{s}, a_{0}, \alpha\right):=k \cdot C_{b} \cdot \int_{0}^{t} \beta\left(u, k, C_{b}, \tau_{s}, a_{0}, \alpha\right) d u \quad \text { Equation A3.1.22 } \quad t:=0,1 \ldots 300 . s
$$

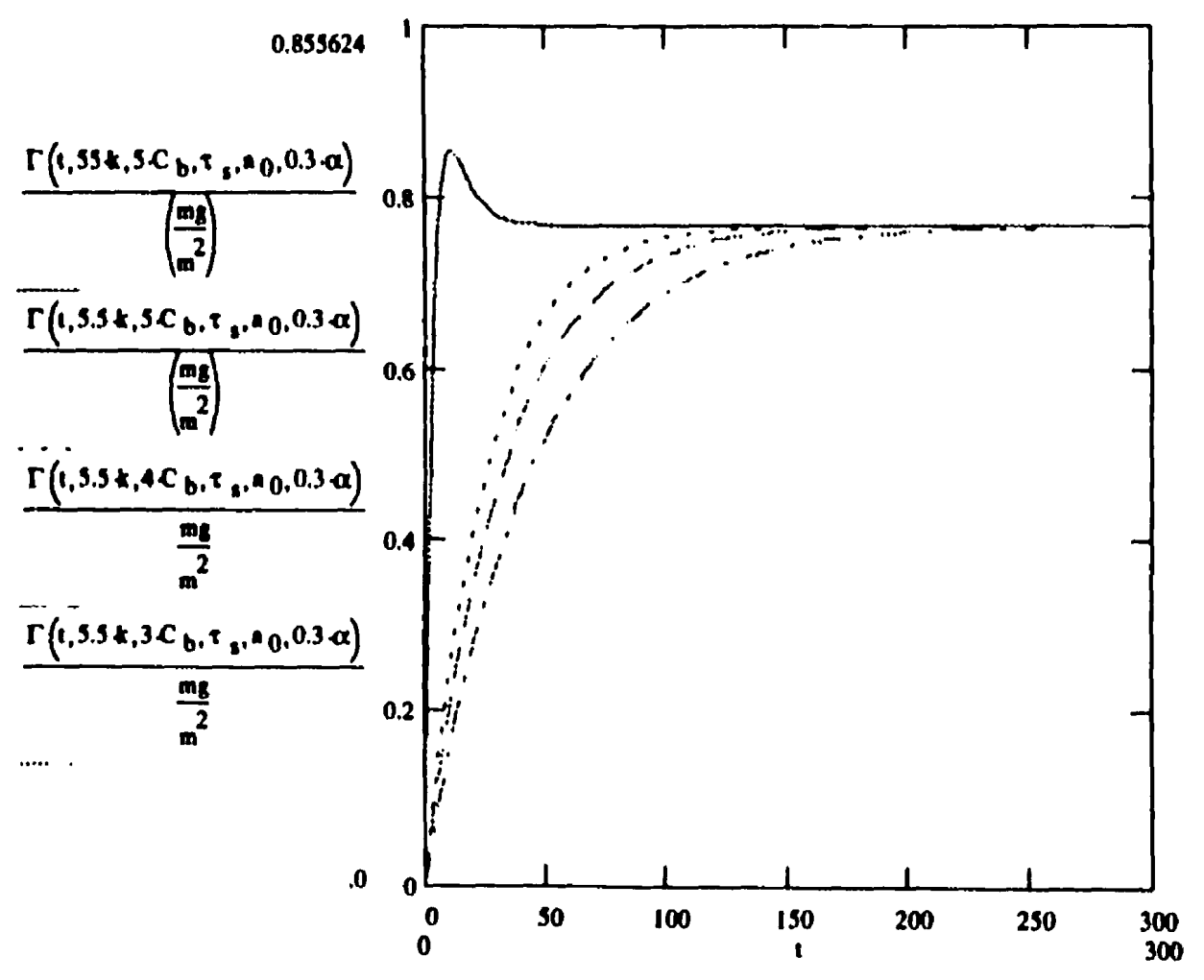

Figure A3.1.2 The bulk concentration dependence of $\Gamma(t)$, 
Figure A3.1.2 indicates that, $\Gamma(t)$ is also not a monotonically increasing function of $t$, after it reaches its maximum, it starts to decrease slightly, and then converges to a constant value.

Because of the mathematical behaviours of both $\beta(t)$ and $\Gamma(t)$, we choose the interfacial concentration at the first time when $\beta(t)$ reaches zero as the maximum interfacial concentration of adsorbed polymer. That is,

$$
\begin{aligned}
& t:=0 . s \\
& t_{\max }\left(k, C_{b}, \tau_{s}, a_{0}, \alpha\right):=\operatorname{rog}\left(\beta\left(t, k, C_{b}, \tau_{s}, a_{0}, \alpha\right), t\right)
\end{aligned}
$$

For example,

$$
\begin{aligned}
& t_{\max }\left(5.5 \cdot k_{,} 5 \cdot \mathrm{C}_{\mathrm{b}}, \tau_{\mathrm{s}}, \mathrm{a}_{0}, 0.3 \alpha\right)=185.534 \cdot \mathrm{s} \\
& t_{\max }\left(5.5 \cdot k, 4 \cdot \mathrm{C}_{\mathrm{b}}, \tau_{\mathrm{s}}, \mathrm{a} 0,0.3 \cdot \alpha\right)=239.480 \cdot \mathrm{s} \\
& t_{\max }\left(5.5 \cdot k, 3 \cdot C_{b}, r_{s}, a_{0}, 0.3 \cdot \alpha\right)=327.404 \cdot s \\
& \Gamma_{\max }\left(l, k_{,} C_{b}, \tau_{s}, a_{0}, \alpha\right):=\Gamma\left(r_{\max }\left(k_{1}, C_{b}, \tau_{s}, a_{0}, \alpha\right), k_{,} c_{b}, z_{s}, a_{0}, a\right)
\end{aligned}
$$

And define the interfacial concentration of adsorbed polymer as:

$$
\begin{aligned}
r\left(t, k, C_{b}, \tau_{s}, a_{0}, \alpha\right):= & \Gamma\left(t, k, C_{b}, \tau_{s}, a_{0}, \alpha\right) \cdot\left(t s_{\max }\left(k_{,} C_{b}, \tau_{s}, a_{0}, \alpha\right)\right) \ldots \\
& +\Gamma_{\max }\left(t, k_{,}, C_{b}, \tau_{s}, a_{0}, \alpha\right) \cdot\left(t>t_{\max }\left(k_{,}, C_{b}, \tau_{s}, a_{0}, \alpha\right)\right)
\end{aligned}
$$

To see how close this model is to the real data, we use the following $\gamma$ vs $\Gamma$ relationship based on SCF calculation at $25^{\circ} \mathrm{C}$ (see Appendix 3.2) to link the $\Gamma$ predicted by this model to $\gamma$ : 


$$
\gamma_{25}(\Gamma):=\left[40.8085+\frac{72.14-40.8085}{1+\left[\frac{\Gamma}{0.527 \cdot \frac{\mathrm{mg}}{\mathrm{m}^{2}}}\right]^{5.9932}}\right] \cdot \frac{\mathrm{mJ}}{\mathrm{m}^{2}}
$$

\section{Equation A3.1.26}

According to the fitting result of Hua \& Rosen's equation, we have the experimental $\gamma$ as a function of time for the dynamic surface tension of $30 \mathrm{ppm} \mathrm{L-6B}$ water solution as:

$$
\gamma_{\text {L6B3025 }}(t):=\left[43.10+\frac{72.14-43.10}{1+\left(\frac{t}{56.8 \cdot 5}\right)^{2.4113}}\right] \cdot \frac{\mathrm{mJ}}{\mathrm{m}^{2}} \quad t:=0,2 . .500 \cdot \mathrm{s}
$$

\section{Equation A3.1.27}

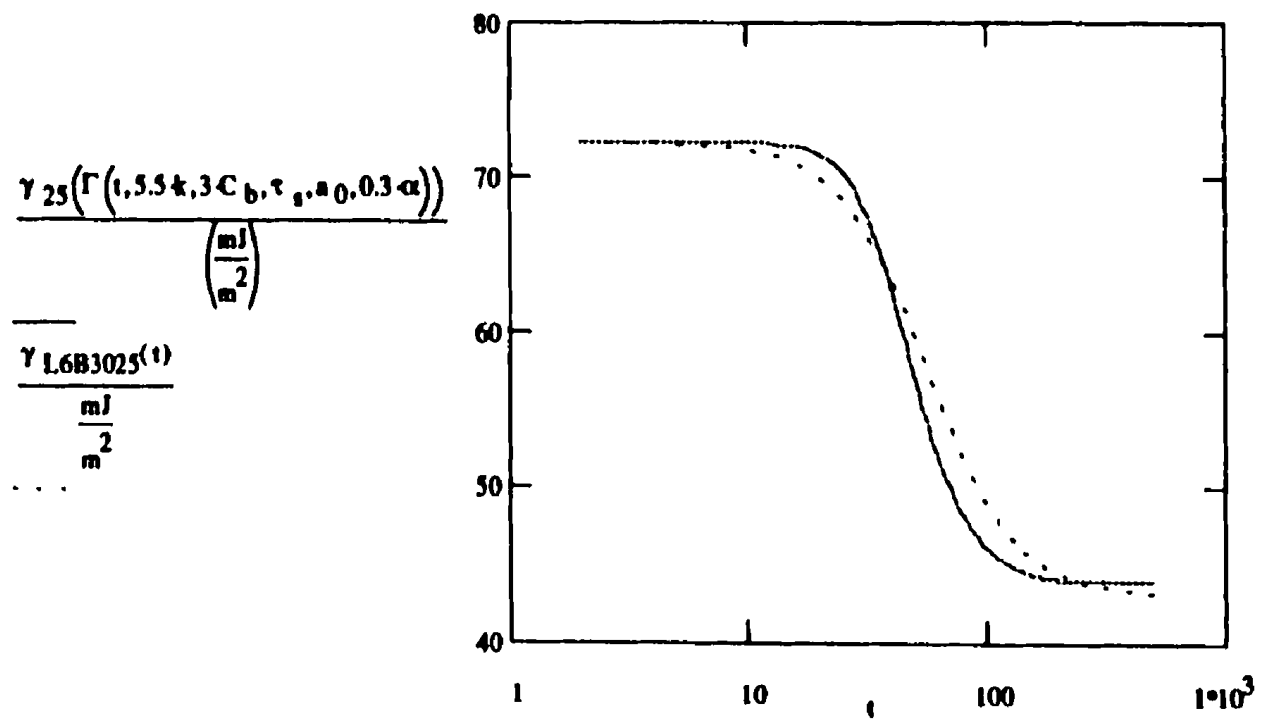

Figure A3.1.3 An example of the dynamic surface tension predicted by this model. 


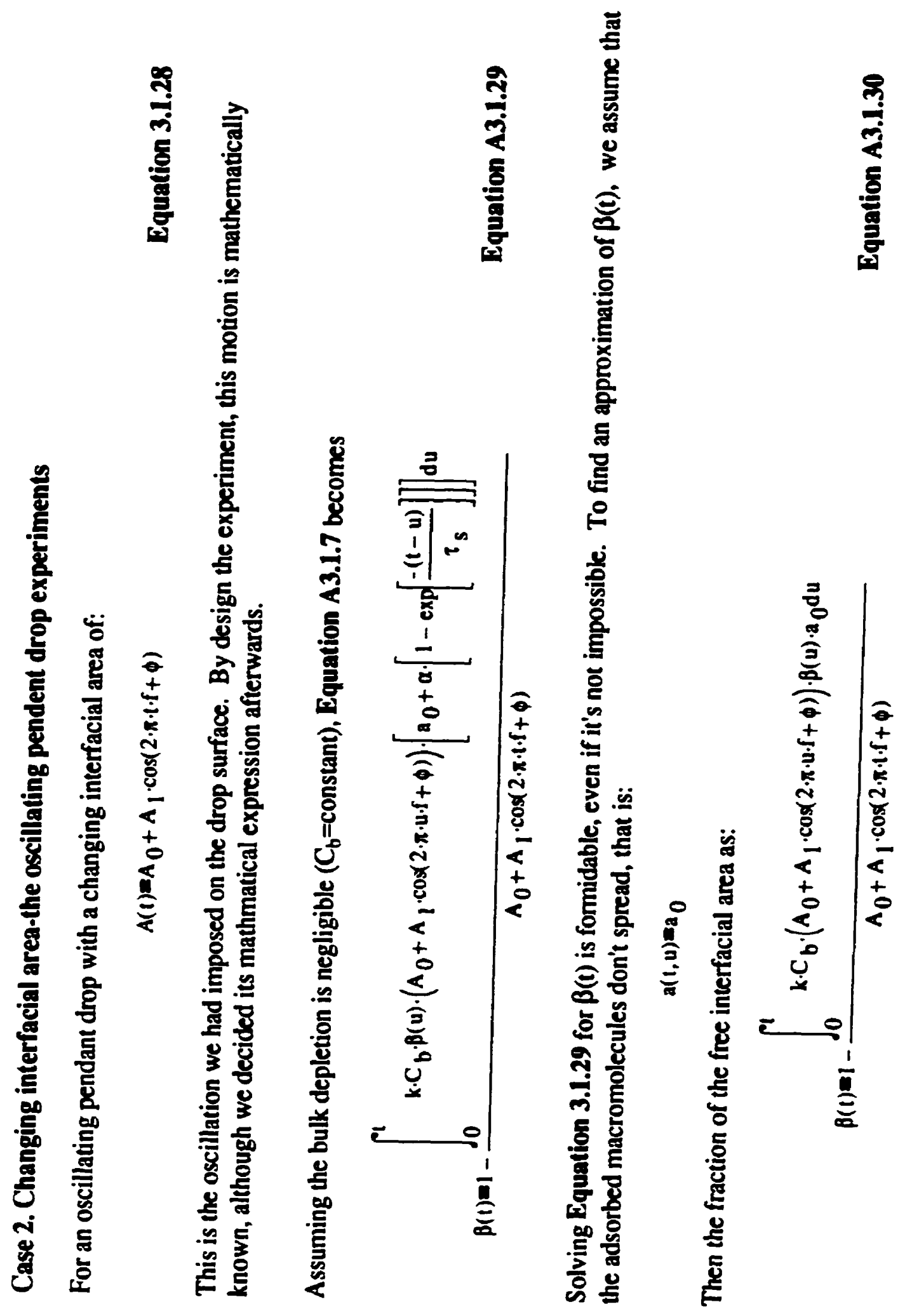


昌

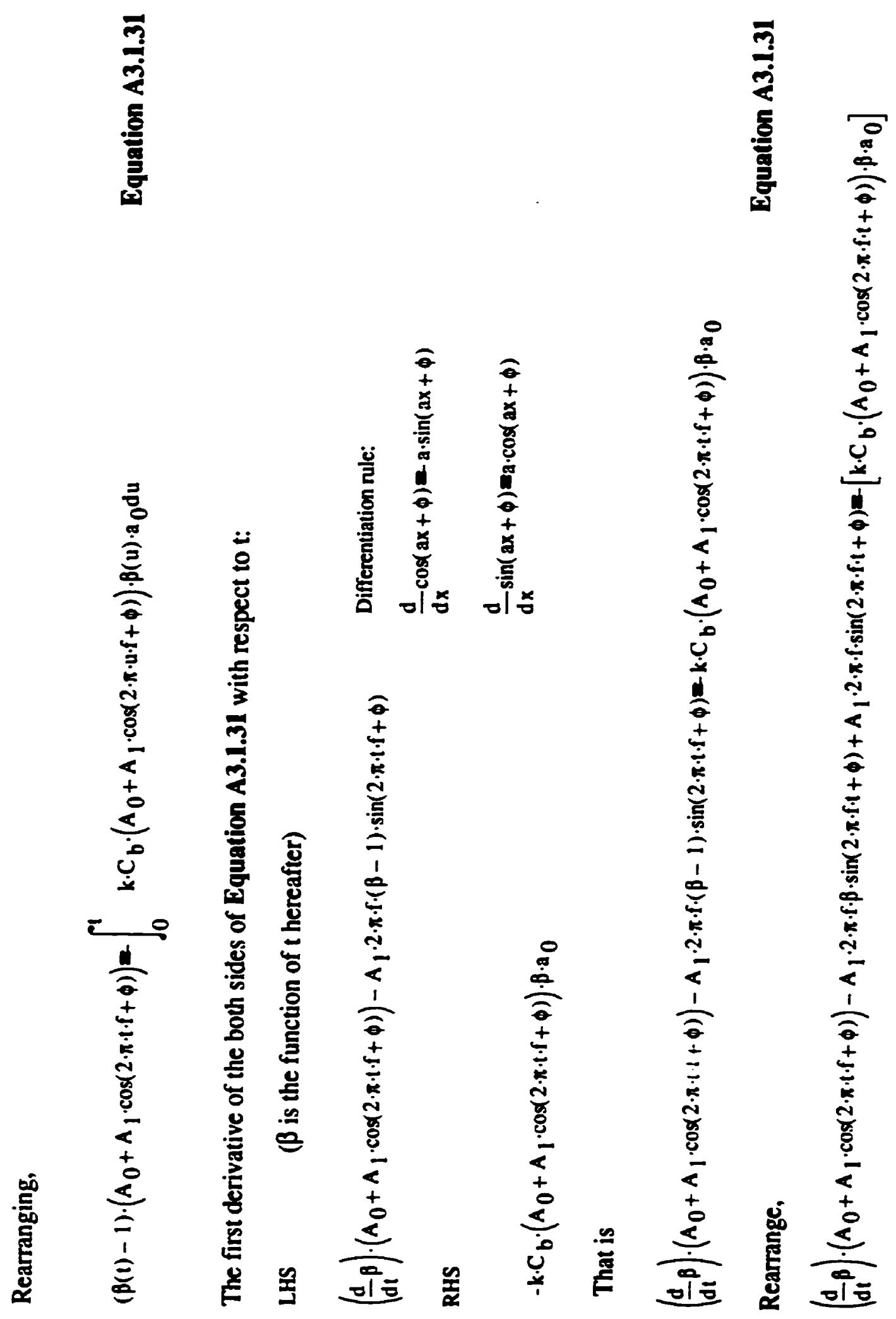


里

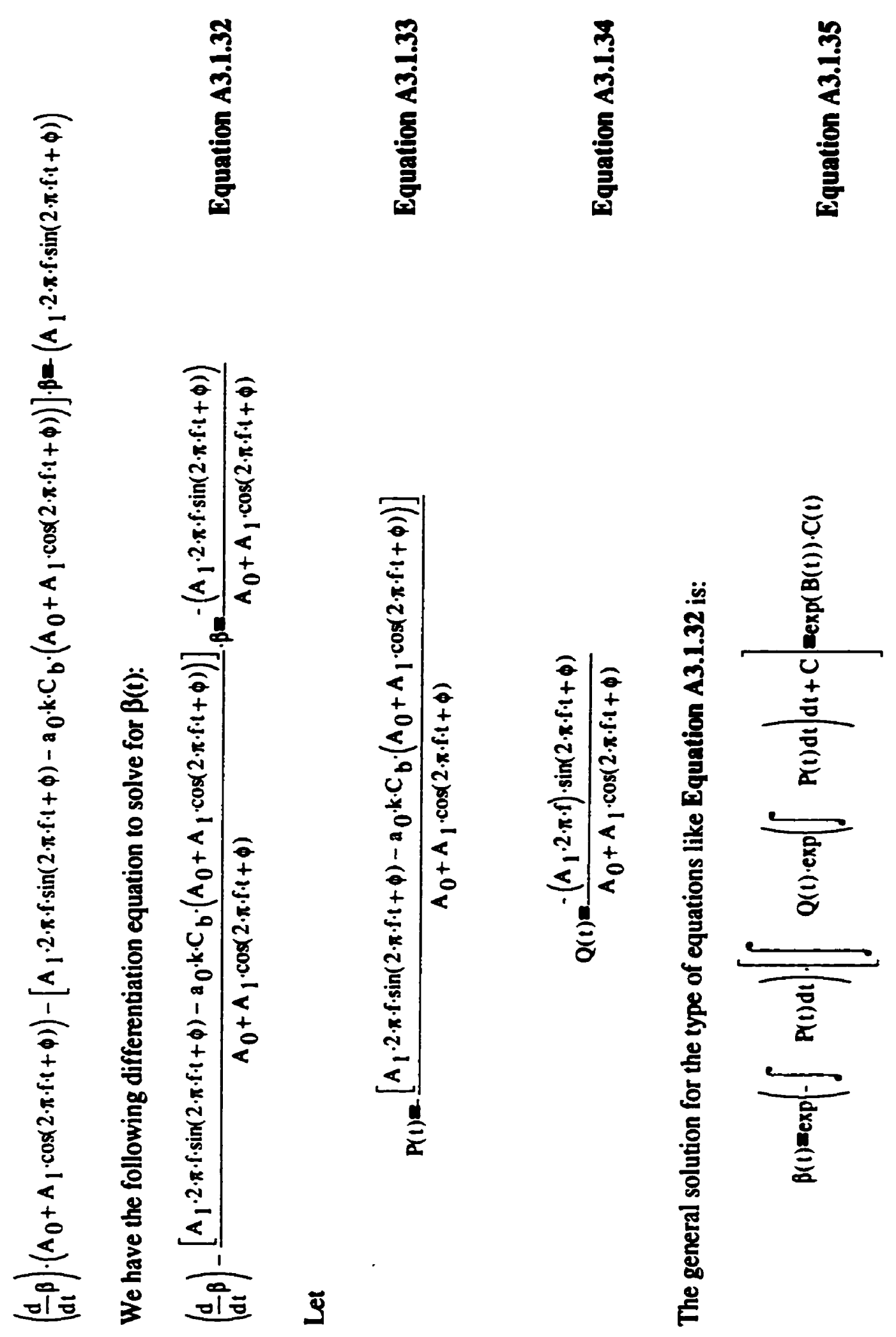


我

产

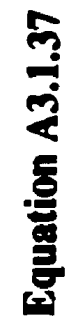
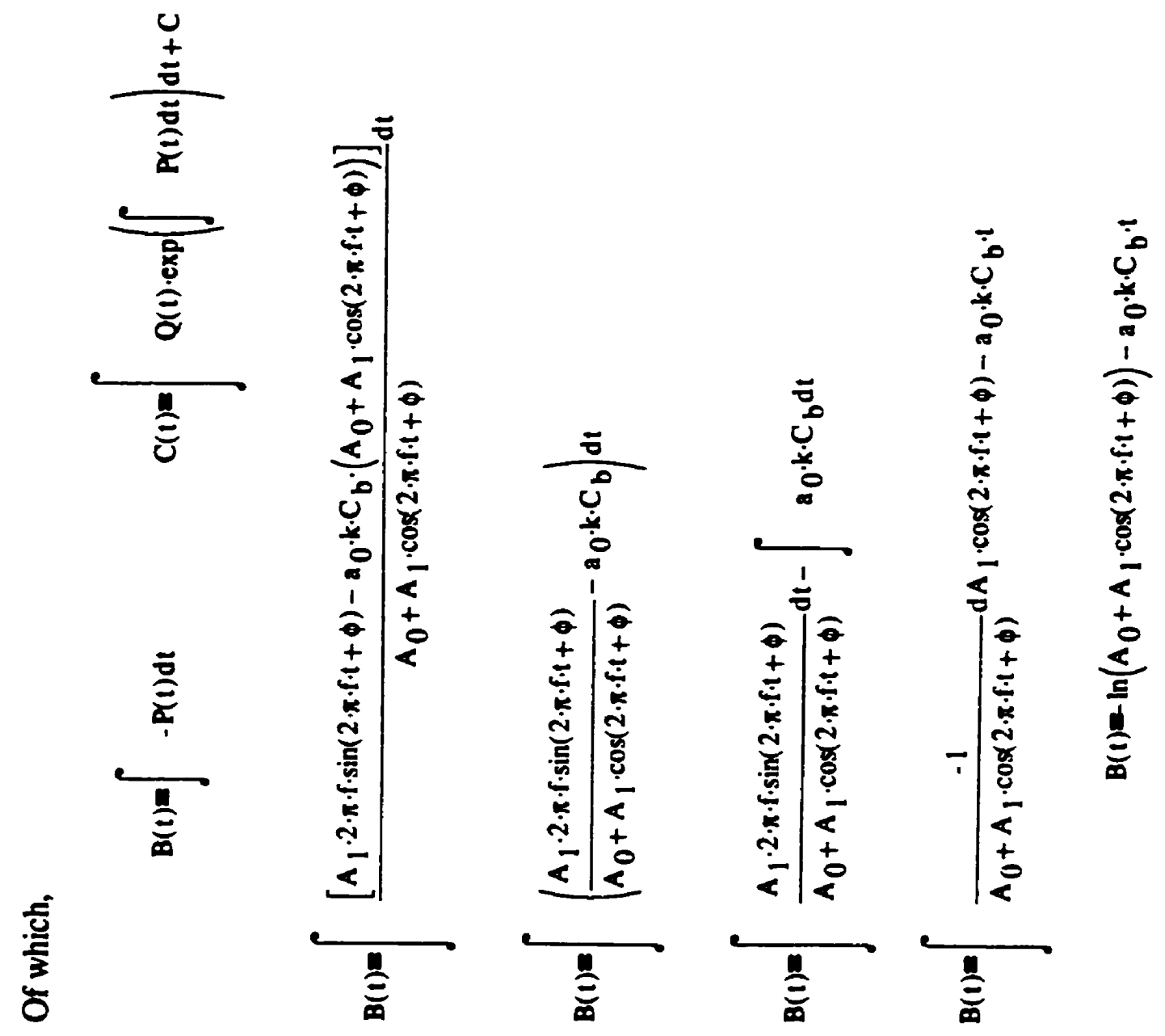

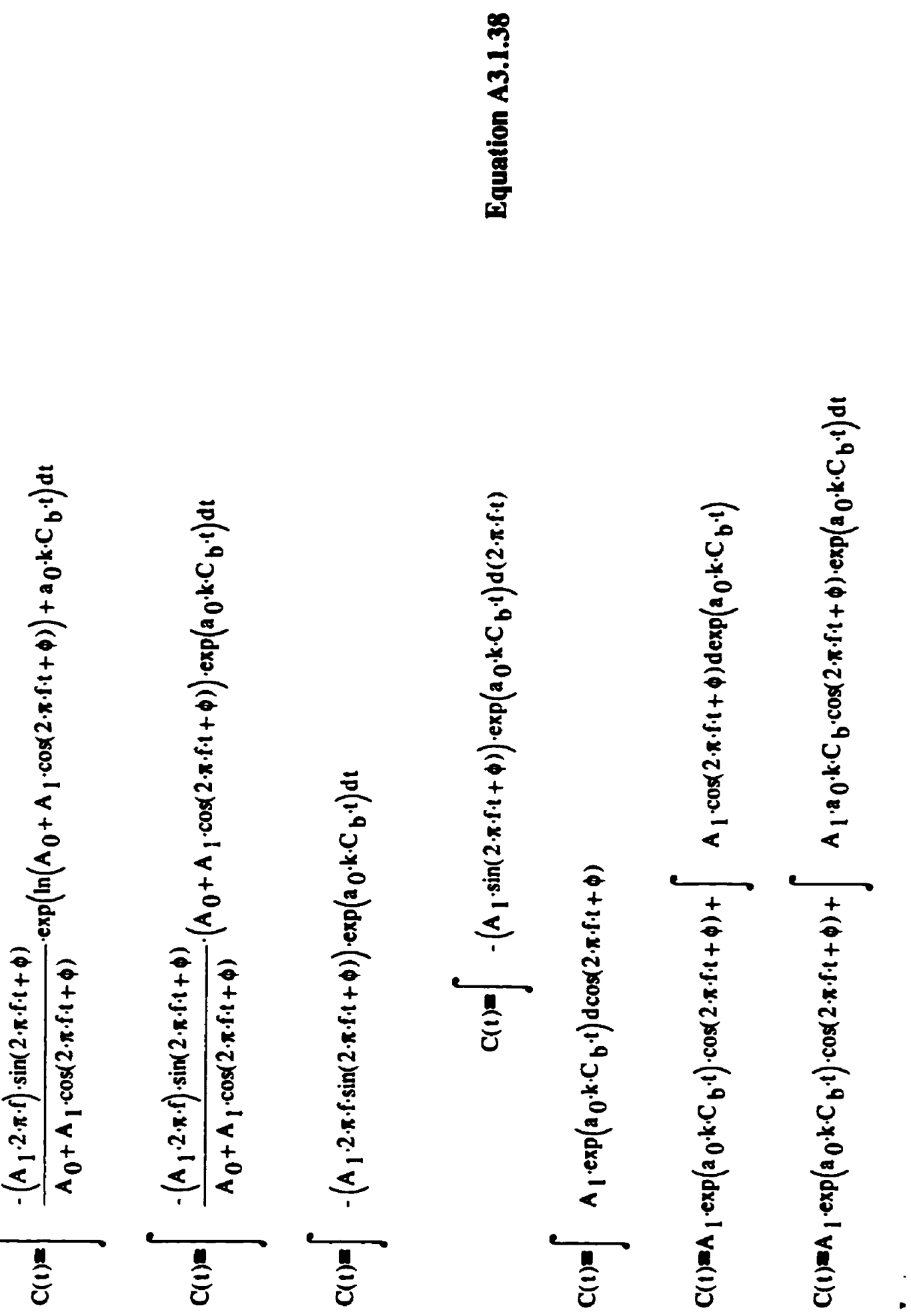
?

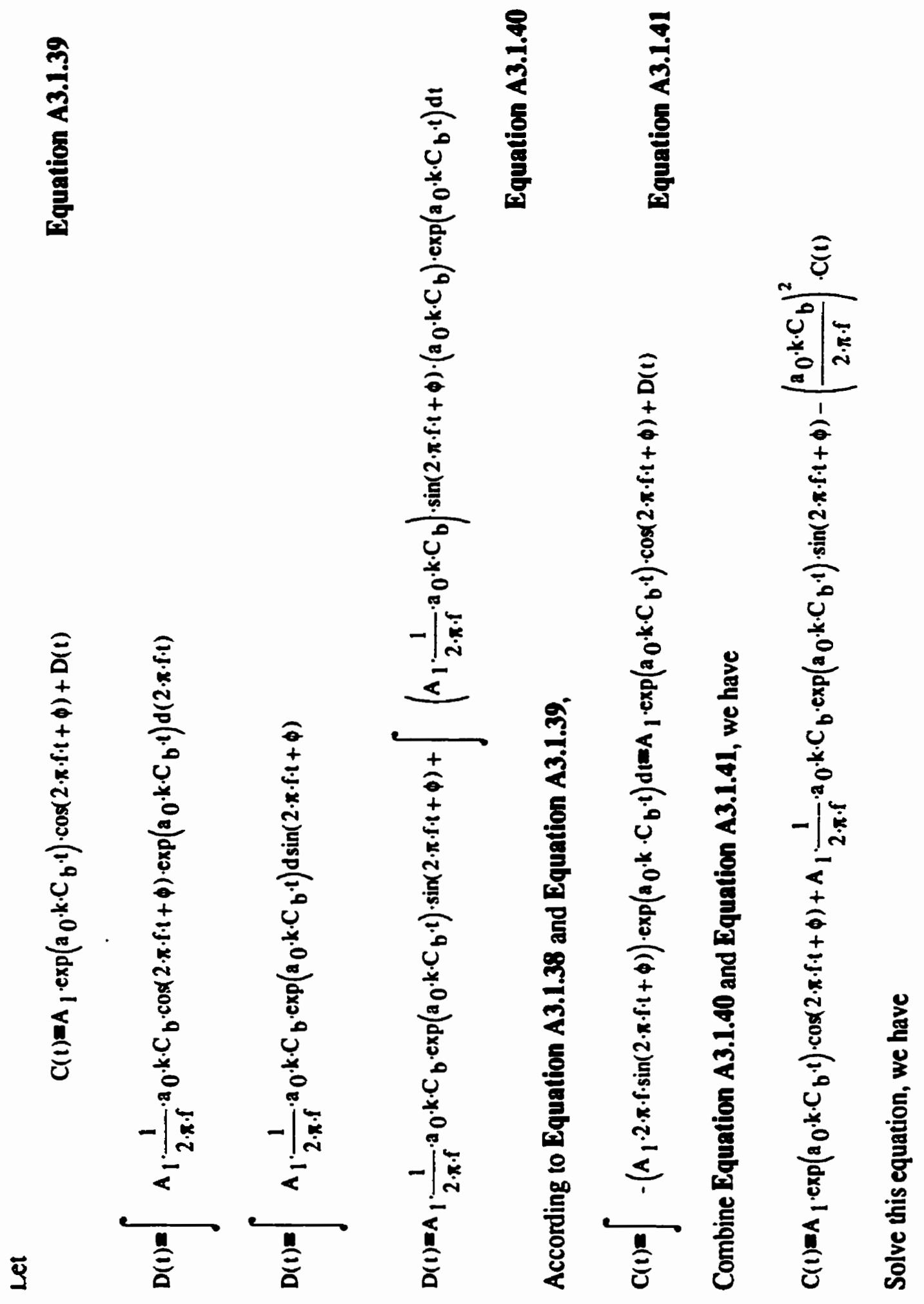


요
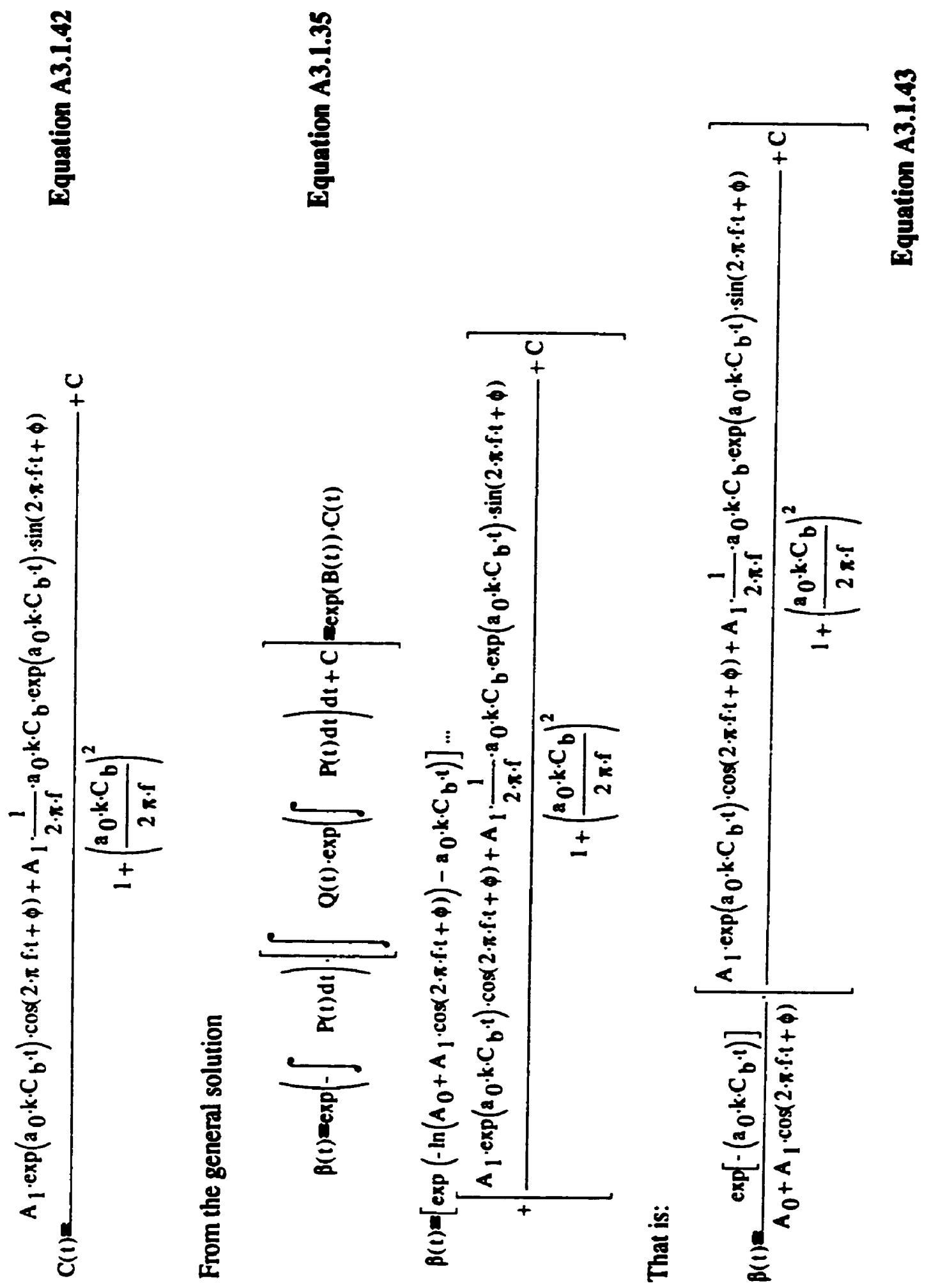
홀

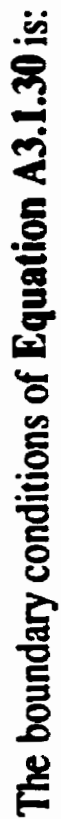
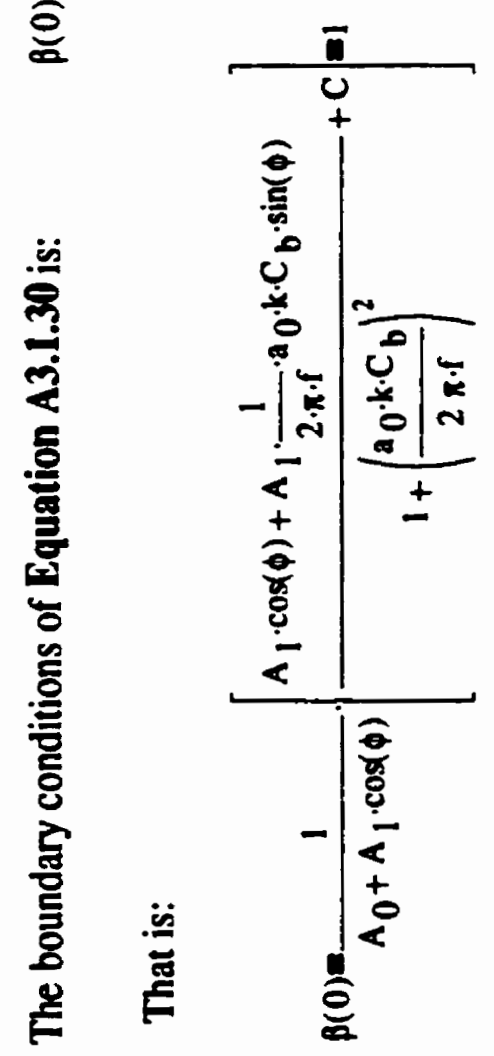
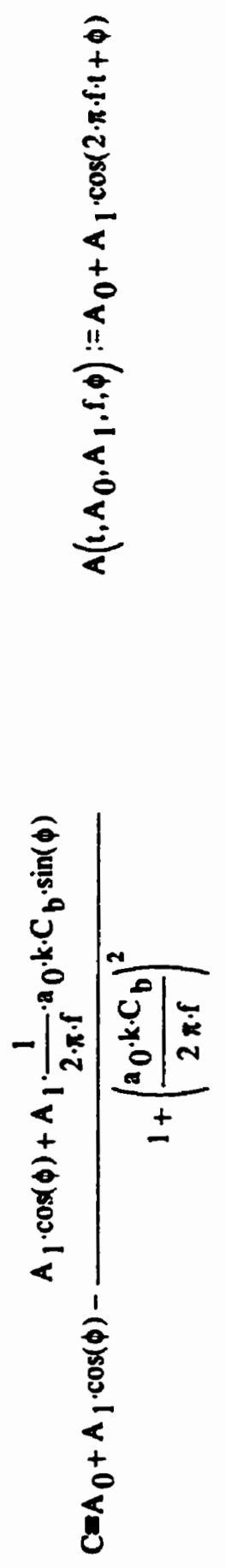

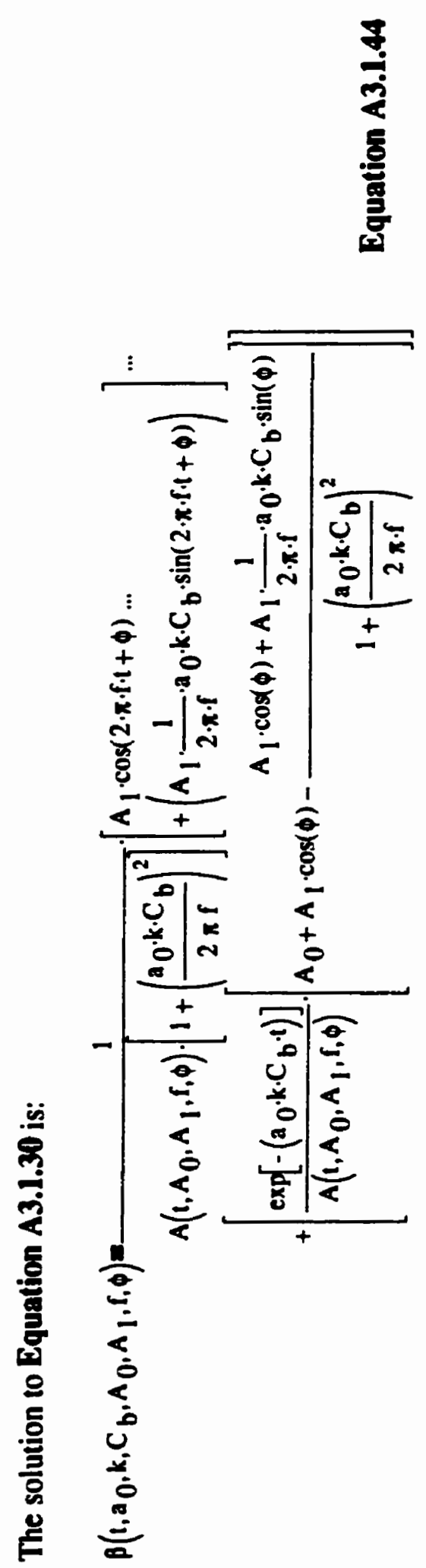


몸

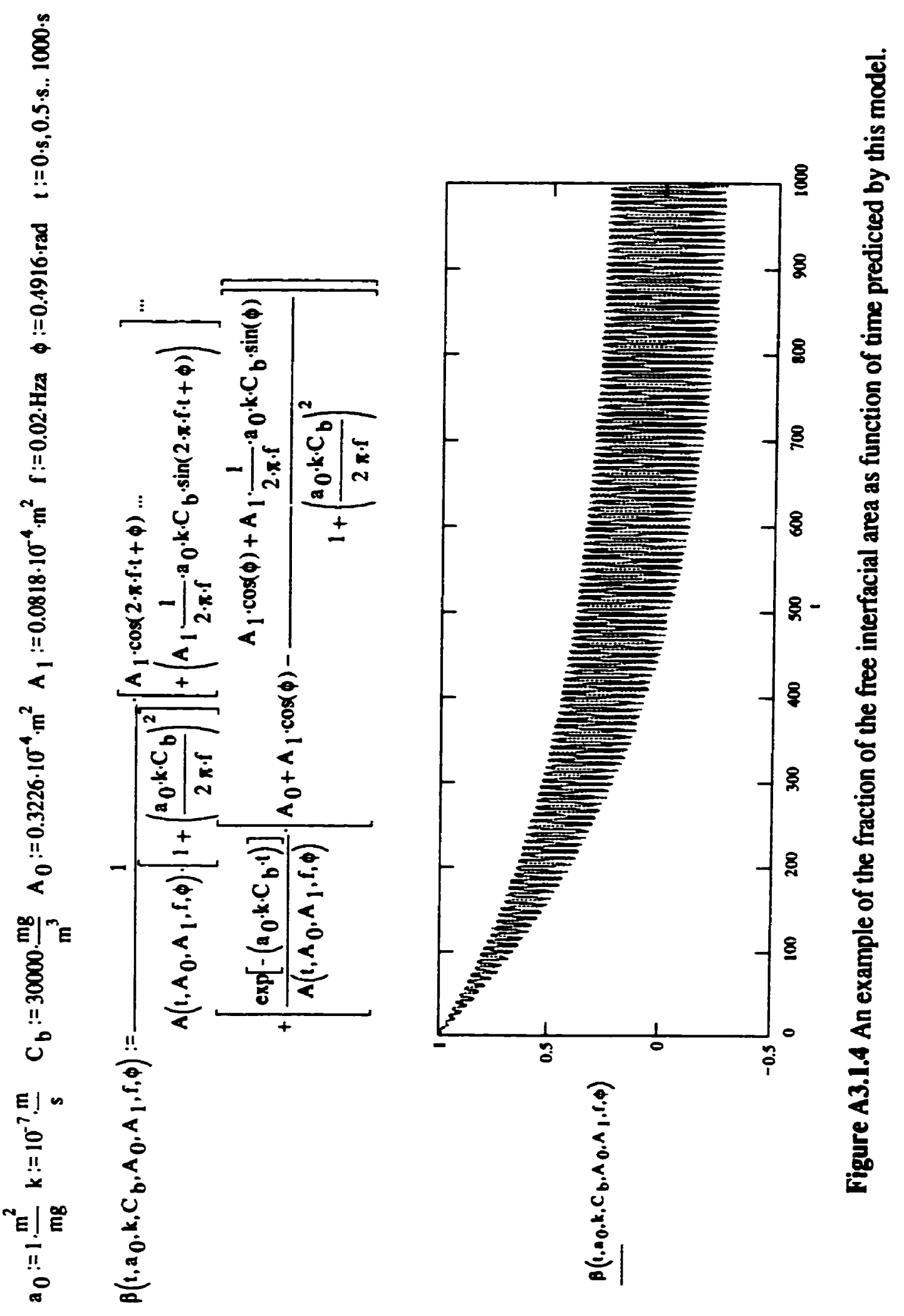



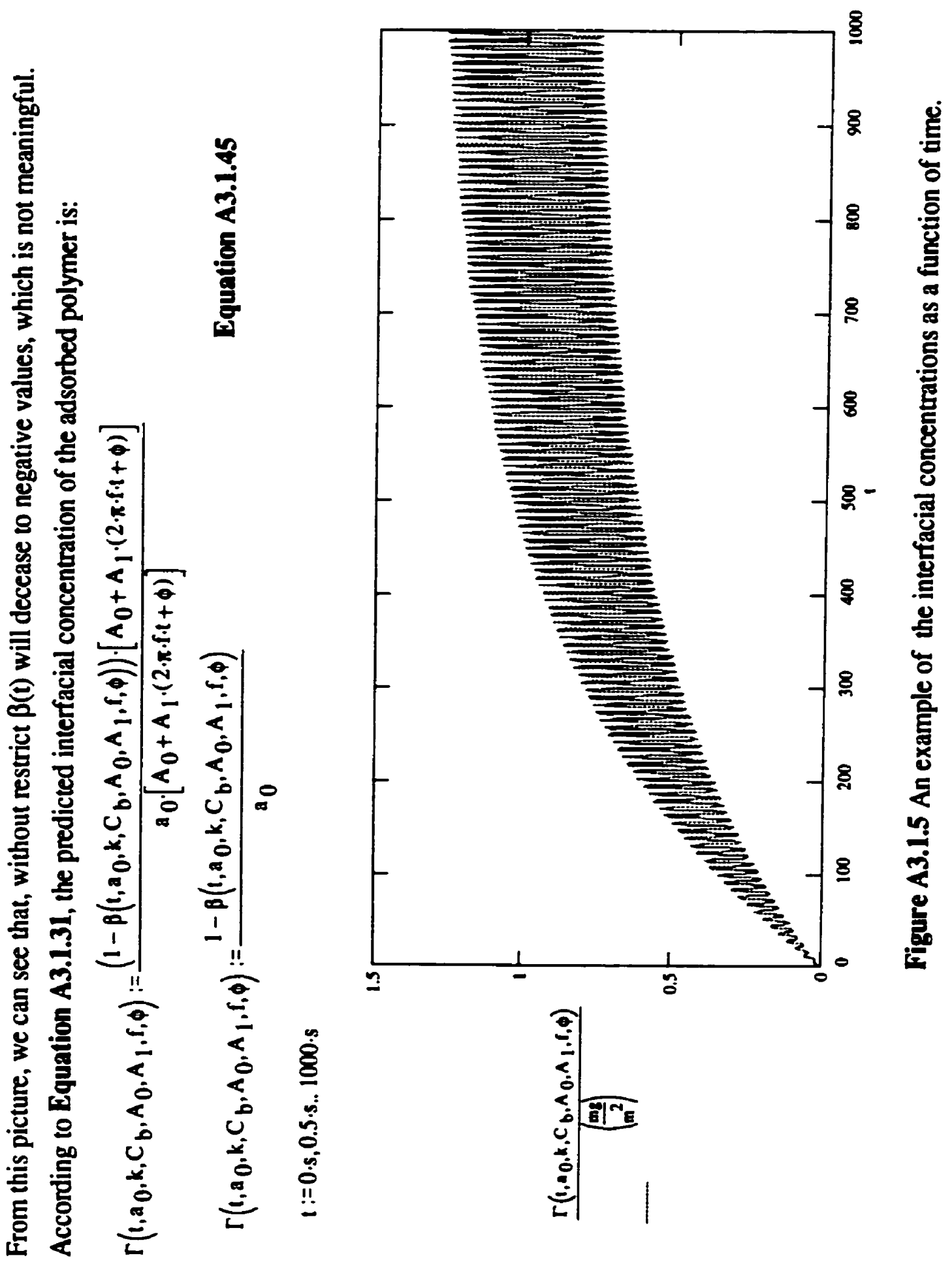
苞

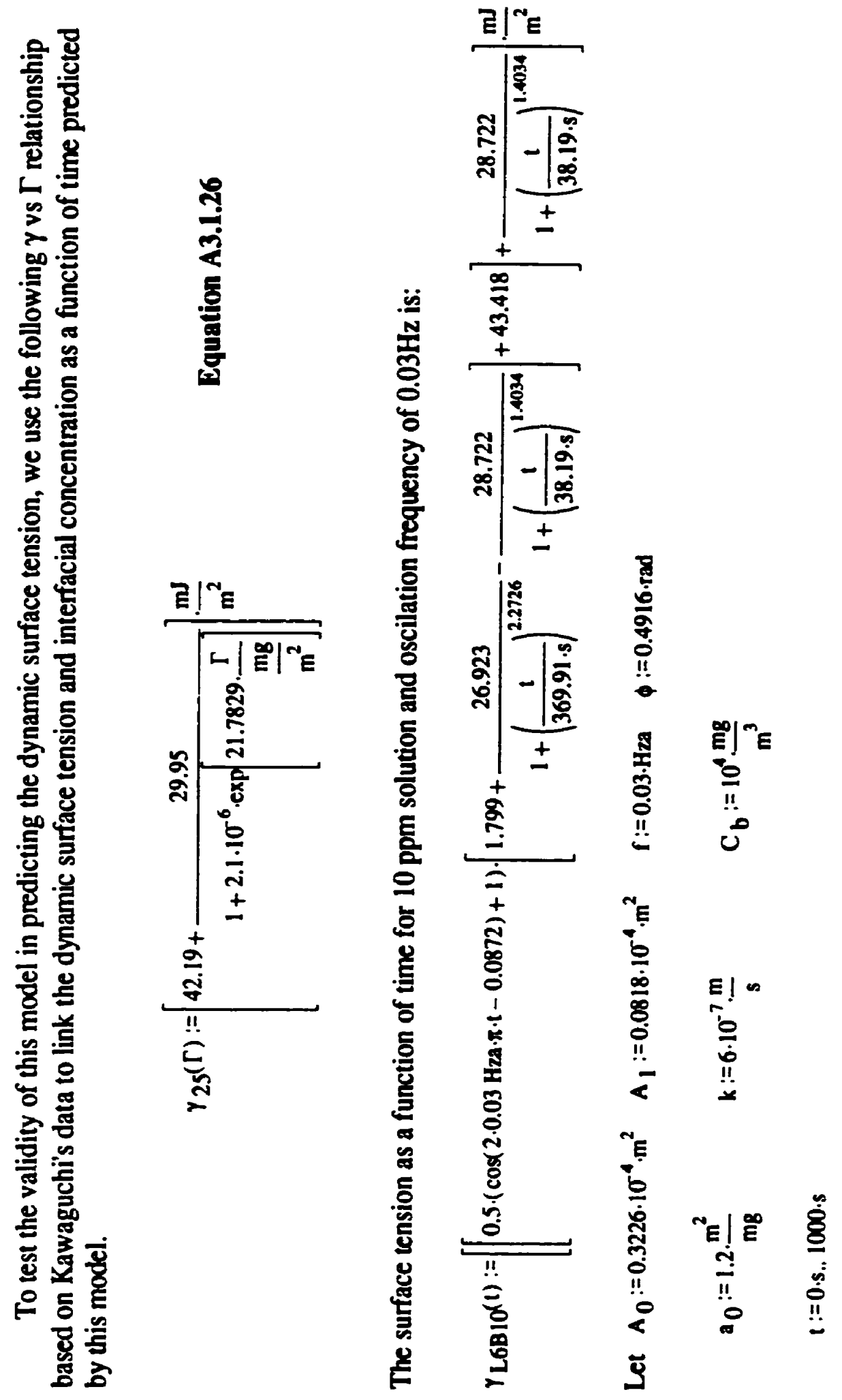




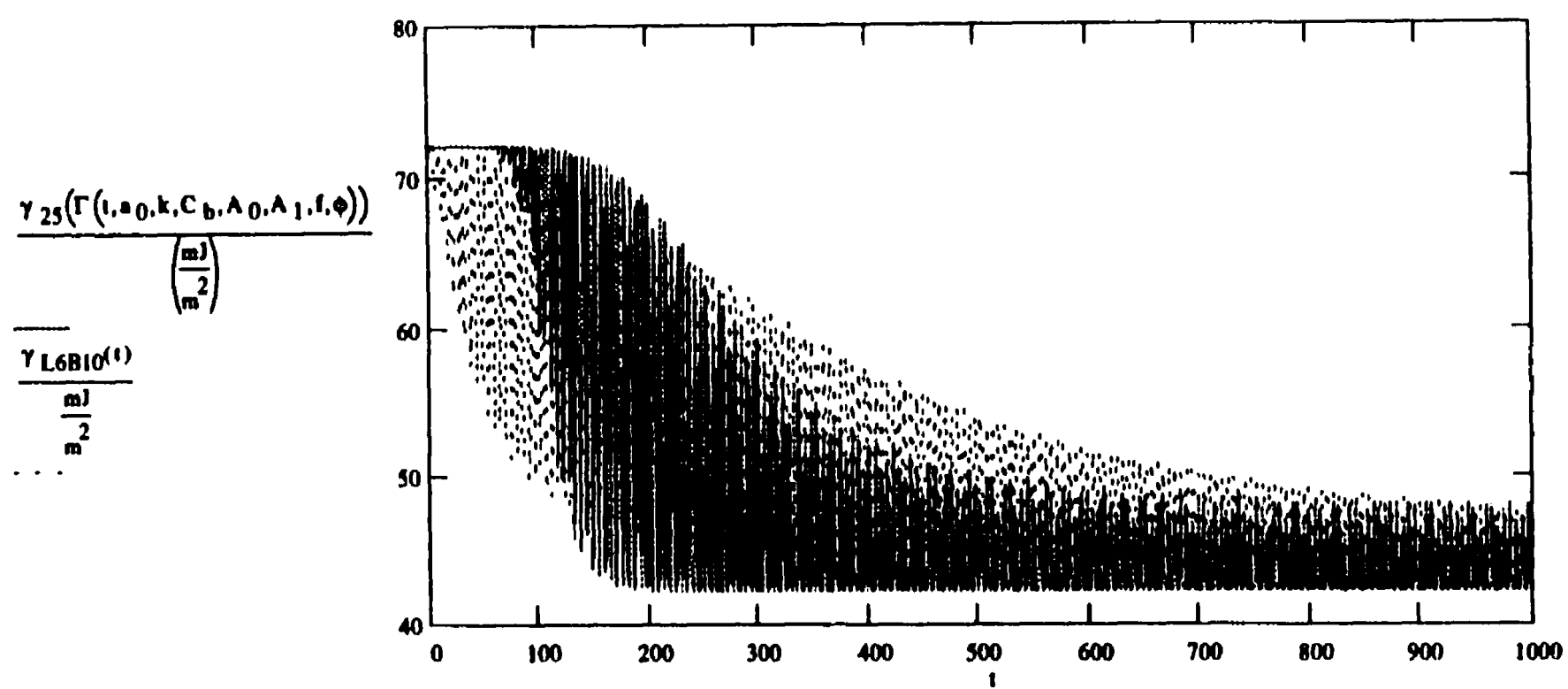

Figure A3.1.6 An example of the model prediction of the dynamic surface tension as a function of time and the experimental $\gamma$ vs $\Gamma$ relationship.

\section{References:}

1. van Eijk, M. C. P.; Cohen Stuart, M. A., "Polymer Adsorption Kinetics: Effects of Supply Rate", Langmuir, 13, 5447(1997).

2. Pefferkom, E.and Elaissari, A., "Adsorption-Desorption Processes in Charged Polymer/Colloid Systems: Structural Relaxation of Adsorbed Macromolecules", J. Colloid Interface Sci., 138, 187 (1990).

3. West, R. C., Astle, M. J., Eds. CRC Handbook of Chemistry and Physics, 62nd ed.; CRC Press: Boca Raton, FL, Page A87., 1981 


\section{Appendix 3.2 Fitting the SCF Calculation Data}

The purpose of this worksheet is to fit the SCF calculation result to the following equation:

$$
\gamma(\Gamma)=A+\frac{72.14-A}{1+\left(\frac{\Gamma}{B}\right)^{E}}
$$

Calculated

$\Gamma_{1} \quad x_{1}$

$\mathrm{m} / \mathrm{m}^{2} \mathrm{~mJ} / \mathrm{m}^{2}$

o $72.14 \mathrm{E}=$

$0.0034171 .9966 \mathrm{~B}=$

$0.1624771 .2834 \mathrm{~A}=$

$0.2996 \quad 69.1485$

$0.4555762 .8307 \operatorname{sum}(i)=$

0.49759 .3655

$0.5116157 .8889 \mathrm{SSE}=\operatorname{sum}\left(\gamma_{1}-\gamma_{\ell}\right)^{2}$

$0.5372355 .6443 \mathrm{SST}=\operatorname{sum}\left(\gamma_{i}^{2}\right)-\left(\operatorname{sum}\left(\gamma_{j}\right)\right)^{2} / \operatorname{sum}(i)$

$0.608950 .7665 \mathbf{R}^{2}=1-S S E / S S T$

$0.70862 \quad 45.395$

0.7838241 .9271 SSE

$1.5 \quad 41.9271 \operatorname{sum}\left(x^{2}\right)$

$\operatorname{sum}(\boldsymbol{\gamma})$

SST

$R^{2}=1 . S S E / S S T \quad 0.99414$
Fitted

$\gamma_{i s} \quad\left(\boldsymbol{\gamma}-\boldsymbol{\gamma}_{i, s}\right)^{2} \boldsymbol{\gamma}^{2}$

$\mathrm{mJ} / \mathrm{m}^{2} \quad\left(\mathrm{~mJ} / \mathrm{m}^{2}\right)^{2}\left(\mathrm{~mJ} / \mathrm{m}^{2}\right)^{2}$

$\begin{array}{llll}5.90322 & 72.14 & 0 & 5204.18\end{array}$

$\begin{array}{llll}0.52703 \mathrm{me} / \mathrm{m}^{2} & 72.14 & 0.02058 & 5183.5\end{array}$

$\begin{array}{lllll}40.8085 & \mathrm{~mJ} / \mathrm{m}^{2} & 72.1099 & 0.68309 & 5081.32\end{array}$

$\begin{array}{llll}71.0617 & 3.66031 & 4781.52\end{array}$

$62.8252 \quad 3 E-05 \quad 3947.7$

$\begin{array}{lll}59.1604 & 0.04208 & 3524.26\end{array}$

$\begin{array}{lll}57.8442 & 0.002 & 3351.13\end{array}$

$\begin{array}{lll}55.5889 & 0.00307 & 3096.29\end{array}$

$\begin{array}{lll}50.1743 & 0.35074 & 2577.24\end{array}$

$\begin{array}{lll}45.4563 & 0.00375 & 2060.71\end{array}$

$\begin{array}{llll}43.5535 & 2.64547 & 1757.88\end{array}$

$\begin{array}{lll}40.8736 & 1.10987 & 1757.88\end{array}$

The equation obtained is:

$$
\gamma(\Gamma)=40.8085+\frac{72.14-40.8085}{1+\left(\frac{\Gamma}{0.527}\right)^{5.9032}}
$$




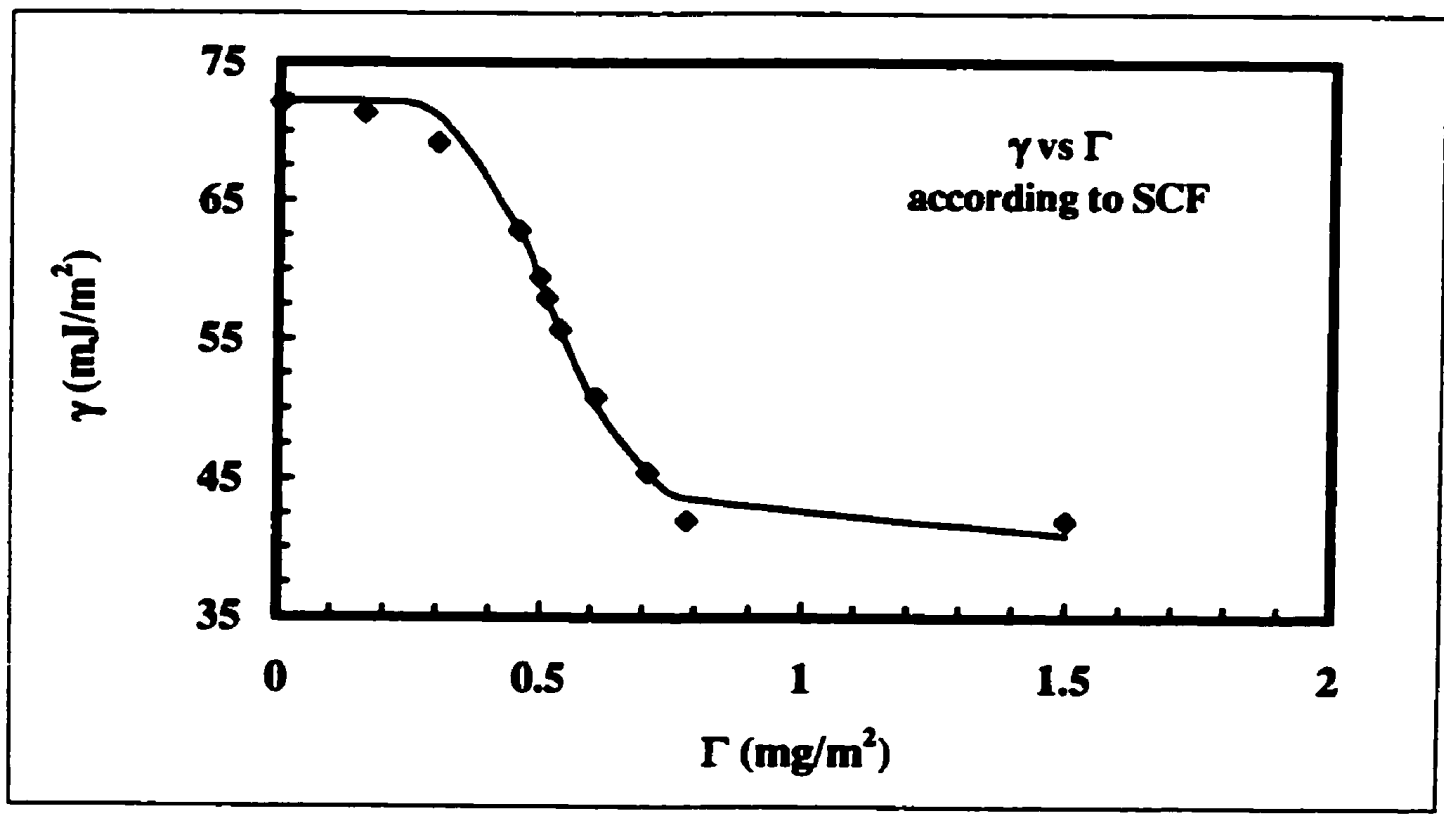




\section{Appendix 3.3 Calculation of the Slopes of the Dynamic Surface Tension Curves of $\mathbf{4 0} \mathbf{~ p p m}$}

The objective of this spreadsheet is to approximate the maximum slopes of the experimental and predicted dynamic surface tension curves of $40 \mathrm{ppm}$ PNIPAM solution at $25^{\circ} \mathrm{C}$, through linear regressions on 5 points located in the steepest part of the curves. Please note: whether the t-axis assumes logarithimic scale won't affect the slope of $\gamma(t)$.

\section{Experimental}

\begin{tabular}{ll} 
Time & \multicolumn{1}{l}{$\boldsymbol{\gamma}$} \\
s & \multicolumn{1}{c}{$\mathrm{mJ} / \mathrm{m}^{2}$} \\
30 & 62.05569 \\
35 & 58.32862 \\
40.01 & 54.74696 \\
45.01 & 51.45897 \\
50.01 & 48.9553
\end{tabular}

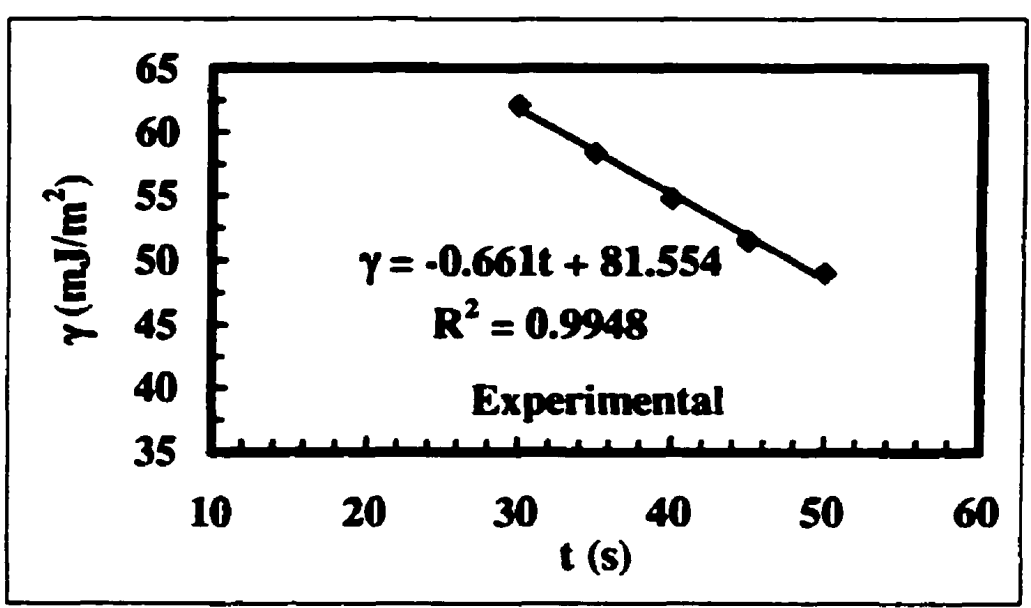

Langmuir Kinetics + SCF (Figure 3.2)

Time $\gamma$

s \begin{tabular}{rr}
\multicolumn{1}{c}{$\mathrm{mJ} / \mathrm{m}^{2}$} \\
31.6228 & 60.054 \\
35.4813 & 56.7233 \\
39.8107 & 53.5517 \\
44.6684 & 50.7849 \\
50.1187 & 48.5325
\end{tabular}

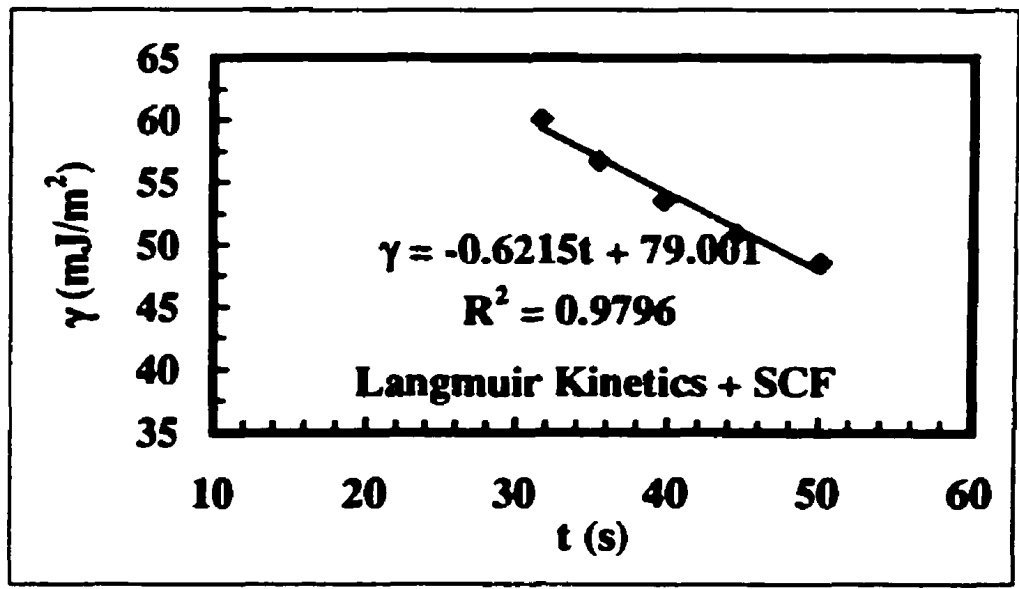


Langmuir Kinetics + Equation 3.7 (Figure 3.3)

t

$\mathbf{s}$

$\gamma$

$\mathrm{m} \mathbf{J} / \mathbf{m}^{2}$

$35.4813 \quad 67.3477$

$37.5 \quad 65.3063$

$39.8107 \quad 63.0968$

$44.6684 \quad 57.2878$

50.1187

51.502

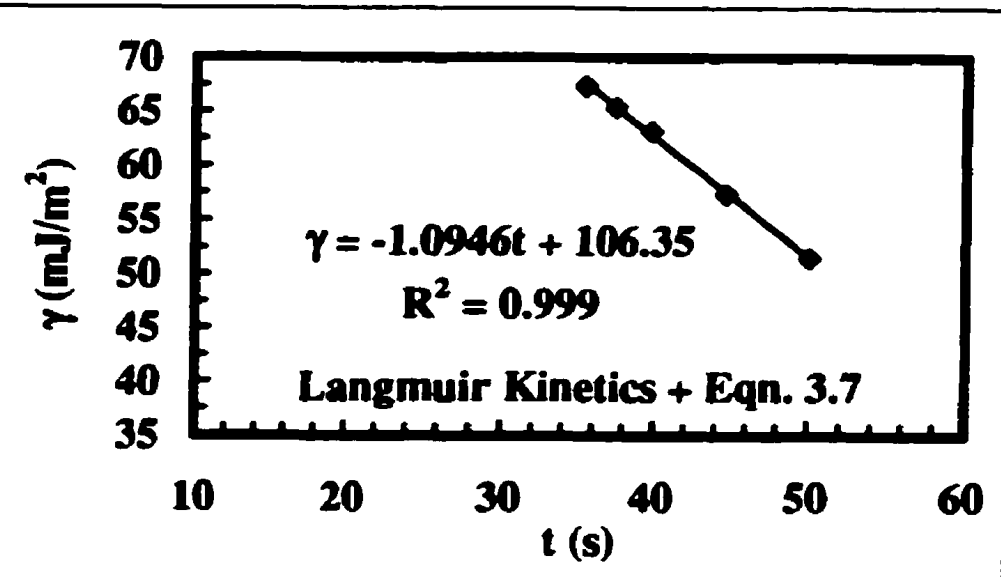

EC Model + SCF (Figure 3.4)

$\mathbf{t}$
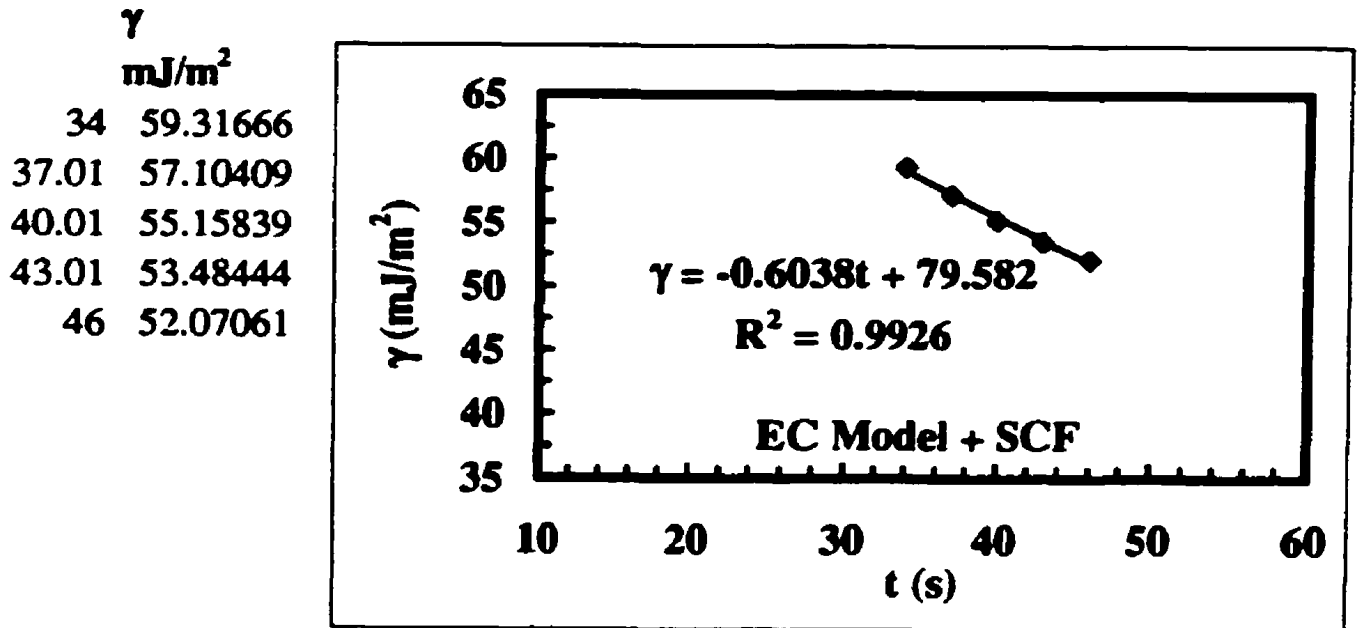


\title{
Chapter 4
}

\section{The Surface Tension of Aqueous Poly $(N$-isopropylacrylamide-co-acrylamide)}

\begin{abstract}
A series of poly( $N$-isopropylacrylamide-co-acrylamide) copolymers with $N$-isopropylacrylamide (NIPAM) to acrylamide (AM) molar ratios varying from $100: 0$ to $9: 91$ was synthesized and surface tensions, cloud point temperatures and enthalpies of phase separation were measured. At $25^{\circ} \mathrm{C}, 1 \mathrm{wt} \%$ poly $(N$ isopropylacrylamide) (PNIPAM) homopolymer has a surface tension of 41.94 $\mathrm{mJ} / \mathrm{m}^{2}$. Incorporation of $\mathbf{A M}$ moieties in the copolymer increased surface tension, which approached the limiting value of $65.3 \mathrm{~mJ} / \mathrm{m}^{2}$ for polyacrylamide solutions. The surface tension values of copolymer solutions were predicted from the surface tensions of the homopolymers applied to a one-parameter model analogous to the Margules model for the excess free energy of mixing. Heats of phase separation for the copolymer were less than expected compared with PNIPAM homopolymer. It was proposed that NIPAM moieties directly bonded to acrylamide did not contribute to the enthalpy of phase separation. Finally,
\end{abstract}


surface tension lowering kinetics were slower above the cloud point temperatures because at high temperatures the copolymers were present as colloidally dispersed particles which had to diffuse to the air/water interface, unwrap and spread to give an adsorbed layer.

Keywords: surface tension, cloud point, polymer adsorption, heat of phase separation, poly $(N$-isopropylacrylamide-co-acrylamide). 


\subsection{Introduction}

Temperature-sensitive poly( $N$-isopropylacrylamide) (PNIPAM) exhibits a lower critical solution temperature (LCST) between 30 and $35^{\circ} \mathrm{C}$ in an aqueous medium [1]. Copolymers of NIPAM and other vinyl monomer are also temperature sensitive $[2,3,4]$ and the copolymers have been evaluated for applications in enhanced/tertiary oil recovery $[5,6,7,8]$, optical "switching" devices [9], biotechnology $[10,11,12,13,14]$, drag reduction $[15,16]$, membrane technology [17] and chromatography [18].

Chiklis and Grasshoff [3] first reported the synthesis of poly(NIPAM-coAM) copolymers through free radical polymerization in water at room temperature using ammonium persulfate and sodium bisulfite as the redox catalyst. The monomer reactivity ratios found by Chiklis and Grasshoff were $r_{1}$ $=0.50$ for NIPAM and $r_{2}=1.00$ for AM. Later, Mumick and McCormick $[15,16]$ synthesized a series of poly(NIPAM-co-AM) copolymers by free radical polymerization in aqueous solutions at $30^{\circ} \mathrm{C}$ using potassium persulfate as the initiator. Those copolymers were characterized through turbidimetry, microcalorimetry, viscometry and composition study by elemental analysis and ${ }^{13} \mathrm{C}$ NMR and their drag reduction performance were investigated. Since acrylamide [AM] is more hydrophilic than NIPAM, the LCST of the copolymers increases with $\mathbf{A M}$ content. 
In previous work we have reported the dynamic surface tension behavior of aqueous PNIPAM homopolymers $[19,20]$ as a function of molecular weight and temperature. Interestingly, the equilibrium surface tension did not show a discontinuity at the LCST when plotted as a function of temperature [19], suggesting that polymer adsorbed at the air/water interface did not undergo a phase transition. Another interesting feature was that when a new interface was formed at a temperature above the LCST the resulting surface tension was low. Therefore PNIPAM, present as dispersed colloidal particles, diffused to the interface and unwrapped to give an adsorbed layer. The kinetics of surface tension lowering were modeled by simple mass transport models coupled to theoretical relationships between surface tension and the amount of adsorbed polymer [21]. PNIPAM can also be prepared as monodisperse, cross-linked colloidal microgels [22] and we have shown that the microgels adsorbed onto the air/water interface can lower surface tension to about the same extent as linear PNIPAM (see Chapter 5).

In this work, we have extended our investigation of PNIPAM at the air/water interface by reporting the surface tension characteristics of a series of poly(NIPAM-co-AM) copolymers. In addition to surface tension kinetics, cloud point temperature (CPT) and heats of phase separation are reported and compared with previously published results. 


\subsection{Experimental}

\subsubsection{Preparation of the polymers}

Polyacrylamide (PAM) with a molecular weight of $5 \times 10^{6}$ was obtained from Nalco Canada Inc. The preparation and characterization of PNIPAM homopolymer (L-6B) was reported previously [19]. NIPAM (Kodak Co.) was recrystallized twice from toluene/hexane. AM (Aldrich), initiator ammonium persulfate (Aldrich) and sodium bisulfite (Aldrich) were used as received.

Table 4. 1 The feed ratios, copolymer NIPAM fractions (mol\%), molecular weights and distributions of the copolymers prepared in this work.

\begin{tabular}{|c|c|c|c|c|c|}
\hline Sample & $\begin{array}{l}\text { Feed Molar Ratio } \\
\text { NIPAM/AM }\end{array}$ & $\begin{array}{l}\text { NTPAM in Copolymer } \\
(\mathrm{mol} \%)\end{array}$ & $\begin{array}{l}\bar{M}_{w} \\
\left(\times 10^{-5}\right)\end{array}$ & $\begin{array}{c}\bar{M}_{n} \\
\left(\times 10^{-5}\right)\end{array}$ & $\frac{\bar{M}_{w}}{\bar{M}_{n}}$ \\
\hline 2.68 & $100 / 0$ & 100 & 5.47 & 3.11 & 1.76 \\
\hline coss0s & $95 / 5$ & 93.9 & & & \\
\hline C09307 & $92.7 \pi .4$ & 89.8 & 2.71 & 1.06 & 2.54 \\
\hline c09010 & $90 / 10$ & 86.9 & 3.80 & 1.71 & 2.22 \\
\hline C08614 & $86.3 / 13.7$ & 81.8 & 2.86 & 1.16 & 2.46 \\
\hline C07624 & $75.9 / 24.1$ & 72.9 & 3.22 & 1.48 & 2.17 \\
\hline$C 07030$ & $70 / 30$ & 68.4 & 4.15 & 1.95 & 2.12 \\
\hline CO6040 & $60 / 40$ & 58.4 & 3.12 & 1.56 & 2.01 \\
\hline COS050 & $50 / 50$ & 48.5 & 4.35 & 2.10 & 2.07 \\
\hline COA060 & $40 / 60$ & 39.1 & 3.35 & 1.58 & 2.13 \\
\hline$C 03070$ & $30 / 70$ & 29.6 & 2.27 & 1.09 & 2.08 \\
\hline CO2080 & $20 / 80$ & 20.0 & 2.50 & 1.16 & 2.15 \\
\hline co10so & $10 / 90$ & 9.0 & 4.42 & 1.90 & 2.33 \\
\hline
\end{tabular}


Poly(NIPAM-co-AM) polymers with a NIPAM to AM molar ratio varying from 95:5 to 10:90 were synthesized by free radical polymerization. The feed ratio for each copolymer is listed in Table 4. 1. In a typical polymerization, 100 $\mathrm{ml}$ of a $0.88 \mathrm{M}$ (total monomer concentration) solution of the monomers dissolved in Milli $\mathrm{Q}$ water was placed in a $250 \mathrm{ml}$ one-necked round bottom flask fitted with a magnetic stirrer. After $15 \mathrm{~min}$ of purging with nitrogen at room temperature, $5 \mathrm{ml}$ ammonium persulfate solution was added followed immediately by $5 \mathrm{ml}$ sodium bisulfite solution under a nitrogen blanket. The final total monomer concentration was $0.8 \mathrm{M}$ and the initial concentrations of both ammonium persulfate and sodium bisulfite were $2.93 \times 10^{-4} \mathrm{M}$. The polymerization mixtures were stirred for 10 hours at room temperature.

Copolymers with NIPAM contents greater than 50\% were precipitated out in warm saturated $\mathrm{NaCl}$ solution whereas the other copolymers were precipitated out in acetone. All the copolymers and the two homopolymers were further purified by exhaustive dialysis using membrane tubing of $12,000-14,000$ M.W cutoff.

\subsubsection{Characterizations of the copolymers}

Molecular weight and distribution. The weight average and number average molecular weight $\left(\bar{M}_{w}\right.$ and $\left.\bar{M}_{n}\right)$ and the molecular weight distribution $\left(\bar{M}_{n}\right)$ were measured by a GPC equipped with a Waters 510 HPLC pump, 
coupled with Waters 410 differential refractometer and Waters 486 tunable absorbance detector. The column was maintained at a temperature of $30.0 \pm 0.1$ ${ }^{\circ} \mathrm{C}$. The eluant was $0.1 \mathrm{~N} \mathrm{NaNO}$ aqueous solution running at $0.7 \mathrm{ml} / \mathrm{min}$. Polyethylene oxide standards were used for calibration. The results are summarized in Table 4. 1.

${ }^{1} H$ NMR Composition Analysis. The proton spectra of the copolymers in $2 w t \% D_{2} O$ solutions were obtained on a Bruker AC 200 NMR spectrometer (Bruker Co.) operating at $200.1 \mathrm{~T}$. A typical ${ }^{1} \mathrm{H}$ NMR spectrum of a poly(NIPAM-co-AM) copolymer was shown elsewhere (see Appendix 4.1). The composition of the copolymers were determined through integration of the methyl proton peak area $(A)$, the methylene proton peak area $(B)$, and the methyne proton peak area $(C)$. The NIPAM mole fraction in a copolymer was $\frac{0.5 A}{B+C}$ and the AM content was $\left(1-\frac{0.5 A}{B+C}\right)$.

\subsubsection{Cloud Points}

About $0.05 \mathrm{ml}$ of $7 \mathrm{wt} \%$ aqueous solution was injected into a $1.5 \sim 1.8 \times 90$ mm Kimax-51 (Kimble Products) capillary. The open ends of the capillaries were fused with a torch and, after cooling, the capillaries were immersed into a silicone oil bath, which was heated at $1{ }^{\circ} \mathrm{C} / \mathrm{min}$ from 24 to $130{ }^{\circ} \mathrm{C}$. The temperature at which the first turbidity in a solution appeared was observed visually and 
recorded as the cloud point.

\subsubsection{Heat of Phase Separation ( $\Delta H)$}

Aqueous copolymer solutions of 7 wt\% were sealed within weighted helmet aluminum pans. A 910 Differential Scanning calorimeter (TA Instruments) with indium calibration was used. The samples were scanned at 5.00 ${ }^{\circ} \mathrm{C} / \mathrm{min}$ from 5 to $100^{\circ} \mathrm{C}$. The heat of phase separation was measured as a function of copolymer NIPAM fraction and analyzed by V4.0 General Analysis (DuPont). A typical DSC trace of a poly(NIPAM-co-AM) is available elsewhere (see Appendix 4.2).

\subsubsection{Dynamic Surface Tension Measurement}

The dynamic surface tensions of aqueous copolymer solutions were measured by the pendent drop method using the axisymmetric drop shape analysis (ADSA-P). The ADSA-P method, developed by Neumann and coworkers, fits the shape of a pendent drop to the theoretical drop profile given by the Laplace equation to give the surface tension. The error limits of these measurements are approximately $0.1 \mathrm{~mJ} / \mathrm{m}^{2}$ or less. Pendent drops were formed on the tip of a Teflon capillary with an outside diameter of 0.1 in. and inside diameter of 0.076 in. (Chromatographic Specialties Inc.). The pendent drop was enclosed in a

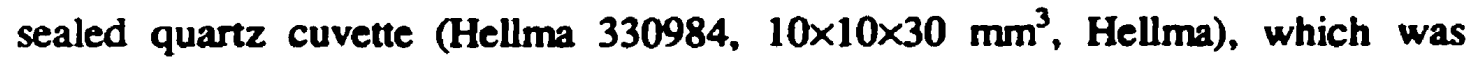
mounted in a Model 100-07 environmental chamber (Rame Hart Inc.). The 
temperature control was $\pm 0.1{ }^{\circ} \mathrm{C}$. Further details of the apparatus and methodology can be found elsewhere $[23,24]$. The water used as solvent in preparing the solutions had a surface tension of $72.20 \mathrm{~mJ} / \mathrm{m}^{2}$ at $25^{\circ} \mathrm{C}$, which was measured using the above-mentioned ADSA-P technology and in good agreement with $72.14 \mathrm{~mJ} / \mathrm{m}^{2}$ given by Jasper [25]. 


\subsection{Results}

A series of poly(NIPAM-co-AM) copolymers was prepared and purified and their composition and molecular weight properties are summarized in Table 4. 1. The NIPAM content of the copolymers measured by NMR was 1 2 mol\% less than the feed. This observation is consistent with the work of Chiklis and Grasshoff, which showed that AM is more reactive than NIPAM [3]. A more detailed analysis of copolymer microstructure is given in the discussion section.

The molecular weight of the copolymers changed from $2.71 \times 10^{5}$ to $4.35 \times 10^{5}$ for $\bar{M}_{w}$ and from $1.06 \times 10^{5}$ to $2.10 \times 10^{5}$ for $\bar{M}_{n}$. The corresponding $\frac{\bar{M}_{w}}{\bar{M}_{n}}$ varied from 2.01 to 2.54 .

\subsubsection{Cloud Point Temperature (CPT)}

The CPTs of 7 wt\% aqueous solutions were measured visually and are plotted in Figure 4. 1 as a function of the mole fraction of NIPAM in the copolymers $\left(f_{\text {NIPAM }}\right)$. The CPT increased with increasing temperature. The CPT for copolymers with less than 45 mol\% NIPAM could not be measured. Also shown in the figure are the results of Chiklis and Grasshoff [3] - although their concentrations and molecular weights were different, the CPT values were similar. 


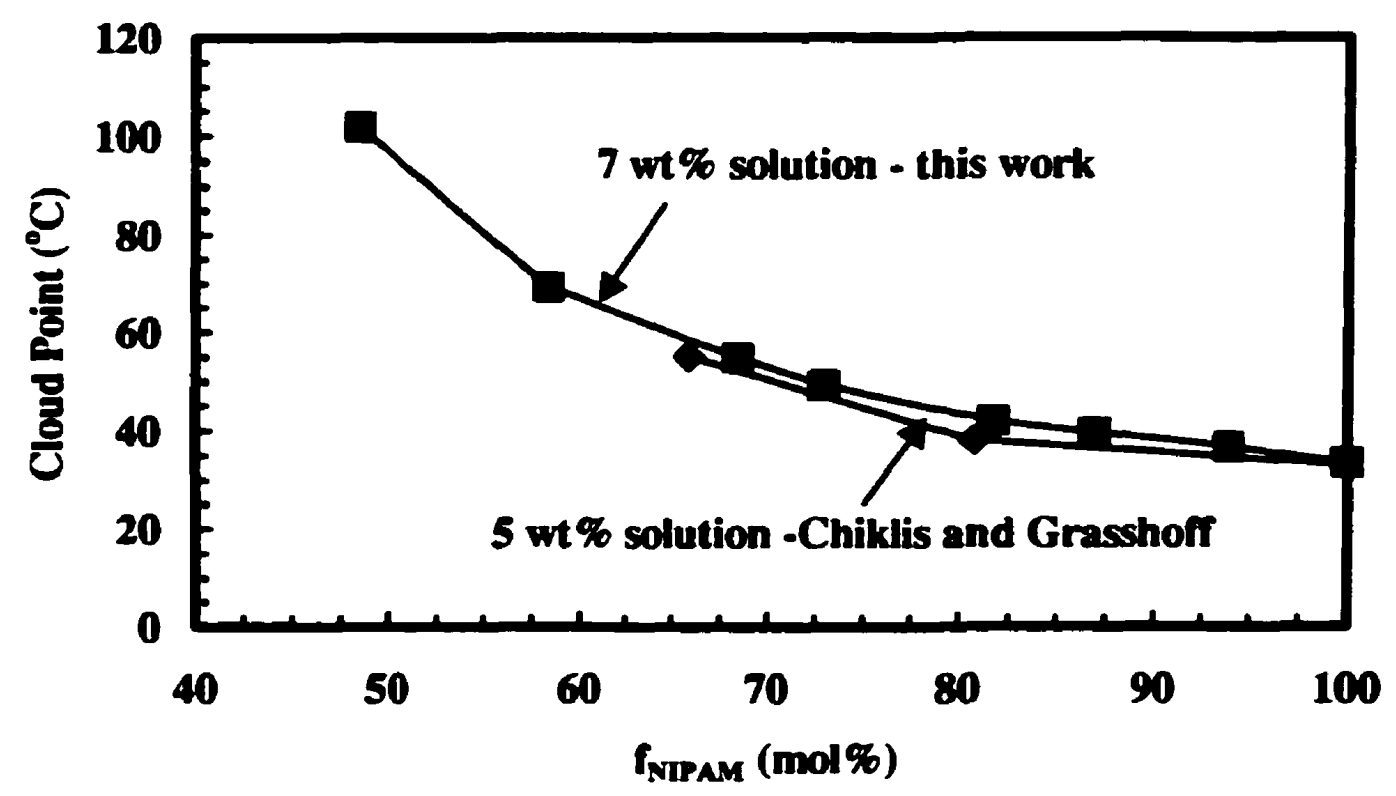

Figure 4. 1 The cloud point temperature as functions of the mole percent of NIPAM in the copolymers. The published data are shown for comparison.

\subsubsection{Heat of Phase Separation}

The heat effects associated with copolymer phase separation were measured by differential scanning calorimetry. Endothermic peaks were found when the aqueous copolymer solutions were heated through the CPT. The results are summarized in Figure 4. 2, where the enthalpy of phase separation is plotted as a function of the mole fraction of NIPAM moieties in the copolymer - see the curve labeled "Per mole of 'Interior' IP groups". The enthalpies are expressed as $\mathrm{kJ}$ per mole of isopropyl groups because heat effects have been explained by the water association with these hydrophobic groups [26]. The enthalpies increased linearly with NIPAM contents from 60 to 100 mol\%. Heat effects associated with 
phase separation for copolymers with less than $68 \mathrm{~mol} \%$ could not be measured by our instrument. The enthalpy change for PNIPAM homopolymer was 5.76 $\mathrm{kJ} / \mathrm{mol}$ of NIPAM groups which falls between reported values of $4.73 \mathrm{~kJ} / \mathrm{mol}$ of NIPAM groups by Fujishige et al [27] and $6.28 \mathrm{~kJ} / \mathrm{mol}$ of NIPAM groups by Schild and Tirrell [28]. If the heat effect is only associated with the isopropyl groups, one would expect the plot in Figure 4.2 to be a horizontal line. Instead, it has a large positive slope - this will be addressed in the discussion section, together with an explanation of the second curve in the figure.

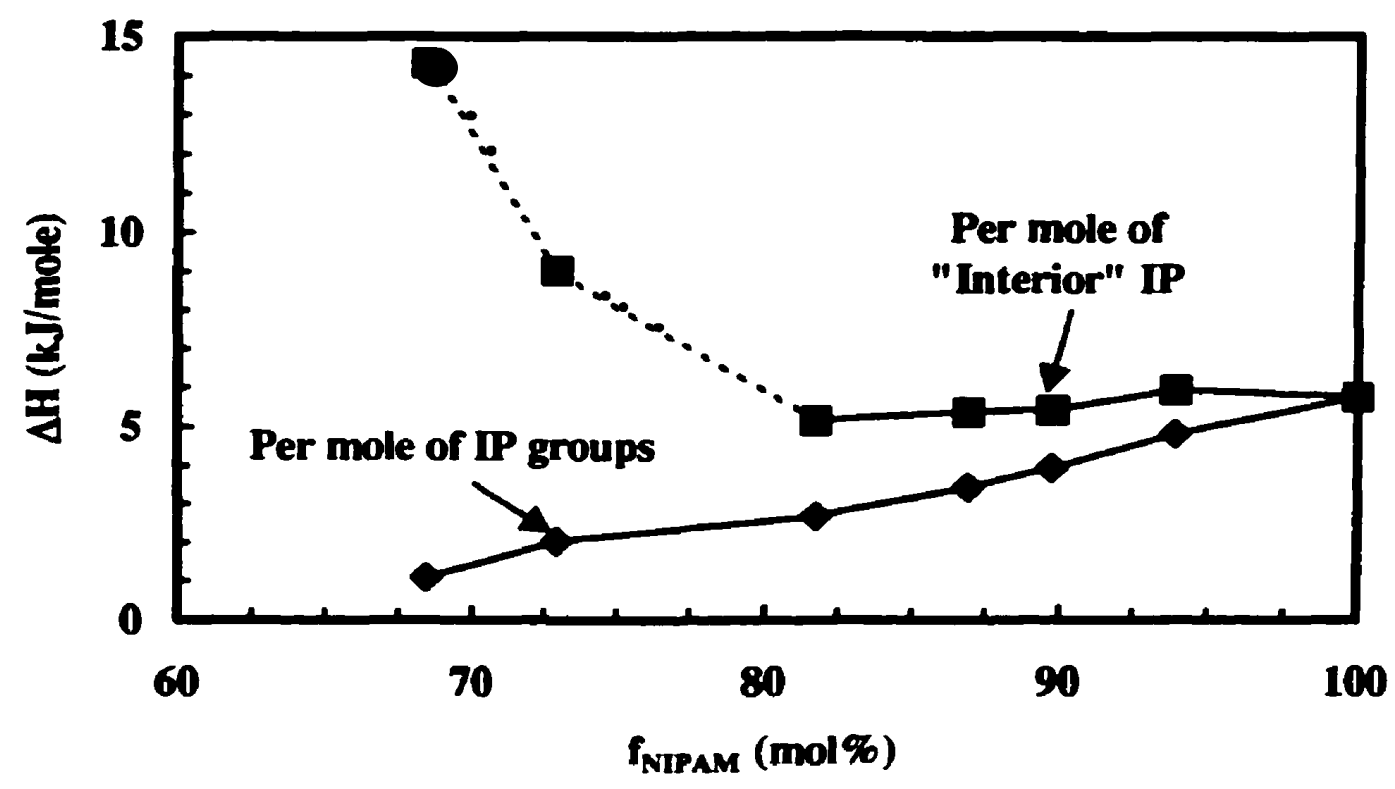

Figure 4. 2 The heat of phase separation as a function of copolymer NIPAM fraction $\left(f_{\text {NIPAM }}\right.$ mol\%).

\subsubsection{Dynamic Surface Tension}

The dynamic surface tensions of the $1 \mathrm{wt} \%$ copolymer solutions at $25^{\circ} \mathrm{C}$ 
are plotted as functions of the pendent drop age in Figure 4. 3. Polyacrylamide (PAM) is slightly surface active - the surface tension of 1 wt\% PAM aqueous solution stabilized at $69.15 \mathrm{~mJ} / \mathrm{m}^{2}$ at $25^{\circ} \mathrm{C}$ after $80 \mathrm{~min}$. The first two digits in the copolymer labels give the approximate mole fraction of NIPAM (see Table 4. 1). The surface tension values in Figure 4. 3 systematically decreased with increasing NIPAM content in the copolymer with the limiting value of 41.94 $\mathrm{mJ} / \mathrm{m}^{2}$ for PNIPAM homopolymer (L-6B).

The kinetics of surface tension lowering was rapid for concentrated (1 wt\%) solutions shown in Figure 4. 3. Meso-equilibrium (i.e. steady-state) surface tension values were obtained within 100 seconds and the first recorded values were close to the meso-equilibrium values. Kinetic effects are more significant at lower polymer concentrations. Shown in Figure 4.4 are dynamic surface tension results obtained with 100 ppm solutions. At $25^{\circ} \mathrm{C}$ surface tension decreased significantly in the first 10 seconds. In previous work we have shown that one must have PNIPAM concentrations less than $50 \mathrm{ppm}$ to achieve surface tension lowering over 100 seconds [20]. The kinetics were much slower at $40{ }^{\circ} \mathrm{C}$ because the mass transport of colloidally dispersed, phased separated polymers was slower than the diffusion of dissolved polymer molecules at lower temperatures. 


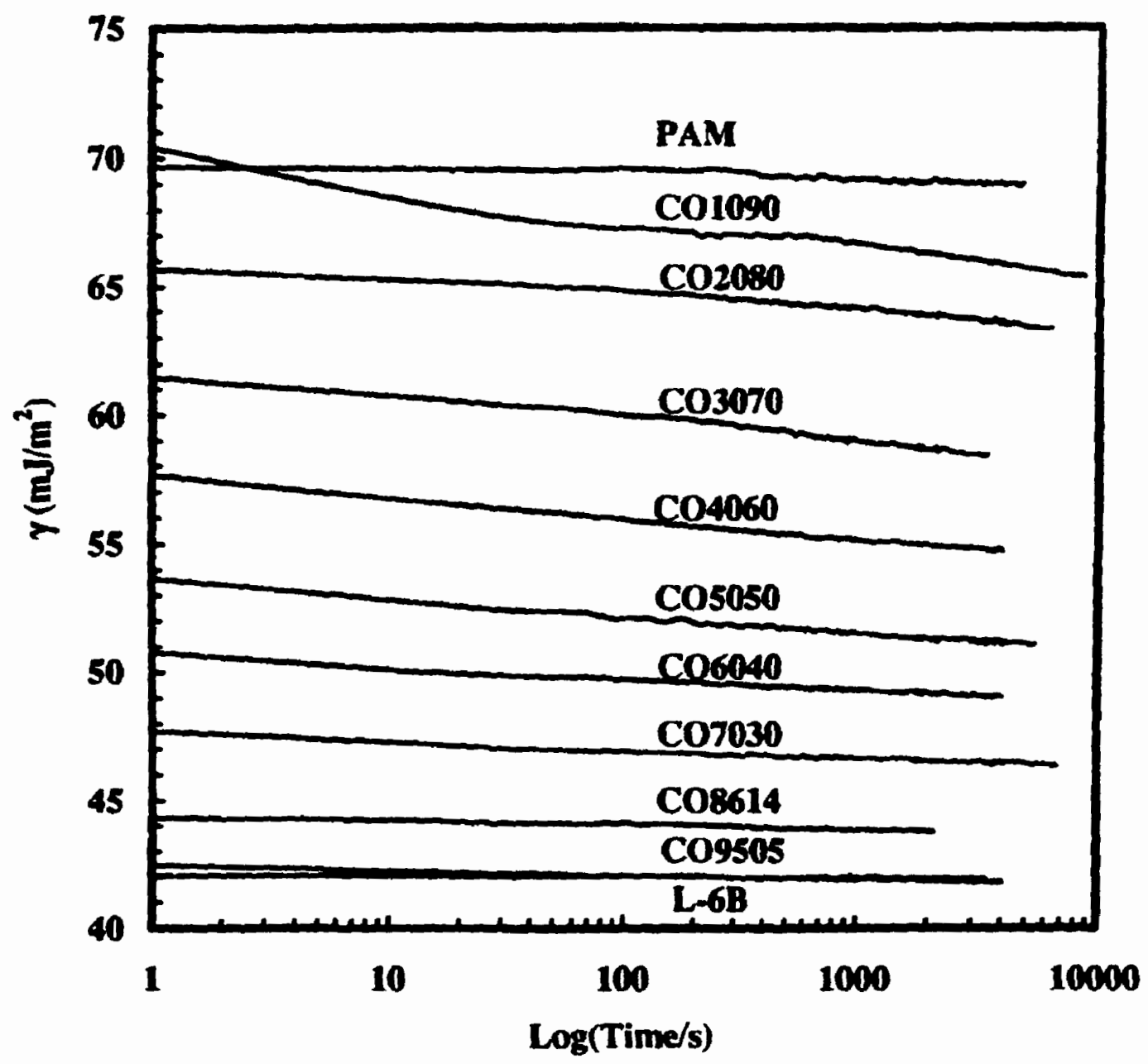

Figure 4.3 The dynamic surface tension of $1 \mathrm{wt} \%$ copolymer solutions at $25^{\circ} \mathrm{C}$. 


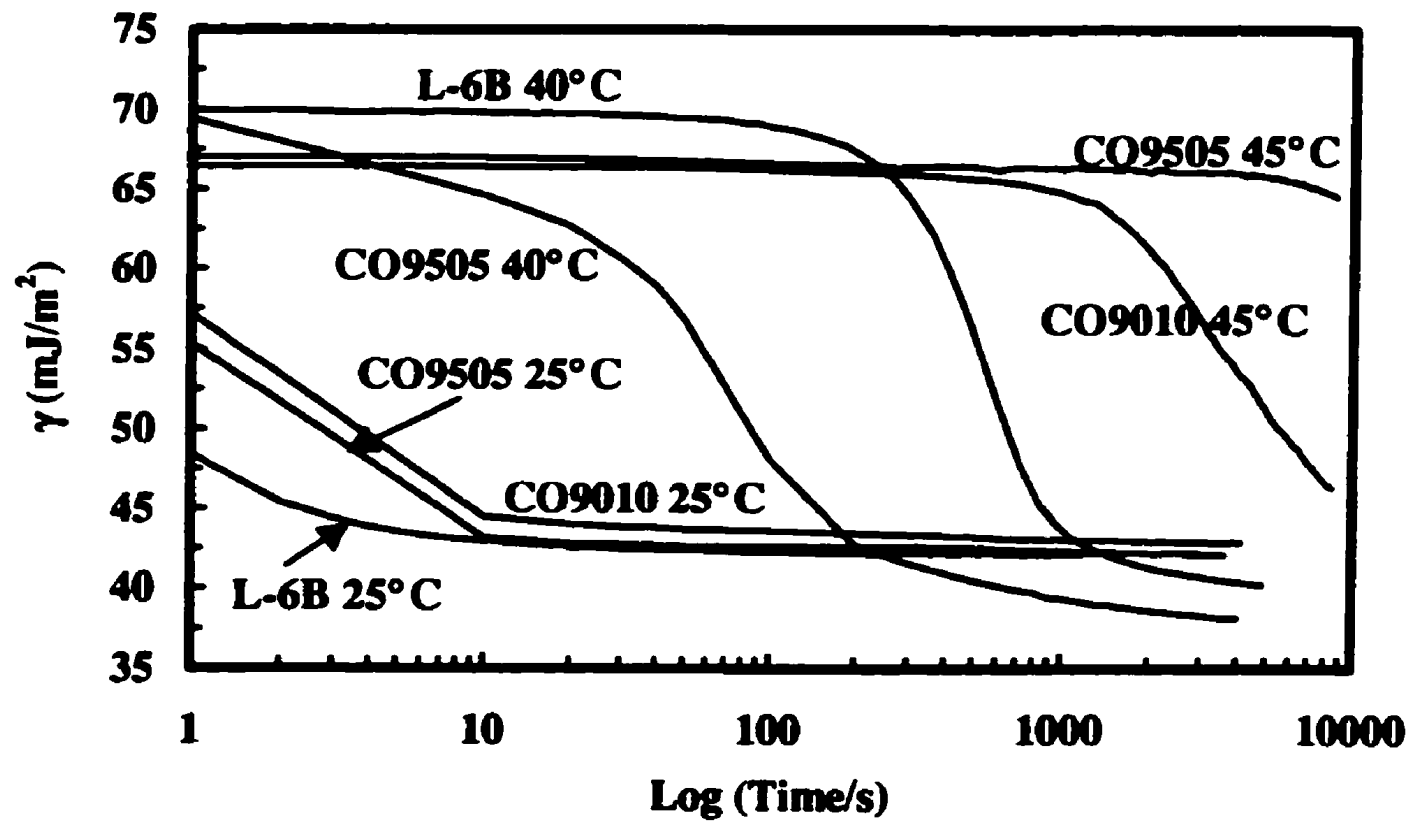

Figure 4.4 The dynamic surface tension of $100 \mathrm{ppm}$ copolymer solutions at 25,40 and $45^{\circ} \mathrm{C}$. 


\subsection{Discussion}

Polyacrylamide does not lower the surface tension of water whereas PNIPAM does. Although unproven, it seems reasonable to propose that the hydrophobic $N$-isopropyl groups on the NIPAM moieties concentrate at the air/water interface thus lowering surface tension. The results in Figure 4. 3 show that the more the NIPAM groups present in polymer, the lower the surface tension. The meso-equilibrium surface tension values from Figure 4. 3 are shown as a function of NIPAM content in Figure 4. 5 (the data points). Also, shown is a line (labeled ideal) between the surface tensions values for PAM and PNIPAM solutions which is given by the following equation.

$$
\gamma_{\text {ideal }}=\gamma_{M M}-f_{\text {NPAM }}\left(\gamma_{M M}-\gamma_{\text {MPAM }}\right)
$$

Equation 4.1

where $\gamma_{A M}$ is the surface tension of PAM solution, $\gamma_{\text {NIPAM }}$ is the surface tension of PNIPAM solution and $f_{\text {NIPAM }}$ is the mole fraction of NIPAM moieties in the copolymer. The experimental surface tension values were lower than the corresponding ideal values. An excess surface tension can be defined as:

$$
\gamma_{E x}=\gamma-\gamma_{\text {ideal }}=\gamma-\gamma_{\text {MM }}+f_{\text {NPMM }}\left(\gamma_{\text {AM }}-\gamma_{\text {MPMM }}\right)
$$

Equation 4.2

where $\gamma_{E X}$ is the excess surface tension and $\gamma$ is the actual surface tension. 


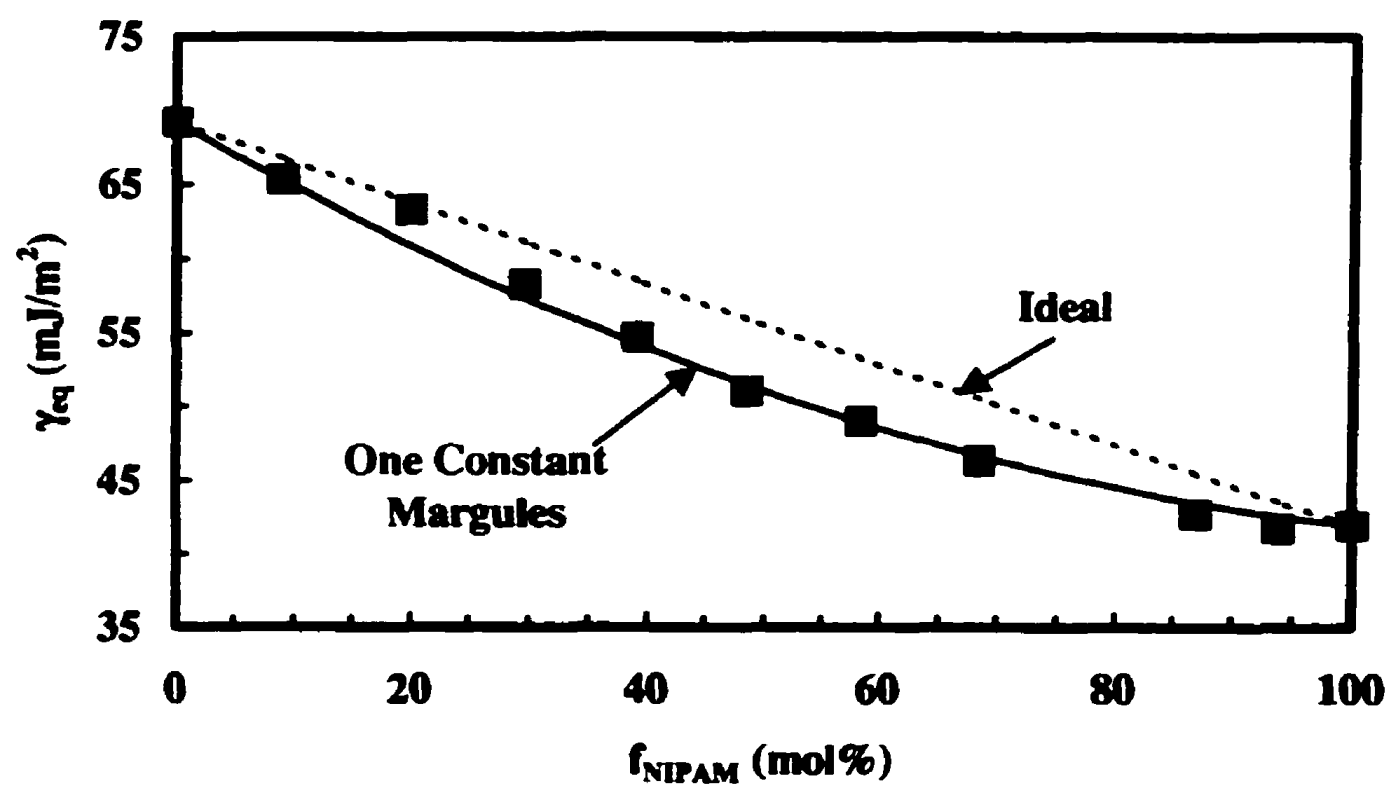

Figure 4.5 The experimental meso-equilibrium surface tension (D) of the 1 wt\% aqueous copolymer solutions at $25^{\circ} \mathrm{C}$ as a function of copolymer NIPAM fraction (mol\%), the equilibrium surface tension (solid line) from Margules model (Equation 4.4) and the ideal equilibrium surface tension (dotted line) as defined by Equation 4.1.

One of the simplest treatments for excess quantities in solution thermodynamics is the one-constant Margules model. This model can be applied to predict excess surface tension giving the following relationship

$$
\gamma_{E X}=f_{\text {NIPN }}\left(1-f_{\text {NIPM }}\right) A \quad \text { Equation } 4.3
$$

where $A$ is a constant which is independent of polymer composition. Experimental excess surface tension values are shown as the data points in Figure 4. 6. The solid line was computed from Equation 4.3 with $A$ chosen to be -18 $\mathrm{mJ} / \mathrm{m}^{2}$. The maximum excess surface tensions corresponded to a NIPAM mole 
fraction of 0.5 which are the conditions giving the maximum number of $\mathrm{AM}$ NIPAM bonds. Thus, non-ideality seems to be associated with AM-NIPAM interactions along the copolymer chain. From a molecular structure perspective, the isopropyl groups on NIPAM seem to be able to compensate for the presence of neighboring AM groups at the air/water interface.

Combining Equations 4.2 and 4.3 gives the following expression relating copolymer surface tension to the experimental surface tension of PAM and PNIPAM.

$$
\gamma=\gamma_{\text {MM }}-f_{\text {NIPM }}\left(\gamma_{\text {MM }}-\gamma_{\text {NIPM }}\right)+x_{\text {NPPM }} x_{\text {MM }} A
$$

Equation 4.4

The line labeled "One-Constant Margules" in Figure 4. 5 was calculated from Equation 4.4. The simple model for excess surface tension gives a good fit to the experimental data. 


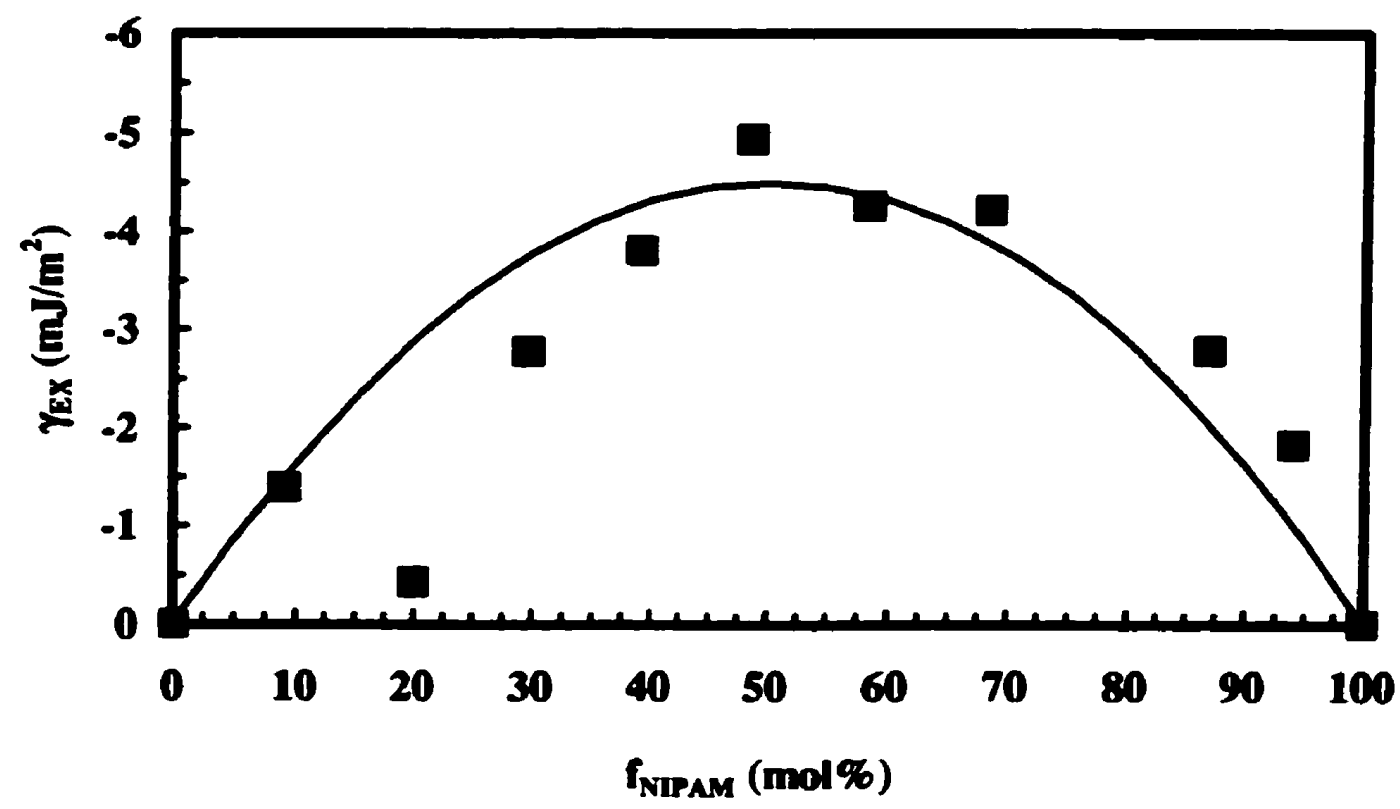

Figure 4.6 Excess surface tension as a function of the mole fraction of NIPAM in the copolymer.

Heating the copolymers through the cloud point temperature caused phase separation, which was endothermic. Heskins and Guillet were the first to report this for PNIPAM [29]. The enthalpies of phase separation per mole of isopropyl group in the copolymers were not independent of copolymer concentration. Indeed, the results in Figure 4. 2 (curve labeled "Per mole of IP groups") show a dramatic decrease in the heat effect with a decreasing NIPAM (or IP) content. This in turn suggests that the heat effect is not associated with isolated isopropyl groups but instead is associated with PNIPAM blocks in the copolymer chain. The copolymer microstructure can be inferred from the polymerization kinetics.

Chiklis and Grasshoff [3] reponed the monomer reactivity ratios for 
NIPAM as $r_{1}=0.5$ and for AM as $r_{2}=1.0$ by the method of Fineman and Ross [30]. Using these reactivity ratio values, the mean sequence length for NIPAM and AM sequences were calculated by the method of Pyun [31] assuming the copolymerizations were Markov processes (see Appendix 4.3). The results, summarized in Table 4. 2, show that with more than $15 \mathrm{~mol} \%$ AM, the mean sequence length of the NIPAM blocks dropped to near two.

A PNIPAM sequence in a copolymer molecule has two exterior NIPAM moieties which are each bonded to one AM moiety. The remainder of the NIPAM moieties are "interior". In an attempt to further clarify the meaning of the enthalpy of phase separation, we assumed that only "interior isopropyl groups" contribute to the heat effect. The enthalpy change per interior isopropyl group can be calculated as:

$$
\Delta H_{\text {IIP }}=\Delta H_{\text {IP }} \frac{M S L}{M S L-2}
$$

\section{Equation 4.5}

where $\Delta H_{l, P}$ is the enthalpy change per interior isopropyl group, $\Delta H_{l p}$ is the enthalpy change per interior plus exterior isopropyl groups, and MSL is the mean sequence length for NIPAM sequences (see Table 4. 2) in the copolymer. The enthalpy results expressed per mole of interior isopropyl groups are shown as the top curve in Figure 4. 2. From 80 to 100 mole percent NIPAM, the enthalpy per mole of interior isopropyl groups was independent of composition suggesting some validly in the analysis. However, the final two points corresponding to a mean sequence length of 2.6 and 2.2 , gave high values. 
Table 4. 2 The mean sequence lengths for the copolymers.

\begin{tabular}{|c|c|c|}
\hline \multirow[t]{2}{*}{ Sample } & \multicolumn{2}{|c|}{$\begin{array}{l}\text { Mean Sequence } \\
\text { Length (comonomer unit) }\end{array}$} \\
\hline & NIPAM & $\mathbf{A M}$ \\
\hline Coss0s & 10.5 & 1.05 \\
\hline C09307 & 7.29 & 1.08 \\
\hline coso10 & 5.5 & 1.11 \\
\hline C08614 & 4.15 & 1.16 \\
\hline C07624 & 2.57 & 1.32 \\
\hline C07030 & 2.17 & 1.43 \\
\hline Cosia & 1.75 & 1.67 \\
\hline Cososo & 1.50 & 2.00 \\
\hline c04060 & 1.33 & 2.50 \\
\hline $\operatorname{co3070}$ & 1.21 & 3.33 \\
\hline CO2080 & 1.14 & 4.50 \\
\hline $\cos 090$ & 1.10 & 10.00 \\
\hline
\end{tabular}

In summary, non-ideality in both surface tensions and enthalpies of phase separation when considered as functions of the copolymer content, seems to be proportional to the fraction of NIPAM groups which were directly bonded to at least one AM moiety. Specifically, the surface tension values associated with AM groups bonded to NIPAM were lower than predicted by the ideal case. Similarly, the enthalpy of phase separation was also lower than expected in proportion to the fraction of NIPAM having AM-NIPAM links. 


\subsection{Conclusion}

1. Poly(NIPAM-co-AM) lowers the surface tension of water. PNIPAM homopolymer gives the greatest surface tension lowering and the higher the NIPAM content, the lower the surface tension.

2. The surface tension of copolymer solutions can be predicted from the surface tensions of the homopolymers applied to a one-parameter model (Equation 4.4) analogous to the Margules model for the excess free energy of mixing.

3. Surface tension lowering kinetics were slower above the cloud point temperatures because at high temperatures the copolymers were present as colloidally dispersed particles which had to diffuse to the air/water interface, unwrap and spread to give an adsorbed monolayer.

4. Heats of phase separation for the copolymer were less than expected compared with PNIPAM homopolymer. It was proposed that NIPAM moieties directly bonded to acrylamide did not contribute to the enthalpy of phase separation. 


\subsection{Acknowledgements}

The authors acknowledge Professor A. Wilhelm Neumann and Dr. Yu Hing Daniel Kwok at University of Toronto, for allowing us to use their surface tension apparatus. The authors also thank the Canadian Natural Science and Engineering Research Council for funding. 


\subsection{References}

1 Schild, H. G., Prog. Polym. Sci., 17, 163 (1992).

2 Casolaro, M., Polymer, 38, 4215 (1997).

3 Chiklis, C. K. and Grasshoff, J. M., J. Polym. Sci.: Part A-2, 8, 1617 (1970).

4 Baines, F. L.; Billingham, N. C. and Armes, S. P., Macromolecules, 29, 3416 (1996).

5 Siano, D. B.; Bock, J.; Myer, P. and Valint, P. L., ACS. PMSE Prepr., 57, 609 (1987).

6 Bock, J.; Siano,D. B.; Valint, P. L. and Pace, S. J., ACS. PMSE Prepr. 57, 487 (1987).

7 Nagarajan, R., Polym. Prepr. (Am. Chem. Soc., Div. Polym. Chem.), 22 (2), 33 (1987).

8 Khune,G. D.; Donaruma, L. G.; Hatch, M. J.; Kilmer, N. H.; Shepitka, J. S. and Martin, F. D., Polym. Prepr. (Am. Chem. Soc., Div. Polym. Chem.), 22 (2), 76 (1981).

9 Kungwatchakun, D. and Irie, M.; Makromolek. Chem., Rapid Commun., 9, 243 (1988).

10 Stayton, P. S.; Shimoboji, T.; Long, C.; Chilkoti, A.; Chen, G.; Harris, J. M.; and Hoffman, A. S., Nature, 378, 472 (1995).

11 Chen, G. and Hoffman, A. S., Nature, 373, 49 (1995).

12 Chen, G. and Hoffman, A. S., Macromol. Rapid Commun., 16, 175 (1995).

13 Chen, G. and Hoffman, A. S., J. Biomater. Sci. Polymer Edn., 5, 371 (1994).

14 Yang, H. J.; Cole, C.; Monji, N. and Hoffman, A. S., J. Polym. Sci.: Part A: Polym Chem., 28, 219 (1990).

15 Mumick, P. S. and McCormick, C. L., Polymer Engineering and Science, 34, 1419 (1994). 
16 Mumick, P. S.; Hester, R. D. and McCormick, C. L., Polymer Engineering and Science, 34, 1429 (1994).

17 Nonaka, T.; Hashimoto, K. and Kurihara, S., J. Applied Polym. Sci., 66, 209 (1997).

18 Ivanov, A. E.; Zhigis, L. S.; Kurganova, E. V.; and Zubov, V. P., J. Chromatography A, 776, 75 (1997).

19 Zhang, Ju and Pelton, R., Langmuir, 12, 2611 (1996).

20 Zhang, Ju and Pelton, R., "The dynamic behavior of poly( $N$ isopropylacrylamide) at the Air/Water Interface", accepted by Colloids and Surfaces.

21 Zhang, Ju and Pelton, R., "The application of polymer adsorption models to dynamic surface tension", submitted to Langmuir.

22 Pelton, R.H. and Chibante, P., Colloids and Surfaces, 20, 247(1986).

23 Rotenberg, Y.; Boruvka, L.; Neumann, A. W., J. Colloid Interface Sci., 93, 169 (1983).

24 Kwok, Daniel Yu Hing, Masters Thesis, University of Toronto, 1994.

25 Jasper, J. J., J. Physical and Chemical Reference Data, 1, 948 (1972).

26 Schild, H. G. and Tirrell, D. A., J. Phys. Chem., 94, 4352 (1990).

27 Fujishige, S.; Kubota, K. and Ando, I., J. Phys. Chem. 93, 3311 (1989).

28 Schild, H. G. and Tirrell, D. A., J. Phys. Chem., 94, 4352 (1990).

29 Heskins, M. and Guillet, J. E., J. Macromol.Sc.-Chem., A2, 1441 (1968).

30 Fineman, M. and Ross, S., J. Polym. Sci., 5, 259 (1950).

31 Pyun, C. W., J. Polym. Sci.: Part A-2, 8, 1111 (1970). 
Appendix 4.1 Examples of ${ }^{1} \mathbf{H}$ NMR Spectrum of Copolymers 


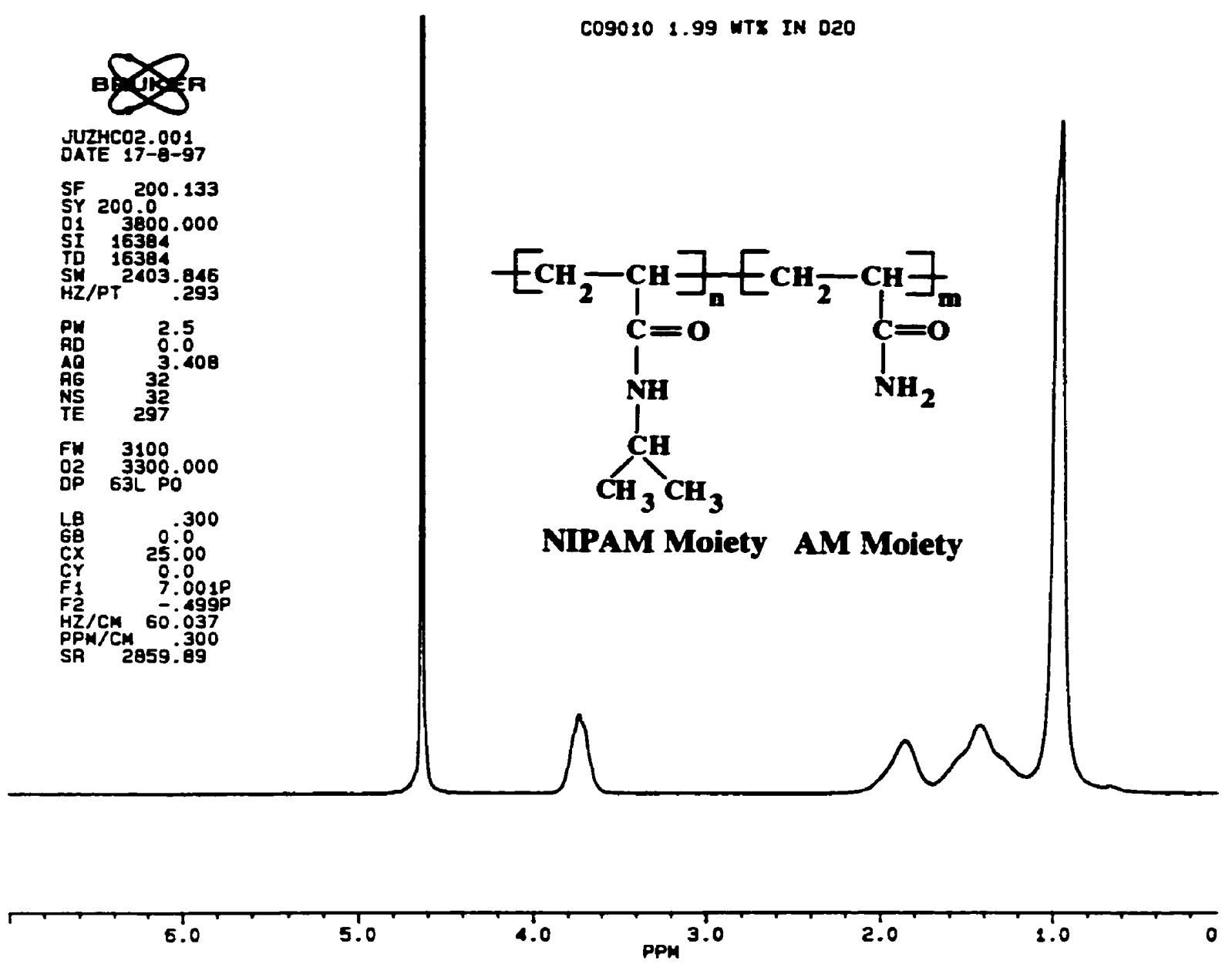




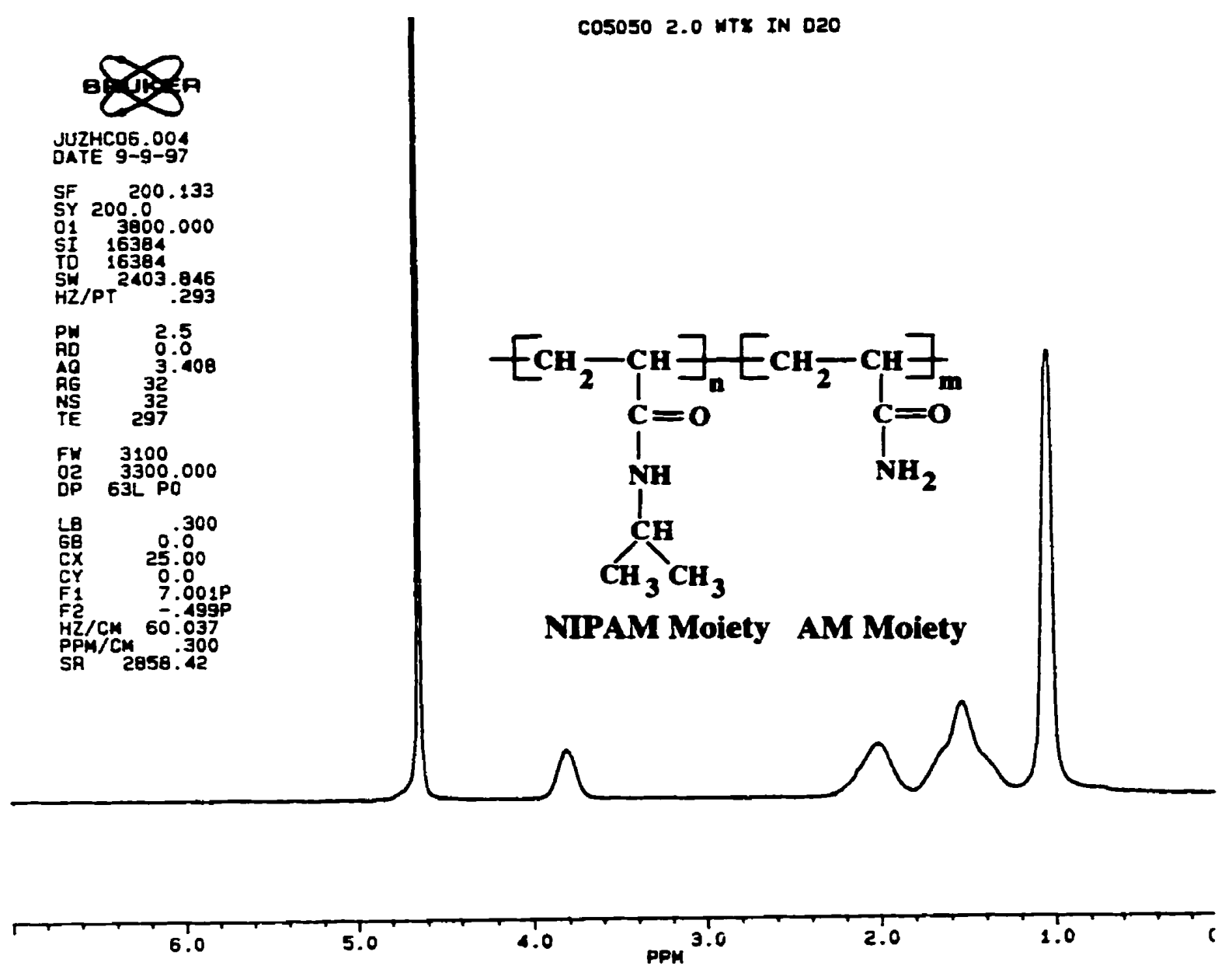


Appendix 4.2 Examples of DSC Traces 
Samp le: L-6B $7.00 \mathrm{WTX}$ IN H2O S1ze: $1.5981 \mathrm{ng}$ Method: JU

Comment: $5.00 \mathrm{C} / \mathrm{MIN}$ TO $100 \mathrm{C}$ $\square S C$ F11e: A: L68.001

Operator: N

Pun Date: 26-Aug-97 21: 25

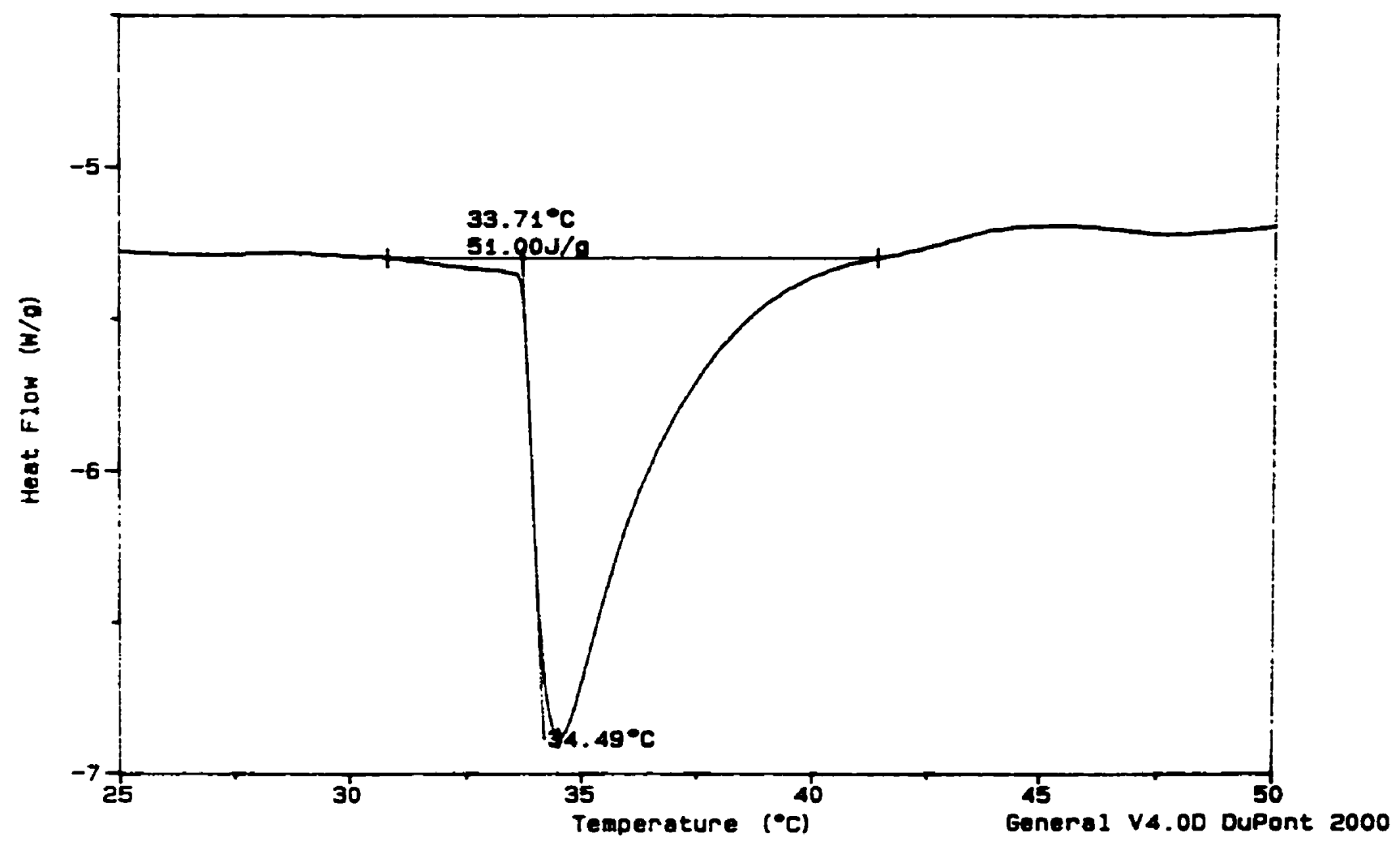


Sample: 2 c0-9010 7.00WTr IN H2O s1ze: $1.4140 \mathrm{mg}$

F118: A: $\operatorname{co2} .005$

Method: $\Omega$

Operator: U

Comment: $5.00 \mathrm{C} / \mathrm{MIN}$ TO $100 \mathrm{C}$

Run Date: 26-Aug-97 11: 44

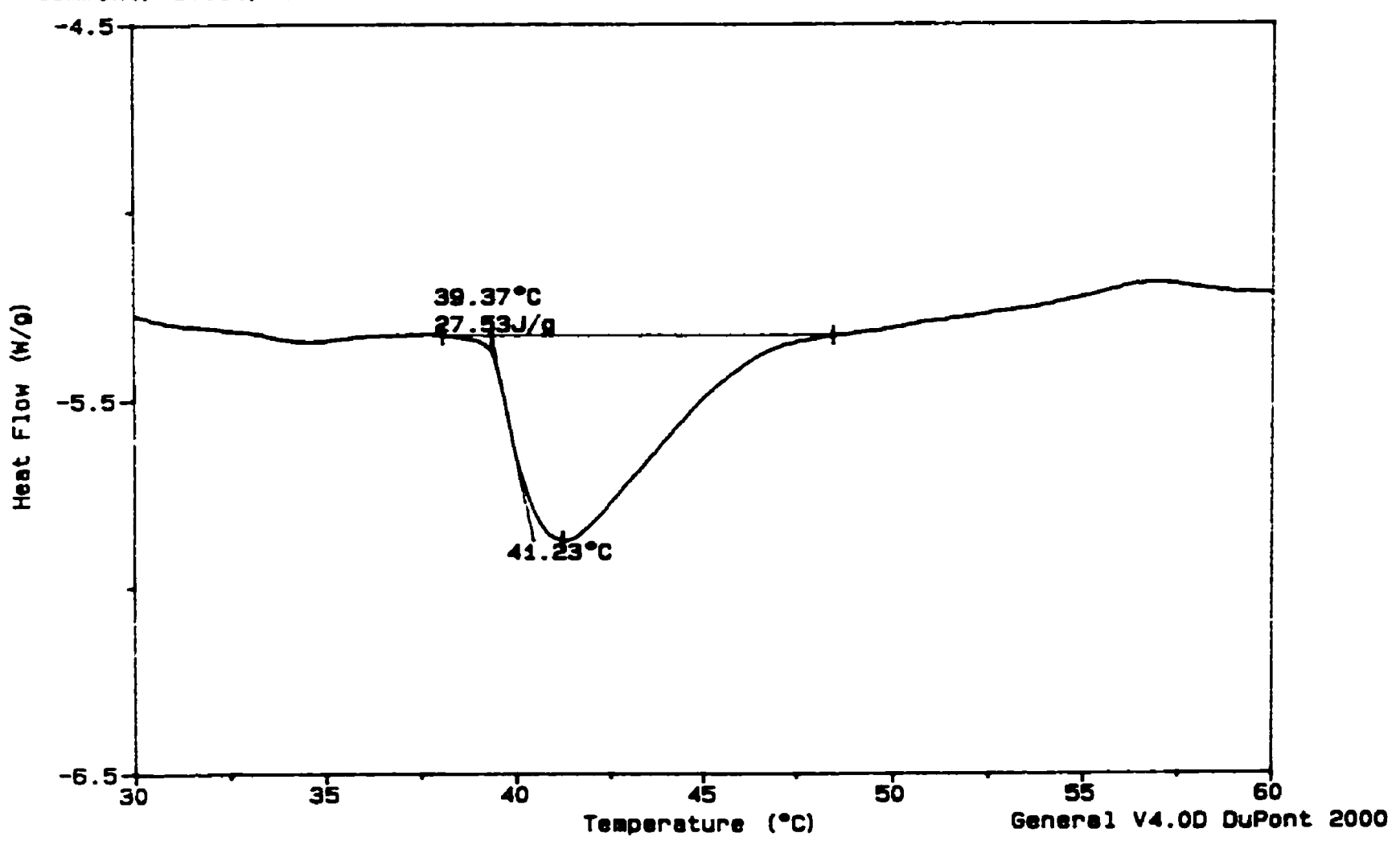




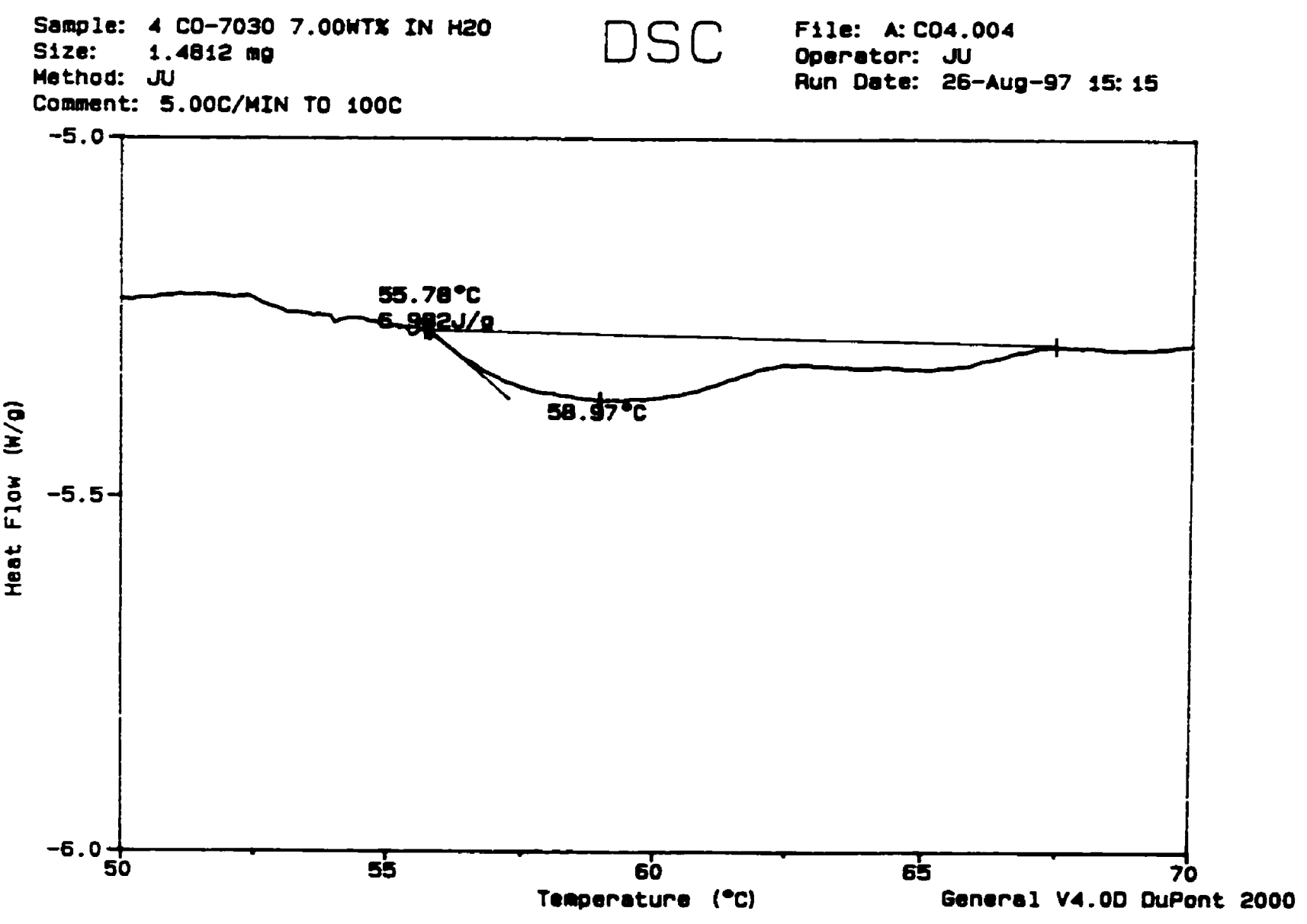




\section{Appendix 4.3 Calculation of the Mean Sequence Lengths}

"The mean sequence lengths" is a loose term for "the mean lengths of the closed monomer sequences", which is used to characterize the monomer sequence distribution and its deviation from the Bernoullian distribution. The objective of this calculation is to calculate the mean sequence lengths of the copolymers synthesied in Chapter 4.

According to Chiklis and Grasshoff [1], for the copolymerization of NIPAM and $A M$ in water at room temperature using ammonium persulfate and sodium metabisulfite as the redox initiator, the reactivity ratios are:
$r_{1}=$
0.5
(NIPAM)
$\mathbf{r}_{2}=$
1
(AM)

According to Pyun [2] , assuming the polymerization is a simple Markov process, the Mean Sequence Lengths

For NIPAM sequence distribution is

$$
\mu_{A}=1+r_{1} \frac{M_{1}}{M_{2}}
$$

For AM sequence distribution is

$$
\mu_{B}=1+r_{2} \frac{M_{2}}{M_{1}}
$$

where $M_{1}$ and $M_{2}$ are the monomer fractions in the feed for NIPAM and AM

\begin{tabular}{|c|c|c|c|c|}
\hline Samples & $\mathbf{M}_{\mathbf{1}}$ & $\mathbf{M}_{2}$ & $\mathbf{u}_{\mathbf{A}}$ & $u_{3}$ \\
\hline C0.9505 & 0.95 & 0.05 & 10.5 & 1.052632 \\
\hline CO-92.677.4 & 0.9268 & 0.0737 & 7.2877 & 1.079521 \\
\hline C0-9010 & 0.9 & 0.1 & 5.5 & 1.111111 \\
\hline CO-86.3/13.7 & 0.863 & 0.137 & 4.1496 & 1.158749 \\
\hline C0-75.9/24.1 & 0.759 & 0.241 & 2.5747 & 1.317523 \\
\hline Co.7030 & 0.7 & 0.3 & 2.1667 & 1.428571 \\
\hline CO-6040 & 0.6 & 0.4 & 1.75 & 1.666667 \\
\hline CO.5050 & 0.5 & 0.5 & 1.5 & 2 \\
\hline CO-4060 & 0.4 & 0.6 & 1.3333 & 2.5 \\
\hline CO-3070 & 0.3 & 0.7 & 1.2143 & 3.333333 \\
\hline CO-2080 & 0.2 & 0.7 & 1.1429 & 4.5 \\
\hline $\operatorname{co-1090}$ & 0.1 & 0.9 & 1.0556 & 10 \\
\hline
\end{tabular}
respectively.

Reference:

1. Chiklis, C. K. and Grasshoff, J. M., J. Polym. Sci.: Part A-2 , 8, 1617 (1970).

2. Pyun, C. W. . J. polym. Sci.: Part A-2 , 8, 1111 (1970). 


\title{
Chapter 5
}

\section{The Surface Tension Lowering Abilities of Poly(N-isopropylacrylamide) Microgels}

\begin{abstract}
Poly(N-isopropylacrylamide) (PNIPAM) microgels of different crosslinking degrees were synthesized through surfactant-free precipitation polymerization using redox initiation system and BA as the cross-linker at $70^{\circ} \mathrm{C}$. The microgels were characterized by measuring the ratios of their radii of gyration $\left(R_{\mathcal{g}}\right)$ to the theoretical hydrodynamic radii $\left(R_{h}\right) . R_{g}$ was obtained by static light scattering (SLS) through the dissymmetry method and $R_{h}$ was obtained by dynamic light scattering (DLS). $R_{g}$ varied from 70 to $87 \mathrm{~nm}$ and $R_{h}$ from 185 to $318 \mathrm{~nm}$ giving very small $R_{g} / R_{h}$ values ranging from 0.26 to 0.38 . It was found that cross-linked PNIPAM microgels were mono-dispersed in sizes by transmission electron microscopy (TEM). Environmental scanning electron microscopy (ESEM) found that the microgels actually adsorbed at the air/water interface in an orderly manner. The surface tension lowering abilities of the PNIPAM microgels before $\left(25^{\circ} \mathrm{C}\right)$ and after $\left(40^{\circ} \mathrm{C}\right)$ the volume phase transition
\end{abstract}


was verified through dynamic surface tension measurement using the axisymmetric drop shape analysis (ADSA-P) technology. The dynamic surface tension curves were fitted to Hua-Rosen Equation, giving a meso-equilibrium surface tension $\left(\gamma_{m}\right)$ range of 43.48 to $45.28 \mathrm{~mJ} / \mathrm{m}^{2}$ at $25^{\circ} \mathrm{C}$ and of 41.05 to 47.83 $\mathrm{mJ} / \mathrm{m}^{2}$ at $40^{\circ} \mathrm{C}$. It was found that both volume phase transition and crosslinking slowed down the surface tension lowering process, however, the mesoequilibrium surface tension was not very sensitive to either temperature or crosslinking.

Keyword: surface tension, microgels, poly $(N$-isopropylacrylamide $)$ 


\subsection{Introduction}

$N$-Isopropylacrylamide (NIPAM) can introduce thermal responsive behavior into polymers in aqueous media. Using $\mathrm{N}$-isopropylacrylamide (NIPAM) as the monomer or one of the comonomers, a class of stimuli sensitive "intelligent" hydrogels (macrogels or microgels) has been synthesized and investigated [1, 2, 3]. Crosslinked NIPAM hydrogels and its copolymer hydrogels have a mutual property, i.e. temperature sensitivity. Those hydrogels have a volume phase transition temperature $\left(T_{v}\right)$, which acts like an "on-off" switch controlling the thermal reversible properties of PNIPAM hydrogels. Pelton and Chibante first invented the monodispersed poly $(N$ isopropylacrylamide) (PNIPAM) microgels [1]. McPhee et al [4] found that the volume phase transition of $N, N^{\prime \prime}$-methylenebisacrylamide crosslinked microgels occurred between 30 and $34^{\circ} \mathrm{C}$ when $N, N$-methylenebisacrylamide composition varied from 0 to $15 \mathrm{wt} \%$ of NIPAM. Swelling of PNIPAM microgels was observed to decrease as temperature increase and their average diameter shrank by a factor of 2 , despite difference in crosslinker concentration [4]. The polymerwater interaction parameter $(\chi)$ of crosslinked PNIPAM was reported to increase from 0.524 to 0.971 when temperature was raised from 25 to $40^{\circ} \mathrm{C}$ [5]. It was found that the swelling and deswelling rates are inversely proportional to the square of the smallest dimension of a PNIPAM gel and the kinetics is controlled by diffusion-limited transport of polymeric components of the gel network in 
water $[6,7,8]$. Electrophoretic mobilities $[4,9,10]$, surfactant binding $[9,11,12$, $13,14,15]$ and turbidity of colloidal PNIPAM microgel suspensions were also temperature reversible [16].

The volume phase transition of crosslinked PNIPAM gels corresponds to the phase separation of linear PNIPAM homopolymer over its lower critical solution temperature (LCST) around $32{ }^{\circ} \mathrm{C}$. It is generally accepted that both the phase separation or volume phase transition are driven by the entropy gain through the release of water molecules from the cage-like structures around the isopropyl group [2, 5]. Hydrogen bonding and hydrophobic interactions between PNIPAM and water are the major interactions and the source of thermal reversible sensitivity [5]. Before volume phase transition, the swelling is attributed to the thermal mixing of molecules $[17,18,19,20]$. Through studying the temperature dependence of swelling of crosslinked poly( $N, N^{\prime}$-alkyl substituted acrylamides), Bae et al. concluded that before volume phase transition, the major hydration forces arise from hydrogen bonding interactions between amide groups and water molecules, but not from hydrophobic hydration, because the $\chi$ values were located around 0.5 , regardless of the size and configuration of the alkyl side groups [5]. The deswelling or shrinking upon heating through $T_{v}$ corresponds to the hydrophobic interaction induced association of segments [20].

Researches on NIPAM and its comonomer hydrogels have been mainly focused on the volume phase transition and temperature responsive behaviors [ 2 , 
$21,22,23,24,25,26,27,28,29,30,31,32]$, interactions with surfactants, synthesis of macrogels and microgels $[1,2,29,30,31,32,33,34,35,36,37,38$, $39,40,41]$ and potential applications. The studies on the potential applications include drug delivery $[42,43]$, biotechnology $[2,37,44]$, industry separation processes $[2,45]$, chromatography $[46,47]$ and optical devices [48].

We have been studying linear PNIPAM homopolymers $[49,50,51]$ adsorbed at the air/water interface through studying the surface tension behavior. It was found that PNIPAM could lower the surface tension of water down to a meso-equilibrium value of about $43 \mathrm{~mJ} / \mathrm{m}^{2}$ at $25{ }^{\circ} \mathrm{C}$ and about $40 \mathrm{~mJ} / \mathrm{m}^{2}$ at $40{ }^{\circ} \mathrm{C}$ [50]. It is known that the phase-separated PNIPAM can collapse from coils into globules and aggregate with each other to form colloidally stable particles $[2,49$, $50,52]$. Our previous work also revealed that poly( $N$-isopropylacrylamide-coacrylamide) copolymers could lower the surface tension of water both before and after the phase separation [53]. Surfactants and some polymers are well known to be able to lower the surface tension of water. While solid particles normally do not lower the surface tension of water, little is known about the surface tension lowering ability of microgels. Microgels are different from linear polymers in the respect that the backbone networks of microgels can not unwrap easily at the air/water interface due to the restrictions of chemical crosslinking, while linear polymers can. The fact that phase-separated colloidally stable PNIPAM particles 
can lower the surface tension of water has suggested the surface tension lowering ability of cross-linked PNIPAM microgels.

In this work, PNIPAM microgels of different crosslinking degrees were synthesized. Transmission electron microscopy (TEM) was used to see the shape and dispersity of the sizes of the microgels. Dynamic light scattering (DLS) and static light scattering (SLS) were used to characterize the dimension of the microgels. Environmental scanning electron microscopy (ESEM) was employed to see the microgels actually adsorbed at the air/water interface. The surface tension lowering ability of the PNIPAM microgels was verified through dynamic surface tension measurement using the same axisymmetric drop shape analysis (ADSA-P) technology as used in the previous works. 


\subsection{Experimental}

\subsubsection{Preparation of the Microgels}

The cross-linked poly $(N$-isopropylacrylamide) microgels used for the dynamic surface tension measurement were synthesized through surfactant-free precipitation polymerization. The monomer, $\mathrm{N}$-isopropylacrylamide (Kodak $\mathrm{Co}$.), was recrystallized twice from toluene/hexane. The cross-linker, $N, N^{*}-$ methylenebisacrylamide (BA) (Kodak Co.), and the initiator, ammonium persulfate (AP) (Aldrich), were used as received.

Similar to the synthesis method reported by Pelton et al [1], four microgel samples were prepared. Those microgels were different in the amount of crosslinker and the way it was added through choosing semi-batch or batch polymerization (Table 5. 1). Samples M-1, M-2 and M-3 were synthesized using semi-batch polymerization. In a semi-batch polymerization, $3.15 \mathrm{~g}$ NIPAM and half amount of BA required was dissolved in $380 \mathrm{ml}$ (semi-batch) or Milli $Q$ water and added into a 3-necked round bottom flask placed in a water bath. The flask was equipped with a reflux condenser, a mechanical stainless stirring rod with a Teflon paddle and a nitrogen bubbling needle. The solution was purged with $\mathrm{N}_{2}$ and heated to $70^{\circ} \mathrm{C}$. $0.1576 \mathrm{~g} \mathrm{AP}$ was dissolved in $10 \mathrm{ml}$ Milli $\mathrm{Q}$ water. The rest half amount of BA was also dissolved in $10 \mathrm{ml}$ Milli $\mathrm{Q}$ water. Both the solutions of AP and BA were purged with $\mathrm{N}_{2}$. The AP solution was added to the reaction flask in 
one shot, which was followed by $10 \mathrm{ml}$ of BA solution over the next 20 minutes if semi-batch polymerization was used. M-4 was synthesized through batch polymerization. In a batch polymerization, all the BA was dissolved with NIPAM in $390 \mathrm{ml}$ Milli $\mathrm{Q}$ water at the beginning and the rest is the same as in a semi-batch polymerization. The polymerizing mixture was well stirred at $500 \mathrm{rpm}$ A nitrogen blanket was maintained over the reaction mixture after initiating the polymerization. The reaction was complete 150 minutes after initiator (AP) addition [33].

The microgels were cleaned by 10 consecutive serum replacements. In this procedure a microgel suspension was centrifuged in a Beckman Model L7 Ultracentrifuge with a $60 \mathrm{Ti}$ rotor at a speed of $50,000 \mathrm{rpm}$ and $25 \mathrm{~min}$ for each time. The serum was decanted and replaced with water. The cleaned and uniformly dispersed microgel suspensions were stored at $4^{\circ} \mathrm{C}$ as stock suspensions. Weight analysis gave the microgel concentrations in wr\%, which are also shown in Table 5.1 . 
Table 5. 1 The crosslinker $N, N^{\prime}$-methylenebisacrylamide (BA) concentration, the methods used to add the crosslinker in the microgel synthesis and the stock suspension concentration. The feed NIPAM concentration was $0.07 \mathrm{M}$ and the ammonium persulfate $1.73 \times 10^{3} \mathrm{M}$. The polymerization temperature was $70^{\circ} \mathrm{C}$.

\begin{tabular}{|c|c|c|c|}
\hline Sample & $\begin{array}{c}\text { BA } \\
\text { concentration } \\
(w+\% *)\end{array}$ & $\begin{array}{c}\text { BA Addition } \\
\text { Method }\end{array}$ & $\begin{array}{c}\text { Mass conc. of stock } \\
\text { suspensions (wt\%) }\end{array}$ \\
\hline M-1 & 2 & Semi-batch & 1.49 \\
\hline M-2 & 6 & Semi-batch & 1.74 \\
\hline M-3 & 10 & Semi-batch & 2.60 \\
\hline$M-4$ & 10 & Batch & 1.76 \\
\hline
\end{tabular}

- weight percent of NIPAM in feed.

Separation of the microgels from water was done to see whether the microgel suspensions prepared above were clean of low molecular weight PNIPAM, which can lower the surface tension of water $[49,50]$. If the water samples collected in the following procedure did not have a surface tension lower than that of pure water, then the only surface tension lowering component in those dispersions was the microgels. To achieve the proper separation, Centreprep-PS (Amicon, Inc.) particle separator was used, which has a $0.2 \mu \mathrm{m}$ microporous filter membrane. Centreprep-PS is ideal for effectively removing particles from low molecular weight molecules in a solution. Prior to the dynamic surface tension measurement, $25 \mathrm{ml}$ of each of the samples listed in Table 5 . 1 was put into the outside tubes of 4 Centreprep-PS and spun at $5230 \mathrm{rpm}(\mathrm{g}$ force: $2900 \times \mathrm{g}$ ) in a Beckman CS-15R centrifuge for one hour. Then the water in the central tubes 
was collected for each sample. Each water sample was stationary in a sample beaker for 15.5 hours to ensure any possible polymer adsorption at the air/water interface. The surface tensions of those water samples were measured using a Fisher Model 215 Autotensiomat surface tension analyzer and they were all about $72 \mathrm{~mJ} / \mathrm{m}^{2}$, which is very close to $72.14 \mathrm{~mJ} / \mathrm{m}^{2}$, the surface tension of pure water at $25^{\circ} \mathrm{C}$ [54]. The $200 \mathrm{~nm}$ pore size of the particle separator is much larger than the radius of gyration of a linear polymer chain and should be able to allow any linear PNIPAM to pass through. From the previous work [50], it is known that at $25^{\circ} \mathrm{C}$, it took about two hours for $5 \mathrm{ppm}$ linear PNIPAM homopolymer to lower the surface tension of water to about $45 \mathrm{~mJ} / \mathrm{m}^{2}$ when its weight average molecular weight $\left(\bar{M}_{w}\right)$ was $5.47 \times 10^{5}(\mathrm{~L}-6 \mathrm{~B})$, and about one hour when its $\bar{M}_{w}$ was $1.31 \times 10^{4}(\mathrm{~L}-4)$. The surface tensions of those water samples indicated that within the time scale of about 15 hours and without the microgels, there was nothing else in the microgel suspensions could lower the surface tension of water.

\subsubsection{Microgel Characterizations}

\subsubsection{Transmission electron microscopy (TEM) Environmental Scanning Electron Measurement (ESEM)}

TEM images of all the four microgel samples were taken with a JEOL 1200EX TEM (JEOL Ld., Japan) operated at 80KV. For TEM, $10 \mu \mathrm{L}$ of each aqueous sample was pipetted onto 200 mesh Formvar coated $\mathrm{Cu}$ grid. Excess 
solution was blotted off with filter paper after $10 \mathrm{~min}$ and immediately added 5 $\mu \mathrm{L}$ of $2 \%$ phosphotungstic acid for $5 \mathrm{~min}$. Excess stain was gently blotted off with filter paper and the grid was left to air dry.

The PNIPAM microgels actually adsorbed at the air/water interface were probed in situ by ESEM Model 2020 (ElectroScan Corporation). A drop of the microgel suspension was placed on a stainless steel stub. The stub with the sample was then put into a Peltier Stage. The temperature was controlled at $1^{\circ} \mathrm{C}$ and the pressure at 5.9 Torr, which gave $100 \%$ relative humidity in the chamber.

\subsubsection{Dynamic Light Scattering Measurements}

The intensity-weighted mean hydrodynamic diameters of the four PNIPAM microgel samples were measured as a function of concentration and scattering angle at $25^{\circ} \mathrm{C}$ by dynamic light scattering or photon correlation spectroscopy. A Brookhaven Variable Angle QELS was used. It consisted of a Brookhaven Model BI-9000AT digital correlator, a BI-2000SM goniometer, and a Lexel (25 $\mathrm{mW}$ ) argon laser operating with an incident wavelength of $514 \mathrm{~nm}$ and a temperature-controlled water bath. The data were processed by the cumulant analysis and thus the Eff. Diam. $\left(D_{E}\right)$ was used. Although the microgels appeared to be spherical and nearly monodispersed in an aqueous medium according to the TEM and ESEM images, the sizes of those swollen microgels at $25^{\circ} \mathrm{C}$ were large and the Eff. Diam represented an angular dependent quantity 
[55]. To avoid the interaction between microgels and the dependence on angles at $25^{\circ} \mathrm{C}$, measurements at several concentration levels and at several incident angles were done. The concentration levels measured were 50 to $200 \mu \mathrm{g} / \mathrm{ml}$ for $\mathrm{M}-1$ and $M-2,4.04$ to $30 \mu \mathrm{g} / \mathrm{ml}$ for $M-3$ and 5 to $15 \mu \mathrm{g} / \mathrm{ml}$ for $M-4$. The incident angles were $25^{\circ}$ to $55^{\circ}$ for $\mathrm{M}-1$ and $\mathrm{M}-4,35^{\circ}$ to $90^{\circ}$ for $\mathrm{M}-2$, and $25^{\circ}$ to $45^{\circ}$ for $\mathrm{M}-3$. The results were extrapolated to the concentration of zero ppm and the angle of zero degree (see Appendix 5.1). The intercepts $\left(D_{h}\right)$ were theoretically well defined $[55,56,57]$ and are used to obtain the $R_{h}\left(=D_{h} / 2\right)$ of the microgels at 25 ${ }^{\circ} \mathrm{C}$.

After the volume phase transition, the microgels shrunk to about half their swollen sizes, measurements at $90^{\circ}$ incident angle of very dilute microgel suspensions (count rate about $50 \sim 100 \mathrm{kcnts} / \mathrm{s}$ ) were used to obtain the hydrodynamic radii at $40^{\circ} \mathrm{C}$.

\subsubsection{Static light scattering measurements and the dissymmetry method [58]}

The values of the radius of gyration $\left(R_{\varepsilon}\right)$ of the four microgel samples were obtained through the classical static light scattering experiment and the measurement of the dissymmetry of the light scattered by those microgels. This method views the microgels as core-shell structured spheres [59]. By theory, if a particle's core diameter $D$ is greater than $\frac{\lambda_{0}}{20 n}$, where $\lambda_{0}$ is the wavelength in 
vacuum and $n$ the refractive index of the medium, the intensity of the scattered light by such large particles is dissymmetric with respect to the incident direction of $90^{\circ}$. The dissymmetry ratio, $Z$, is defined as:

$$
Z=\frac{I_{\theta}}{I_{(180-\theta)}}
$$

\section{Equation 5.1}

where $I_{\theta}$ and $I_{(180-\theta)}$ is the total intensity of the light scattered by the particles and measured at angle $\theta\left(\theta<90^{\circ}\right)$ and $180^{\circ}-\theta$. The intensity ratio equals to the ratio of the scattering function $P(\theta) / P(180-\theta)$. Therefore

$$
Z=\frac{I_{\theta}}{I_{(180-\theta)}}=\frac{P(\theta)}{P(180-\theta)}
$$

\section{Equation 5.2}

The core size of monodispersed large particle with certain known shapes can be determined through the measurement of $Z$. For homogeneous spheres of core diameter $D$, it has been deducted [58] that

$$
P(\theta)=\left[\left(\frac{3}{x^{3}}\right)(\sin x-x \cos x)\right]^{2}
$$

Equation 5.3

where

$$
x=2 \pi\left(\frac{D n}{\lambda_{0}}\right) \sin \left(\frac{\theta}{2}\right)
$$

Equation 5.4

The pair of angles $45^{\circ}$ and $135^{\circ}$ has been used predominantly in practice, partially because of tradition and partially because of rationality that the scattering 
intensity at these angles can be measured with relative accuracy. Once $D$ is obtained, the radius of gyration $\mathbf{R}_{\mathrm{g}}$ can be calculated [57] using

$$
R_{8}^{2}=\frac{3}{5}\left(\frac{D}{2}\right)^{2}
$$

\section{Equation 5.5}

The total intensity as a function of microgel concentration $(C)$ was measured at scattering angle $45^{\circ}, 90^{\circ}$ and $135^{\circ}$ using the same equipment described above, setting the duration to $1 \mathrm{~s}$ and the sample time to $1 \mu \mathrm{s}$, as instructed in the equipment manual [55]. The concentration ranges were the same as in DLS measurements. Background intensity from dark counts and scattering from water was subtracted. The dissymmetry ratios were calculated from $\frac{I_{45}}{I_{135}}$. Z versus $\mathrm{C}$ had good linearity and the data were extrapolated to zero concentration. $\boldsymbol{R}_{\boldsymbol{g}}$ was calculated using Equations 5.2 to 5.5 (see Appendix 5.2).

\subsubsection{Surface Tension Measurements}

ADSA-P is a technique that can be used to determine surface tensions from the shape of the axisymmetric menisci of a pendent drop. The strategy employed is to fit the shape of a drop to the theoretical drop profile according to the Laplace equation, using surface tension as one of the adjustable parameters. The best fit gives the correct or operative surface tension. The error limits of these measurements are on the order of $0.1 \mathrm{~mJ} / \mathrm{m}^{2}$ or less. The ADSA- analysis required accurate density measurements, which were measured as functions of 
temperature and microgel concentration with an AP PAAR DMA45 MC 1296 densitometer. Some of the density results are summarized in Error! Reference source not found.. Pendent drops were formed on the tip of a Teflon capillary with an outside diameter of 0.1 in. and inside diameter of 0.076 in. (Chromatographic Specialties Inc.). Fluid flow to the capillary was controlled with a Hamilton Gastight syringe (Chromatographic Specialties Inc.). The pendent drop was enclosed in a sealed quartz cuvette (Hellma 330984, $10 \times 10 \times 30 \mathrm{~mm}^{3}$ ), which was mounted in a Model 100-07 environmental chamber (Rame Hart Inc.). The temperature control was $\pm 0.1{ }^{\circ} \mathrm{C}$. Further details of the apparatus and methodology can be found elsewhere $[60,61]$. The Milli $Q$ water used to prepare the microgel suspensions had a surface tension of $72.20 \mathrm{~mJ} / \mathrm{m}^{2}$ at $25.0^{\circ} \mathrm{C}$, which is in good agreement with $72.14 \mathrm{~mJ} / \mathrm{m}^{2}$ given by Jasper [54]. 
Table 5. 2 Density of the aqueous microgel suspensions used in the surface tension measurement.

\begin{tabular}{|c|c|c|c|}
\hline Sample & $\mathbf{T}\left({ }^{\circ} \mathrm{C}\right)$ & $\mathbf{C}(\boldsymbol{m})$ & Density $\left(\mathbf{k g} / \mathbf{m}^{3}\right)$ \\
\hline M-1 & 25 & 0.05 & 0.9967 \\
& & 1 & 0.9980 \\
& 40 & 0.05 & 0.9922 \\
& & 1 & 0.9929 \\
\hline M-2 & 25 & 0.05 & 0.9967 \\
& & 1 & 0.9980 \\
& 40 & 1 & 0.9929 \\
\hline M-3 & 25 & 0.05 & 0.9970 \\
& & 1 & 0.9979 \\
& 40 & 1 & 0.9929 \\
\hline M-4 & 25 & 0.05 & 0.9922 \\
& & 1 & 0.9936 \\
& 40 & 1 & 0.9936 \\
\hline
\end{tabular}




\subsection{Results}

Microgels $M-1, M-2$ and $M-3$ were synthesized through semi-batch polymerization and M-4 through batch polymerization. In a semi-batch polymerization, half of the BA was mixed with NIPAM before the polymerization was initiated and the remainder was added dropwise within $20 \mathrm{~min}$ of the initiation. Whereas, in the batch polymerization, all the amount of BA was mixed with NIPAM before initiation. The difference of the addition of the crosslinker in these two kinds of polymerization resulted in variation of the distribution of BA, and therefore, the structures and properties of the microgels synthesized thereby (more details in discussion section).

\subsubsection{Microgel Characterizations}

The TEM micrographs were taken at 10,000X and shown in Figure 5. 1 . TEM shows the sizes and their distributions of dried microgel spheres. The images in Figure 5. 1 are probably only discs collapsed from microgel spheres due to water evaporating. The approximate diameters of the dried microgel were: $350 \sim 400 \mathrm{~nm}$ for $\mathrm{M}-1,450 \sim 600 \mathrm{~nm}$ for $\mathrm{M}-2,350 \sim 400 \mathrm{~nm}$ for $\mathrm{M}-3$ and $350 \sim$ $400 \mathrm{~nm}$ for M-4. Those TEM micrographs indicates that all the four kinds of microgels were nearly monodispersed and uniform in size. There was bridging between some of the dried microgels. The dried $M-3$ and M-4 appeared to be better defined in shape than $M-1$ and $M-2$. 
Figure 5.1A TEM images of the microgels. The bar in each micrograph indicate 1 micrometer in length 


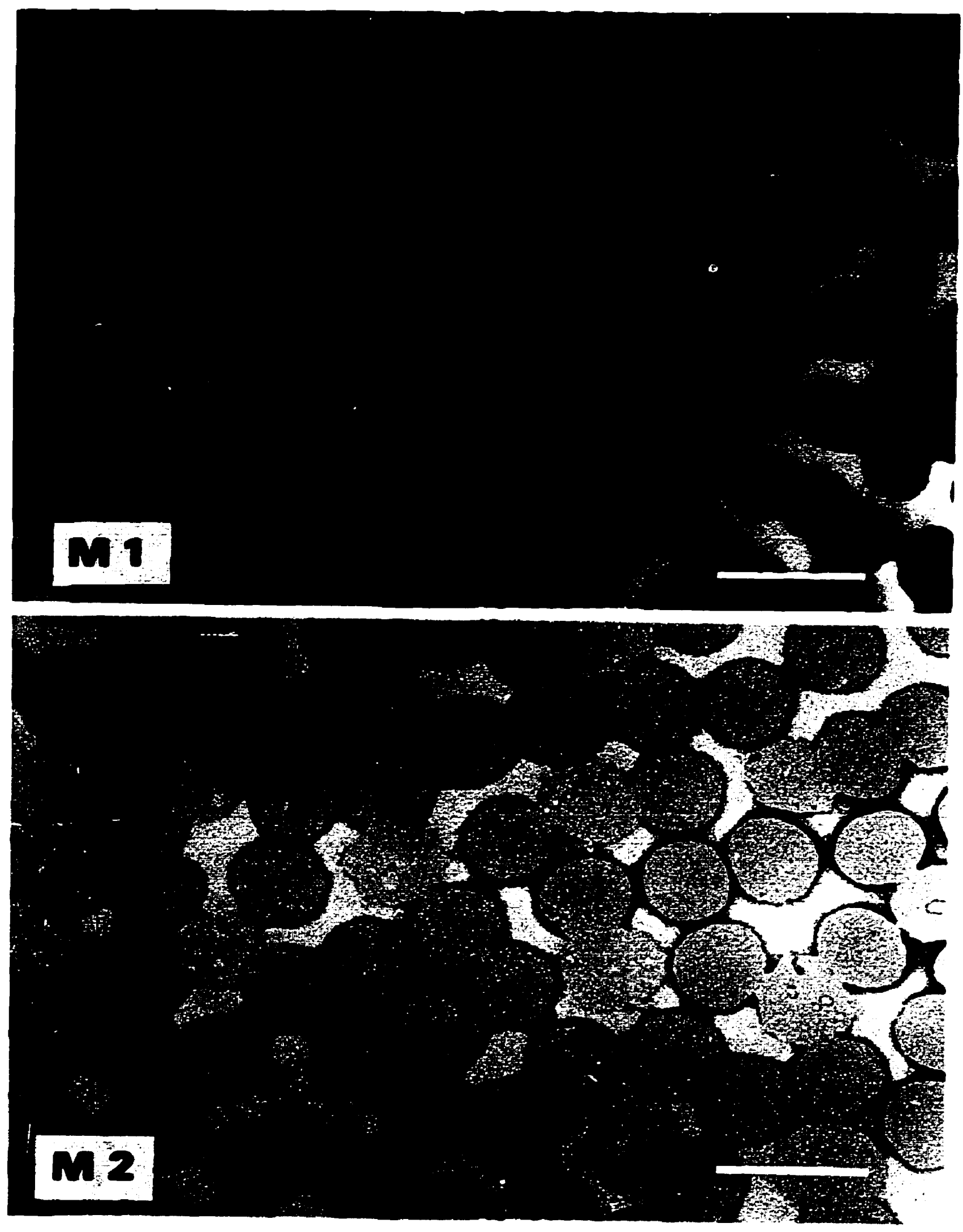


Figure 5. 1B TEM images of the microgels. The bar in each micrograph indicate 1 micrometer in length. 


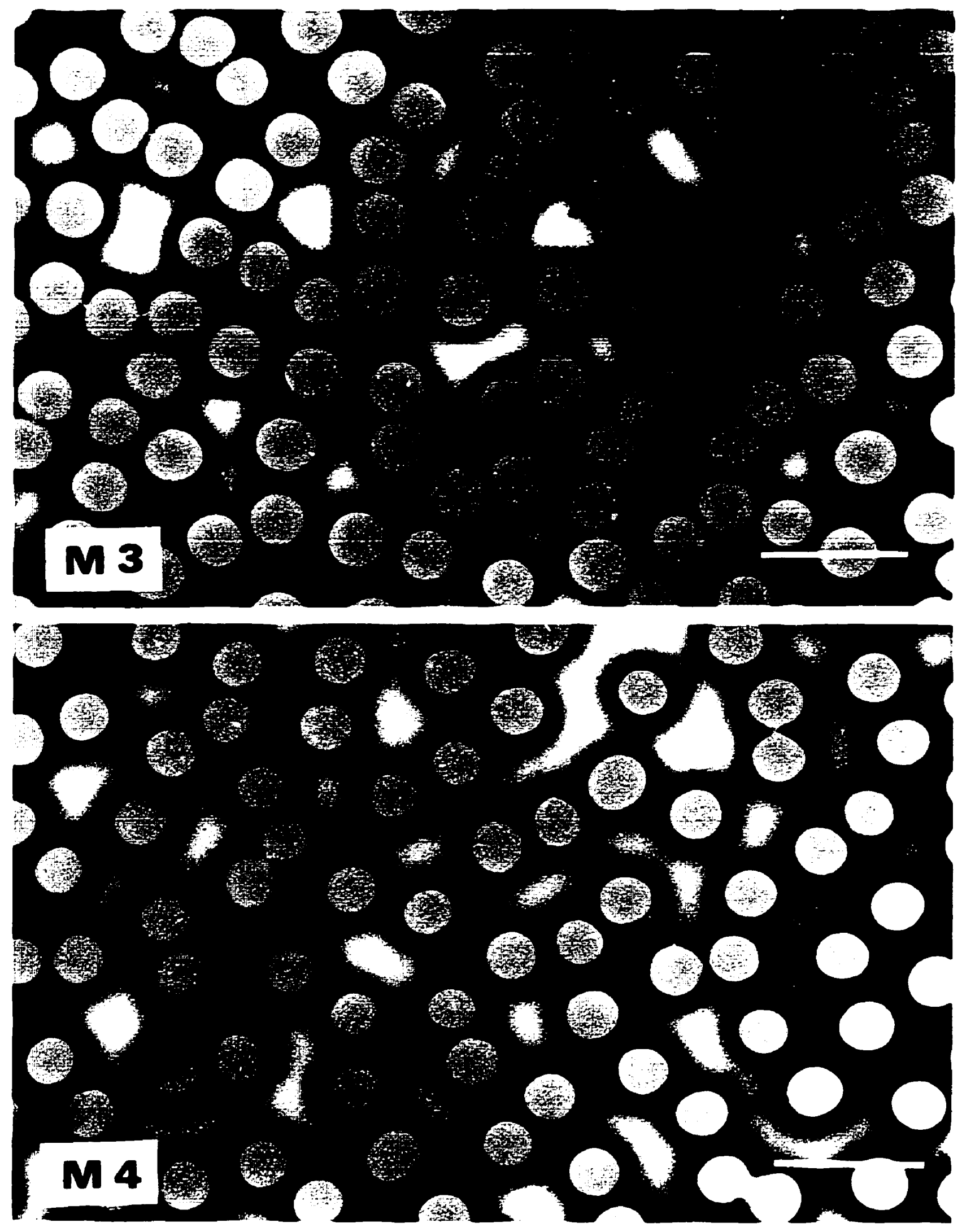


Figure 5. 2 The image of M-3 microgels adsorbed at the air/water interface probed in situ by environmental scanning electron microscopy (ESEM). The temperature was controlled at $1{ }^{\circ} \mathrm{C}$ and the pressure at 5.9 Torr, which gave $100 \%$ relative humidity in the chamber 


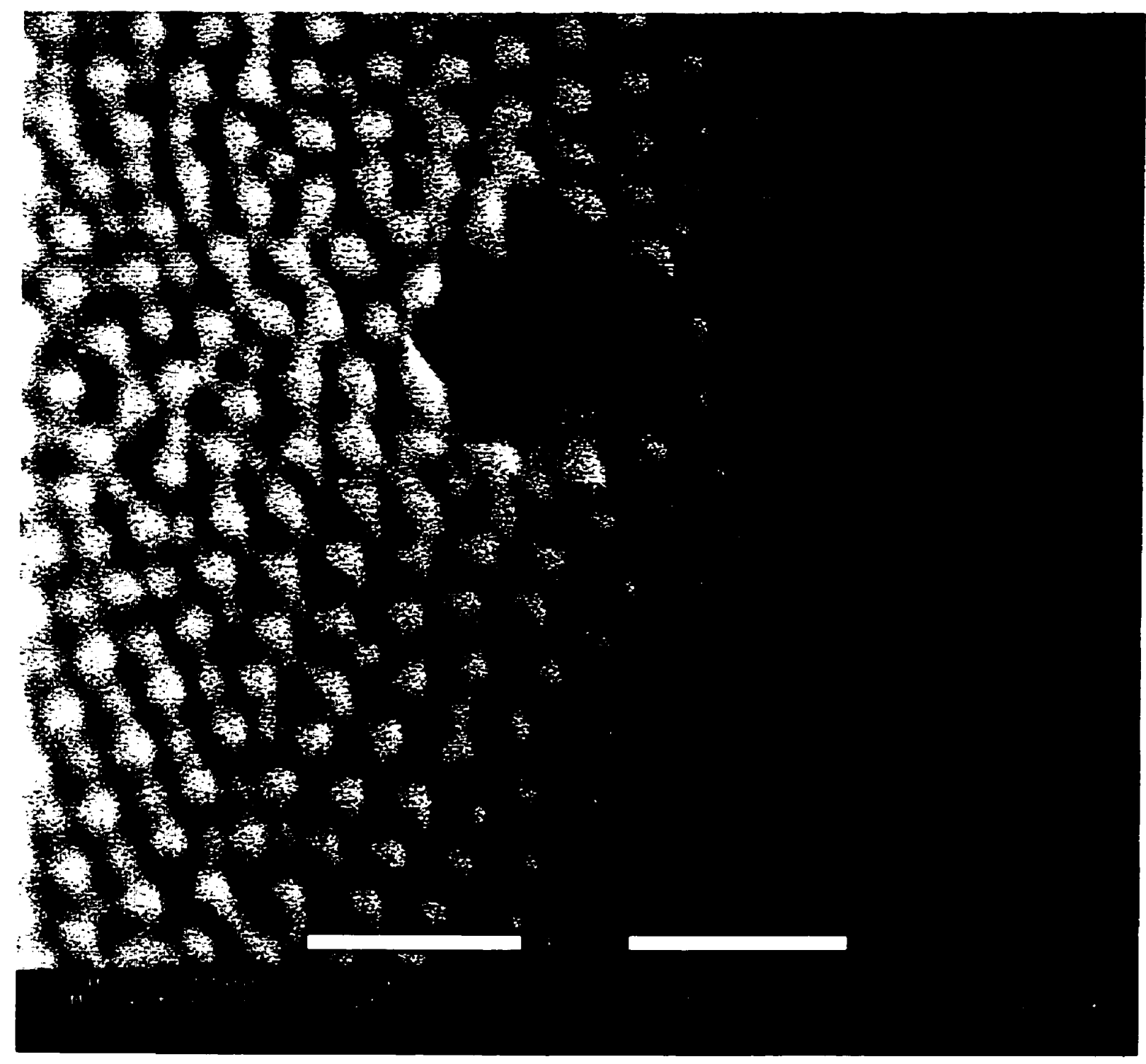


The images shown in Figure 5. 2 is of M-3 microgels adsorbed at the air/water interface, which were obtained by ESEM. Microgels with less crosslinker (M-1 and M-2) gave obscure images and the effort to get better images was abandoned. This picture shows that PNIPAM microgel spheres adsorbed at the air/water interface in an ordered manner. This was the first time microgels in submicron size adsorbed at air/water interface has ever been seen and reported.

The intensity-weighted mean hydrodynamic diameters of the four PNIPAM microgel samples were measured as a function of concentration and scattering angle at $25^{\circ} \mathrm{C}$ by dynamic light scattering. The results were extrapolated to zero concentration and zero angle to avoid interaction between particles and angular effect. The intercepts $\left(D_{h}\right)$ are used to obtain the theoretical hydrodynamic radii, $R_{h}\left(=D_{h} / 2\right)$, of the microgels at $25^{\circ} \mathrm{C}$. The values of the radii of gyration $\left(R_{\varepsilon}\right)$ of the four microgel samples were obtained through the classical static light scattering experiment at $25^{\circ} \mathrm{C}$ and the dissymmetry method. Both $R_{h}$ and $R_{g}$ are summarized in Table 5. 3. At $25^{\circ} \mathrm{C}$ in water, $R_{h}$ was between $185 \sim 318 \mathrm{~nm}$ and $R_{g}$ between $70 \sim 87 \mathrm{~nm}$. The increase order of $R_{h}$ of microgels is $\mathrm{M}-1<\mathrm{M}-2<\mathrm{M}-4<\mathrm{M}-3$. The increase order of $\boldsymbol{R}_{\boldsymbol{g}}$ of microgels is, however, M$1<\mathrm{M}-2<\mathrm{M}-3<\mathrm{M}-4$. Accordingly, the values of $\boldsymbol{R}_{g} / \boldsymbol{R}_{\mathfrak{h}}$ changed between $0.26 \sim$ 0.38 in the order of $M-1>M-4>M-2>M-3$. The intensity-weighted hydrodynamic radii from the incident direction of $90^{\circ}$ at both $25^{\circ} \mathrm{C}\left(R_{h, 2 s C}\right)$ and $40^{\circ} \mathrm{C}\left(R_{h, 40 C}\right)$ for dilute suspensions were also measured and listed in Table 5. 3. 
Table 5. 3 The values of the theoretical hydrodynamic radius $\left(R_{h}\right)$, the radius of gyration $\left(R_{z}\right)$ and $R_{z} / R_{A}$ for the microgels in an aqueous medium at 25 ${ }^{\circ} \mathrm{C}$, the intensity weighted hydrodynamic radius at $90^{\circ}$ for dilute dispersions at $25^{\circ} \mathrm{C}\left(R_{\mathrm{h}, 25 \mathrm{C}}\right)$ and $40^{\circ} \mathrm{C}\left(R_{h, 10 \mathrm{C}}\right)$, and the swelling ratio $\phi_{\text {swelling }}$.

\begin{tabular}{|c|c|c|c|c|c|c|}
\hline Sample & $R_{n}(\mathrm{~nm})$ & $\overline{R_{8}(n m)}$ & $\mathbf{R}_{\boldsymbol{d}} \mathbf{R}_{\mathrm{m}}$ & $R_{m, 25 c}(\mathrm{~nm})$ & $\mathbf{R}_{\mathrm{h}, \sec (\mathrm{nm})}$ & stomen \\
\hline M-1 & 185 & 70 & 0.38 & $271 \pm 11$ & $125 \pm 2$ & 2.2 \\
\hline$\overline{\mathbf{M}-2}$ & 269 & 75 & 0.28 & $297 \pm 2$ & $160 \pm 12$ & 1.9 \\
\hline$\overline{\mathbf{M}-3}$ & 318 & $\overline{83}$ & 0.26 & $325 \pm 7$ & $172 \pm 10$ & 1.9 \\
\hline$\overline{M-4}$ & 297 & 87 & 0.29 & $289 \pm 3$ & $147 \pm 5$ & 2.0 \\
\hline
\end{tabular}

\subsubsection{Dynamic Surface Tension Measurement}

The dynamic surface tension of the four microgel samples is displayed in Figure 5. 3 to Figure 5. 5. At a low bulk concentration level of 0.05 wt\%, only M-1 could lower the surface tension of water both before and after the volume phase transition within the time scale of experiment, as shown in Figure 5. 3. Microgels of higher crosslinking degrees failed to lower the surface tension of water within the same time scale. At such a dilute concentration level, loosely cross-linked M-1 lowered the surface tension of water down to about $45 \mathrm{~mJ} / \mathrm{m}^{2}$ at $25^{\circ} \mathrm{C}$ within an hour and to $46.5 \mathrm{~mJ} / \mathrm{m}^{2}$ at $40^{\circ} \mathrm{C}$ within two hours. The surface tension of pure water was $72.14 \mathrm{~mJ} / \mathrm{m}^{2}$ at $25^{\circ} \mathrm{C}$ and $69.92 \mathrm{~mJ} / \mathrm{m}^{2}$ at $40{ }^{\circ} \mathrm{C}$ [54]. Although the initial surface tension at $40^{\circ} \mathrm{C}$ was lower than that at $25^{\circ} \mathrm{C}$ because of the temperature effect, the meso-equilibrium surface tension had values around $45 \mathrm{~mJ} / \mathrm{m}^{2}$ at the both temperatures. All the four kinds of microgels lowered the 
surface tension of water significantly when the bulk concentration was raised to 1 $w t \%$ at both 25 and $40^{\circ} \mathrm{C}$. The dynamic surface tension curves of 1 wt\% linear PNIPAM homopolymer (L-6B) were plotted with those of the microgels in Figure 5. $4\left(25^{\circ} \mathrm{C}\right)$ and Figure 5.5 $\left(40^{\circ} \mathrm{C}\right)$.

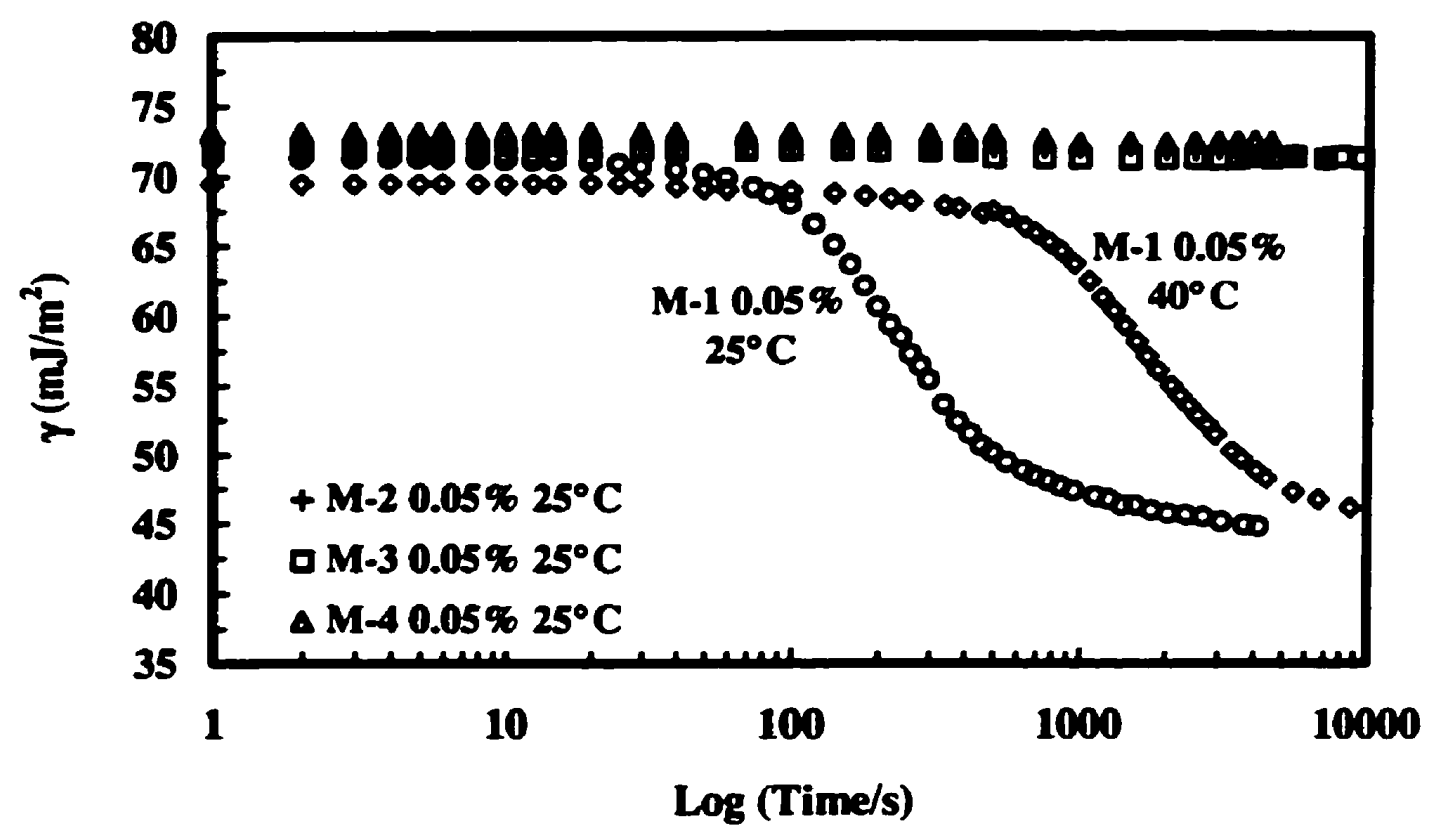

Figure 5.3 The experimental dynamic surface tensions of the four microgel samples at bulk concentration level of $0.05 w t \%$. 


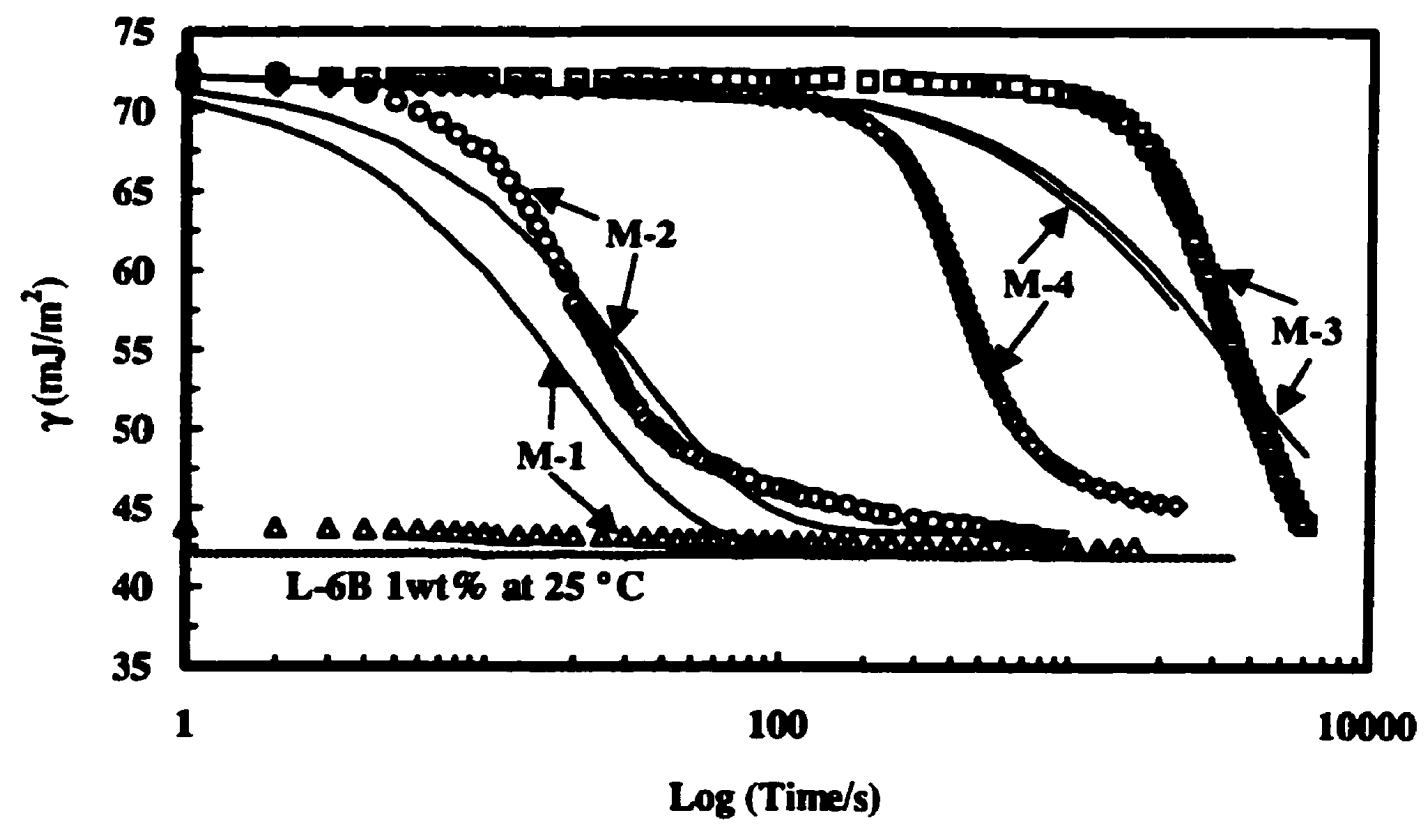

Figure 5.4 The experimental dynamic surface tensions of the four microgel samples at $25^{\circ} \mathrm{C}$ at bulk concentration level of $1 \mathrm{wt} \%$ and corresponding model prediction (solid lines) with $k_{1}=1.63 \times 10^{-4} \mathrm{~s}^{-1}$. 


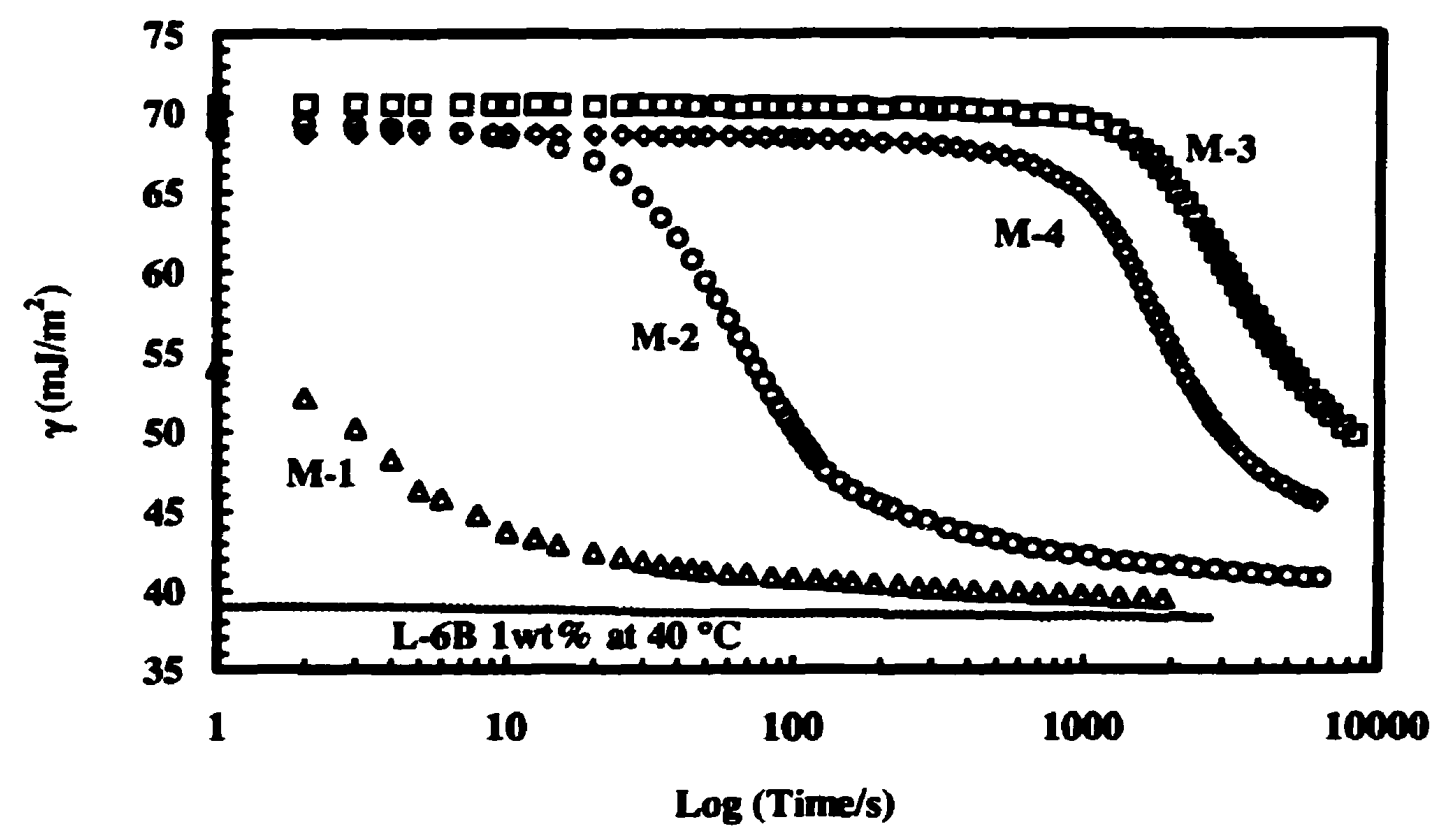

Figure 5.5 The experimental dynamic surface tensions of the four microgel samples at $40^{\circ} \mathrm{C}$ at bulk concentration level of $1 \mathrm{wt} \%$.

The following Hua and Rosen equation has been used to describe the dynamic surface tension of a surfactant solution $[62,63]$ :

$$
\log \frac{\gamma_{0}-\gamma(t)}{\gamma(t)-\gamma_{*}}=n \log \frac{t}{t^{*}}
$$

where $\gamma(t)$ is the dynamic surface tension, $\gamma_{0}$ is the surface tension at the instant the drop is formed which is assumed to equal the surface tension of pure water, $n$ is an empirical dimensionless constant, $\gamma_{m}$ is the meso-equilibrium surface tension, and $i$ is the time at which $\gamma(t)$ is half way between $\gamma_{0}$ and $\gamma_{m}$, i.e. $\frac{\gamma_{0}-\gamma_{m}}{2} . i$ can be used to estimate how fast a surface tension lowering process 
is. The Hua-Rosen parameters describing the dynamic surface tensions of the four microgel samples are listed in Table 5. 4. The surface tension lowering processes of M-1 were the fastest of all the four microgel samples at both 25 and $40^{\circ} \mathrm{C}$, although slower than the corresponding 1 wt\% linear homopolymer (L-6B) solution. The minimum dynamic surface tension of $1 \mathrm{wt} \% \mathrm{M}-1$ was $42.47 \mathrm{~mJ} / \mathrm{m}^{2}$ at $25{ }^{\circ} \mathrm{C}$ and $39.45 \mathrm{~mJ} / \mathrm{m}^{2}$ at $40^{\circ} \mathrm{C}$. Hua-Rosen parameters for $1 \mathrm{wt} \% \mathrm{M}-1$ dynamic surface tension curves were not listed because the surface tension lowering process was too fast and no reliable curve fitting could be obtained.

The meso-equilibrium surface tension varied between 43.5 to $45.28 \mathrm{~mJ} / \mathrm{m}^{2}$ at $25^{\circ} \mathrm{C}$ and between 41.05 and $47.83 \mathrm{~mJ} / \mathrm{m}^{2}$ at $40^{\circ} \mathrm{C}$. It appears that $\gamma_{m}$ is not very sensitive either to temperature or to crosslinking degree, even though phase separation did make the variation broader. The volume phase transition did increase $t^{*}$ for all the microgel samples. The most significant change brought through increasing the cross-linking degree was the change of $i$. Figure 5.6 shows $t^{*}$ as a function of BA concentration (wt\% of NIPAM). $t^{*}$ increased in the order of L-6B >M-1 >M-2 >M-4 >M-3 at both 25 and $40^{\circ} \mathrm{C}$, which was consistent with the $R_{h}$ order. Judged from how fast the dynamic surface tension reached $\frac{\gamma_{s}-\gamma_{m}}{2}$, it was clear that the surface tension lowering abilities were in the order of L-6B > M-1 > M-2 >M-4 > M-3, before or afier volume phase transition. The surface tension lowering ability order drawn from Table 5. 4, 
Figure 5. 3, Figure 5. 4 and Figure 5. 5 suggests that both cross-linking of PNIPAM chains and polymer chain aggregation due to dehydration at the volume phase transition slow down the surface tension lowering process. The polymer chain aggregation was caused by deswelling of the microgels at volume phase transition between 30 and $34^{\circ} \mathrm{C}$ [4].

Table 5. 4 Hua-Rosen parameters $[62,63]$ describing the dynamic surface tensions of the four microgel samples and the meso-equilibrium IP group concentration at the air/water interface.

\begin{tabular}{|c|c|c|c|c|c|c|c|c|c|}
\hline Somple & $\begin{array}{c}\mathrm{T} \\
\left({ }^{\circ} \mathrm{C}\right)\end{array}$ & $\begin{array}{c}C_{0} \\
(w \%)\end{array}$ & $n$ & (s) & $\underset{\left(m / m^{2}\right)}{x}$ & $\boldsymbol{R}^{2}$ & $\begin{array}{c}\Gamma i r m \\
\left(m g / m^{2}\right)\end{array}$ & (s) & $R_{m}^{2}$ \\
\hline M-1 & $\begin{array}{l}25 \\
40\end{array}$ & $\begin{array}{c}0.05 \\
1 \\
0.05 \\
1\end{array}$ & $\begin{array}{l}1.7791 \\
1.8647\end{array}$ & $\begin{array}{c}234.4 \\
-0 \\
1687.5 \\
-0\end{array}$ & $\begin{array}{c}44.96 \\
42.47^{* *} \\
44.88 \\
39.45^{* *}\end{array}$ & $\begin{array}{l}0.9961 \\
0.9992\end{array}$ & $\begin{array}{l}0.27 \\
0.31\end{array}$ & 13 & 0.9927 \\
\hline$\overline{M-2}$ & $\begin{array}{l}25 \\
40\end{array}$ & $\begin{array}{l}1 \\
1\end{array}$ & $\begin{array}{l}1.5526 \\
1.3665\end{array}$ & $\begin{array}{l}20.8 \\
64.6\end{array}$ & $\begin{array}{l}43.48 \\
41.05\end{array}$ & $\begin{array}{l}0.9949 \\
0.9904\end{array}$ & 0.28 & 21 & 0.9979 \\
\hline$\overline{M-3}$ & $\begin{array}{l}25 \\
40\end{array}$ & $\begin{array}{l}1 \\
1\end{array}$ & $\begin{array}{l}3.3978 \\
2.5842\end{array}$ & $\begin{array}{l}3024.1 \\
3407.5\end{array}$ & $\begin{array}{c}43.5 \\
47.83\end{array}$ & $\begin{array}{l}0.9985 \\
0.9984\end{array}$ & 0.28 & 2281 & 0.9947 \\
\hline$\overline{\mathbf{M}-4}$ & $\begin{array}{l}25 \\
40\end{array}$ & $\begin{array}{l}1 \\
1\end{array}$ & $\begin{array}{l}3.1139 \\
2.3321\end{array}$ & $\begin{array}{l}424.4 \\
1745.6\end{array}$ & $\begin{array}{l}45.28 \\
44.27\end{array}$ & $\begin{array}{l}0.9992 \\
0.9971\end{array}$ & 0.27 & 1941 & 0.9926 \\
\hline
\end{tabular}

Note:

$R^{2}=1-\frac{\sum_{0}^{k}\left(\gamma_{i}-\gamma_{i, f}\right)^{2}}{\sum_{0}^{k}\left(\gamma_{i}\right)^{2}-\frac{\left(\sum_{0}^{k} \gamma_{i}\right)^{2}}{k+1}}$, where $\gamma_{i}$ is the experimentally measured surface tension and $\gamma_{i f}$ is the fined value.

" The minimum dynamic surface tension. 


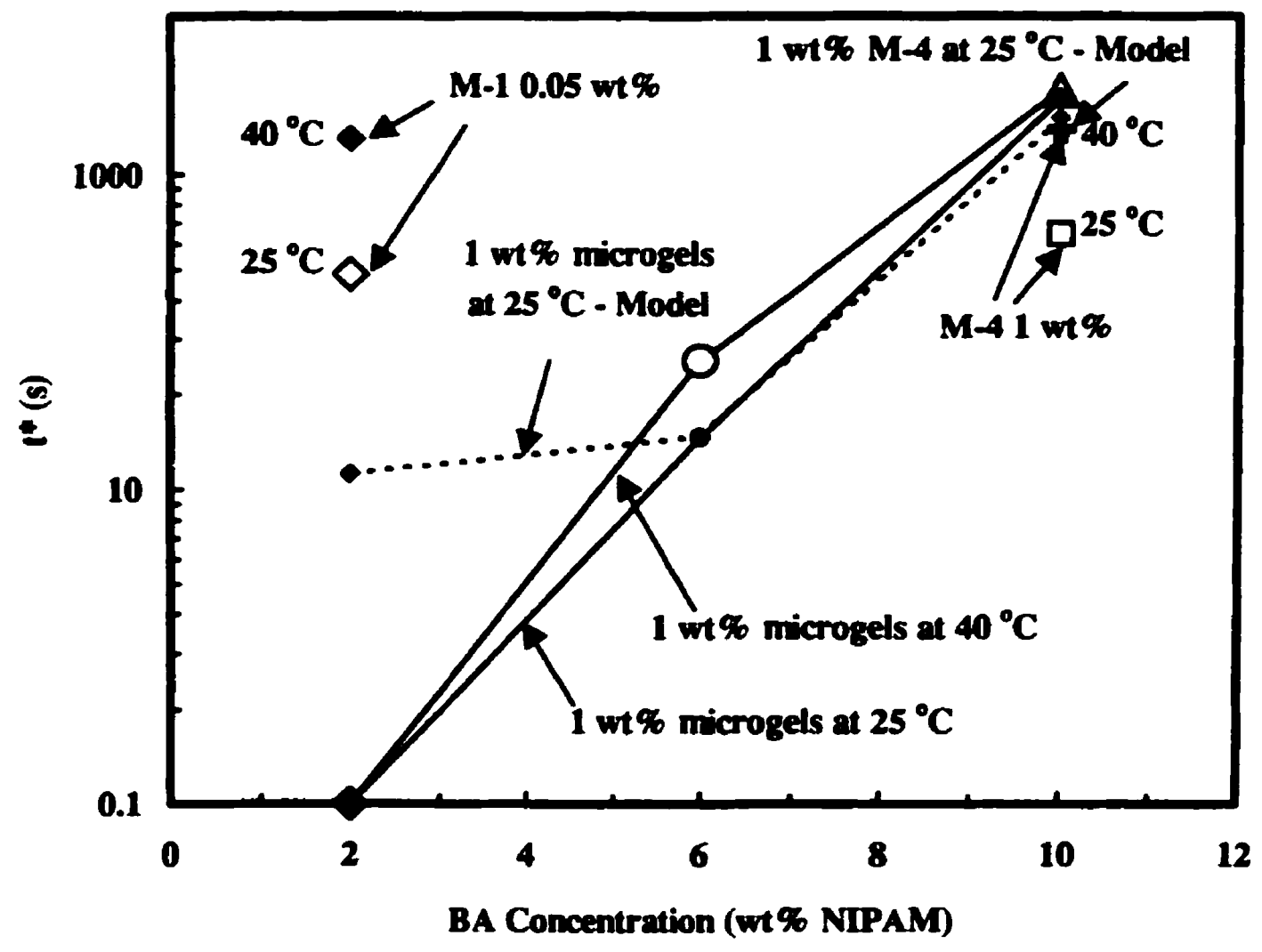

Figure 5.6 $t^{*}$ as a function of crosslinker (BA) concentration ( $w t \%$ of NIPAM). 


\subsection{Discussion}

The value of $R_{g} / R_{h}$ provides information about the microgel structure. Theoretically, this ratio is 1.73 for a random coil in a $\theta$ solvent and 0.775 for a homogeneous hard sphere [64]. At $25^{\circ} \mathrm{C}$, the values of this ratio for the four kinds of PNIPAM microgels are far below the homogeneous "hard sphere" value, which is no surprise. It has been repeatedly reported that $R_{g} / R_{h}$ values for microgels lie well below this "hard sphere" value. The value of $R_{g} / R_{h}$ was 0.548 1.853 for polyvinylacetate (Schmidt et al) [64], $0.49 \sim 0.58$ for polybutylmethacrylate (Kunz et al) [65]. For polymethylmethacrylate, $R_{g} / R_{h}$ was reported to be 0.527 by Kuns and Burchard [66], $0.47 \sim 0.72$ by Nieuwenhuis et al [67] and $0.5485 \sim 0.6605$ by Wolfe and Scopazz [68]. The fact that the $R_{g} / R_{h}$ values for microgels are well below the "hard sphere" value has been interpreted as caused by the dangling chains attached to a not so well defined sphere surface, i.e. rough surface. Those dangling chains produce a much slower decay of the segment density than in the case of a hard sphere with smooth surface and a larger hydrodynamic radius, smaller $R_{g} / R_{h}[56,68]$. It was reported that phase-separated stable single PNIPAM chain had a $R_{g} / R_{h}$ ratio as low as of 0.62 [69], but a lot higher than the values for PNIPAM microgels in this work.

In previous works $[49,50]$, it was found that phase-separated linear PNIPAM homopolymers could lower the surface tension of water at $40{ }^{\circ} \mathrm{C}$ although with a slower kinetics compared with the surface tension lowering 
process before phase separation. The surface tension lowering ability is influenced by the mobility of PNIPAM chains, which can be changed either through crosslinking by BA and polymer chain aggregation due to phase separation of linear PNIPAM or volume phase transition of gels.

The microgels having higher crosslinking degree are less flexible or deformable, and the polymer chains between crosslinks in the gel networks are less mobile. If assume that quantitative reaction of both NIPAM and BA was reached, ignoring chain ends, the average number of NIPAM repeat units between chemically bonded cross-links, $N_{c}$ can be approximated by

$$
N_{C}=\frac{1}{2 M_{\text {NPAM }}\left(C_{B A} / M_{B A}\right)}
$$

where $M_{\text {NIPAM }}$ is the molecular weight of NIPAM repeat unit, $C_{B A}$ is the concentration of the cross-linker in the unit of wt\% of NIPAM and $M_{B A}$ is the molecular weight of the cross-linker. At $25^{\circ} \mathrm{C}$ and for $\mathrm{M}-1, \mathrm{M}-2$ and $\mathrm{M}-3$ microgels with BA addition of 2, 6 and $10 w \%$, the number of NIPAM repeat units between cross-links was 34 for $\mathrm{M}-1,11.4$ for $\mathrm{M}-2$ and 6.8 for $\mathrm{M}-3$.

Wu et al's work on the kinetics of PNIPAM microgel formation [33] indicated that the crosslinker, $N, N^{\prime}$-methylenebisacrylamide, was consumed slightly faster than $N$-isopropylacrylamide, as shown in Figure 1 in Reference 33. Therefore, it is proposed that M-4 prepared through batch polymerization had 
non-uniform structure and long dangling PNIPAM chains. Meanwhile, M-1, M-2 and M-3 synthesized through semi-batch polymerization were expected to have relatively more uniform structures and shorter dangling chains. $\mathbf{M}-1, \mathbf{M}-2$ and $\mathbf{M}-$ 3 were different from each other in crosslinking degrees. Although BA added amount and therefore the averaged $N_{c}$ was the same for both $M-3$ and M-4, BA was incorporated into the M-3 microgel networks more uniformly due to the continuous supply of BA in a semi-batch polymerization. Because of faster consumption of BA [33], the actual $N_{c}$ in the cores of all the microgels was speculated to be smaller than its calculated values. It was also found that, at 70 ${ }^{\circ} \mathrm{C}$ and within $20 \mathrm{~min}$ of the initiation of polymerization, the monomer conversion of both BA and NIPAM was nearly complete and the diameter of the microgels as a function of time leveled off [33]. Therefore, within $150 \mathrm{~min}$ of polymerization, both NIPAM and BA were completely consumed and incorporated into microgel networks. The amount of BA as weight percentage of NIPAM in a microgel was the same as it was in the feed (Table 5. 3).

In summary, M-4 had least uniform in structure with total crosslinker amount of 10 wt\% of NIPAM. M-1, M-2 and M-3 had relatively more uniform structure with total crosslinker amount of 2,6 and $10 w t \%$ of NIPAM in each kind of microgels. Dangling chains on M-4 spheres were speculated to be the longest among the four kinds of microgels. 
Define the swelling ratio of the microgels as $\phi_{\text {melling }}=\frac{R_{h, 25 C}}{R_{h, 40 C}}$, the values of the swelling ratio is calculated and listed in Table 5. 3. M-1 had the greatest swelling with the least amount of crosslinker. $M-2$ and $M-3$ had the same $\phi_{\text {swelling, }}$ suggesting these two kinds of microgels had similar swelling behavior.

McPhee et al [4] found that the volume phase transition of BA crosslinked microgels occurred between 30 and $34^{\circ} \mathrm{C}$ when BA composition varied from 0 to $15 w \%$ of NIPAM. Therefore, it is concluded that at $25^{\circ} \mathrm{C}$, all the four kinds of microgels existed in swollen non-phase separated state and at $40^{\circ} \mathrm{C}$, they all shrunk and phase-separated. The dynamic surface tension measurements at these two temperatures studied the behaviors before and after the volume phase transition. Table 5. 4 shows $\gamma_{m}$ was neither sensitive to crosslinker concentration nor to temperature, while $t^{*}$ was greatly influenced by chemical crosslinking and by polymer chain aggregation due to volume phase transition.

The ESEM micrograph in Figure 5. 2 is the first published example of an order array of colloidal particles at the air/water interface which lower surface tension. There are many published examples of monolayers of particulate materials, however, they do not lower surface tension. Both crosslinking and deswelling affect the mobility of polymer chains. There are two limiting cases of adsorption at the air/water interface. One is the adsorption of surface active linear PNIPAM. Linear PNIPAM chains are the most mobile. When become adsorbed 
at the air/water interface, linear PNIPAM are able to unwrapped and lower the surface tension of water down to a meso-equilibrium value of about $43 \mathrm{~mJ} / \mathrm{m}^{2}$ [50]. The other case is the adsorption of rigid solid spherical particles. Those rigid solid spheres normally do not lower the surface tension of water and corresponds to an air/water interface covered with a monolayer of spheres. Adsorption of crosslinked PNIPAM microgels has similar effect to the adsorption of linear PNIPAM in lowering the surface tension of water, while their arrangement at the air/water interface resembles the adsorption of rigid spheres. Figure 5. 3, Figure 5. 4 and Figure 5. 5 show the time scales and amplitudes of the surface tension lowering processes of the microgels. The ESEM picture displayed in Figure 5.2 clearly demonstrates the orderly manner of the spherical microgels (M-3) actually adsorbed at the air/water interface.

Assuming the microgels adsorbed at the air/water interface are rigid spheres, a Langmuir kinetics model $[51,70]$ is developed to help to explain the influence of crosslinker concentration on the microgel surface tension lowering ability. Assuming that there is no desorption:

$$
\frac{d \theta}{d t}=k_{1}\left(n_{0}-\theta\right)(1-\theta) \quad \text { Equation } 5.8
$$

where, $k_{l}$ is a constant in the unit of $\mathrm{s}^{-1}, t$ is the time in $\mathrm{s}, \theta$ is the coverage of the interface and is defined as 


$$
\theta=\frac{\Gamma}{\Gamma_{\max }}
$$

Equation 5.9

$\Gamma$ is the surface concentration of the microgels at the air/water interface in $\mathrm{mg} / \mathrm{m}^{2}$, $\Gamma_{\max }$ is the maximum capacity of the surface to adsorb polymer in $\mathrm{mg} / \mathrm{m}^{2}$. The mass of a microgel can be calculated using experimental data at $40^{\circ} \mathrm{C}$ :

$$
m_{g e l}=\frac{4}{3} \pi\left(R_{n, \infty 0 c}\right)^{3} C_{p}
$$

$R_{h, 40 C}$ is found in Table 5. 3. $C_{p}$ is the polymer concentration in a microgel. Dong and Hoffman reported water content in crosslinked PNIPAM macrogel as function of temperature [38]. The water content at $40^{\circ} \mathrm{C}$ read from Figure 4 in Reference 38 is 30 wt\%. Assume all the BA crosslinked PNIPAM gels have the same water content after phase separation, ignoring the crosslinker influence, then all the gels have the same polymer concentration within them at $40^{\circ} \mathrm{C} . C_{P}$ is 0.7 $\mathrm{g} / \mathrm{cm}^{3}$, converted from the water content value at $40^{\circ} \mathrm{C}$. Assuming that rigid spheres have cubic packing at the interface, then

$$
\Gamma_{\max }=\frac{\frac{4}{3} \pi\left(R_{A, 40 C}\right)^{3} C_{p}}{4 R_{n}^{2}}=\frac{\pi\left(R_{h, 40 C}\right)^{3} C_{P}}{3 R_{h}{ }^{2}}
$$

where $R_{k}$ is the hydrodynamic radius at $25^{\circ} \mathrm{C}$ in $\mathrm{cm}$ (refer to Table 5. 3), if modeling is done at $25^{\circ} \mathrm{C} . n_{o}$ in Equation 5.8 is defined as the ratio of the initial 
number of polymer molecules to the maximum number of polymer molecules that can adsorb onto the available surface, which is equivalent to

$$
n_{0}=\frac{C_{0} V}{\Gamma_{\max } A}=\frac{C_{0} \varepsilon}{\Gamma_{\max }}
$$

where $C_{0}$ is the initial microgel mass concentration in a suspension in $\mathrm{mg} / \mathrm{cm}^{3}, V$ is the volume of the pendent drop in $\mathrm{cm}^{3}, A$ is the drop surface area in $\mathrm{cm}^{2}$ and $\varepsilon$ is the drop V/A ratio in $\mathrm{cm}$, which is a known experimental parameter. $n_{0}$ for the four 1 wt\% microgel suspensions are calculated to be between 178 and 322 (See Appendix 5.3). Since the maximum value of $\theta$ is one, therefore $\left(n_{0}-\theta\right)$ in Equation 5.8 is approximately equal to $n_{0}$ for $1 w t \%$ concentration level, which indicates depletion in the bulk phase is insignificant, then Equation 5.8 is reduced to

$$
\frac{d \theta}{d t}=k_{1} n_{0}(1-\theta)
$$

The boundary condition for Equation 5.12 is $\theta=0$ when $t=0$. Solving Equation 5.12,

$$
1-\theta=\exp \left(-k_{1} n_{0} t\right)
$$

Equation 5.13

Assuming surface tension is a simple function of coverage:

$$
\gamma=\gamma_{m}+\left(\gamma_{0}-\gamma_{m}\right)(1-\theta)
$$


where, $\gamma$ is the dynamic surface tension, $\gamma_{m}$ is the meso-equilibrium surface tension and $\gamma_{0}$ is the surface tension of pure water.

Finally, substitute Equation 5.10, Equation 5.11 and Equation 5.13 into Equation 5.14, we have

$$
\gamma=\gamma_{m}+\left(\gamma_{0}-\gamma_{m}\right) \exp \left(-\frac{3 k_{1} C_{0} \varepsilon R_{h}^{2}}{\pi\left(R_{h, \Delta 0 C}\right)^{3} C_{p}} t\right)
$$

Figure 5. 4 shows the experimental dynamic surface tensions of 1 wt\% microgel suspensions at $25^{\circ} \mathrm{C}$ and the corresponding prediction of Equation 5.15 with $k_{1}$ chosen to be $0.000163 \mathrm{~s}^{-1}$, so the predictions to the dynamic surface tension of M-2 are approximately correct. $\gamma_{m}$ and $C_{0}$ taken from Table 5. 4, $R_{h}$ and $R_{h, 40 C}$ taken from Table 5. 3. The $t^{*}$ values for the four model curves shown in Figure 5. 4 are calculated and listed under $t_{M}$ in Table 5. 4 with "M" denoting the "Model". This one parameter Langmuir model gives the surface tension lowering ability order as $M-1>M-2>M-4>M-3$, judging from how long it takes for the dynamic surface tension to approach its meso-equilibrium value. This predicted order agrees with the surface tension lowering order concluded from the experimental results. Both the predicted and experimental orders of surface tension lowering are consistent with the order of $R_{h}$ values, $M-1<M-2<M-4<M-3$. The fact that the smaller the hydrodynamic radius of a microgel the faster the surface tension lowering process indicates that diffusion of the microgels to the 
air/water interface influence the surface tension process. While the prediction of the surface tension lowering order is correct, the model was not successful at predicting the time scale for the dynamic surface tension change. Using only one adjustable parameter $\left(k_{1}\right)$, the model can only fit well to the experimental curve of M-2. The failure of the model may be because of over-simplification of the actual situation by assuming rigid microgel spheres and ignoring the contribution of dangling PNIPAM chains in lowering surface tension. All the polymer chains in a water-swollen microgel are very mobile. However, chains restricted by crosslinks can not as easily spread out to occupy bare air/water interface as linear homopolymer PNIPAM chains can. In such a way, crosslinking strongly influenced the mobility of the polymer chains in the microgels. The microgels are not rigid, although crosslinking affects the polymer chain mobility. And the dangling chains, which are more mobile than the chains between crosslinks, can spread out at the air/water interface and contribute to the surface tension lowering.

The fundamental source of the surface-active properties of the NIPAM polymers is the $N$-isopropyl (IP) groups on NIPAM moieties. It is speculated that the surface concentration of IP groups at the air/water interface dictates the surface tension change in the adsorption of linear PNIPAM homopolymer, poly( $N$-isopropylacrylamide-co-acrylamide) copolymers and crosslinked PNIPAM microgels. IP group content in an NIPAM monomer molecule is 38.08 wt\%. To see the IP concentration at the air/water interface, the empirical 
relationship between surface tension and surface concentration of adsorbed linear PNIPAM is used. Using Kawaguchi et al's experimental data [71], this empirical relationship between surface tension and surface concentration was developed in the previous work (see Appendix 2.5) as

$$
\gamma_{2 S C}\left(\Gamma_{\text {NPAM }}\right)=42.19+\frac{29.95}{1+2.10 \times 10^{-6} e^{21.7829 \Gamma_{\text {NPRN }}}}
$$

Equation 5.16

Rearranging Equation 5.16 and converting $\Gamma_{\text {NIPAM }}$ into $\Gamma_{I P}$, the equivalent surface concentration of IP groups, gives

$$
\Gamma_{I P}(\gamma)=0.2285+0.0175 \operatorname{Ln} \frac{72.14-\gamma}{\gamma-42.19}
$$

Equation 5.17

Equation 5.17 shows the surface concentration of IP groups as a function of surface tension at $25^{\circ} \mathrm{C}$. Using the meso-equilibrium surface tension data in the previous work (Table 2, 50), the maximum meso-equilibrium surface concentration of IP groups for linear PNIPAM adsorbed at the air/water interface is calculated to be $0.31 \mathrm{mg} / \mathrm{m}^{2}$ at $25^{\circ} \mathrm{C}$. Using $\gamma_{m}$ listed in Table 5.4 and Equation 5.17, the meso-equilibrium equivalent surface concentration $\left(\Gamma_{1 P, m}\right)$ of IP groups for the four microgel samples at $25^{\circ} \mathrm{C}$ are calculated (see Appendix 5.4) and shown in Table 5. 4. $\Gamma_{I P, m}$ is $0.27 \mathrm{mg} / \mathrm{m}^{2}$ for both $1 \mathrm{wt} \% \mathrm{M}-2$ and 1 wt\% M-3 and $0.28 \mathrm{mg} / \mathrm{m}^{2}$ for both $0.05 \mathrm{wt} \% \mathrm{M}-1$ and $1 \mathrm{wt} \% \mathrm{M}-4$. It is interesting that $\Gamma_{I P, m}$ for $1 \mathrm{wt} \% \mathrm{M}-1$ has the same value of $0.31 \mathrm{mg} / \mathrm{m}^{2}$ as the maximum meso- 
equilibrium surface concentration of L-6B. From those calculation results, it is clear that the meso-equilibrium surface tensions of the microgel suspensions were not very sensitive to the crosslinking degrees although the kinetics is, and similar amount of IP groups were presented at the air/water interface for either linear PNIPAM polymer or microgels.

The surface-active role of IP groups similar to lowering surface tension of water has been seen in other cases of polymer adsorbed at the air/water or hydrophobic surface/water interface. Asada et al's work on the surface-active properties of poly( $N$-alkylsubstituted acrylamide)s and their adsorption onto polystyrene latex particles in water indicated that the adsorption of those polymers at the air/water interface and PS particle/water interface was mainly governed by the overall hydrophobicity and the hydrophobicity of their alkyl groups of $\mathbf{N}$-alkylacrylamide units. Our previous work on copolymers of NIPAM and acrylamide also found that an aqueous solution of poly $(N$ isopropylacrylamide-co-acrylamide) with lower NIPAM fraction, i.e. aqueous copolymers of lower $\boldsymbol{N}$-isopropyl group content, had higher surface tension as a result of diluting $N$-isopropyl group's surface concentration at the air/water interface when the copolymer was adsorbed [53].

Figure 5. 7 presents a speculated picture showing how NIPAM polymers lower the surface tension of water. Linear homopolymers and crosslinked microgels should first diffuse to the air/water interface, then polymer chains more 
or less unwrap and spread out to cover the air/water interface, with hydrophobic $N$-isopropyl groups preferably protruding into the air phase, and lower the surface tension of water. It is proposed that less crosslinked NIPAM polymers occupy the air water interface more efficiently because of higher polymer chain mobility. For example, among all the hydrated or dehydrated NIPAM polymers, PNIPAM coils before phase separation has the smallest hydrodynamic radius in the order of $10^{-2} \mathrm{~nm}[69]$ and the greatest polymer chain mobilities. As a result, aqueous linear PNIPAM homopolymers before the phase separation has the fastest surface tension lowering (see Figure 5. 4, Figure 5. 5, and Table 2.2 for example).

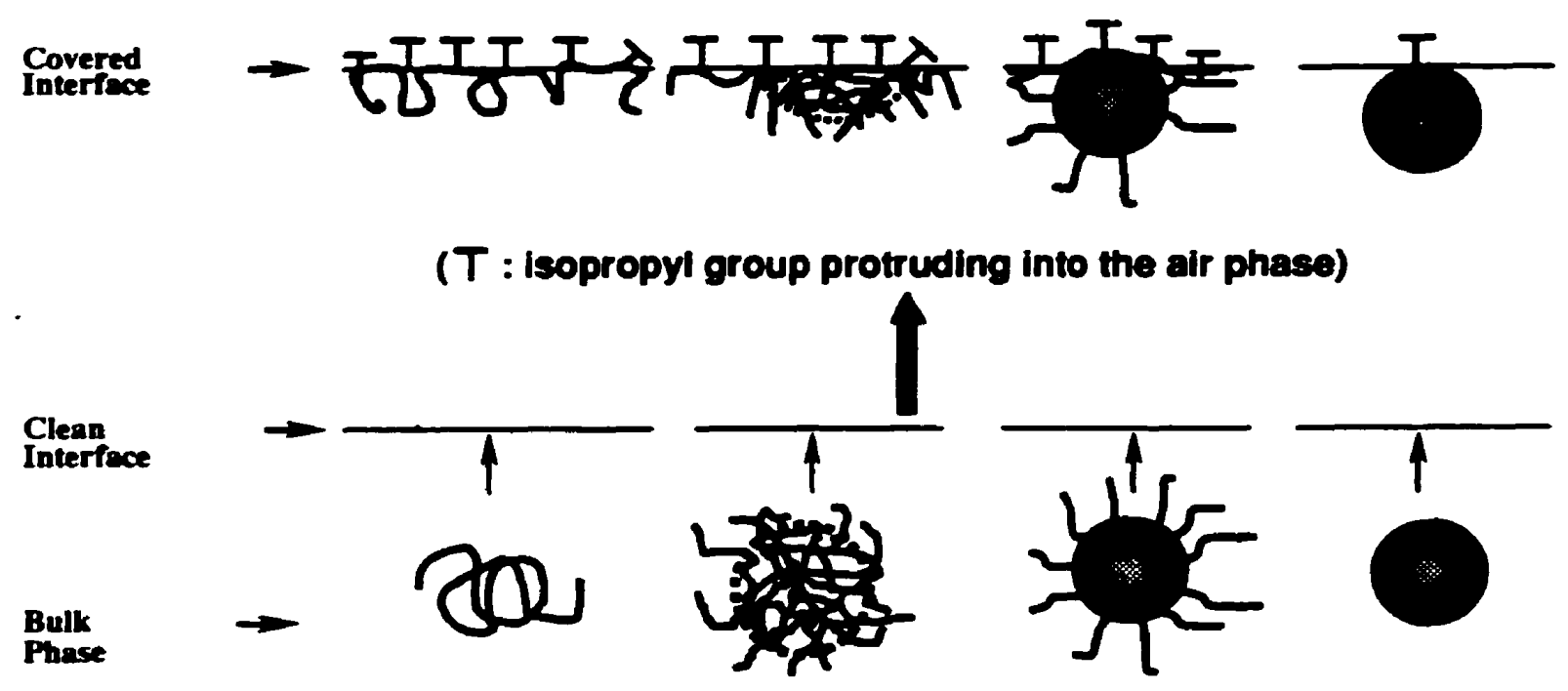

Figure 5. 7 A proposed picture showing how NIPAM polymers lower the surface tension of water before the phase separation for linear polymers or volume phase transition of the microgels. 
Phase-separated homopolymer particles and dehydrated microgels display similar surface tension lowering behaviors to the scenario shown in Figure 5. 7. The difference of the homopolymer case is that phase-separated PNIPAM diffuse to the air/water interface as colloidally stable particles other than individual random coil, with slower diffusion to the air/water interface and reduced polymer chain mobility. Whereas, dehydrated microgels have smaller hydrodynamic radii than swollen microgels (Table 5. 3) and their diffusion to the air/water interface from the bulk phase is faster than the swollen ones. However, the faster diffusion is traded off with the severely reduced mobility of polymer chains between crosslinks by both chemical crosslinking and dehydration. As a result, the surface tension lowering processes of dehydrated microgels are slower than waterswollen ones as manifested by the $t^{*}$ values (Figure 5. 6 and Table 5. 4). After arrival at the air/water interface, dehydrated homopolymers chains released from the particles, unwrap, spread to occupy the interface and lower the surface tension of water with the hydrophobic $N$-isopropyl groups. For dehydrated microgels arriving the interface, some dangling polymer chains or chains between crosslinks more or less rearrange or partially unwrap, to cover the interface with the $N$ isopropyl groups and lower the surface tension of water. 


\subsection{Conclusions}

1. PNIPAM microgels adsorb at the air/water interface and lower the surface tension of water.

2. The rate of surface tension lowering dramatically decreases with increasing crosslinker concentration in microgels. In the extreme, linear polymer was much faster than crosslinked microgels in lowering the surface tension of water. The aggregation of polymer chains caused by deswelling through volume phase transition also slowed down the surface tension lowering process

3. The meso-equilibrium surface tension values are not very sensitive to either temperature or crosslinking.

4. Microgels prepared by batch polymerization gave faster surface tension lowering than those prepared by semi-batch. 


\subsection{Acknowledgements}

The authors acknowledge Professor Neumann and Dr. Yu Hing Daniel Kwok at University of Toronto, for allowing us to use their surface tension apparatus. The authors also thank the Canadian Natural Science and Engineering Research Council for funding. 


\subsection{References}

1 Pelton, R.H. and Chibante, P., Colloids and Surfaces, 20, 247 (1986).

2 Schild, H. G., Prog. Polym. Sci., 17, 163 (1992).

3 Osada, Y. and Ross-Murphy, S. B., Scientific American, May, 82 (1993).

4 McPhee, W.; Tam, K. C. and Pelton, R. H., J. Colloid Interface Sci., 156, 24 (1993).

5 Bae,Y. H.; Okano, T. and Kim, S. W., J. of Polym. Sci.: Part B: Polym. Phys., 28, 923 (1990).

6 Tanaka, T. and Fillmore, D. J., J. Chem. Phys., 70, 1214 (1979).

7 Tanaka, T.; Sato, E.; Hirokawa, Y.; Hirotsu, S. and Peetermans, J., Phys. Rev. Lett., 55, 2455 (1985).

8 Matsuo, E. S.; Tanaka, T., J. Chem. Phys., 89, 1695 (1988).

9 Tam, K.C.; Ragaram, S. and Pelton, R.H., Langmuir, 10, 418 (1994).

10 Pelton, R. H.; Pelton, H. M.; Morphesis, A. and Rowell, R. L., Langmuir, 5, 816 (1989).

11 Mears, S. J.; Deng, Y.; Cosgrove, T. and Pelton, R., Langmuir, 13, 1901 (1997).

12 Wang, G.; Pelton, R. and Zhang, Ju, accepted by Colloids and Surfaces.

13 Sakai ,M.; Satoh, N.; Tsujii, K.; Zhang, Y. and Tanaka, T., Langmuir, 11 , 2493 (1995).

14 Kokufuta, E.; Suzuki, H. and Sakamoto, D., Langmuir, 13, 2627 (1997).

15 Shirahama, K.; Sato, S.; Niino, M. and Takisawa, N., Colloids and Surfaces A: Physicochemical and Engineering Aspects, 112, 233 (1996).

16 Murray, M.; Rana, F.; Haq, I.; Cook, J.; Chowdhry, B. Z. and Snowden, M. J., J. Chem. Soc., Chem. Commun., 1803 (1994). 
17 Tanaka, T., Phys. Rev. Lett., 40, 820, (1978).

18 Tanaka, T., Phys. Rev. Lett., 45, 1636 (1980).

19 Katayama, S.; Hirokawa, Y.; Tanaka, T., Macromolecules, 17, 2641 (1984).

20 Otake, K.; Inomata, H.; Konno, M. and Saito, S., Macromolecules, 23, 283 (1990).

21 Yoshida, R.; Uchida, K.; Kaneko, Y.; Sakai, K.; Kikuchi, A.; Sakurai, Y. and Okano, T., Nature, 374, 240 (1995).

22 Saunders, B. R.; and Vincent, B., J. Chem. Soc. Faraday Trans, 92, 3385 (1996).

23 Lele, A. K.; Hirve, M. M.; Badiger, M. V. and Mashelkar, R. A., Macromolecules, 30, 157 (1997).

24 Shibayama, M.; Zutani, S. M. and Nomura, S., Macromolecules, 29, 2019 (1996).

25 Pankasem, S.; Thomas, J. K.; Snowden, M. J. and Vincent, B., Langmuir, 10 , 3023 (1994).

26 Snowden, M. J.; Chowdhry, B. Z; Vincent, B.; Morris, G. E., J. Chem. Soc. Faraday Trans., 92, 5013 (1996).

27 Kawasaki, H.; Sasaki, S.;and Maeda, H., J. Phy. Chem. B, 101, 4184 (1997).

28 Kawasaki, H.; Sasaki, S. and Maeda, H., Maromolecules, 30, 1847 (1997).

29 Yoshida, R.; Sakai, K.; Okano, T.; Sakurai, Y.; Bae, Y. H. and Kim, S. W., J. Biomater. Sci. Polymer Edn., 3, 155, (1991).

30 Yoshida, R.; Sakai, K.; Okano, T. and Sakurai, Y., J. Biomater. Sci. Polymer Edn., 3, 243, (1992).

31 Yoshida, R.; Sakai, K.; Okano, T. and Sakurai, Y., J. Biomater. Sci. Polymer Edn., 6, 585 (1994).

32 Kaneko, Y.; Sakai, K.; Kikuchi, A.; Yoshida, R.; Sakurai, Y. and Okano, T., Macromolecules, 28, 7717 (1995). 
33 Wu, X.; Pelton, R. H.; Hamielec, A. E.; Woods, D. R. and McPhee, W., Colloid Poly. Sci., 272, 467 (1994).

34 Murray, M.; Charlesworth, D.; Swires, L.; Riby, P.; Cook, J.; Chowdhry, B. Z. and Snowden, M. J., J. Chem. Soc. Faraday Trans., 90, 1999 (1994).

35 Wang, C. H. and Cao, W. X., Polymer International, 41, 449 (1996).

36 Hoffman, A. S.; Afrassiabi, A. and Dong, L. C., J. Controlled Release, 4, 213 (1986).

37 Dong, L. C. and Hoffman, A. S., J. Controlled Release, 4, 223 (1986).

38 Dong, L. C. and Hoffman, A. S., J. Controlled Release, 13, 21 (1990).

39 Wu, X. S.; Hoffman, A. S. and Yager, P., J. Polym. Sci.: Part A: Polym. Chem., 30, 2121 (1992).

40 Park T. G. and Hoffman, A. S., J. Applied Polym. Sci., 46, 659 (1992).

41 Dong, L. C. and Hoffman, A. S., J. Controlled Release, 15, 141 (1991).

42 Okuyama, Y.; Yoshida, R.; Sakai, K; Okano, T. and Sakurai, Y., J. Biomater. Sci. Polymer Edn., 4, 545 (1993).

43 Okano, T.; Bae, Y. H.; Jacobs, H. and Kim, S. W., J. Controlled Release, 11, 255 (1990).

44 Takahashi, F.; Sakai, Y.; Mizutani, Y., J. Fermentation and Bioengineering, 83, 152 (1997).

45 Wang, K. L.; Burban, J. H.; Cussler, E. L.; in Responsive Gels: Volume Transitions II (Ed. K. Dusek), Berlin: Springer, P.68,1993.

46 Kanazawa, H.; Yamamoto, K.; Kashiwase, Y.; Matsushima, Y.; Takai, N.; Kikuchi, A.; Sakurai, Y. and Okano, T., J. Pharmaceutical and Biomedical Analysis, 15, 1545 (1997).

47 Kanazawa, H.; Kashiwase, Y.; Yamamoto, K.; Matsushima, Y.; Kikuchi, A.; Sakurai, Y. and Okano, T., Analytical Chemistry, 69, 823 (1997). 
48 Weissman, J. M.; Sunkara, H. B.; Tse, A. S. and Asher, S. A., Science, 274, 959 (1996).

49 Zhang, Ju and Pelton, R., Langmuir, 12, 2611 (1996).

50 Zhang, Ju and Pelton, R., "The dynamic behavior of poly $(N$ isopropylacrylamide) at the Air/Water Interface", accepted by Colloids and Surfaces.

51 Zhang, Ju, and Pelton, R, "The application of polymer adsorption models to dynamic surface tension", submitted to Lngmuir.

52 Chan, K.; Pelton, R. and Zhang, Ju, "On the Formation of Colloidally Dispersed Phase-separated Poly( $N$-isopropylacrylamide)", submitted to Langmuir.

53 Zhang, Ju and Pelton, R., "The Surface Tension of Aqueous Poly $(N$ isopropylacrylamide-co-acrylamide)", accepted by J. Polym. Sci. Part A: Polymer Chemistry.

54 Jasper, J. J., J. Physical and Chemical Reference Data, 1, 948 (1972).

55 a) Brookhaven Variable Angle QELS Manual Appendix: Software Options, Pages A-1 to A-11; b). Provencher, S. W., Makromol. Chem. 180, 201 (1979); c) Brown, J. C.; Pusey, P. N. and Dietz, R., J. Chem. Phys., 62, 1136 (1975).

56 Burchard, W., Adv. Polym. Sci. 48, 1, (1983).

57 Hiemenz, P. C., Principles of Colloid and Surface Chemistry, Marcel Dekker, Inc., New York and Basel, 1997.

58 Kratochvil, P., Classical Light Scattering from Polymer Solutions, Elsevier, Amsterdam, 1987.

59 Seebergh, J. E.; Berg, J. C., Colloids and Surfaces A: Physichemical and Engineering Aspects, 100, 139 (1995).

60 Rotenberg, Y.; Boruvka, L.; Neumann, A. W., J. Colloid Interface Sci., 93, 169 (1983).

61 Kwok, Daniel Yu Hing, Masters Thesis, University of Toronto, 1994. 
62 Hua, X. Y. and Rosen, M. J., J. Colloid Interface Sci., 124, 652 (1988).

63 Hua, X. Y. and Rosen,M. J., J. Colloid Interface Sci., 141, 180 (1991).

64 Schmidt, M.; Nerger, D. and Burchard, W., Polymer, 20, 582 (1979).

65 Kunz, D.; Thum, A. and Burchard, W., Colloid Polym. Sci. 261, 635 (1983).

66 Kunz, D. and Burchard, W., Colloid Polym. Sci., 264, 498 (1986).

67 Nieuwenhuis, E. A.; Pathmamanoharan, C. and Vrij, A., J. Colloid Interface Sci., 81196 (1981).

68 Wolfe, M. S. and Scopazz, C., J. Colloid Interface Sci., 133, 1265 (1989).

69 Wu, C. and Zhou, S., Macromolecules, 28, 8381 (1995).

70 van de Ven, T.G.M., Advances Colloid Interface Sci., 48, 121 (1994).

71 Kawaguchi, M.; Saito, W. and Kato, T., Macromolecules, 27, 5882 (1994). 


\section{Appendix 5.1 Extrapolations of Eff. Diam. $\left(D_{E}\right)$ to Zero Angle and Zero Concentration at $25^{\circ} \mathrm{C}$}

The objective of this spreadsheet is to extrapolate the intensity-

weighted mean hydrodynamic equivalent spherical diameters $\left(D_{E}\right)$ of $M-1$

microgel, which were measured as functions of incident angle $(\theta)$ and

concentration (C) through dynamic light scattering (DLS) measurements, to the angle of zero degree and the concentration of zero $\mathrm{ppm}$.

The method of cumulants analysis results was used and the Eff. Diam. $\left(D_{E}\right)$ was recorded as the hydrodynamic equivalent spherical diameter [1]

1. Fix the angle and extrapolate $D_{E}$ to zero concentration:

55 deg.

C (ppm)

$50 \quad 100$

150

200

$D_{E}(\mathbf{n m})$

$\begin{array}{lll}757.4667 & 756.7667\end{array}$

705.425

709.75

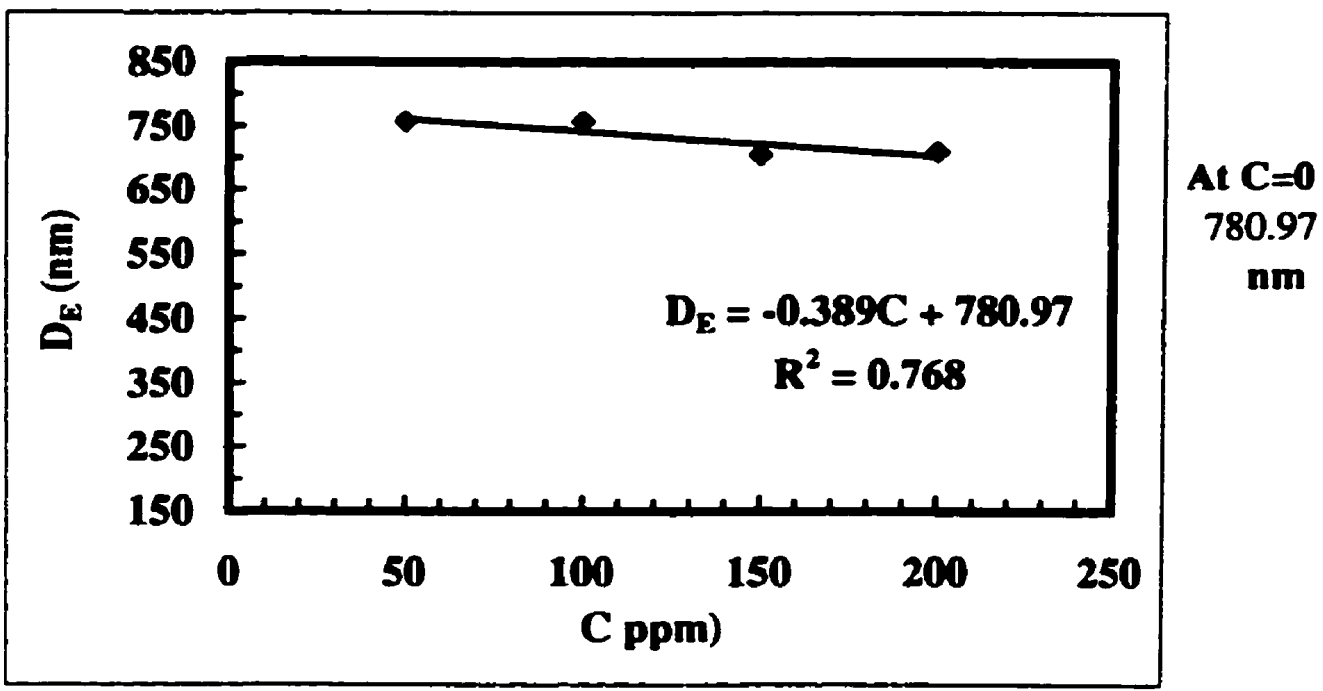


2. Extrapolate $D_{E}$ at $C=0$ ppm to angle of zero degree:

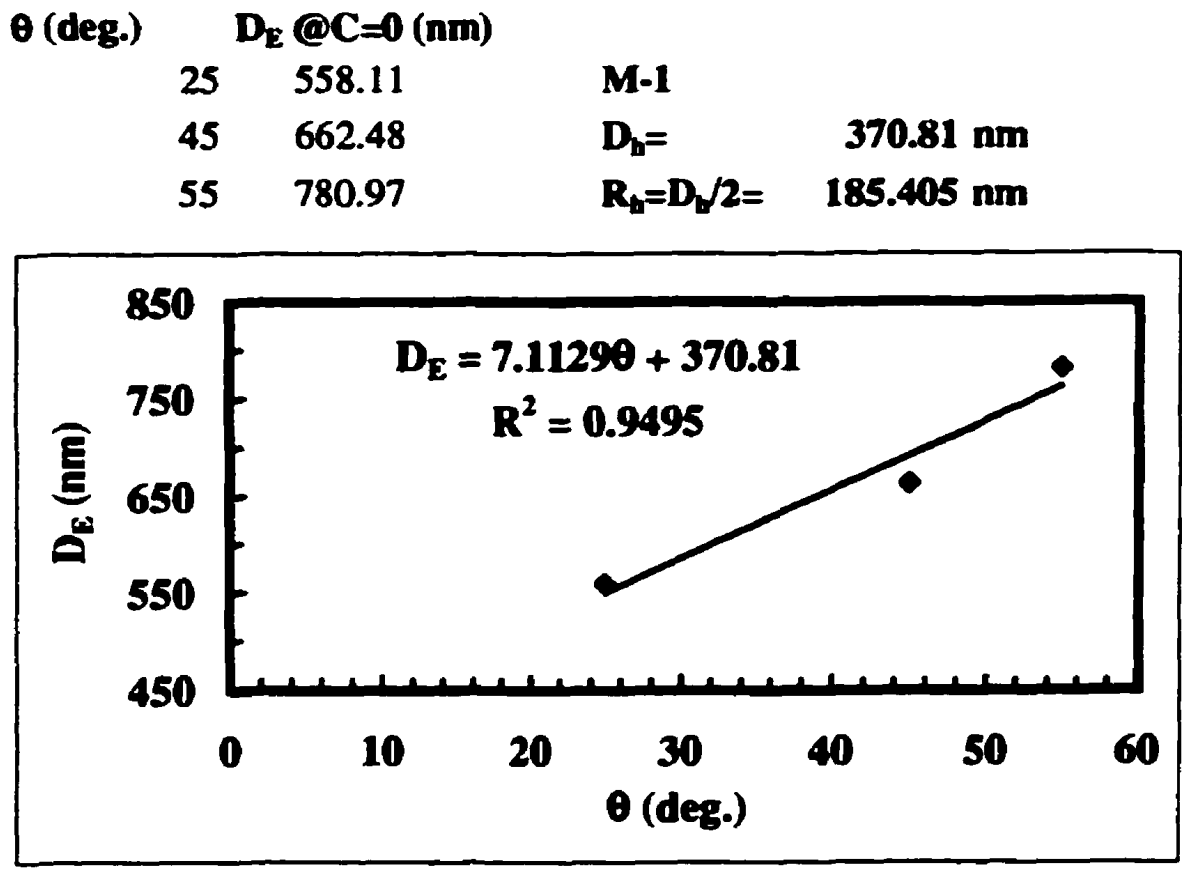


The objective of this spreadsheet is to extrapolate the intensity-weighted mean hydrodynamic equivalent spherical diameters $\left(D_{E}\right)$ of M-2 microgel, which were measured as functions of incident angle $(\theta)$ and concnetration (C) through dynamic light scattering (DLS) measurements, to the angle of zero degree and the concentration of zero ppm.

The method of cumulants analysis results was used and the Eff. Diam.(DE) was recorded as the hydrodynamic equivalent spherical diameter [1].

1. Fix the angle and extrapolate $D_{E}$ to zero concentration:

An example of the extrapolation at $25^{\circ} \mathrm{C}$.

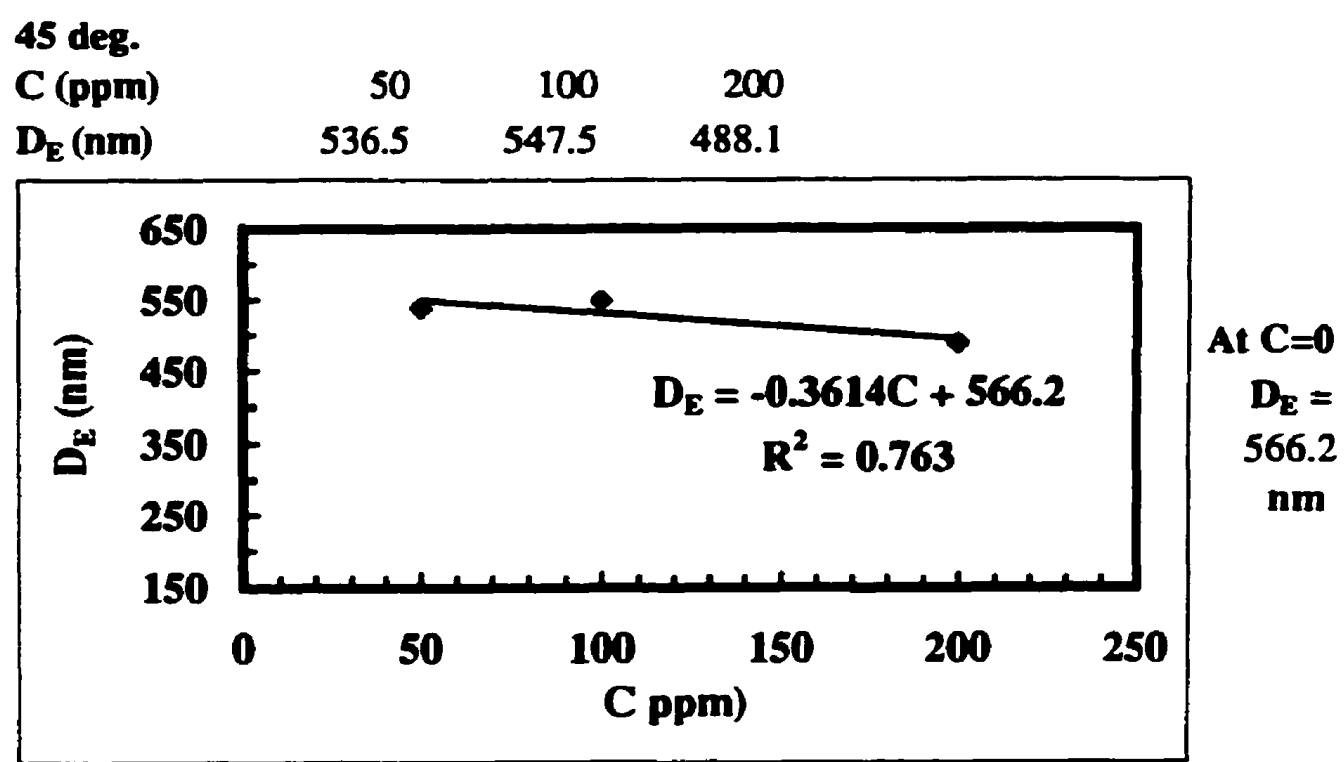

Diameter at 90 degree was determined by measurement at an very dilute solution $(50 \sim 100 \mathrm{kcnt} / \mathrm{s})$ :

$q=90$ deg.

$\mathrm{D}_{\mathrm{E}}(\mathbf{c m})$

593.2

591.6

596.6

594.3

592.7

$593.7 \quad 598.6$

average $\quad 594.3857 \mathrm{~nm}$

STDEV $\quad 2.42035 \mathrm{~nm}$ 
2. Extrapolate $D_{E}$ at $C=0 \mathrm{ppm}$ to angle of zero degree:

$\theta$ (deg.) $D_{E} @ C=0(\mathrm{~nm})$

$\begin{array}{rrll}35 & 560 & M-2 & \\ 45 & 566 & D_{b}= & 538.28 \mathrm{~mm} \\ 55 & 573.57 & R_{b}=D_{\downarrow} / 2= & 269.14 \mathrm{~nm} \\ 90 & 594.4 & & \end{array}$

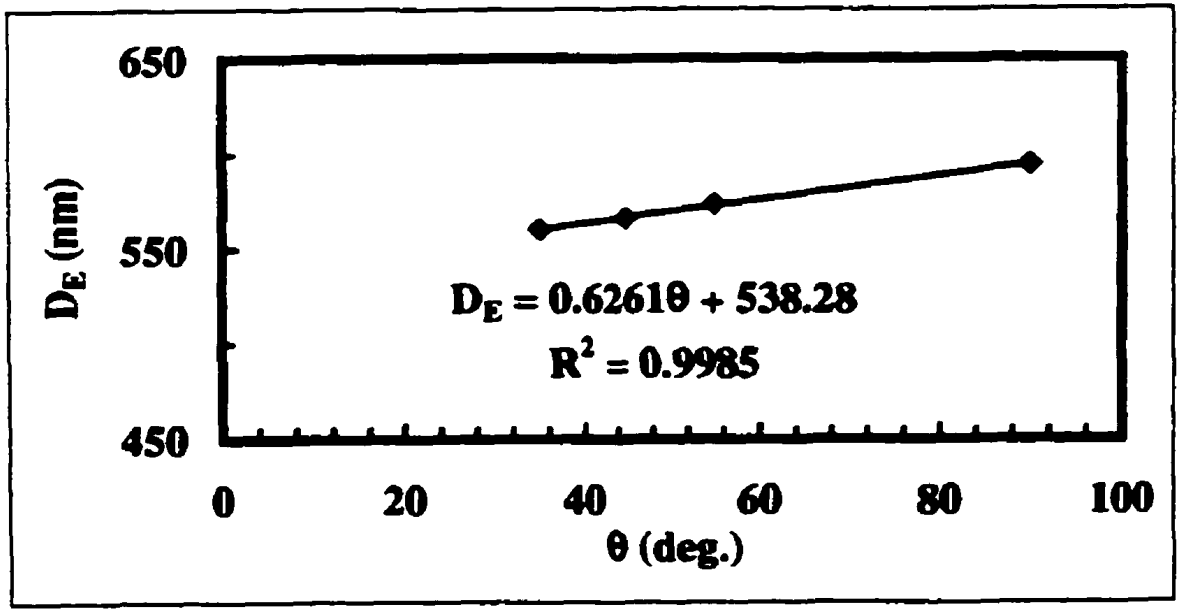


The objective of this spreadsheet is to extrapolate the intensity-

weighted mean hydrodynamic equivalent spherical diameters $\left(D_{\mathcal{E}}\right)$ of $M-3$

microgel, which were measured as functions of incident angle $(\theta)$ and concnetration (C) through dynamic light scattering (DLS) measurements, to the angle of zero degree and the concentration of zero ppm.

The method of cumulants analysis results was used and the Eff. Diam.( $\left(D_{E}\right)$ was recorded as the hydrodynamic equivalent spherical diameter [1]

1. Fix the angle and extrapolate $D_{E}$ to zero concentration:

An example of the extrapolation at $25^{\circ} \mathrm{C}$.

35 deg.

C (ppm)

$4.04 \quad 7.86$

20

30

$\begin{array}{lllll}D_{E}(n m) & 647.2333 & 659.1667 & 643 & 604.6667\end{array}$

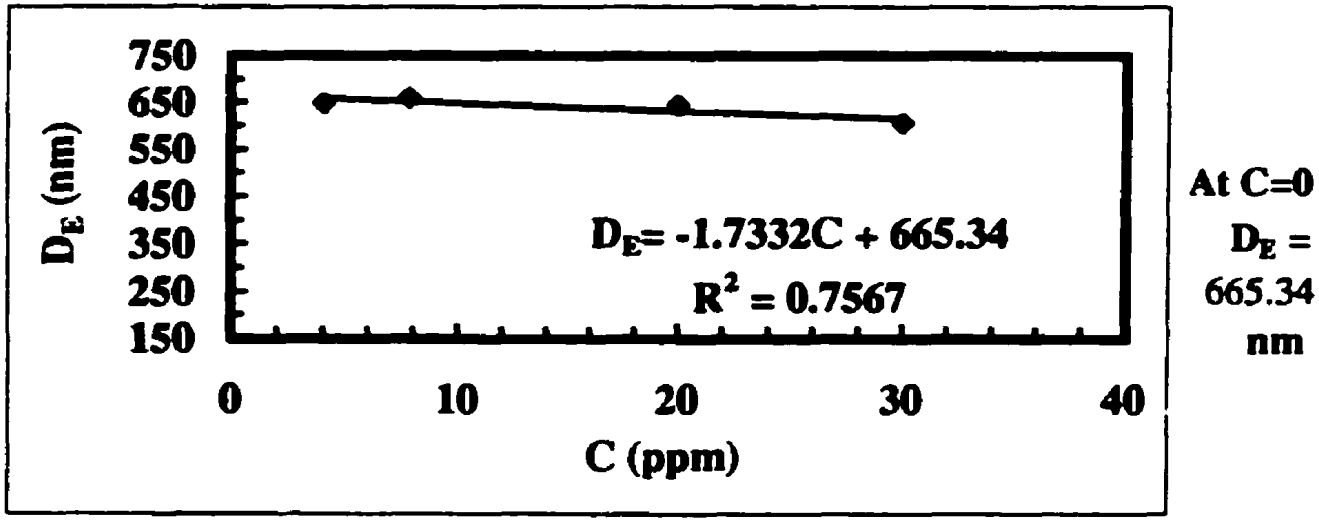

2. Extrapolate $D_{E}$ at $C=0 \mathrm{ppm}$ to angle of zero degree:

$q$ (deg.) $\quad D_{E} @ C=0(n m)$

$\begin{array}{rrlr}25 & 661.7 & D_{b}= & 636.51 \mathrm{~nm} \\ 35 & 665.34 & R_{\mathrm{b}}=D_{\mathrm{w}} / 2= & 318.255 \mathrm{~nm} \\ 45 & 680.34 & & \end{array}$

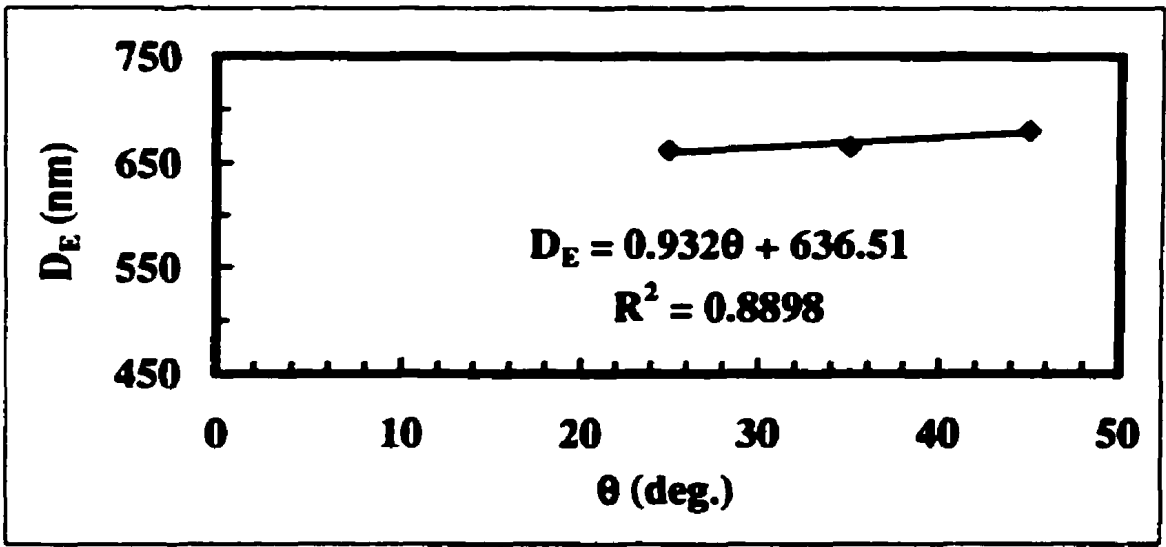


The objective of this spreadsheet is to extrapolate the intensity-weighted mean hydrodynamic equivalent spherical diameters $\left(D_{E}\right)$ of $M-4$ microgel, which were measured as functions of incident angle $(\theta)$ and concnetration (C) through dynamic light scattering (DLS) measurements, to the angle of zero degree and the concentration of zero $\mathrm{ppm}$.

The method of cumulants analysis results was used and the Eff. Diam.( $\left.\mathrm{D}_{\mathrm{E}}\right)$ was recorded as the hydrodynamic equivalent spherical diameter [1]

1. Fix the angle and extrapolate $D_{E}$ to zero concentration:

25 deg.

C (ppm)

5

10

15

$\begin{array}{llll}D_{E}(\mathrm{~nm}) & 477.48 & 305.55 & 213.2\end{array}$

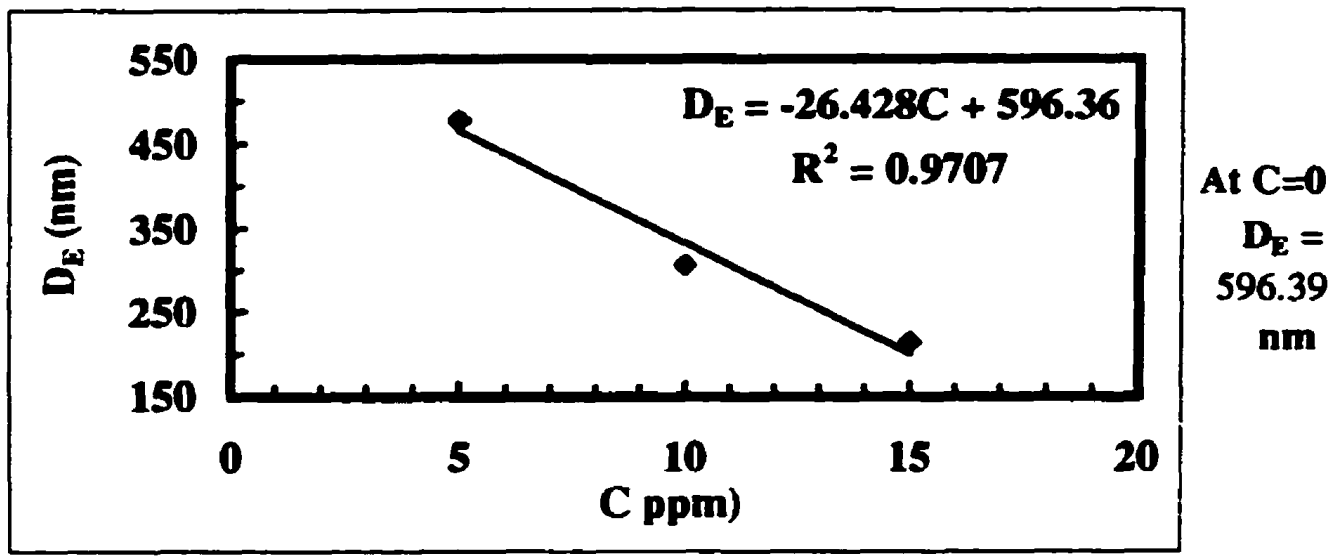

2. Extrapolate $D_{E}$ at $C=0 \mathrm{ppm}$ to angle of zero degree:

$\theta$ (deg.) $\quad D_{E} @ C=0(\mathrm{~nm})$

$\begin{array}{rrlr}25 & 596.3 & D_{b}= & 594.75 \mathrm{~nm} \\ 45 & 602.64 & R_{b}=D_{k} / 2= & 297.375 \mathrm{~nm} \\ 55 & 598.375 & & \end{array}$

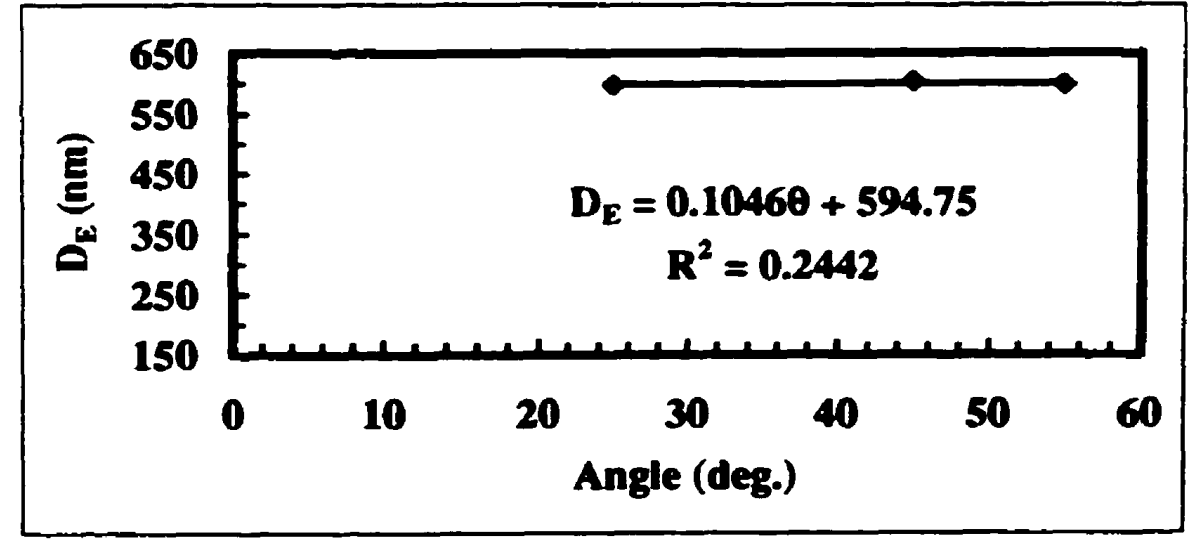




\section{Reference:}

1. a) Brookhaven Variable Angle QELS Manual Appendix: Software Options, Pages A-1 to A-1 1; b). Provencher, S. W., Makromol. Chem ., 180, 201 (1979); c) Brown. J. C.; Pusey, P. N. and Dietz, R., J. Chem. Phys., 62, 1136 (1975). 


\section{Appendix 5.2 The Dissymmetry Method $-\mathbf{R}_{\mathrm{g}}$}

The objective of this document is to find $R g$ through processing the intensity data obtained in static light scattering (SLS) experiment, using the dissymmetry method [1].

1. The Dark Counts (DK) at different angles ( $\theta$ )

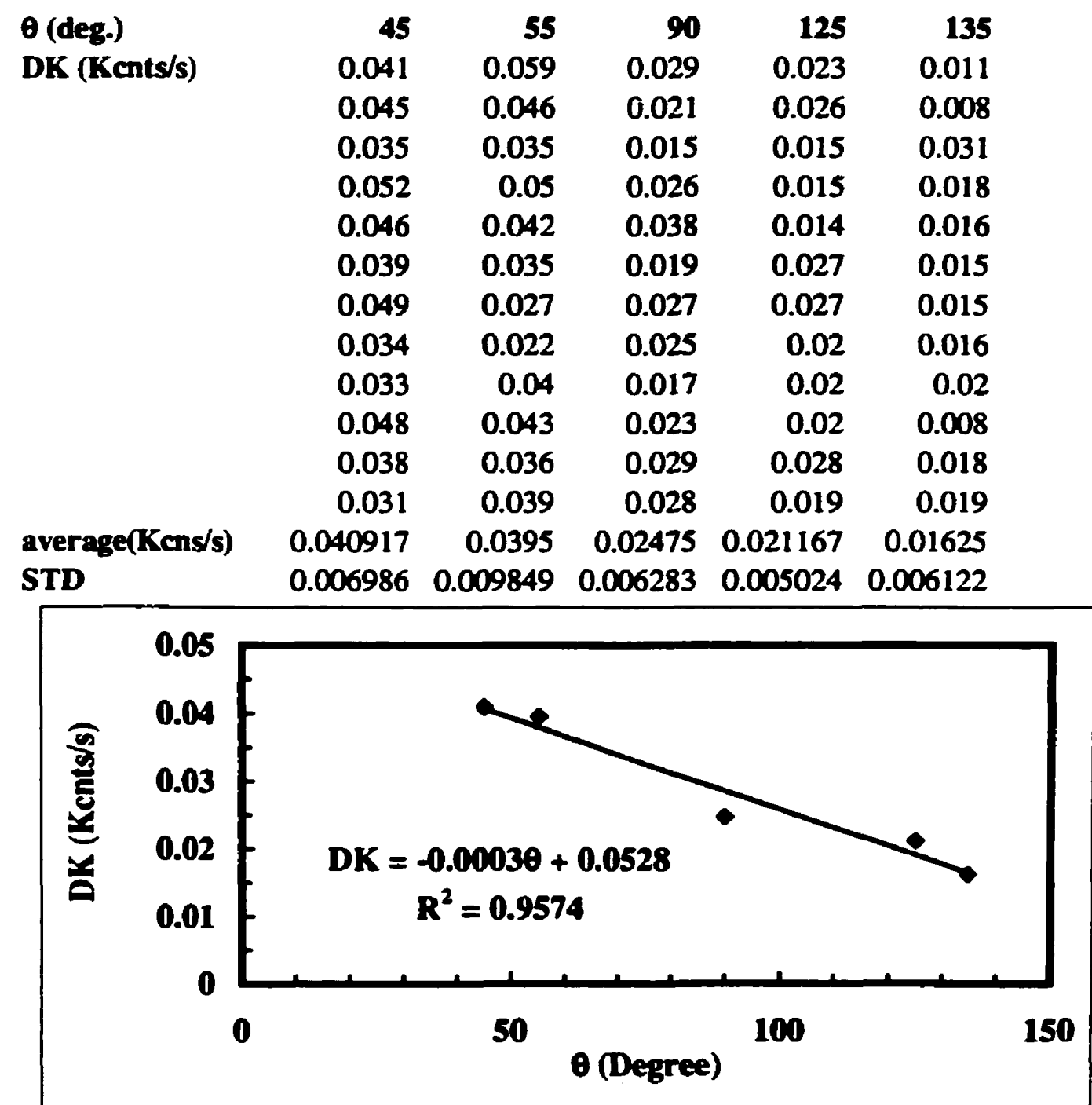


2. The Intensity of Water (IW) at difierent angles

\begin{tabular}{lrrrrr}
\hline (deg.) & 45 & 55 & 90 & 125 & 135 \\
IW (Kents/s) & 1.285 & 1.641 & 1.181 & 1.342 & 1.511 \\
& 1.32 & 1.581 & 1.115 & 1.397 & 1.482 \\
& 1.243 & 1.546 & 1.108 & 1.265 & 1.617 \\
& 1.235 & 1.493 & 1.124 & 1.313 & 1.482 \\
& 1.294 & 1.455 & 1.253 & 1.291 & 1.448 \\
& 1.309 & 1.473 & 1.081 & 1.281 & 1.493 \\
& 1.279 & 1.552 & 1.036 & 1.331 & 1.486 \\
& 1.22 & 1.492 & 1.106 & 1.319 & 1.546 \\
& 1.301 & 1.478 & 1.121 & 1.313 & 1.471 \\
& 1.288 & 1.512 & 1.11 & 1.372 & 1.503 \\
& & 1.525 & 1.14 & 1.308 & 1.592 \\
& & 1.587 & 1.169 & 1.302 & 1.525 \\
average(Kcns/s) & 1.2774 & 1.527917 & 1.128667 & 1.3195 & 1.513 \\
STD & 0.03349 & 0.055267 & 0.054246 & 0.037159 & 0.04994
\end{tabular}

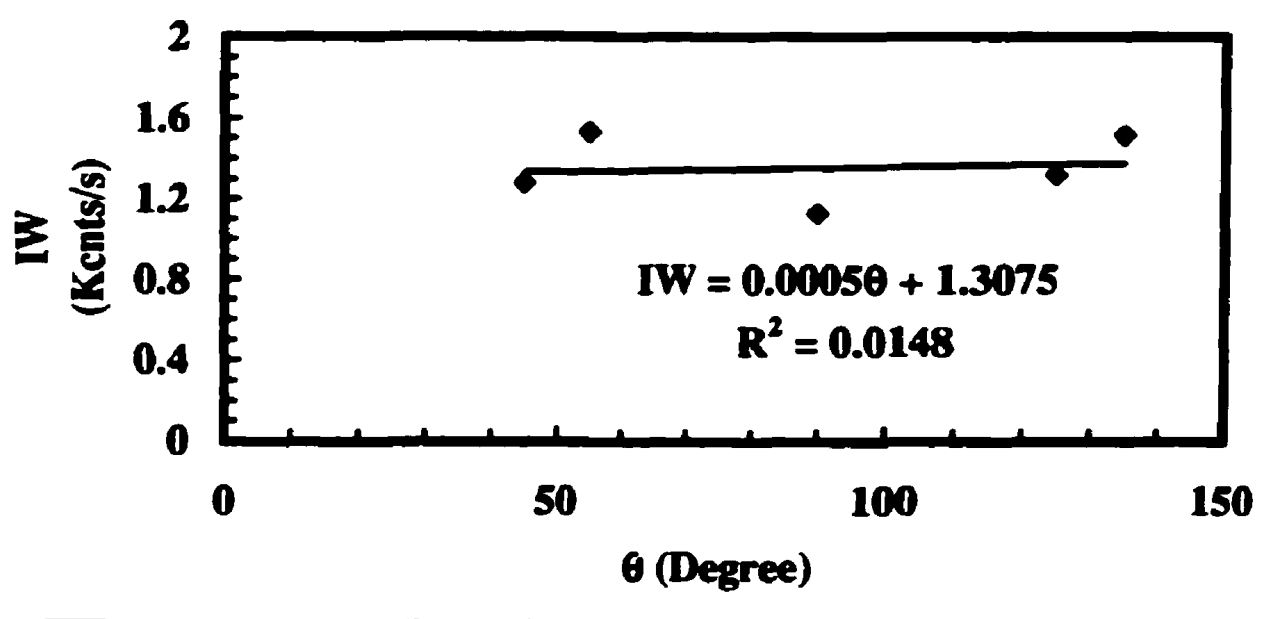


3. The Dissymmetric Ratio (Z) and Rg of M-1

$$
Z=\frac{I_{\theta}}{I_{(120-\theta)}}
$$

Refractive Index of solvent water at $25^{\circ} \mathrm{C}$

$$
\mathrm{n}=\quad \mathbf{1 . 3 3 6}
$$

\begin{tabular}{|c|c|c|c|}
\hline \multirow{11}{*}{$\begin{array}{l}\theta \text { (deg.) } \\
I_{0}(\mathrm{Kcnts} / \mathrm{s})\end{array}$} & 45 & 90 & 135 \\
\hline & 178.28 & 11.979 & 34.215 \\
\hline & 212.246 & 11.694 & 34.48 \\
\hline & 189.506 & 12.322 & 33.61 \\
\hline & 169.079 & 12.718 & 33.05 \\
\hline & 187.439 & 12.523 & 32.85 \\
\hline & 172.629 & 11.912 & 35.09 \\
\hline & 193.696 & 12.284 & 34.5 \\
\hline & 182.446 & 12.132 & 34.008 \\
\hline & 185.187 & 11.842 & 34.79 \\
\hline & 190.585 & 12.729 & 33.27 \\
\hline Io,average(Kens/s) & 186.1093 & 12.2135 & 34.0373 \\
\hline STD & 12.11675 & 0.364115 & 0.812659 \\
\hline DK & 0.040917 & 0.02475 & 0.01625 \\
\hline IW & 1.2774 & 1.128667 & 1.513 \\
\hline$I_{0}-$-DK-IW & 184.791 & 11.06008 & 32.50805 \\
\hline $\mathbf{I} \boldsymbol{d}_{(\mathbf{1}}$ & 5684468 & 1 & 0.175918 \\
\hline C (ppm) & $z$ & & \\
\hline & 5.684468 & & \\
\hline & 7.683446 & & \\
\hline & 8.833511 & & \\
\hline & 9.668546 & & \\
\hline & 10.48554 & & \\
\hline
\end{tabular}

An example of the calculation of $\mathbf{I}_{(160)} \mathbf{I}_{(50} \mathrm{ppm}$

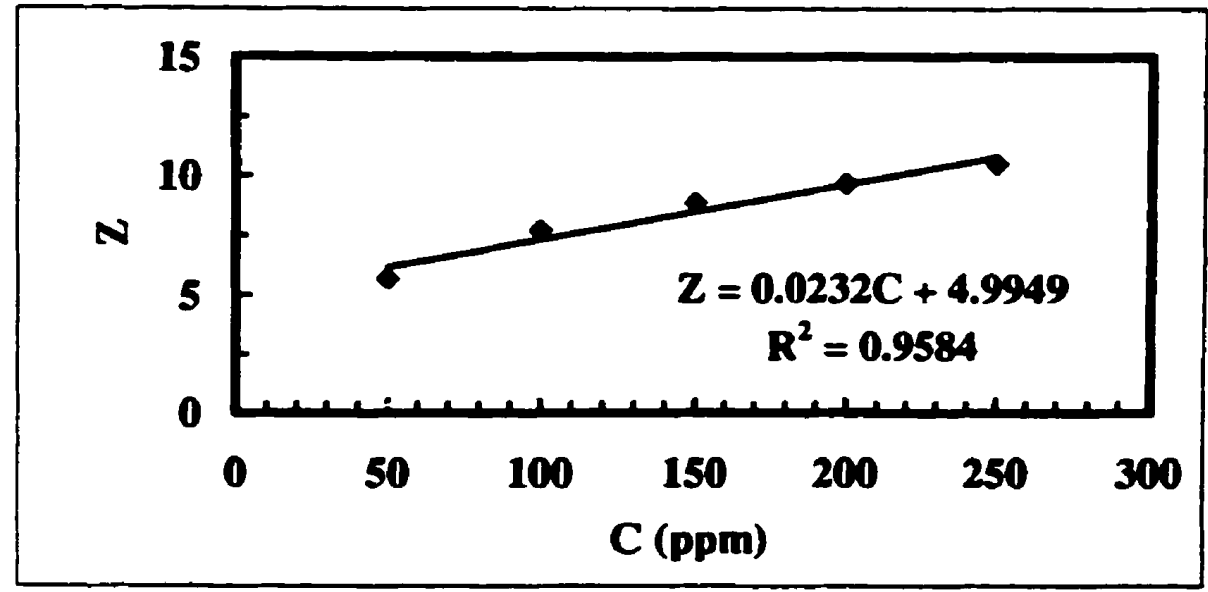


$\frac{I_{\theta}}{I_{(180-\theta)}}=\frac{P(\theta)}{P(180-\theta)}$

$P(\theta)=\left[\left(\frac{3}{x^{3}}\right)(\sin x-x \cos x)\right]^{2}$

$\theta$ (deg.)

$x=2 \pi\left(\frac{D n}{\lambda_{0}}\right) \sin \left(\frac{\theta}{2}\right)$

$\lambda_{0}=514 \mathrm{~nm} \quad \mathrm{n}=1.336$

135

$$
\begin{aligned}
& \mathbf{L}_{\mathbf{4}} / \mathbf{I}_{135} \\
& \mathbf{Z}=
\end{aligned}
$$

$x_{45}$

$x_{135}$

$P(45)=$

$\mathbf{P}(135)=$

Z-P(45)/P(135)=

4.9949
-1.43708
-2.94693
0.653097
0.130753
0.00

$\mathrm{D}=$$$
R^{2}=\frac{3}{5}\left(\frac{D}{2}\right)^{2}
$$

$180.7141 \mathrm{~nm}$

$\mathbf{R g}=$

$69.99027 \mathrm{~nm}$

To find D: use Solver to find a D which satisfies Z-P(45)/P(135)= 0 . 
4. The Dissymmetric Ratio (Z) and Rg of M-2

$$
Z=\frac{I_{\theta}}{I_{(120-\theta)}}
$$

Refractive Index of solvent water at $25^{\circ} \mathrm{C}$

$$
\mathrm{n}=\quad \mathbf{1 . 3 3 6}
$$

An example of the calculation of $\mathbf{W} \mathbf{I}_{(120,0)}: 50 \mathrm{ppm}$

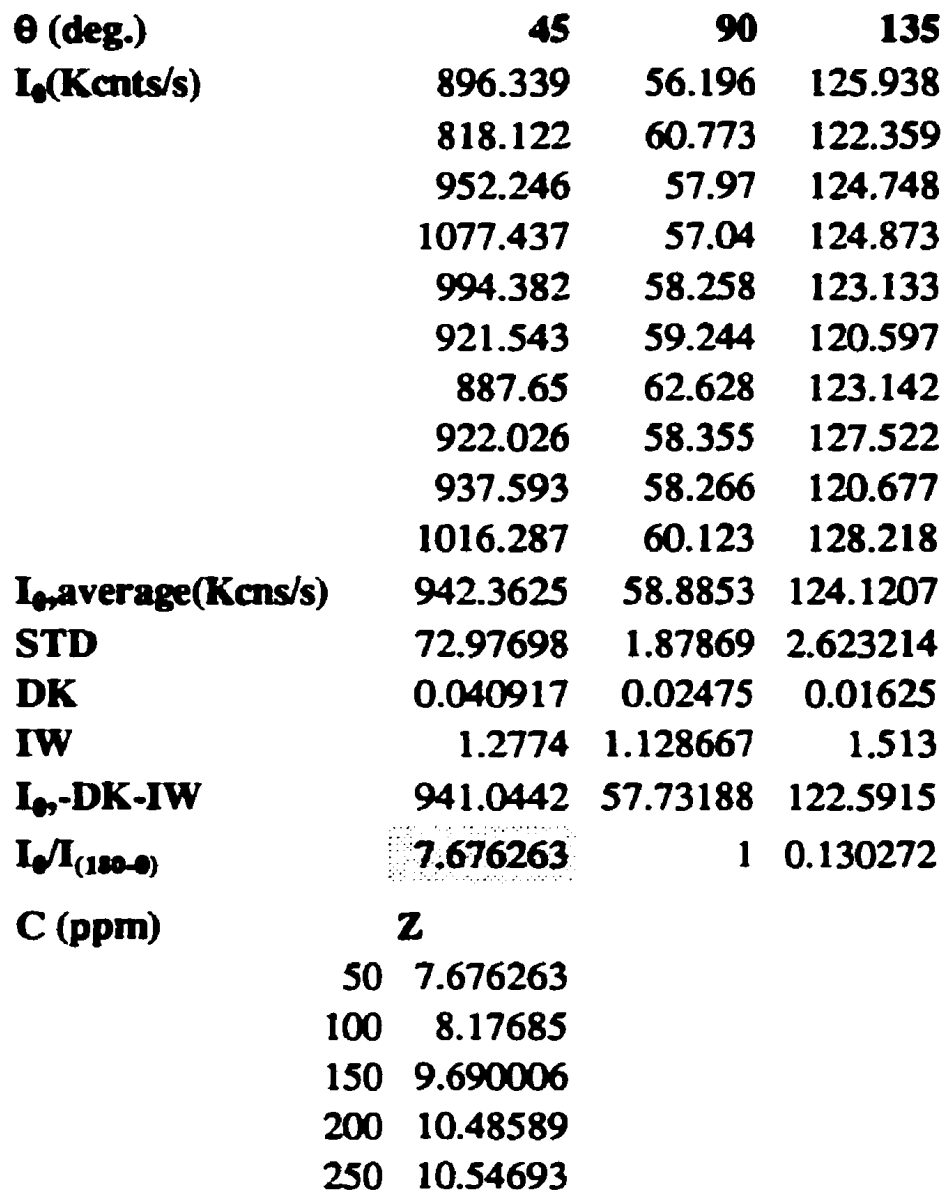

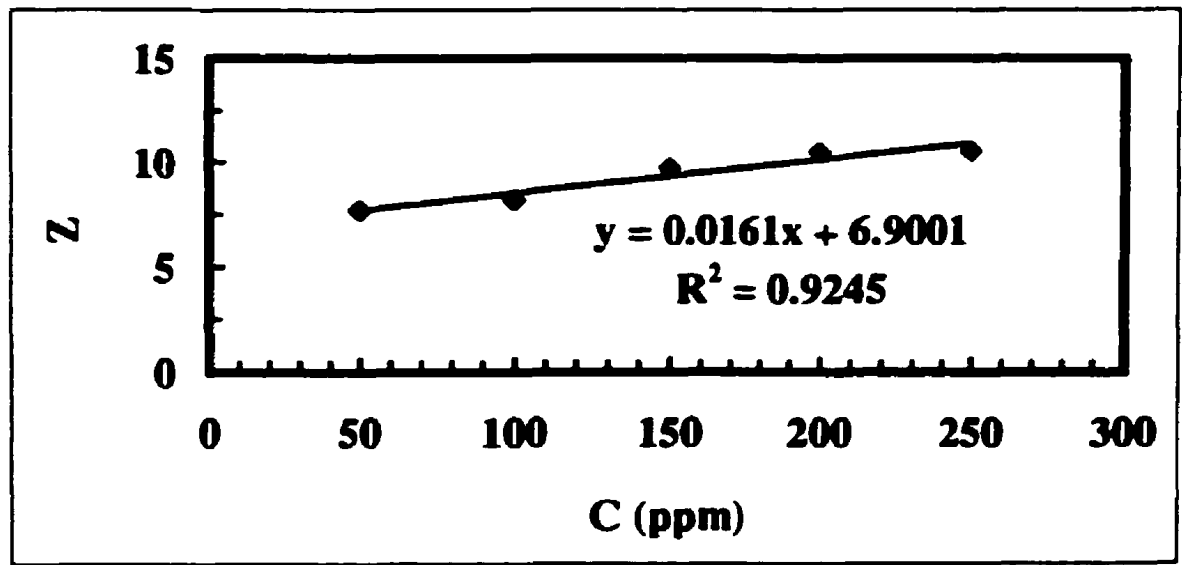




$$
\frac{I_{\theta}}{I_{(180-\theta)}}=\frac{P(\theta)}{P(180-\theta)}
$$$$
x=2 \pi\left(\frac{D n}{\lambda_{0}}\right) \sin \left(\frac{\theta}{2}\right)
$$

$L_{45} I_{135}$

$z=$

$x_{45}$

$x_{135}$

$P(45)=$

$P(135)=$

Z-P(45)/P(135)=

$$
P(\theta)=\left[\left(\frac{3}{x^{3}}\right)(\sin x-x \cos x)\right]^{2}
$$$$
\lambda_{0}=514 \mathrm{~nm} \quad \mathrm{n}=1.336
$$

$\theta$ (deg.)

45

135
$\mathbf{D}=$

$$
R_{8}{ }^{2}=\frac{3}{5}\left(\frac{D}{2}\right)^{2}
$$

$194.0354 \mathrm{~nm}$

$-1.54301$

$-3.16416$

0.610411

0.088464

$\mathrm{Rg}=\quad 75.14959 \mathrm{~nm}$

To find D: use Solver to find a D which satisfies Z-P(45)/P(135)=0. 
5. The Dissymmetric Ratio (Z) and Rg of M-3

$$
Z=\frac{I_{\theta}}{I_{(120-0)}}
$$

Refractive Index of solvent water at $25^{\circ} \mathrm{C}$

$$
\mathrm{n}=
$$

\begin{tabular}{|c|c|c|c|}
\hline \multirow{13}{*}{$\begin{array}{l}\theta \text { (deg.) } \\
\text { Io(Kents/s) }\end{array}$} & 45 & 90 & 135 \\
\hline & 207.218 & 19.586 & 19.962 \\
\hline & 210.376 & 15.212 & 19.711 \\
\hline & 260.386 & 15.308 & 20.44 \\
\hline & 224.714 & 16.51 & 24.45 \\
\hline & 256.24 & 15.319 & 20.881 \\
\hline & 246.918 & 16.031 & 20.165 \\
\hline & 230.704 & 16.937 & 19.534 \\
\hline & 274.247 & 14.472 & 22.272 \\
\hline & 270.86 & 14.564 & 20.853 \\
\hline & 249.722 & 15.084 & 20.186 \\
\hline & 315.17 & 14.859 & 19.653 \\
\hline & 252.291 & 14.835 & 19.984 \\
\hline Io,average $(\mathrm{Kcns} / \mathrm{s})$ & 249.9038 & 15.72642 & 20.67425 \\
\hline STD & 29.9147 & 1.432063 & 1.40425 \\
\hline DK & 0.040917 & 0.02475 & 0.01625 \\
\hline IW & 1.2774 & 1.128667 & 1.513 \\
\hline $\mathbf{I}_{0},-\mathbf{D K}-\mathbf{I W}$ & 248.5855 & 14.573 & 19.145 \\
\hline $\mathbf{I}_{\mathbf{d}} \mathbf{I}_{(180-0)}$ & 1298436 & 1 & 0.077016 \\
\hline$C(p p m)$ & $\mathbf{z}$ & & \\
\hline 4.04 & 12.98436 & & \\
\hline 7.86 & 13.72625 & & \\
\hline 10 & 13.8662 & & \\
\hline 30 & 14.71812 & & \\
\hline
\end{tabular}

An example of the calculation of $\mathrm{I}_{\mathbf{1}} \mathrm{I}_{(1000)}: 4.04 \mathrm{ppm}$

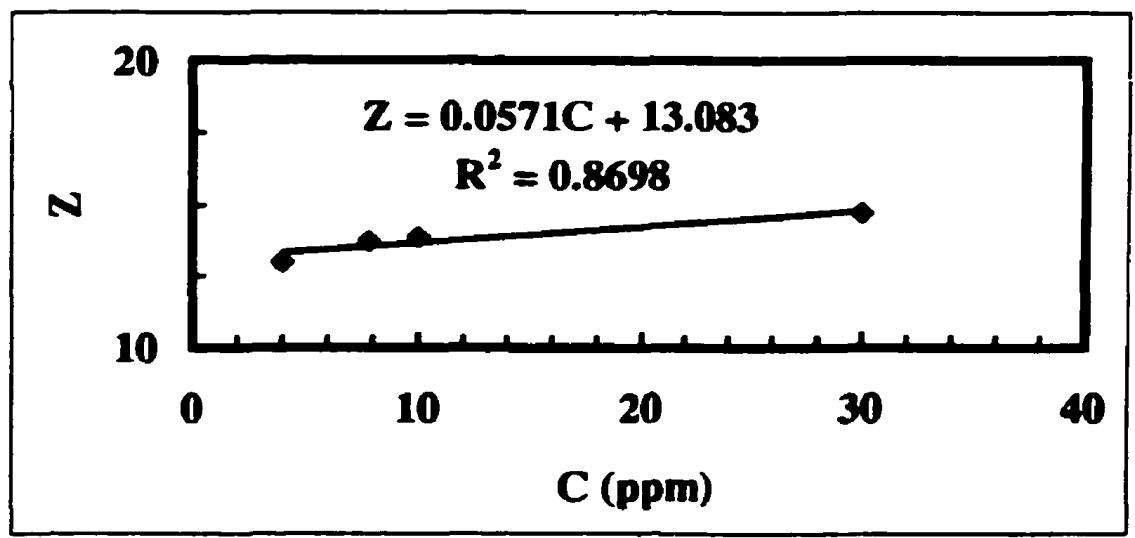




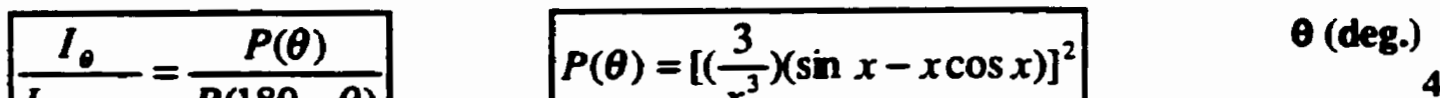

$$
\begin{aligned}
& \left.\frac{I_{(180-\theta)}}{I_{(180-\theta)}}=\frac{P(\theta)}{P\left(P(\theta)=\left[\left(\frac{3}{x^{3}}\right)(\sin x-x \cos x)\right]\right.}\right] \quad 45 \\
& x=2 \pi\left(\frac{D n}{\lambda_{0}}\right) \sin \left(\frac{\theta}{2}\right) \quad n=1.336
\end{aligned}
$$

$$
\begin{aligned}
& I_{45} / I_{135} \\
& Z=
\end{aligned}
$$

$\times 45$

$x_{135}$

$P(45)=$

$\mathbf{P}(\mathbf{1 3 5})=$

Z-P(45)/P(135)=

$\begin{array}{ll}13.083 & \mathrm{D}= \\ -1.70878 & \\ -3.5041 & R_{z}{ }^{2}=\frac{3}{5}\left(\frac{D}{2}\right)^{2} \\ 0.542968 & \mathrm{Rg}= \\ 0.041502 & 83.22325 \mathrm{~nm}\end{array}$

To find D: use Solver to find a $D$ which satisfies Z-P(45)/P(135)= 0 . 
6. The Dissymmetric Ratio (Z) and $\operatorname{Rg}_{\mathrm{g}}$ of $\mathrm{M}-4$

$$
Z=\frac{I_{\theta}}{I_{(180-\theta)}}
$$

Refractive Index of solvent water at $25^{\circ} \mathrm{C}$

$$
\mathbf{n}=
$$

1.336

An example of the calculation of $I_{\boldsymbol{\alpha}} \boldsymbol{I}_{(100,0)}: 5 \mathrm{ppm}$

$\theta$ (deg.)

$I_{0}(K e n t s / s)$
45

867.468

898.02

954.107

995.012

912.504

913.477

1025.332

907.042

844.763

907.87

Io,average(Kcns/s)

STD

DK

IW

$I_{0},-D K \cdot I W$

$\mathbf{I} / \mathbf{I}_{(\mathbf{1 0 0})}$

C (ppm)

922.5595

54.91056

0.040917

1.2774

921.2412

19.40999

Z

90

135

$48.539 \quad 50.248$

$45.686 \quad 50.469$

45.589

49.041

45.212

47.012

46.051

49.265

48.181

47.937

44.988

49.832

50.131

48.333

45.937

49.484

47.768

48.181

46.9432

48.8452

1.833539

1.198721

0.02475

0.01625

1.128667

1.513

45.78978

l 0.051361
$\begin{array}{ll}5 & 19.46999\end{array}$
$10 \quad 18.74655$
$15 \quad 19.85712$
$\begin{array}{ll}20 & 20.63242\end{array}$

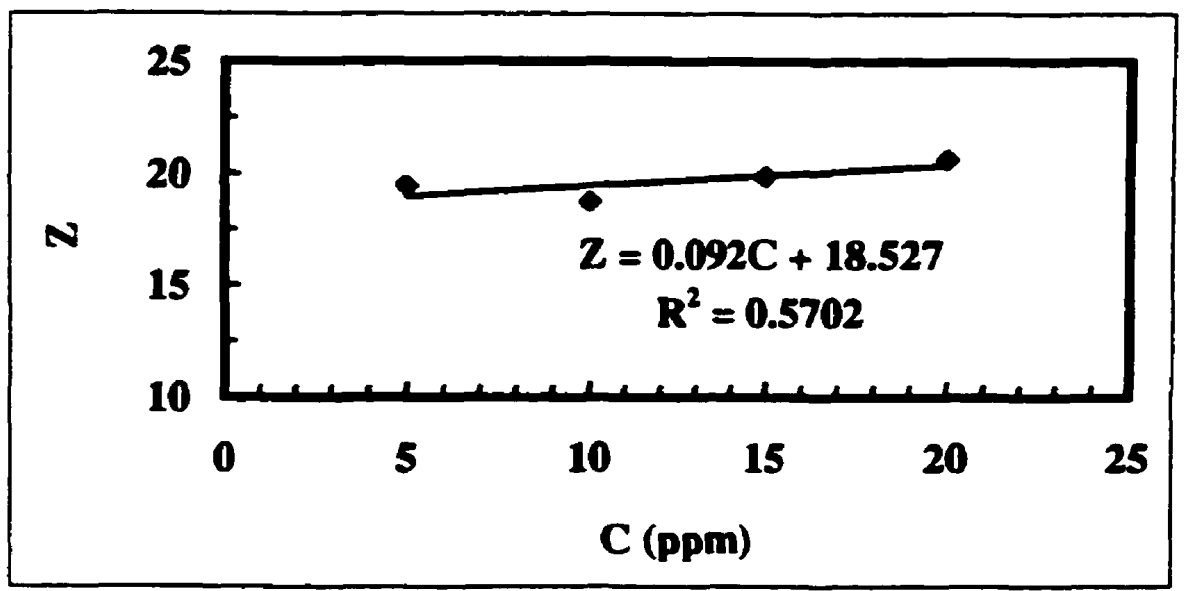


$\frac{I_{\theta}}{I_{(180-\theta)}}=\frac{P(\theta)}{P(180-\theta)}$

$P(\theta)=\left[\left(\frac{3}{x^{3}}\right)(\sin x-x \cos x)\right]^{2}$

$\theta$ (deg.)

$x=2 \pi\left(\frac{D n}{\lambda_{0}}\right) \sin \left(\frac{\theta}{2}\right)$

$\lambda_{0}=514 \mathrm{~nm} \quad \mathrm{n}=1.336$

45

135

$\mathrm{L}_{45} \boldsymbol{I}_{135}$

$\mathrm{z}=$

18.527

$\mathbf{D}=$

$223.8546 \mathrm{~nm}$

Xes

$-1.78014$

$x_{135}$

$-3.65043$

$R_{2}^{2}=\frac{3}{5}\left(\frac{D}{2}\right)^{2}$

$P(45)=$

0.514009

$\mathbf{R g}=$

$86.69851 \mathrm{~nm}$

$\mathbf{P}(\mathbf{1 3 5})=$

0.027744

To find D: use Solver to find a D which

Z-P(45)/P(135)=

0.00 satisfies Z-P(45)/P(135)= 0 .

\section{Reference:}

1. Kratochvil, P., Classical Light Scattering from Polymer Solutions, Elsevier. Amsterdam, 1987. 


\section{Appendix 5.3 Langmuir Kinetics for Microgels}

The objective of this calculation is to attempt to model microgel surface tension kinetics to explain the infuence of crosslinker concentration.

According to van de Ven [1], the original Langmuir kinetic model for polymer adsorption is:

$$
\frac{\mathrm{d} \theta}{\mathrm{dt}}=\mathrm{k}_{1} \cdot\left(\mathrm{n}_{\mathrm{o}}-\theta\right) \cdot(1-\theta)-\mathrm{k}_{2} \cdot \theta \quad \text { Equation A5.3.1 }
$$

$\theta$ is the fraction of the covered surface area;

$k_{1}$ is the adsorption rate constant;

$\mathbf{k}_{2}$ is hte desorption rate constant;

$\mathrm{n}_{\mathbf{o}}$ is the ratio of the initial number of polymer molecules to the maximum number of polymer molecules that can adsorb onto the available surface

By definition,

$$
\theta=\frac{\Gamma}{\Gamma_{\max }}
$$

where $\Gamma$ is the surface concentration of the microgels at the air/water interface in $\mathrm{mg} / \mathrm{m}^{2}, \Gamma_{\max }$ is the maximum capacity of the surface to adsorb polymer in $\mathrm{mg} / \mathrm{m}^{2}$. The mass of a microgel can be calculated using experimental data at $40^{\circ} \mathrm{C}$

$$
m_{\text {gel }}=\frac{4}{3} \pi\left(R_{h 40 C}\right)^{3} \cdot C_{p} \quad \text { Equation A5.3.3 }
$$

where $\mathrm{Cp}$ is the polymer concentration in a microgel. Dong and Hoffman reported water content in crosslinked PNIPAM macrogel as function of temperature [2]. The water content at $40^{\circ} \mathrm{C}$ read from Figure 4 in Reference 2 is 30 wt\%. Assume all the BA crosslinked PNIPAM gels have the same water content after phase separation, ignoring the crosslinker influence, then all the gels have the same polymer concentration within them at $40^{\circ} \mathrm{C}$. Converted from the water content value at $40^{\circ} \mathrm{C}$ : 


$$
C_{p}:=0.7 \cdot \frac{\mathrm{gm}}{\mathrm{cm}^{3}}
$$

Assuming that rigid spheres have cubic packing at the interface, then

$$
\Gamma_{\max }=\frac{\frac{4}{3} \pi\left(R_{h 40 C}\right)^{3} C_{p}}{4 R_{h}{ }^{2}}=\frac{\pi\left(R_{h 40 C}\right)^{3} C_{p}}{3 R_{h}{ }^{2}} \quad \text { Equation A5.3.4 }
$$

where $R_{h}$ is the hydrodynamic radius at $25^{\circ} \mathrm{C}$ in cm (refer to Table 5. 3), if modeling is done at $25^{\circ} \mathrm{C}$. $\mathrm{n}_{\mathrm{o}}$ in Equation A5.3.1 is defined as the ratio of the initial number of polymer molecules to the maximum number of polymer molecules that can adsorb onto the available surface, which is equivalent to

$$
n_{0}=\frac{C_{0} \cdot v}{\Gamma_{\max } \cdot A}=\frac{C_{0} \cdot \varepsilon}{\Gamma_{\max }}
$$

where $C_{0}$ is the initial microgel mass concentration in a suspension in $\mathrm{gm} / \mathrm{cm}^{3}, V$ is the volume of the pendant drop in $\mathrm{cm}^{3}, A$ is the drop surface area in $\mathrm{cm}^{2}$ and $\varepsilon$ is the drop V/A ratio in $\mathrm{cm}$, which is a known experimental parameter.

The following is the calculations of $n_{0}$ values for the four $1 \mathrm{wt} \%$ microgel samples at $25^{\circ} \mathrm{C}$.

For M-1 1 wt \% suspension at $25^{\circ} \mathrm{C}$,

$$
\begin{aligned}
& R_{h 40 C 1}:=1.25 \cdot 10^{-5} \cdot \mathrm{cm} \quad R_{h 1}:=2.71 \cdot 10^{-5} \cdot \mathrm{cm} \\
& \Gamma_{\max 1}:=\frac{\pi\left(R_{h 40 C 1}\right)^{3} C_{p}}{3 R_{h 1}^{2}} \quad \Gamma_{\max 1}=1.949 \cdot 10^{-3} \cdot \frac{\mathrm{mg}}{\mathrm{cm}^{2}} \\
& \Gamma_{\operatorname{maxl}}:=1.949 \cdot 10^{-3} \frac{\mathrm{mg}}{\mathrm{cm}^{2}} \quad C_{0}:=10 \frac{\mathrm{mg}}{\mathrm{cm}^{3}} \quad \varepsilon_{1}:=0.06281 \cdot \mathrm{cm}
\end{aligned}
$$


$\mathrm{n}_{01}:=\frac{C_{0} \cdot \varepsilon_{1}}{\Gamma_{\max 1}} \quad \mathrm{n}_{01}=322.268 \quad\left(\mathrm{n}_{\mathrm{o}}\right.$ is much greater than 1$)$

For M-2 1 wt \% suspension at $25^{\circ} \mathrm{C}$,
$R_{h 40 C 2}:=1.60 \cdot 10^{-5} \cdot \mathrm{cm}$
$R_{h 2}:=2.97 \cdot 10^{-5} \cdot \mathrm{cm}$
$\Gamma_{\max 2}:=\frac{\pi\left(R_{h 40 C 2}\right)^{3} C_{p}}{3 R_{h 2}{ }^{2}}$
$\Gamma_{\max 2}=3.404 \cdot 10^{-3} \cdot \frac{\mathrm{mg}}{\mathrm{cm}^{2}}$
$\Gamma_{\max 2}:=3.404 \cdot 10^{-3} \frac{\mathrm{mg}}{\mathrm{cm}^{2}}$
$C_{0}:=10 \frac{\mathrm{mg}}{\mathrm{cm}^{3}}$
$\varepsilon_{2}:=0.0651 \cdot \mathrm{cm}$
$\mathrm{n}_{02}:=\frac{C_{0} \cdot \varepsilon_{2}}{\Gamma_{\max 2}}$
$\mathrm{n}_{02}=191.246$
( $\mathrm{n}_{\mathrm{o}}$ is Much greater than 1)

For $\mathrm{M}-31 \mathrm{wt} \%$ suspension at $25^{\circ} \mathrm{C}$,

$$
\begin{aligned}
& R_{\mathrm{h} 40 \mathrm{C} 3}:=1.72 \cdot 10^{-5} \mathrm{~cm} \quad R_{\mathrm{h} 3}:=3.25 \cdot 10^{-5} \cdot \mathrm{cm} \\
& \Gamma_{\max 3}:=\frac{\pi\left(R_{h 40 C 3}\right)^{3} C_{p}}{3 R_{h 3}{ }^{2}} \quad \Gamma_{\max 3}=3.531 \cdot 10^{-3} \cdot \frac{\mathrm{mg}}{\mathrm{cm}^{2}} \\
& \Gamma_{\max 3}:=3.531 \cdot 10^{-3} \frac{\mathrm{mg}}{\mathrm{cm}^{2}} \quad C_{0}:=10 \frac{\mathrm{mg}}{\mathrm{cm}^{3}} \quad \varepsilon_{3}:=0.0630 \mathrm{~cm} \\
& \mathrm{n}_{03}:=\frac{C_{0} \cdot \varepsilon_{3}}{\Gamma_{\max 3}} \quad \mathrm{n}_{03}=178.42 \quad\left(\mathrm{n}_{\mathrm{o}} \text { is Much greater than } 1\right)
\end{aligned}
$$

For M-4 1 wt\% suspension at $25^{\circ} \mathrm{C}$,

$$
R_{h 40 C 4}:=1.47 \cdot 10^{-5} \cdot \mathrm{cm} \quad R_{h 4}:=2.89 \cdot 10^{-5} \cdot \mathrm{cm}
$$


$\Gamma_{\max 4}:=\frac{\pi\left(R_{h 40 C 4}\right)^{3} C_{p}}{3 R_{h 4}{ }^{2}} \quad \Gamma_{\max 4}=2.788 \cdot 10^{-3} \cdot \frac{\mathrm{mg}}{\mathrm{cm}^{2}}$

$\Gamma_{\max 4}:=2.788 \cdot 10^{-3} \frac{\mathrm{mg}}{\mathrm{cm}^{2}} \quad C_{0}:=10 \frac{\mathrm{mg}}{\mathrm{cm}^{3}} \quad \varepsilon_{4}:=0.0594 \cdot \mathrm{cm}$

$\mathrm{n}_{04}:=\frac{C_{0} \cdot \varepsilon_{4}}{\Gamma_{\max 4}} \quad n_{04}=213.056 \quad\left(n_{0}\right.$ is Much greater than 1)

$n_{0}$ values of the $41 \mathrm{wt} \%$ microgel samples at $25^{\circ} \mathrm{C}$ are between $1.783^{*} 10^{7}$ and $3.223 * 10^{7}$, which are all much greater than 1 . Therefore $\left(n_{0}-\theta\right)$ in Equation A5.3.1 is approximately equal to $n_{0}$. Further, the maximum coverages $\left(\Gamma_{\max }\right)$ don't vary too much (between $1.9 * 10^{-3}$ and $3.5^{*} 10^{-3} \mathrm{mg} / \mathrm{m}^{2}$ ) and are an order of magnitude higher than Kawaguchi's data for linear polymer (see Appendix 2.5).

If ignoring bulk depletion and assuming no desorption, then Equation A5.3.1 reduced to

$$
\frac{\mathrm{d}}{\mathrm{dt}} \theta=\mathrm{k}_{1} \cdot \mathrm{n}_{\mathrm{o}} \cdot(1-\theta) \quad \text { Equation A5.3.6 }
$$

where $\mathrm{k}_{1}$ has the unit of $\mathrm{s}^{-1}$. The boundary condition for Equation A5.6 is $\boldsymbol{\theta}=0$ when $t=0$. Rearranging Equation A5.3.6,

$$
\frac{d \theta}{1-\theta}=\frac{k_{1} \cdot n_{0}}{d t}
$$

Solving the above equation, it gives

$$
-\ln (1-\theta)=k_{1} \cdot n_{0} \cdot t
$$

Rearranging,

$$
(1-\theta)=\exp \left(-k \cdot n_{0} \cdot t\right) \quad \text { Equation A5.3.7 }
$$


Assume surface tension is a simple function of coverage

$$
\gamma=\gamma_{m}+\left(\gamma_{0}-\gamma_{m}\right) \cdot(1-\theta) \quad \text { Equation A5.3.8 }
$$

where $\gamma_{m}$ is the meso-equilibrium surface tension (see Tabel 5.4) and $\gamma_{0}$ is the surface tension of water, at $25^{\circ} \mathrm{C}$, which is (see Appendix A2.3):

$$
\gamma_{0}:=72.14 \frac{\mathrm{mJ}}{\mathrm{m}^{2}}
$$

Substituting Equation A5.3.7 and Equation A5.3.5 into Equation A5.3.8, the dynamic surface tension is:

$$
\gamma=\gamma_{m}+\left(\gamma_{0}-\gamma_{m}\right) \exp \left[\frac{-3 k_{1} \cdot C_{0} \cdot \varepsilon \cdot R_{h}^{2}}{\pi\left(R_{h 40 C}\right)^{3} \cdot C_{p}} \cdot t\right]
$$

Calculating Surface tension curves. From Table 5.4,

$\gamma_{\mathrm{m} 1}:=42.47 \frac{\mathrm{mJ}}{\mathrm{m}^{2}} \quad \gamma_{\mathrm{m} 2}:=43.48 \frac{\mathrm{mJ}}{\mathrm{m}^{2}} \quad \gamma_{\mathrm{m} 3}:=43.5 \frac{\mathrm{mJ}}{\mathrm{m}^{2}} \quad \gamma_{\mathrm{m} 4}:=45.28 \frac{\mathrm{mJ}}{\mathrm{m}^{2}}$
$\left(\mathbf{M}_{1}\right)$
$\left(\mathbf{M}_{2}\right)$
$\left(\mathbf{M}_{3}\right)$
$\left(\mathbf{M}_{4}\right)$
$t:=s . .8000 \cdot s \quad k_{1}:=0.000163 \cdot s^{-1}$

$k_{1}$ is the adsorption rate constant - this is the only adjustable parameter roughly choosen so the predictions for $M-1$ and $M-2$ curves are approximately correct. $\gamma\left(t, \gamma_{m}, R_{h}, R_{h 40 C}, \varepsilon\right):=\gamma_{m}+\left(\gamma_{0}-\gamma_{m}\right) \exp \left[\frac{-3 k_{1} \cdot C_{0} \cdot \varepsilon \cdot R_{h}{ }^{2}}{\pi\left(R_{h 40 C}\right)^{3} \cdot C_{p}}\right]$ 


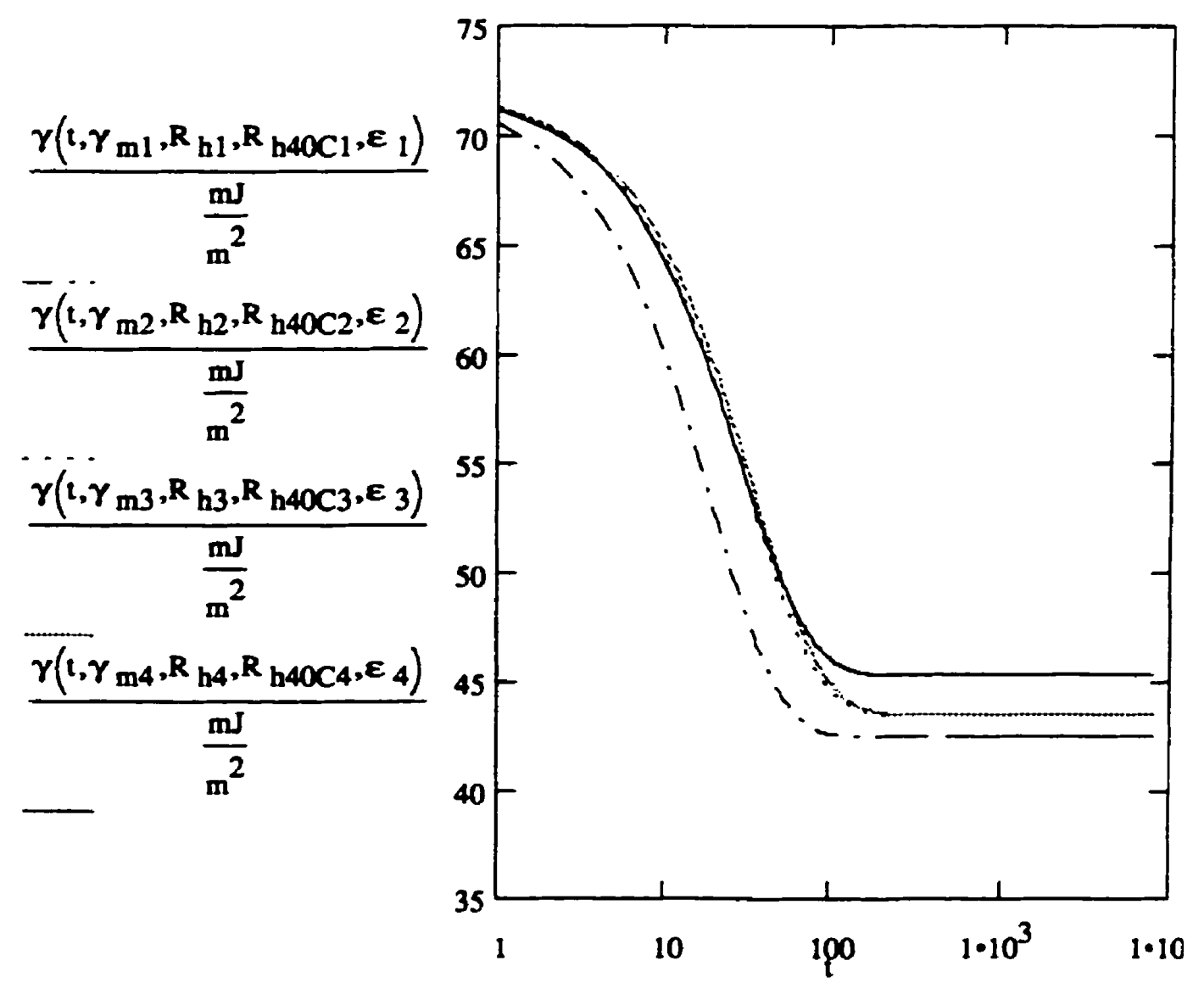

\section{Reference:}

1. van de Ven, T.G.M., Advances Colloid Interface Sci., 48, 121 (1994).

2. Dong, L. C. and Hoffman, A. S., J. Controlled Release, 13, 21 (1990). 


\section{Appendix 5.4 Approximation of the Meso-equilibrium Surface Concentration of $\mathbf{N}$-isopropyl Groups}

The objective of this calculation is to approximate the meso-equilibrium surface concentration of $\mathbf{N}$-isopropyl groups at the air/water interface, using the meso-equilibrium surface tensions of the microgel suspensions and the empirical relationship between the surface tension and the surface concentration of linear NIPAM homopolymers (see Appendix 2.5).

According to Equation A2.5.11 in Appendix 2.5, the surface concentration of NIPAM at $25^{\circ} \mathrm{C}\left(\Gamma_{\text {NIPAM2SC }}\right)$ as a function of the surface tension $(\gamma)$ is:

$$
r_{\text {NIPAM25C }}(\gamma)=\left(0.6002+0.0459 \cdot \operatorname{Ln}\left(\frac{72.14-\gamma}{\gamma-42.19}\right)\right) \cdot \frac{\mathrm{mg}}{\mathrm{m}^{2}} \quad \text { Equation A5.4.1 }
$$

The weight percentage of the $\mathrm{N}$-isopropyl group in a $\mathrm{N}$-isopropylacrylamide molecule is:

$$
\begin{aligned}
& \text { NIPAM }_{\text {IP\% }}=\frac{3 C+7 \mathrm{H}}{6 \mathrm{C}+11 \mathrm{H}+\mathrm{O}+\mathrm{N}} \\
& \text { NIPAM }_{\text {IP\% }}:=\frac{3 \cdot 12.011+7 \cdot 1.0079}{6 \cdot 12.011+11 \cdot 1.0079+15.9994+14.0067} \\
& \text { NIPAM }_{I P \%}=38.078 \% \%
\end{aligned}
$$

Multiplying both sides of Equation A5.4.1 with NIPAMP\%, it gives the surface concentration of $\mathrm{N}$-isopropyl groups at $25^{\circ} \mathrm{C}\left(\Gamma_{\text {IP2SC }}\right)$ as:

$$
r_{\text {IP2SC }}=\left(0.2285+0.0175 \cdot \operatorname{Ln}\left(\frac{72.14-\gamma}{\gamma-42.19}\right)\right) \frac{\mathrm{mg}}{\mathrm{m}^{2}} \quad \text { Equation A5.4.2 }
$$

From Table 5.4, the meso-equilibrium surface tension $\left(\gamma_{\mathrm{mi}}, \mathrm{i}=1\right.$ to 4 , denoting M-1 to M-4 respectively) for the four $1 \mathrm{wt} \%$ microgel sispensions at $25^{\circ} \mathrm{C}$ are :

$$
\gamma_{\mathrm{ml}}:=42.47 \frac{\mathrm{mJ}}{\mathrm{m}^{2}} \quad \gamma_{\mathrm{m} 2}:=43.48 \frac{\mathrm{mJ}}{\mathrm{m}^{2}} \quad \gamma_{\mathrm{m} 3}:=43.5 \frac{\mathrm{mJ}}{\mathrm{m}^{2}} \quad \gamma_{\mathrm{m} 4}:=45.28 \frac{\mathrm{mJ}}{\mathrm{m}^{2}}
$$


The meso-equilibrium surface concentrations of the $\mathbf{N}$-isopropyl groups at the air/water interface are approximated as:

\section{M-1}

$\Gamma_{\text {IP2SCl }}:=\left(0.2285+0.0175 \cdot \ln \left(\frac{72.14-\gamma_{\mathrm{ml}}}{\gamma_{\mathrm{ml}}-42.19}\right)\right) \cdot \frac{\mathrm{mg}}{\mathrm{m}^{2}} \quad \Gamma_{\mathrm{IP25C1}}=0.31 \cdot \frac{\mathrm{mg}}{\mathrm{m}^{2}}$

\section{M-2}

$\Gamma_{\text {IP2SC2 }}:=\left(0.2285+0.0175 \cdot \ln \left(\frac{72.14-\gamma_{\mathrm{m} 2}}{\gamma_{\mathrm{m} 2}-42.19}\right)\right) \frac{\mathrm{mg}}{\mathrm{m}^{2}} \quad \Gamma_{\text {IP25C2 }}=0.283 \cdot \frac{\mathrm{mg}}{\mathrm{m}^{2}}$

M-3

$\Gamma_{\text {IP25C3 }}:=\left(0.2285+0.0175 \cdot \ln \left(\frac{72.14-\gamma_{\mathrm{m} 3}}{\gamma_{\mathrm{m} 3}-42.19}\right)\right) \cdot \frac{\mathrm{mg}}{\mathrm{m}^{2}} \quad \Gamma_{\text {IP25C3 }}=0.282 \cdot \frac{\mathrm{mg}}{\mathrm{m}^{2}}$

M-4

$\Gamma_{\text {IP25C4 }}:=\left(0.2285+0.0175 \cdot \ln \left(\frac{72.14-\gamma_{\mathrm{m} 4}}{\gamma_{\mathrm{m} 4}-42.19}\right)\right) \frac{\mathrm{mg}_{\mathrm{g}}}{\mathrm{m}^{2}} \quad \Gamma_{\text {IP25C4 }}=0.266 \cdot \frac{\mathrm{mg}}{\mathrm{m}^{2}}$

And for 0.05 wt $\%$ M-1 suspension at $25^{\circ} \mathrm{C}, \gamma_{\mathrm{m} 10.05 \%}:=44.96 \frac{\mathrm{mJ}}{\mathrm{m}^{2}}$

$\Gamma_{\text {IP2SC10.05\% }}:=\left(0.2285+0.0175 \cdot \ln \left(\frac{72.14-\gamma_{\mathrm{m} 10.05 \%}}{\gamma_{\mathrm{m} 10.05 \%}-42.19}\right)\right) \frac{\mathrm{mg}}{\mathrm{m}^{2}}$

$\Gamma_{\text {IP2SC10.05\% }}=0.268 \cdot \frac{\mathrm{mg}}{\mathrm{m}^{2}}$ 


\title{
Chapter $6^{*}$
}

\section{The Temperature Dependent Contact Angles of Water on Poly( $N$-isopropylacrylamide) Gels}

\begin{abstract}
The advancing contact angles of water on cross-linked poly $(N$ isopropylacrylamide) water swollen gels were a sensitive function of temperature. From 25 to $35{ }^{\circ} \mathrm{C}$ the angles were close to $40^{\circ}$ whereas from 35 to $50^{\circ} \mathrm{C}$ the angles were about $90^{\circ}$. The abrupt change in wetting corresponded to the temperature range in which the gel shrank from about 90 to $20 \mathrm{wt} \%$ water upon heating. It is speculated that the iso-propyl groups preferentially occupied the air water interface.
\end{abstract}

- Reprinted in part with permission from Langmuir 11. 2301-2302 (1995). Copyright 1995 American Chemical Society. 


\subsection{Introduction}

This communication describes results of the first reported contact angle measurements on water swollen cross-linked poly( $N$-isopropylacrylamide) (PNIPAM) macrogels which show unique behavior as a function of temperature. Many papers in the last decade have discussed the swelling properties of crosslinked PNIPAM gels in water [1]. This is an interesting system because the gels are highly swollen (typically $90 \%$ by weight of water) up to $32{ }^{\circ} \mathrm{C}$, whereas at higher temperatures the gels expel most of the water to leave about 2 water molecules per amide unit. Temperature sensitive swelling has been observed in both macroscopic [2] and microscopic colloidal microgels [3] and the volume phase transition temperature (i.e. the temperature corresponding to the greatest change in volume), $\mathrm{Tv}$, is in the range $31-35^{\circ} \mathrm{C}$. This behavior reflects the fact that linear PNIPAM has a lower critical solution temperature in water at about 32 ${ }^{\circ} \mathrm{C}[4]$.

Figure 1 shows the advancing contact angle of water as a function of temperature. The behavior was remarkable. At $25{ }^{\circ} \mathrm{C}$ when the gel contained about $90 \%$ water, the contact angle was $42^{\circ}$. Around the $\operatorname{Tv}$ (about $36^{\circ} \mathrm{C}$ ) the contact angle jumped to $90^{\circ}$, which corresponds to values for water on alkane surfaces. Very recently Takei et al. have reported similar temperature dependent contact angles for PNIPAM grafted onto glass [5]. Similarly, Yamada et al. reported a contact angle of $48^{\circ}$ for water on PNIPAM treated glass at $37^{\circ} \mathrm{C}$ [6]. 


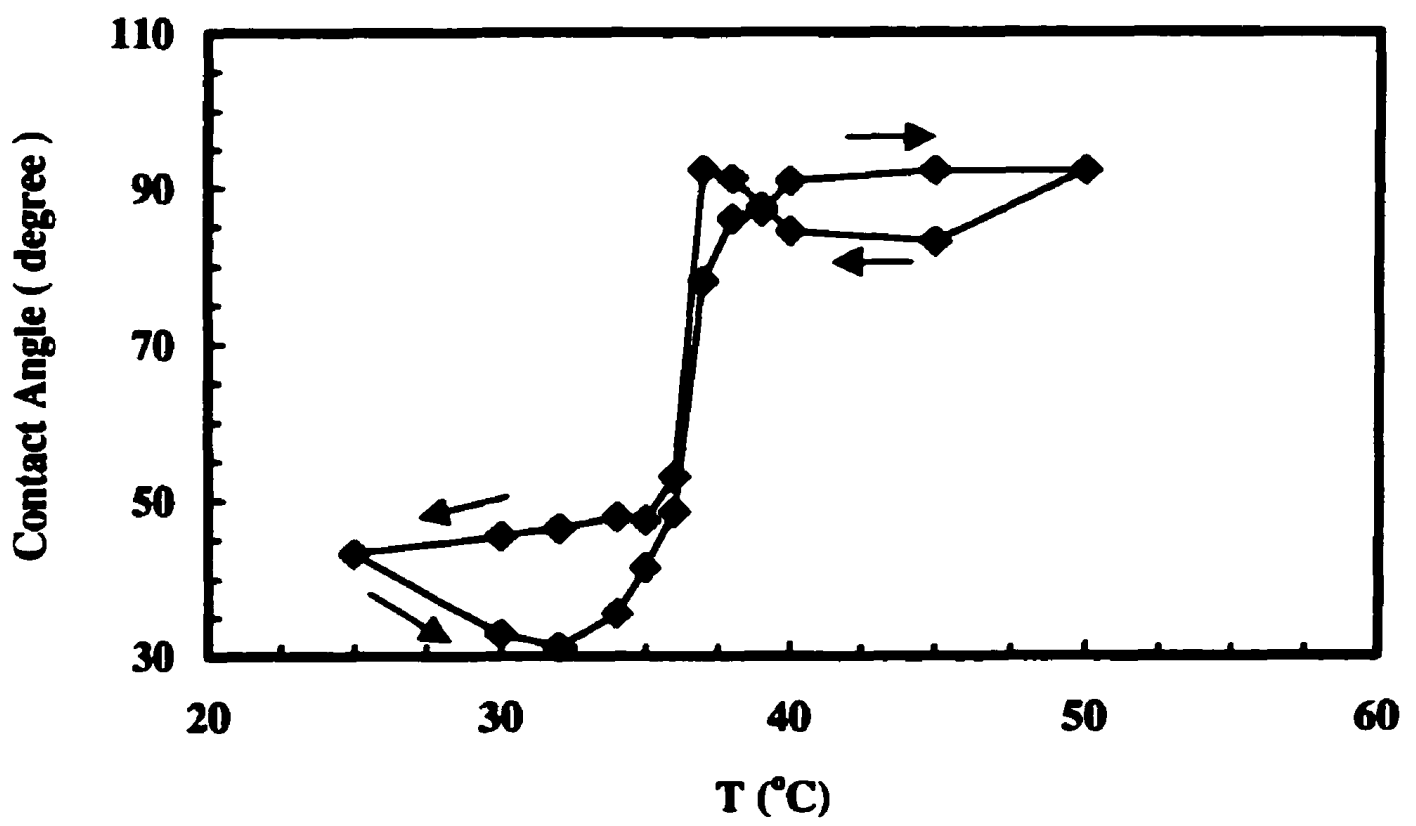

Figure 1 The advancing contact angle of water on cross-linked PNIPAM gel swollen with water. The initial measurement was made at $25^{\circ} \mathrm{C}$ and the arrows indicate the direction of temperature change.

The arrows in Figure 1 show the direction in which the temperature was changed. Although a minimum of $\mathbf{3 0}$ minutes was given for equilibration, we believe the scatter reflects incomplete swelling or de-swelling of the gel. A slow de-swelling kinetics has been reported by others [7].

The results can most obviously be interpreted by assuming that isopropyl groups tend to concentrate at the air/water interface at all temperatures. At high temperatures the result is an alkane-like surface of packed isopropyl groups whereas at low temperature there are some water and amide groups mixed in at the interface. 
Attempts to measure a contact angle by the captive bubble method with the gel immersed in water were unsuccessful. Below Tv, the bubbles did not adhere indicating a contact angle of zero. At higher temperatures the results were erratic; in some cases the captive bubble would adhere to give a contact angle whereas in repeated measurement at the same location the air bubble did not displace water from the surface. Perhaps with immersed gels the more polar amide groups were facing the water phase. The concept of polymers and gels orienting to give the minimum interfacial tension has been proposed by others [8].

High contact angles for water on other types of hydrogels has been reported. For example, Holly and Refojo reported advancing contact angles between 60 and $85^{\circ}$ for cross-linked poly(2-hydroxyethyl methacrylate) swollen gels containing 30 to $40 \%$ water [9]. Similarly, Chappuis et al showed that saline solution had an advancing contact angle of $100^{\circ}$ on animal cartilage [10]. Therefore, we conclude that the PNIPAM results are reasonable.

The swelling behavior of PNIPAM gels is influenced by solvent composition, surfactant and the presence of acrylamide or other co-monomers. We speculate that the contact angle will also be sensitive to these factors and work is in progress to verify this.

In summary, we believe the results in Figure 1 are the first reported contact angles for water on PNIPAM gels and we do not know of any other system where the contact angles are such a strong function of temperature. 


\subsection{Experimental}

NIPAM (Kodak) was purified by dissolution in toluene and recrystallization with n-hexane. Methylenebisacrylamide (Aldrich), potassium persulfate (BDH, analytic grade), and sodium bisulfate (BDH, laboratory reagent), were used as received. Milli Q water (Millipore Corp.) was used for all solutions.

To a $100 \times 50$ crystallizing dish (Pyrex) was added $4 \mathrm{~g}$ NIPAM and $0.1 \mathrm{~g}$ methylenebisacrylamide in $\mathbf{4 0} \mathrm{mL}$ water. The dish was sealed with a rubber stopper and oxygen was removed from the mixture by nitrogen bubbling. With the temperature controlled at $18{ }^{\circ} \mathrm{C}$, the mixture was stirred with a magnetic bar and polymerization was initiated by the addition of $5 \mathrm{mg}$ sodium bisulfite and 5 mg potassium persulfate. After 10 minutes the stirring and nitrogen flow were stopped and the system was sealed. After 2 hours the transparent, elastic crosslinked polyNIPAM gel was removed and extensively washed in water. The cleaned gel was cut into $12 \times 4 \times 2 \mathrm{~mm}$ samples, which were stored in water.

Advancing contact angles were measured by the sessile drop method with a Rame-Hart Model 100-00 Contact Angle Goniometer fitted with a Model 10007 Environmental Chamber. Gel samples were positioned with the side cast against the crystallizing dish facing upwards, maintaining water around the substrate base to keep the vapor phase saturated. The chamber was closed and 
conditioned with a Brinkman Model RMS- 6 water bath set at $25{ }^{\circ} \mathrm{C}$ for about $\mathrm{lh}$, after which the chamber was opened and any excess water on top of the gel was blotted with filter paper. Ten minutes later, a $0.5 \mu \mathrm{L}$ sessile drop of water was placed on the gel and the advancing contact angle was immediately measured when the three-phase boundary of the drop stopped moving. Angles on both sides of each drop were measured to assure symmetry. The gel was allowed $\mathbf{3 0}$ minutes to equilibrate after each temperature change. Two batches of gel were polymerized and both showed the same contact angle behavior.

We recognize that contact angle measurements on gels are subject to difficulties arising from the deformation of the gel [11]. However, the changes in contact angle with temperature observed in this work were sufficiently high that small errors due to deformation were unlikely to influence the conclusions. 


\subsection{Acknowledgments}

This work wàs partially supported by the Canadian Natural Science and Engineering Research Council. The authors also acknowledge the Provincial Government of Shandong Province of the People's Republic of China for financial support of Ju Zhang. 


\subsection{References}

1 Schild, H.G., Prog. Polym. Sci., 17, 163 (1992).

2 Dong, L.C. and Hoffman, A.S., J. Controlled Release, 13, 21, 1990.

3 Pelton, R.H., Pelton, H.M., Morfesis, A. and Rowell, R.L., Langmuir, 5, 816, 1989

4 Heskin, M. and Guillet, J.E., J. Macromol. Sci.-Chem., A2(8), 1441, 1968.

5 Takei, Y.G., Aoki, T., Sanui, K., Ogata, N., Sakurai, Y., and Okano, Y., Macromolecules, 27, 6163 (1994).

6 Yamada, N., Okano, T., Sakai, H., Karikusa, F., Sawasaki, U., and Sakurai, Y., Makromolek. Chem., Rapid Commun. 11, 571 (1990).

7 Chiklis, C.K. and Grasshoff, J.M., J. Polym. Sci., A-2, 8, 1617 (1970).

8 Yasuda, H. Sharma, A.K. and Yasuda, T., J Polym. Sci., Polym. Physics Edition, 19,1285 (1981).

9 Holly, F.J. and Refojo, M.F., J. Biomed. Res., 9, 315(1975).

10 Chappuis, J., Sherman, I.A., and Neumann, A. W., Annals of Biomed. Eng., 11, 435 (1985).

11 Andrade, J.D. and King, R.N., J. Polymer Sci., Polymer Symposium, 66, 313 (1979). 


\section{Chapter 7 Conclusion}

\subsection{Conclusion on NIPAM Polymers at the Air/Water Interface}

For the first time for any material, the interfacial properties at the air/water interface of linear homopolymers, copolymers and crosslinked gels have been characterized. Furthermore, the temperature sensitivity of NIPAM-based materials has enabled the study of the interfacial properties as a function of the polymer/water affinity.

The dynamics of surface layer formation is an important aspect of this thesis. Before the phase separation, the random coils of linear NIPAM homopolymer diffuse to the air/water interface and then the polymer chains unwrap and spread out to cover the air/water interface and lower the surface tension of water. Although there is not direct evidence, it seems reasonable to assume that the hydrophobic $N$-isopropyl groups preferentially protrude into the

air. The proposed sequence of events during adsorption is shown in Figure 7. 1 (similar to Figure 5.7, should be viewed from the bottom up). 

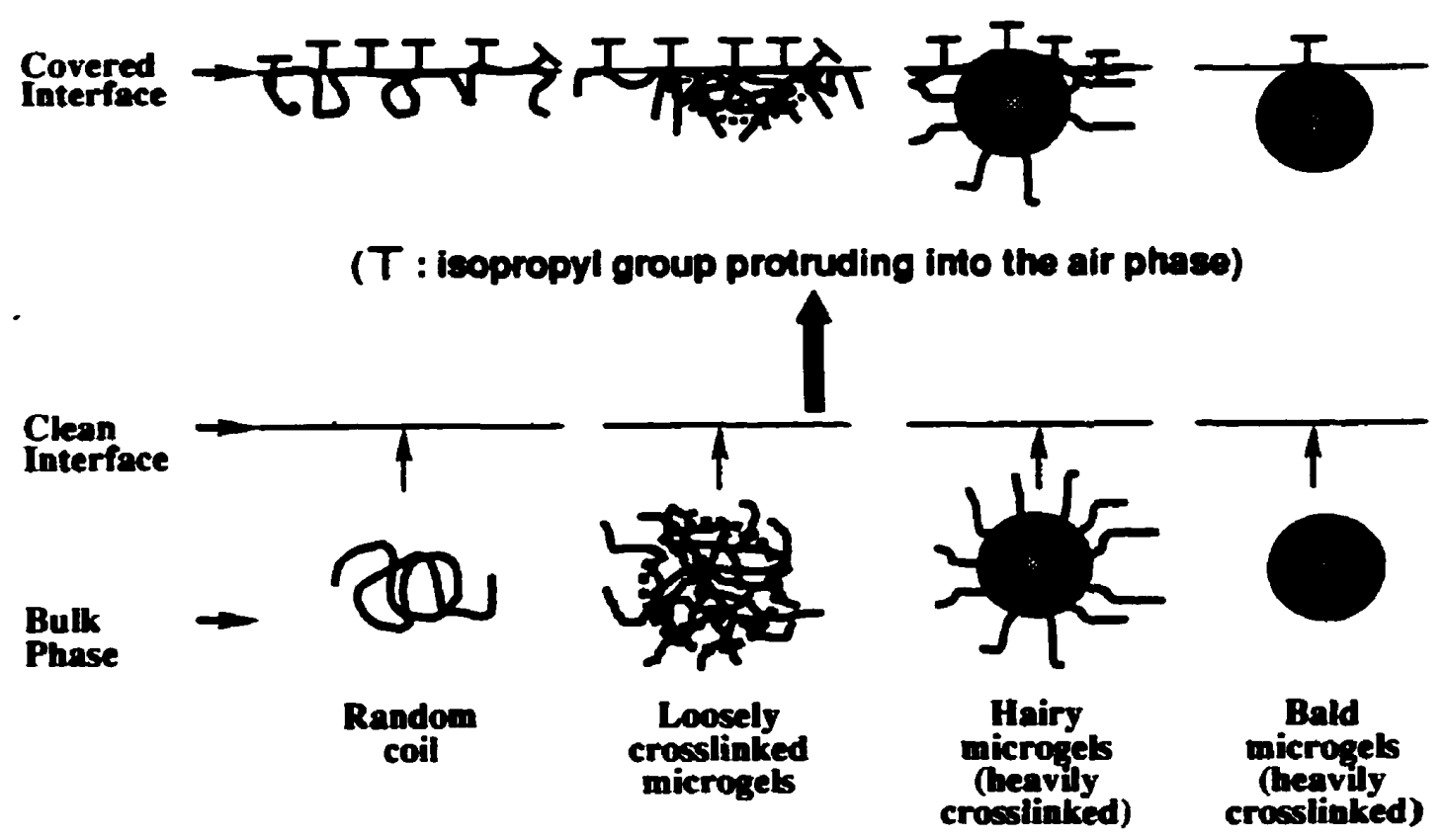

Figure 7.1 A proposed picture showing how NIPAM polymers lower the surface tension of water before the phase separation of linear polymers or volume phase transition of the microgels.

Surprisingly, the equilibrium surface tension of the various NIPAM-based materials was not very temperature sensitive - this will be discussed later. On the other hand, the dynamics of surface tension lowering was much slower above the phase separation temperature. Above the LCST, phase-separated linear NIPAM homopolymers are present as colloidally stable particles which slowly diffuse to the interface and slowly unwrap. This behavior is illustrated in Figure 7. 2. After arrival at the air/water interface, dehydrated linear polymers chains released from the particles, unwrap, spread to occupy the interface and lower the surface tension of water, and again with the hydrophobic $N$-isopropyl groups preferably protruding into the air phase. 

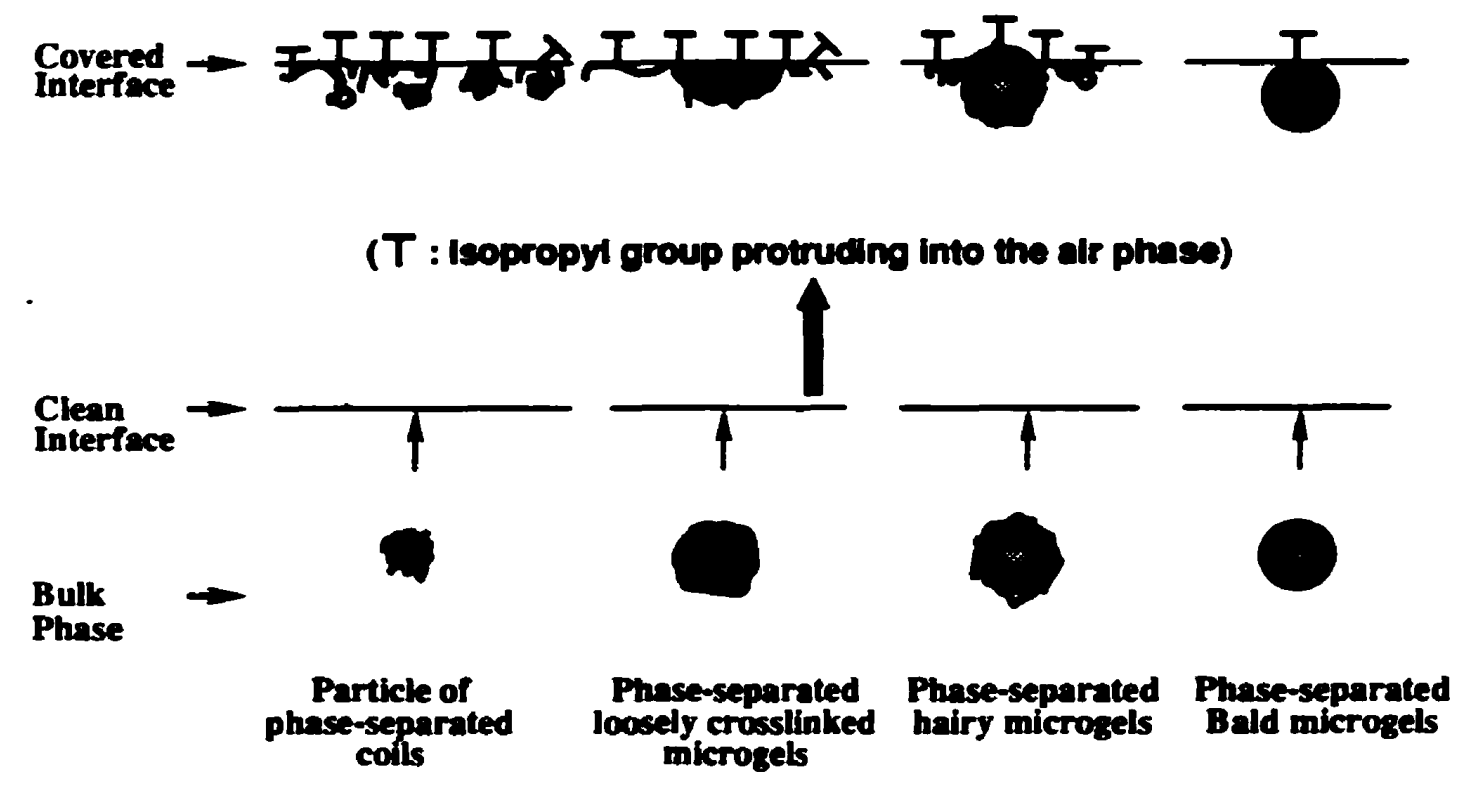

Figure 7.2 A proposed picture showing how NIPAM polymers lower the surface tension of water after the phase separation of linear polymers or volume phase transition of the microgels.

The meso-equilibrium surface tension of an aqueous solution of linear NIPAM homopolymers is not a sensitive function of temperature, whereas other properties in an aqueous bulk phase are. It is inferred that PNIPAM does not phase separate at the air/water interface. It is generally accepted that both the phase separation or volume phase transition are driven by the entropy gain through the release of water molecules from the cage-like structures around the $N$ isopropyl group. Hydrogen bonding and hydrophobic interactions between PNIPAM and water are the major interactions and the source of thermal reversible sensitivity. Because the air phase is hydrophobic, the hydrophobic $\boldsymbol{N}$-isopropyl groups preferably orient into the air phase with fewer, if any, associated water 
molecules. Therefore without bound water, there is no driving force for phase separation (see Figure 7. 3 for symbolic view).

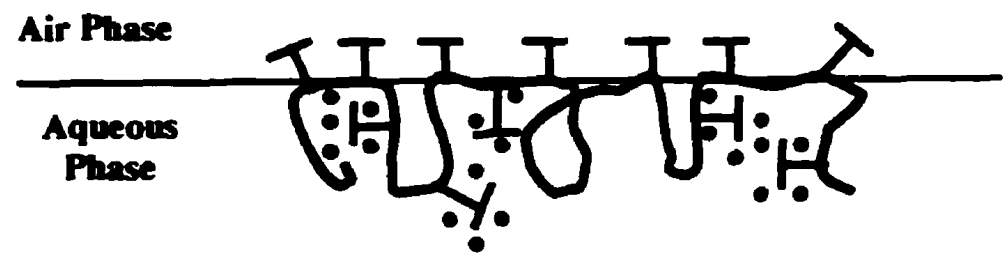

- Water molecule in cage-like structures

T N-isopropyl group

Figure 7.3 Hydrophobic $N$-isopropyl groups in an aqueous phase have associated water molecules whereas it is proposed that surface $N$-isopropyl groups do not.

Surface tension lowering kinetics is a sensitive function of the aqueous phase concentration. The time required for dynamic surface tension to approach its meso-equilibrium value decreases with increasing aqueous phase concentration (see Figures 3.2, 3.3 and 3.4), which is not only observed by the experimental observation, but also is predicted by the simple mass transport models discussed in Chapter 3. However, the simple mass transport models can not predict the time scale for surface tension lowering over the range of polymer concentrations. It is proposed that a slow process involving the penetration of polymers through previously adsorbed polymers contributes to the kinetics. 


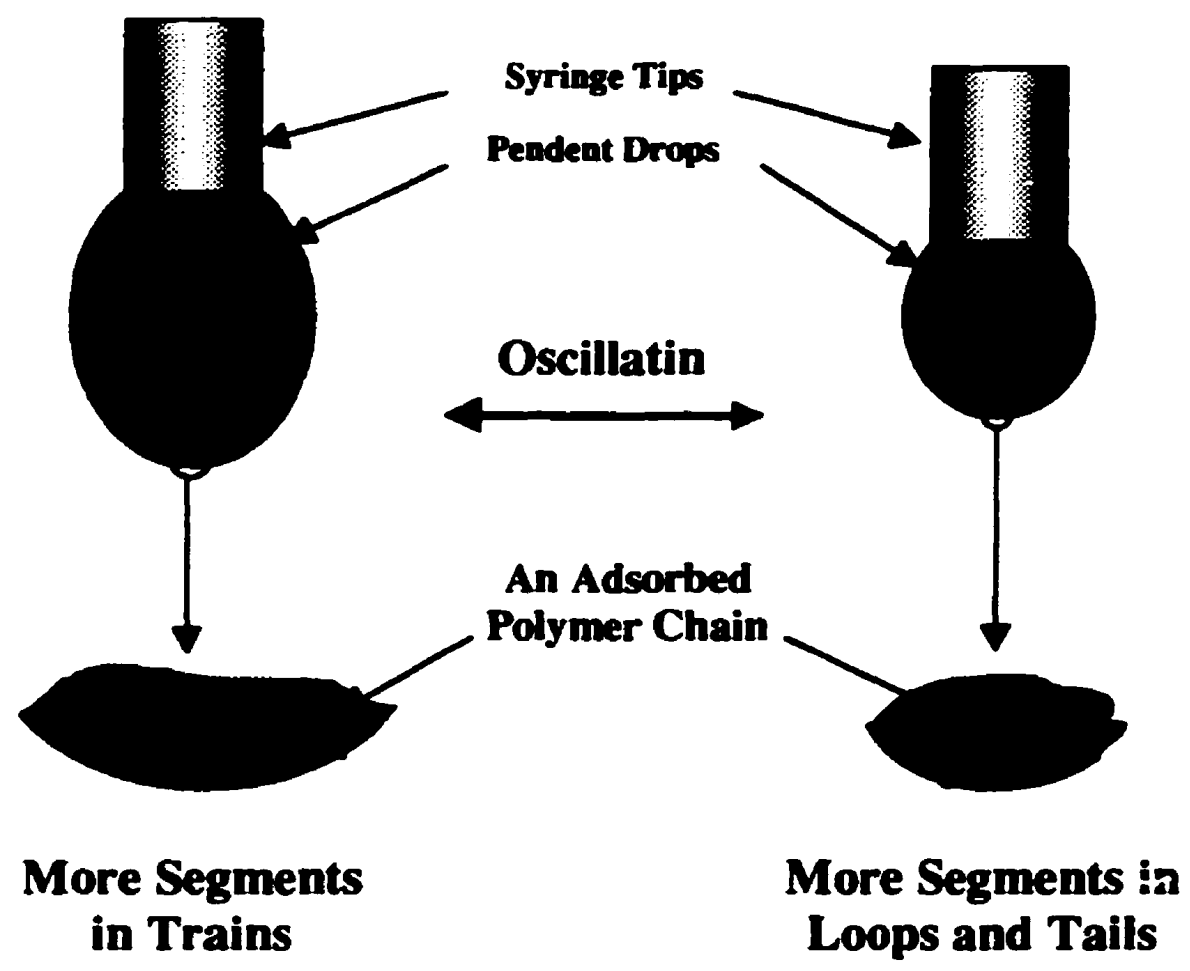

Figure 7.4 A symbolic picture describing the proposed re-arranging of an adsorbed linear NIPAM polymer chain at the oscillating air/water interface in an oscillating pendent drop experiment.

The detailed structure of the adsorbed layer is illusive, however, the novel oscillating drop experiments did provide new information about surface relaxation processes. Oscillations of the pendent drop surface area induce a small surface tension oscillation at long times. The amplitude is a function of the extent of surface dilation, which reflects the ability of loops to become trains when the surface expands. A two-parameter model for non-spreading disks predicts the major features of the oscillating pendent drop experiments on NIPAM homopolymer. However, the experimental steady-state surface tensions oscillations are less than the model prediction, indicating the adsorbed polymer 
floating at the air/water interface can re-arrange to compensate for a changing interface. It is proposed that when the air/water interface dilates, an adsorbed linear NIPAM polymer loops can flatten on the surface to become trains. When the interface contracts, the adsorbed polymer chain is compressed and some of the adsorbed segments present as trains, desorb to become loops or tails - this behavior is illustrated in Figure 7. 4.

The incorporation of acrylamide moieties decreased the interfacial activity of PNIPAM. This behavior was expected because polyacrylamide is very hydrophilic and not surface-active. On the other hand, the surface tension was not a linear function of the acrylamide content. Instead, a novel one-parameter model has been proposed which predicts the experimental results with very good precision.

All the kinetic evidence suggests that the dynamics of poly(NIPAM-coAM) copolymer adsorption is similar to that of NIPAM homopolymer adsorption (see Figure 7. 1 and Figure 7. 2, random coil and particles of phase-separated coils for reference). The difference to the NIPAM homopolymers is that not all the monomer units of a copolymer chain arriving at the interface are surfaceactive. The polymerized NIPAM units are the source of the surface-active property of the copolymers. It is inferred that the polymerized NIPAM units are more enriched at the air phase side of the interphase and the polymerized AM units are more concentrated at the aqueous side of the interphase. Figure 7. 5 
serves as an illustration of the preferential partition of polymerized NIPAM and AM at the air/water interface.

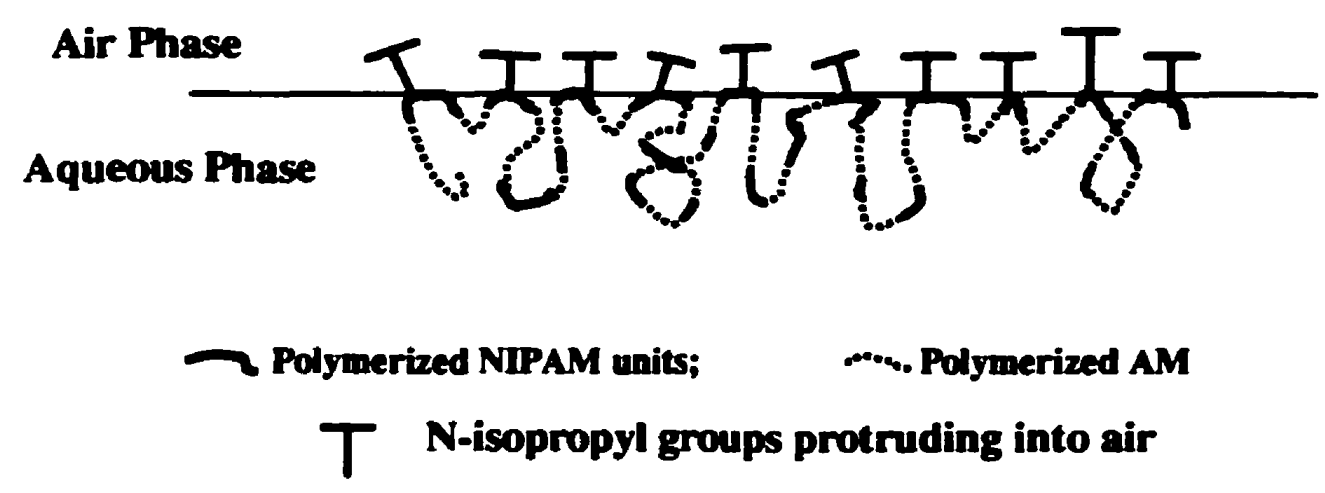

Figure 7.5 An illustration of the preferential partition of the polymerized NIPAM and AM units.

It is not clear how polymerized AM units interfere with the surface-active behavior of the polymerized NIPAM units. It is known that heats of phase separation for the copolymer were less than expected compared with NIPAM homopolymers. It was proposed that NIPAM moieties directly bonded to acrylamide did not contribute to the enthalpy of phase separation.

For the first time, crosslinked microgels are found to lower the surface tension of water. Again, shown in Figure 7.1 and Figure 7. 2, similar to the case of linear NIPAM polymers, crosslinked NIPAM microgels should first diffuse to the air/water interface. However, the crosslinks limit the extent to which microgels can unwrap and spread on the interface. Figure 7.6 illustrates the range of structures prepared in this work. The proposed interfacial behaviors of 
these structures are illustrated in Figure 7.1 and Figure 7. 2. After arriving at the air/water interface, crosslinked NIPAM microgels more or less deform, depending on the extent of crosslinking, and the dangling NIPAM homopolymer chains unwrap and spread out in the process of lowering the surface tension of water. ESEM images showing an ordered array of about $500 \mathrm{~nm}$ diameter spheres of heavily crosslinked NIPAM microgels adsorbed at the air/water interface, were one case of direct evidence. As expected we were unable to obtain ESEM images for loosely crosslinked gels which were able to spread upon the interface.

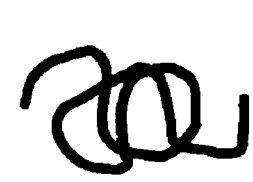

Random coil

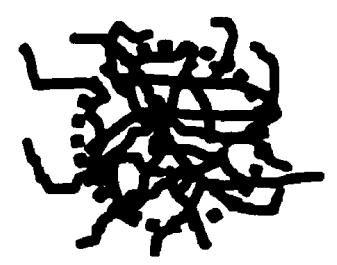

Loosely crooslinked microgel

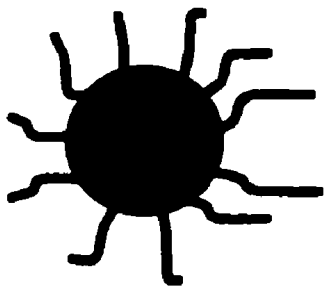

Heavily crosslinked hairy microgel

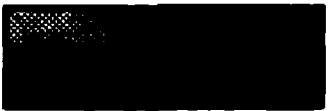

Crosslinked macrogel

\section{Polymer chain mobility decreases \\ Crosslinking degree increases}

Figure 7.6 An illustration of the decrease of polymer chain mobility with the increase of crosslinking degree.

The one parameter Langmuir model derived in Chapter 5 gives the surface tension lowering ability order as $\mathbf{M}-1>\mathbf{M}-2>\mathbf{M}-4>\mathbf{M}-3$. This predicted order agrees with the surface tension lowering order concluded from the experimental results. Both the predicted and experimental orders of surface 
tension lowering are consistent with the order of $R_{h}$ values, $M-1<M-2<M-4<M-3$. The fact that the smaller the hydrodynamic radius of a microgel the faster the surface tension lowering process indicates that diffusion of the microgels to the air/water interface influence the surface tension process. While the prediction of the surface tension lowering order is correct, the model was not successful at predicting the time scale for the dynamic surface tension change. Using only one adjustable parameter $\left(k_{1}\right)$, the model can only fit well to the experimental curve of M-2. The failure of the model may be because of over-simplification of the actual situation by assuming rigid microgel spheres and ignoring the contribution of dangling PNIPAM chains in lowering surface tension. All the polymer chains in a water-swollen microgel are very mobile. However, chains restricted by crosslinks can not as easily spread out to occupy bare air/water interface as linear NIPAM homopolymer chains can. In such a way, crosslinking strongly influenced the mobility of the polymer chains in the microgels. The microgels are not rigid, although crosslinking affects the polymer chain mobility. And the dangling chains, which are more mobile than the chains between crosslinks, can spread out at the air/water interface and contribute to the surface tension lowering.

It is proposed that less crosslinked NIPAM polymers occupy the air water interface more efficiently because of higher polymer chain mobility. For example, among all the hydrated or dehydrated NIPAM polymers, NIPAM homopolymer coils before phase separation has the smallest hydrodynamic radius 
in the order of $10^{-2} \mathrm{~nm}$ and the greatest polymer chain mobility. As a result, aqueous linear NIPAM homopolymers before the phase separation have the fastest surface tension lowering (see Figure 5.4, Figure 5.5 and Table 2.2 for example).

Finally, the contact angle of water on water-swollen macrogel is temperature sensitive. From 25 to $35^{\circ} \mathrm{C}$ the angles were close to $40^{\circ}$ whereas from 35 to $50^{\circ} \mathrm{C}$ the angles were about $90^{\circ}$. Contact angle hysteresis is observed between heating and cooling curves (see Figure 6.1 for reference). The abrupt change in wetting corresponded to the temperature range in which the gel shrinks from about 90 to $20 \mathrm{wt} \%$ water upon heating. The non-zero contact angle of water on the gel surface indicates that macroscopic water swollen gels like the air/water interface covered by linear polymers and the microgels displayed a low surface energy due to the accumulation of $\boldsymbol{N}$-isopropyl groups at the interface. However, unlike the microgels and linear polymers, the surface characteristics reflected in contact angle measurements was sensitive to temperature in the critical range 35 to $40^{\circ} \mathrm{C}$. Figure 7.7 is good only for illustration that at high temperatures, the surface of a deswollen macrogel behaves as an alkane-like surface of packed $N$-isopropyl groups, whereas at low temperature, a waterswollen gel has a surface of the isopropyl group substituted amide groups mixed with water. The contact angle behavior indicates that at all the temperatures, the 
$N$-isopropyl groups preferentially occupied the air/water interface and protruding into the air phase even when the polymer backbone is highly crosslinked.

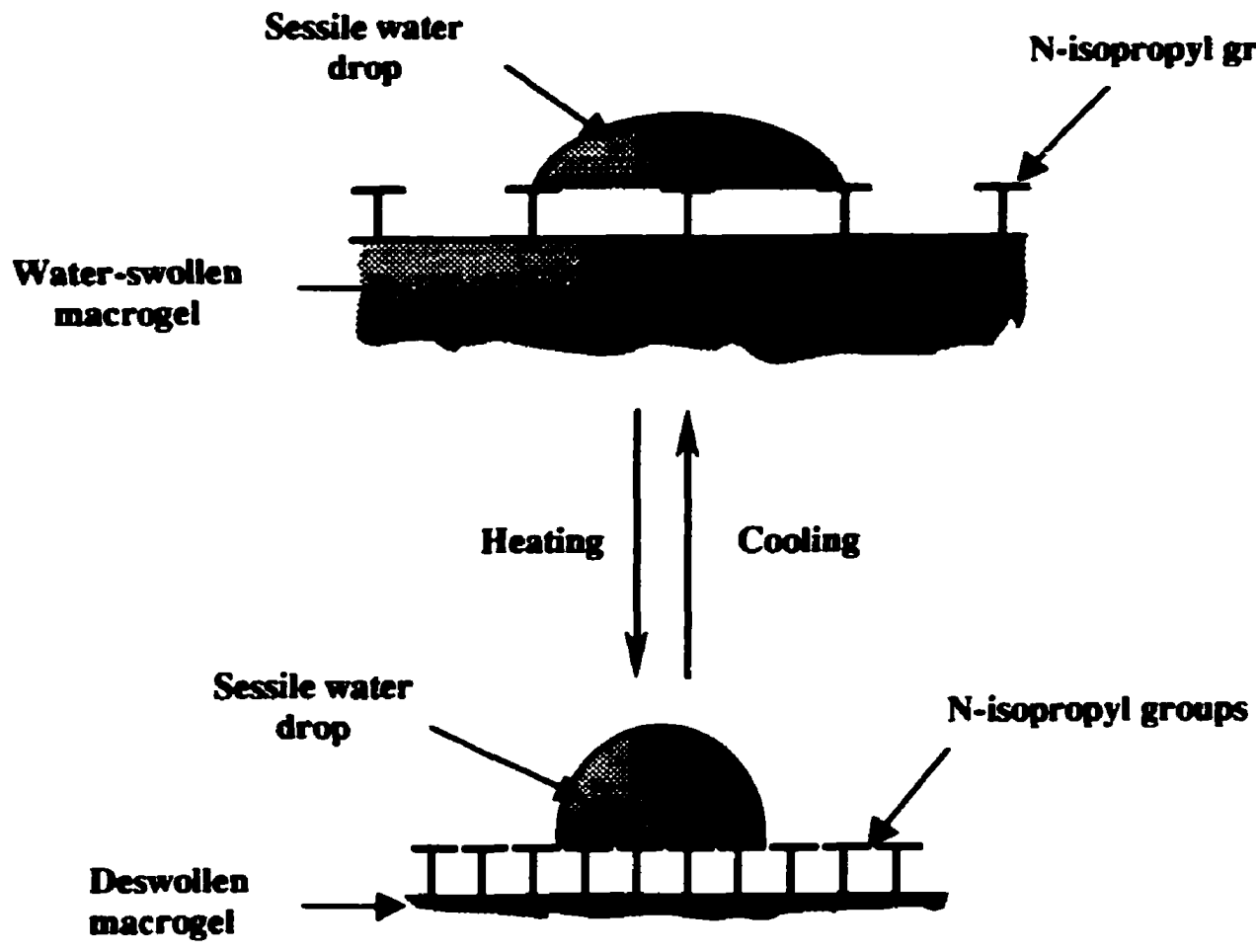

Figure 7. 7 An illustration of the contact angle behavior before and after the volume phase transition of crosslinked NIPAM macrogels.

In summary, NIPAM polymers are surface-active at the air/water interface. Linear NIPAM polymers and crosslinked NIPAM microgels can lower the surface tension of water both before and after the phase separation of volume transition. Meso-equilibrium surface tension of an aqueous solution of linear NIPAM polymers or a suspension of crosslinked microgels is not very 
temperature sensitive, whereas, contact angle of water on water-swollen crosslinked macrogels is temperature dependent. Further, adsorbed NIPAM homopolymer can re-arrange to compensate for a changing interface. In the case of adsorption reflected from the surface tension behavior, I conclude that NIPAM polymers should first diffuse to the air/water interface, then polymer chains more or less unwrap and spread out to cover the air/water interface. It is proposed that less crosslinked NIPAM polymers occupy the air water interface more efficiently because of higher polymer chain mobility. At the air/water interface, it is inferred that $N$-isopropyl groups tend to protrude into the air phase at all the temperatures. 


\subsection{Contribution}

The main contributions of this thesis to science and potential applications of NIPAM polymers are:

1. For the first time, the interfacial properties of linear NIPAM homopolymers, copolymers, crosslinked NIPAM microgels and macrogels are studied and compared.

2. For the first time, it is experimentally demonstrated that crosslinked colloidal microgels lower the surface tension of water and attempt is made to model the dynamic surface tension through a one parameter Langmuir kinetics model.

3. For the first time, an ESEM picture showing microgels adsorbed at the air/water interface is ever reported.

4. For the first time, it is found that phase-separated colloidally stable particles of linear NIPAM polymers lower the surface tension of water.

5. A description of PNIPAM at the air/water interface after the phase separation in the bulk phase is first proposed, which will be refined based on the results from neutron reflectivity experiments in progress.

6. Fundamentally, it is discovered that adsorbed PNIPAM at the air/water interface does not phase separate below $40^{\circ} \mathrm{C}$, whereas it has an LCST of about $32{ }^{\circ} \mathrm{C}$ in an aqueous bulk phase. 
7. For the first time, surface tension kinetics have been modeled using both experimental and theoretical models of the relationship between surface tension $(\gamma)$ and adsorbed polymer concentration (T).

8. The application of the Langmuir kinetics model and van Eijk and Cohen Stuart's spreading disk model to predict the dynamic surface tension of NIPAM homopolymer and comparison of their prediction power are unique.

9. For the first time, the dynamic surface tension of an oscillating air/water interface is mathematically described in a non-spreading disc model.

10. For the first time, the surface tension lowering ability of poly(NIPAM-coAM) copolymers is experimentally investigated and predicted through a novel one-parameter model derived from a model of the excess free energy of mixing.

11. For the first time, it is reported that advancing contact angles of water on water-swollen gels are temperature sensitive and not zero. 